式

Dynamic Sitting

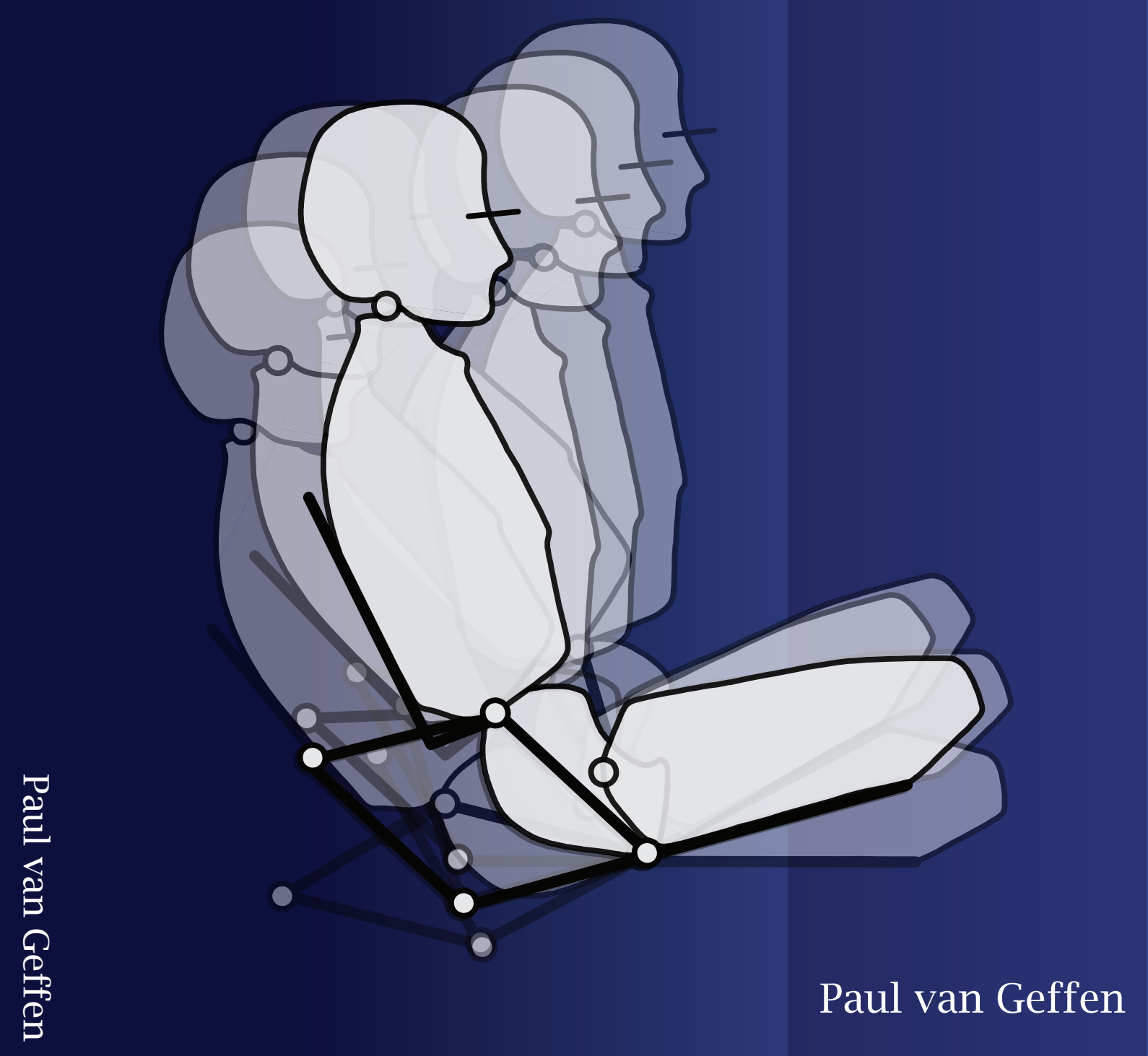




\section{Dynamic Sitting}


This work was supported by SenterNovem (grant TSIT3043) and the Institute for Biomedical Technology, Enschede.

\section{SenterNovem}

\section{BMT के}

University of Twente

Enschede - The Netherlands

The publication of this thesis was financially supported by the following companies. Their support is gratefully acknowledged.

Xsens Technologies BV, Enschede, The Netherlands (see page 208)

Positioning Products BV, Den Haag, The Netherlands (see page 208)

PR-Sella BV, Oldenzaal, The Netherlands

De promotiecommissie is als volgt samengesteld:

Voorzitter en Secretaris:

Prof.dr. F. Eising

Universiteit Twente

Promotoren:

Prof.dr.ir. H.F.J.M. Koopman Universiteit Twente

Prof.dr.ir. P.H. Veltink

Universiteit Twente

\section{Leden:}

Prof.dr.ir. J.B. Jonker

Universiteit Twente

Prof.dr.ir. J. van Amerongen.

Universiteit Twente

Prof.dr. H.J. van Dieën

Vrije Universiteit Amsterdam

Prof.dr. J.S. Rietman

Universiteit Twente

Prof.dr. L.H.V. van der Woude

UMC Groningen

Dr.ir. C.W.J. Oomens

Technische Universiteit Eindhoven

\section{Paranimfen:}

Jasper Reenalda

Jos van Geffen

English title: Dynamic Sitting

Nederlandse titel: Dynamisch Zitten

Printed by: Ipskamp Drukkers BV, Enschede, The Netherlands

ISBN: $\quad 978-90-365-2840-5$

Copyright (c) 2009 by P. van Geffen, Amsterdam, The Netherlands.

All rights reserved. No parts of this publication may be produced or transmitted in any form or by any means, electronic or mechanical, including photocopy, recording or any information storage or retrieval system, without prior written permission of the holder of the copyright. 


\title{
Dynamic SitTing
}

\author{
PROEFSCHRIFT
}

ter verkrijging van

de graad van doctor aan de Universiteit Twente, op gezag van de rector magnificus,

prof.dr. H. Brinksma, volgens besluit van het College voor Promoties in het openbaar te verdedigen op donderdag 14 mei 2009, om 13:15 uur

door

\section{Paul van Geffen}

Geboren op 9 mei 1978

te Waarland 
Dit proefschrift is goedgekeurd door promotoren:

Prof.dr.ir. H.F.J.M. Koopman

Prof.dr.ir. P.H. Veltink

ISBN 978-90-365-2840-5

(C)2009 P. van Geffen 


\section{Contents}

$\underline{\text { I General Introduction }} \underline{7}$

Chapter 1 General Introduction 9

II Postural Control $\quad \underline{21}$

Chapter 2 Pelvis Control from Seat Support Forces 23

Chapter 3 Body Segments Decoupling to Control Sitting Posture 35

III Regulation of Body Load $\quad \underline{49}$

Chapter $4 \quad$ Sagittal Chair Adjustment and Seat Reaction Load 51

Chapter 5 Decoupled Pelvis Rotations to Regulate Buttock Load 65

Chapter 6 Dynamic Tuberal Support to Regulate Ischial Buttock Load 79

Chapter 7 Decoupled Pelvis Rotations to Regulate Low Back Load 97

IV Clinical Evaluation $\quad \underline{113}$

Chapter 8 Seating Interventions that Regulate Body Load in Spinal Cord 115 Injured Individuals

Chapter 9 Decoupled Pelvis Alignment and Functional Movement in

Spinal Cord Injured Individuals

V General Discussion $\quad \underline{145}$

$\begin{array}{lll}\text { Chapter } 10 \quad \text { General Discussion and Conclusions } & 147\end{array}$

$\begin{array}{ll}\text { References } & 161\end{array}$

Appendix A Design Process 173

$\begin{array}{lll}\text { Appendix B } & \text { Research Consortium } & 179\end{array}$

$\begin{array}{lll}\text { Appendix C List of Abbreviations } & 183\end{array}$

$\begin{array}{ll}\text { Summary } & 187\end{array}$

Samenvatting 193

Dankwoord 199

Curriculum Vitae 205 


\section{Part I}

General Introduction 


\section{Chapter 1}

\section{General Introduction}




\subsection{Introduction}

An impaired neuromuscular function of the trunk and lower extremities makes that many wheelchair-users suffer postural instability and have problems with the performance of functional movement during activities of daily life (Chen et al., 2003, Chaffin et al., 2004, Curtis et al., 1995, Lanzetta et al., 2004, Seelen et al., 2001, Seelen et al., 1997). Functional balance during unsupported movement of the trunk and upper extremities relies on adequate neuromuscular proprioceptive responses of the central nervous system that keeps the body centre of mass within specific stability limits.

To increase the base of support and to bring the body centre of mass closer to the support surface, wheelchair-users who lack postural control often adopt a static kyphotic spinal posture with posterior tilted pelvis (Hobson and Tooms, 1992, Koo et al., 1996). Some individuals even recline the seat support and squeeze the pelvis and lower spine against the backrest for additional postural stability during upperbody movement tasks (Maurer and Sprigle, 2004). Such seating conditions allow much internal and external perturbation before reaching the boundaries of postural instability which makes that very little postural control is needed to remain posturally stable (Seelen et al., 1997). At the same time, very much effort is needed to change body posture for functional purpose and therefore a static body posture is often adopted. Because the motion of the spine is constrained between the posterior tilted pelvis and the backrest, the ability for repositioning is negligible and the performance of functional movement is limited to movement of the upper extremities only (Faiks and Reinecke, 1998).

In many wheelchair-users, prolonged static sitting leads unconditionally to all kinds of physical discomfort including the formation of pressure ulcers (Collins, 1999), low back injury (Lengsfeld et al., 2000, Makhsous et al., 2003, van Deursen et al., 2000, Ferguson and Marras, 1997, Samuelsson et al., 1996), lumbar immobility and joint stiffness (Beach et al., 2005). It has been accepted with some certainty that most problems occur from sustained mechanical tissue loading and that dynamic seating interventions are needed when individuals cannot physically reposition themselves (Crane et al., 2007). The inability to change body posture underlines the importance for automatic chair modification or the help of others. Because this latter often involves a time consuming and labour intensive activity, we strive to prevent mobility problems by the use of dynamic systems that adjust sitting posture and relieve body structures periodically.

\subsection{Target Group}

The main target group of this research involves all wheelchair-users who suffer mobility problems due to prolonged static sitting. Because physical discomfort is also a concern in able-bodied persons who do sedentary work (i.e. office-workers and car drivers) and sit still in confined setting for hours, this group also receives attention. However, we mainly focus on individuals with a spinal cord injury (SCI) because of the devastating impact on the patient's functional, medical, financial and psychosocial well-being and its significant costs to society (DeVivo, 1997, Ackery et al., 2004, Post et al., 1998). 


\subsubsection{Spinal cord injury}

A SCI is an insult to the spinal cord and often involves major neurological consequences to motor, sensory and autonomic functioning of body structures below the level of lesion (American Spinal Injury Association, 2000).

As a rough estimation, worldwide prevalence of individuals with a SCI has been reported around 223-755 per million inhabitants. Incidence rate lies between 10.4 and 83 per million inhabitants per year and mostly involves young male adults (80\% male, 20\% female) in all layers of society (Wyndaele and Wyndaele, 2006). Although this relatively small incidence rate reflects that a SCI is a fairly uncommon disability, its social and economic costs are far out of proportion (DeVivo, 1997, Post et al., 1998).

SCI due to a trauma is most common and is mainly caused by motor vehicle accidents (45.6\%), falls (19.6\%), violence (17.8\%) and sports injuries (10.7\%) (Jackson et al., 2004). Published by the American Spinal Injury Association (ASIA), the International Standards for Neurological and Functional Classification of Spinal Cord Injury involves a widely accepted classification tool for clinicians in the assessment and care of SCI-individuals (American Spinal Injury Association, 2000). It describes the level of lesion and the extent of injury based on a systematic motor and sensory examination of neurological functioning.

As a rough distinction between injuries, a lesion in the cervical region with function loss in the trunk and all four extremities is classified as tetraplegia, and a lesion in the thoracic, lumbar, or sacral region with function loss in the lower extremities is classified as paraplegia. Further differentiation can be made by the ASIA Impairment Scale (AIS).

When no sensory or motor function is preserved below the level of injury, the extent of injury is classified as complete, and when either (some) motor or sensory is preserved, the extent of injury is classified as incomplete. Although the terminology for tetraplegia and paraplegia has become obsolete, it is still used as a first indication about the neurological level of injury.

Based on these standards for neurological and functional classification, Jackson et al. (Jackson et al., 2004) reported that 30.6\% of all SCI can be classified as incomplete tetraplegia, $26.1 \%$ as complete paraplegia, $23.4 .5 \%$ as complete tetraplegia, and $19.2 \%$ as incomplete paraplegia. A lesion around the fifth cervical vertebrae (C5) is most common in tetraplegic individuals, and around the twelfth thoracic vertebrae (Th12) in paraplegic individuals. Although the classification and assessment of a SCI seems rather straightforward, in practice diversity in muscle functioning for lesions with similar classification are remarkable.

In addition to the potential loss of sensation and motor function below the level of injury, many individuals with a SCI also suffer secondary complications (Ackery et al., 2004). Urologic, gastro-intestinal and genital disorders, spasticity, pain, blood circulation and temperature disturbance, respiratory dysfunction and psychological problems are all factors that can enormously affect someone's well-being and are therefore important to take into account.

In this thesis, we mainly focus on the limited ability for functional movement and the development of physical discomfort that occur from prolonged static sitting. Regarding the functional independence of wheelchair-users, we strive to design 
seating interventions that provide postural stability without constraining functional movement, and prevent physical discomfort (i.e. pressure ulcers, lumbar immobility and low back pain) in prolonged static sitting.

\subsection{Mobility Problems}

\subsubsection{Reduced postural stability}

To increase the base of support and to bring the body centre of mass closer to the support surface, individuals who lack postural control often adopt a kyphotic spine posture with posterior tilted pelvis (Hobson and Tooms, 1992, Koo et al., 1996). Some individuals even recline the seat support and squeeze the pelvis and lower spine against the backrest for extra postural stability during upper-extremities movement tasks (Maurer and Sprigle, 2004). Clinical observations have indicated that many impaired individuals 'feel' more stable when sitting with posterior tilted pelvis (Maurer and Sprigle, 2004). However, such flexed spine postures have negative influence on body movement in forward direction (Faiks and Reinecke, 1998). Because the motion of the spine is constrained between the posterior tilted pelvis and the backrest, the ability to reach forward is limited to the shoulder girdle only.

Regarding the functional independence of wheelchair-users, important criteria for new wheelchair design involve interventions that provide postural stability without constraining functional movement. The ability to balance the trunk during unsupported body movement has therefore often been analysed (Janssen-Potten et al., 2001, Janssen-Potten et al., 2000, Janssen-Potten et al., 2002, Curtis et al., 1995). In their search to find a trade-off between postural stability and freedom of trunk and upper extremity movement, Janssen-Potten et al. evaluated sitting balance in spinal cord injured (SCI) individuals for different chair modifications (Janssen-Potten et al., 2000, Janssen-Potten et al., 2001). They compared the effects of forward seat inclination, chair recline (tilt-in-space) and backrest recline with a standard chair configuration, but found no influence on postural control in high (Th2-Th8) SCIindividuals.

Because the pelvis forms the basis for trunk support and directly affects the curvature of the lumbar spine, it has been recognized that the ability to control pelvis alignment in anterioposterior direction plays an important role in the control of body posture, trunk and upper extremity movement. Seating interventions that adjust pelvis angle in sagittal direction might therefore be applicable to regulate spinal posture and to improve the functional performance in daily wheelchair-use.

\subsubsection{Lumbar immobility and low back discomfort}

It has been reported that the lumbar spine characteristics change in prolonged sitting with flexed lumbar spine curvature (Beach et al., 2005, Parkinson et al., 2004). Within the first two hours of sitting, Beach et al. (Beach et al., 2005) found an increase in the passive flexion stiffness in moderate ranges of lumbar flexion. This demonstrates that spinal tissue characteristics already change after relatively short periods of exposure. They associated this stiffness increase with an increased passive resistance of muscles which they assumed being the primary flexion-resisting tissues. 
Beach et al. (Beach et al., 2005) and Parkinson et al. (Parkinson et al., 2004) reported that these findings suggest an increased risk for low-back injury when individuals perform full lumbar flexion tasks after prolonged sitting with flexed lumbar posture. When flexed lumbar spine postures are maintained, continuous stressing of the lower back will eventually cause the stiffness of the posterior spine ligaments and intervertebral discs to decrease due to visco-elastic creep (Keller et al., 1987, Argoubi and Shirazi-Adl, 1996, Hedman and Fernie, 1997).

Sustained mechanical spinal loading without significant durations of stress relief causes nutrient supply to stagnate and load bearing spinal structures to degenerate (Urban et al., 2004). Because the intervertebral discs and facet joints are reported to be important structures to maintain segmental stability, degenerative processes in these structures change the segment motion characteristics associated with spinal instability and low back injury (Fujiwara et al., 2000a, Fujiwara et al., 2000b). A lordotic lumbar spine curvature together with significant levels of spinal movement are needed to facilitate the perfusion of intervertebral disc nutrition (Holm and Nachemson, 1983) and to decrease the risk for disc degeneration (Urban et al., 2004).

To reduce incidence of low back injury in individuals that undergo insufficient postural variation, several techniques have been reported that enhance lumbar motion and relieve static loads associated with spinal tissue degeneration and pain (van Deursen et al., 1999, Aota et al., 2007, Reinecke et al., 1994). Van Deursen et al. (van Deursen et al., 1999) described an ergonomic chair that uses passive motion for continuous axial pelvis rotation imposed by extremely small rotational seat movements in the horizontal plane. Based on subjective measures for lumbar discomfort, they reported a positive effect on pain relief in subjects suffering low back injury. Although they never measured the mechanical or physiological effects on the internal spine conditions, they explained this reduced sensation of discomfort to improved processes of osmosis and diffusion for nutrient to the intervertebral discs.

To find proof for the efficacy on pain relief, Lengsfeld et al. (Lengsfeld et al., 2007) subjected this ergonomic chair to a rigorously designed randomized multicenter study under 280 office-workers suffering low back pain. After two years of observations, no significant difference was found when comparing the effects on lumbar comfort for the ergonomic chair and other high quality office-chairs. They attributed this lack of significant results to the minimal degree of axial rotation amplitude and they suggested that considerable higher rotation amplitudes might provide better outcomes.

Contrary to the concept of induced axial spinal rotations, some techniques have been reported that affect spine curvature in sagittal direction. Reinecke et al. (Reinecke et al., 1994) described a commercially available device (Backcycler ${ }^{\circledR}$, ergonomics Inc., Winooski, VT) that induces continuous passive motions to the lower spine by an alternating inflatable lumbar support. Based on subjective measures for low back discomfort, they reported an improved tolerance for prolonged sitting.

Beach et al. (Beach et al., 2003) and Atoa et al. (Aota et al., 2007) also investigated the effects of this adjustable lumbar support in prolonged sitting. Beach et al. found no differences in activation patterns of the lower erector spinae muscle, and suggested that the potential benefits for pain relief might be caused by the facilitation of the 
intervertebral disc nutrient. Atoa et al. did also not observed any statistical evidence for a reduced feeling of lumbar discomfort. However, they reported that the induced lumbar motion was less than what was found by Reinecke et al. (Reinecke et al., 1994), which might explain why no positive effect was found. Adjustment of the lumbar support that induces a more profound effect on lumbar motion might increase the beneficial effect on lumbar pain relief in sitting.

\subsubsection{Pressure ulcers}

The inability to change body posture causes continuous buttock loading, which potentially initiates the irreversible process for pressure ulcers in the tissue under the sacrum and ischial tuberosities (Bouten et al., 2003).

Graded by the classification system of the European Pressure Ulcer Advisory Panel (EPUAP), the severity of tissue injury varies from superficial skin irritation to extensive destruction/necrosis of deeper tissue layers with or without loss of skin thickness (Black et al., 2007, Defloor and Schoonhoven, 2004, Defloor et al., 2005). It has been reported (Bouten et al., 2003) that superficial injury develops in the skin mainly from friction and shear at the buttock-seat interface, and that deep injury develops in the muscle tissue under the bony prominences from sustained deformation and impaired tissue perfusion. This latter form of tissue injury is a severe pressure ulcer of which the onset is hard to detect since it initiates in deeper muscle layers. It progresses outwards to the skin and when it manifests at the buttock surface it already caused severe damage to the sub-dermal tissue. This type of pressure ulcer is extremely hard to treat and sometimes even results in surgery with serious consequences for someone's well-being.

Extensive studies have been performed to determine the cause, onset, location and extent of tissue damage from sustained internal tissue loading. Evidence from experimental research with muscle-cell cultures (Gawlitta et al., 2007, Gefen et al., 2008a, Gawlitta et al., 2008, Gefen et al., 2008b), finite element models (LinderGanz et al., 2007, Brosh and Arcan, 2000, Ragan et al., 2002, Todd and Thacker, 1994, Lim et al., 2007, Makhsous et al., 2007a), animals (Linder-Ganz et al., 2006, Stekelenburg et al., 2006) and humans (Ohura et al., 2007, Quintavalle et al., 2006) have demonstrated that deep tissue injury initiates subdermally and that its primary cause of onset involves more than localised tissue ischemia only.

Recent investigation of Stekelenburg at al. showed that a complex interplay with prolonged excessive deformation potentially catalyses the irreversible process of tissue breakdown (Stekelenburg et al., 2008, Stekelenburg et al., 2007). Although the exact cause for pressure ulcers formation has not completely been revealed, it has been accepted that periodic pressure relief is needed to recover the buttock tissue from continuous deformation and impairment of tissue perfusion.

To reduce incidence of pressure ulcers, common applications are based on seating surfaces that distribute the buttock pressure over a larger area and decrease peak pressures under the ischial region. Although the overall buttock pressure will reduce significantly, it is unlikely that this alleviates the ischial buttock tissue enough to maintain tissue perfusion and fully prevent pressure ulcer onset (Reswick and Rogers, 1976).

For periodic pressure relief, wheelchair-users are often recommended to shift 
body posture functionally. Leaning forward and pushing up from the armrests are considered effective movement strategies and are therefore part of many repositioning programs that prescribe wheelchair-users to undertake body movement every 15 minutes (Agency for Health Care Policy and Research (AHCPR), 1994). Ischial peak pressures relieve up to $40 \%$ when leaning forward $45^{\circ}$ and even up to $80 \%$ when leaning forward completely (Henderson et al., 1994, Koo et al., 1996). For full recovery of localised tissue oxygenation and perfusion, there is evidence that for some individuals the buttock must be unloaded for at least two minutes (Coggrave and Rose, 2003, Makhsous et al., 2007b, Bader, 1990). This requires good upper-body strength and is therefore not feasible for many impaired wheelchair-users.

When individuals cannot reposition body posture functionally, automatic chair recline (tilt-in-space) is an effective alternative for periodic pressure relief. It is reported that the ischial peak pressures relieve 32\%, 40\% and 47\% when reclining the chair $35^{\circ}, 50^{\circ}$ and $65^{\circ}$ respectively (Henderson et al., 1994, Sprigle et al., 2007). Although the benefits of body movement on pressure relief have been demonstrated, many wheelchair-users do not concord with prescribed movement programs or other clinical repositioning advice, even when they posses adequate cognitive and physical ability to relieve buttock pressure from either functional repositioning or automatic chair modification (Stockton and Parker, 2002).

When individuals cannot reposition themselves and static seating surfaces do not relieve buttock pressure sufficiently, dynamic systems that adjusts the seating surface are useful within proper pressure management (Makhsous et al., 2007c, Stockton and Rithalia, 2007). However, it must never be the intention to apply dynamic seating surfaces and completely replace the need for postural movement, especially when prolonged static sitting is also associated with mobility problems other than pressure ulcers. With this in mind, we strive to redistribute buttock load and to maintain tissue perfusion from local seat support manipulations together with global chair modification and enhancement of functional movement.

\subsection{Research Goals}

In this research we develop an experimental simulator chair and we evaluate several seating interventions that are designed to enhance functional movement and to prevent physical discomfort due to prolonged static sitting. Theoretical and experimental evaluation will be performed in able-bodied subjects and in subjects with a spinal cord injury (SCI) to answer the following research questions:

Q1 How can sitting posture be controlled?

Q2 What interventions are effective to regulate body load associated with physical discomfort (i.e. pressure ulcers and low back pain)?

Q3 What interventions benefit the performance of functional movement in impaired sitting? 


\subsection{Research Approach}

When someone cannot reposition him-/herself physically, adequate postural variation can only be realised from automatic chair modification or by the help of others. Because the latter often involves a time consuming and labour intensive activity, we try to enhance the ability for repositioning by adjusting the global configuration of the chair. To benefit the functional independence of the user, we discriminate between three principles to regulated body load in daily wheelchair sitting:

1. Physical repositioning, which refers to the users' physical ability to reposition body posture functionally. Chair configurations that involve a trade-off between posture stability and freedom of upper body movement might enhance the preserved ability to carry out some functional movement strategies without losing balance.

2. Passive repositioning, which refers to global chair modifications that adjust body posture in a passive manner. This means that the individual remains passive and undergoes postural variation as imposed by the chair.

3. Local support surface manipulation, which refers to local adjustment of body supports (e.g. seat support and backrest) for periodic manipulation of body structures without affecting sitting posture.

Able-bodied individuals do not suffer physical discomfort since they continuously shift body posture physically and thereby regulate body load in a functional manner. In individuals with reduced or no postural stability, the lack of functional body movement must be compensated by automatic chair modifications that control body posture and manipulate the support surface locally. The less upper body function is preserved, the more periodic surface manipulation is needed to alleviate load bearing structures and consistently prevent that degeneration of body tissue takes place. The latter mainly counts for the load bearing tissue of the ischial buttock region. Because the soft tissue in this area is most vulnerable for sustained mechanical deformation, adjustable support elements must be integrated in the seating surface. We expect that periodic manipulation of the support surface in this region will help the ischial buttock tissue to recover from continuous deformation and impairment of tissue perfusion in prolonged static sitting. With this in mind, a fully adjustable and computer-aided simulator chair has been developed.

\subsubsection{Computer-aided simulator chair}

The most important functional requirements involve the possibility to control body posture, enhance functional movement, and to regulate body load associated with pressure ulcers and low back pain. Based on these requirements a simulator chair was built as shown in figure 1.1.

Based on a parallelogram design that aligns the chair pivots with the anatomical axes for body segments rotation, an intervention was implemented that controls the alignment of the trunk, pelvis and thighs separately. Furthermore, two adjustable 


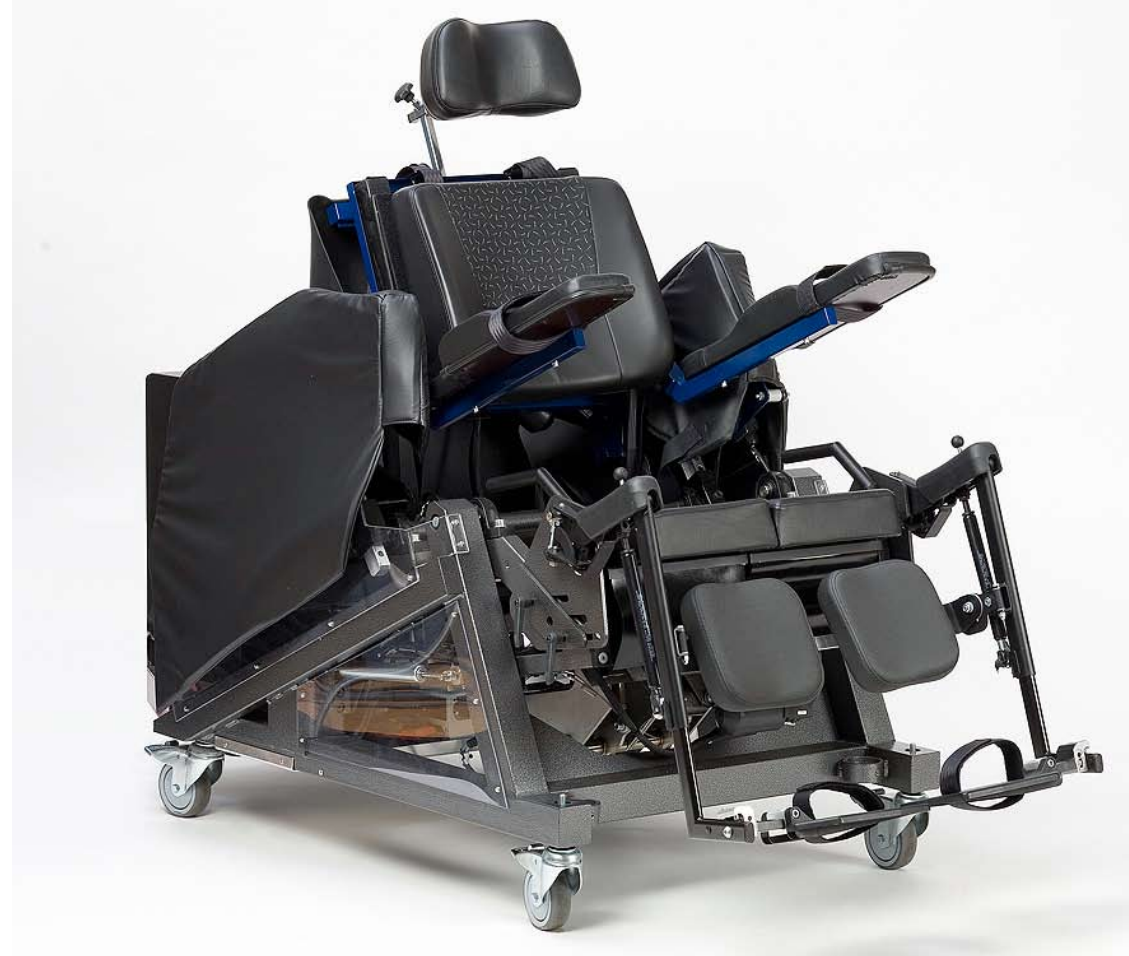

Figure 1.1: A prototype of the adjustable computer-aided simulator chair. In close collaboration with our group, the simulator chair was designed and built by the engineering company Demcon BV (Oldenzaal, the Netherlands).

support elements were integrated under the ischial tuberosities for additional manipulation of the ischial buttock tissue. For a more detailed description about the design of the simulator chair and its integrated features, we refer to appendix A.

The simulator chair is involved in most of the experiments. We investigate whether the integrated techniques are effective to control body posture, enhance functional movement and to alleviate the load bearing tissue of the buttock and lower back. Based on experiments in which we subject able-bodied and SCI-individuals to alternating protocols of posture adjustment and local support surface manipulation, we try to answer our initial research questions as stated in paragraph 1.4.

It is important to notice that all experiments in this thesis were approved by the local Committee for Medical Ethics of the Roessingh Rehabilitation Centre (Enschede, the Netherlands) and all participating subjects gave their written informed consent about the objectives and experimental protocol. 


\subsection{Thesis Outline}

\section{General Introduction}

The first section of this chapter underlined the importance of dynamic seating interventions to control body posture and to prevent physical discomfort in persons who lack the strength to reposition sitting posture physically. Therefore, we develop an experimental simulator chair and we evaluate several seating interventions that are designed to enhance functional movement and to prevent physical discomfort due to prolonged static sitting.

\section{Postural Control}

The alignment of the pelvis plays a key role in the assessment of body posture and wheelchair configuration in relation to wheelchair seating discomfort. Because pelvis angle directly affects spinal curvature, postural stability and the forces that exert on the lower back and under the ischial buttock region, control of the pelvis is important. To monitor pelvis angle for proper pelvis control, chapter 2 explores whether it is feasible to predict pelvis angle from seat support forces based on a theoretical analysis and measurements for experimental validation. Proper control of body posture also involves the ability to move the trunk and thighs separately. We therefore instrumented the simulator chair with an intervention that decouples the trunk, pelvis and thighs by aligning the chair pivots with the anatomical axes for body segments rotation. Based on experimental measurement of body posture and chair segments modification in healthy individuals, chapter 3 develops a predictive model that computes angular chair configuration for desired body postures.

\section{Regulation of Body Load}

When we know how angular chair adjustment influence sitting posture, the next step is to evaluate whether the simulator chair is also applicable to regulate body load. These research questions are dealt with in chapters 4, 5, 6, 7 and 8. Chapter 4 focuses on the effects of chair modification on buttock load in the sagittal plane. In addition, chapter 5 investigates the effects of chair modification in sagittal and frontal direction, and looks at the alignment of the pelvis rather than chair configuration only. Where chapter 4 and 5 focus to the effects of global chair modifications on buttock load, chapter 6 describes a system for local seat support manipulation and evaluates how this effects the distribution of buttock load and blood supply to the skin and subcutaneous tissue under the ischial tuberosities. Similar to chapter 5, the same protocol for global chair modification is repeated in chapter 7 . However, this chapter investigates the effects on the forces that exert at the lower back and discusses the potential benefits for low back injury in prolonged sitting with a static spinal posture. It is the only chapter which does not directly focus on wheelchairusers. Because it has been written from an industrial perspective, we aim at car drivers and office-workers instead.

For clinical practice, the effects of local and global chair adjustment on body load 
must also be evaluated in individuals who cannot functionally reposition themselves. Impairments to the neuromuscular function influence postural response (e.g. muscle spasms) from chair modification and make clinical investigation necessary. Chapter $\boldsymbol{8}$ therefore investigates the effects of local support surface manipulations and global chair modification on the forces at the buttock-seat interface and lower back in SCI-individuals. Besides the potential benefits for body load, important criteria for new wheelchair design also involve interventions that provide posture stability without constraining functional movement. The ability to balance the trunk during unsupported body movement has therefore been analysed in chapter 9.

\section{General Discussion and Conclusions}

At the end of this thesis, chapter 10 looks back to our main objectives and gives answer to the initial research questions. It provides a general discussion and evaluates future prospects that are necessary to bring our knowledge into clinical practice.

\section{References}

This section lists all references in alphabetic order.

\section{Appendices}

Three appendices are included after the reference section. Appendix A gives a short description about of the design process. Appendix $\boldsymbol{B}$ introduces the partners of the consortium, and explains their contribution in the project. For the convenience of the reader, a list of frequently used abbreviations is included in appendix $\boldsymbol{C}$. We expect that this facilitates the reading process and improves the interpretation of our findings.

\subsection{Additional remarks}

As the reader will notice, most chapters are written as journal articles and do not always appear in chronological order. The order of appearance is based on how it fits best in the context of our research. Some chapters have already been published. The text of these publications has mostly been kept intact. This makes that each chapter can be read separately. As a consequence, parts of the introduction and method sections seem repetitive. However, each chapter has a different accent and focuses on other aspects of our research. 


\section{$\underline{\text { Part II }}$}

\section{Postural Control}




\section{Chapter 2}

\section{Pelvis Control from Seat Support Forces}

Published as: P. van Geffen, P.H. Veltink and H.F.J.M. Koopman, 2009, Can pelvis angle be monitored from seat support forces in healthy subjects? Journal of Biomechanical Engineering, Volume 131, Issue 3, 034502. 


\begin{abstract}
Individuals who cannot functionally reposition themselves often need dynamic seating interventions that change body posture from automatic chair adjustments. Pelvis alignment directly affects sitting posture, and systems that adjust and monitor pelvis angle simultaneously might be applicable to control body posture in sitting. The present study explores whether it is feasible to monitor pelvis angle from seat support forces. Pelvis angle estimation was based on equivalent 'two-force member' loading for which pelvis orientation equals the orientation of the equivalent contact force. Theoretical evaluation was done to derive important conditions for practical application. An instrumented wheelchair was developed for experimental validation in healthy subjects. Seat support forces were measured and mechanical analysis was done to derive the equivalent contact force from which we estimated pelvis angle.

Model analysis showed a significant influence of pelvis mass, hip force and lumbar torque on the relation between the actual pelvis angle and the predicted pelvis angle. Proper force compensation and minimal lumbar torque seemed important for accurate pelvis angle estimations. Experimental evaluation showed no body postures that involved a clear relation between pelvis angle and the orientation of the equivalent contact force.

Findings suggest that pelvis angle could not be estimated in healthy individuals under the described experimental seating conditions. Validation experiments with impaired individuals must be performed under different seating conditions to provide a better understanding whether the principle is of interest for clinical application.
\end{abstract}




\subsection{Introduction}

Wheelchair users who cannot functionally reposition themselves often adopt a static body posture (Alm et al., 2003), and suffer from sitting related mobility problems such as pressure ulcers (Collins, 1999), low back injury (Ferguson and Marras, 1997, Hedman and Fernie, 1997), respiratory dysfunction (Lin et al., 2006), lumbar immobility and joint stiffness (Beach et al., 2005, Parkinson et al., 2004) in long-term sitting. The inability to reposition underlines the importance for dynamic seating interventions that control body posture from automatic chair adjustments. Because pelvis alignment directly affects sitting posture (Hastings et al., 2003, Janssen-Potten et al., 2001, Delisle et al., 1997, Sprigle et al., 2002), systems that adjust and monitor pelvis angle simultaneously might be applicable for proper posture control.

In a previous study (van Geffen et al., 2008), we described a seating system that effectively adjusts the pelvis independent from the trunk and seat support. For proper pelvis control however, continuous feedback from pelvis angle is essential. Several non-invasive measuring techniques (X-rays, goniometers, 3D skin marker reflecting methods, etc.) have been used to monitor pelvis angle in sitting (Sprigle et al., 2003, Sprigle et al., 2002, Lalonde et al., 2003, Lazennec et al., 2004, Moes, 1998, Campbell et al., 2001, Lucas et al., 2005). Skin artefacts (Lalonde et al., 2003) and extensive lab setups make real-time feedback difficult, and an alternative is therefore needed. Because the pelvis is supported by the seat, close relations are expected between pelvis position and seating forces.

To monitor pelvis alignment for proper pelvis control, the present study explores whether it is feasible to predict pelvis angle from seat support forces based on a theoretical analysis and measurements for experimental validation.

\subsection{Methods}

\subsubsection{Approach}

The estimation of pelvis angle is based on equivalent 'two-force member' loading (Meriam and Kraige, 1993) in which pelvis orientation equals the orientation of the equivalent contact force. Figure 2.1A shows a schematic representation of an adopted sitting posture in the sagittal plane. The trunk is supported above the pelvis, the arms by the armrests, the pelvis and thighs by the seat, and the lower legs by the leg rests. Support of the trunk above the lumbosacral spine makes the pelvis function as the foundation for trunk support guiding the forces of the upper body $\left(F_{1}\right)$ to the seat. Other forces that exert on the pelvis are the tuberal contact force $\left(\mathrm{F}_{t}\right)$, pelvis gravitational force $\left(G_{p}\right)$, hip force $\left(F_{h}\right)$ and lumbar torque $\left(T_{1}\right)$. Hip torque is neglected. As shown in figure $2.1 \mathrm{~B}$, the equivalent contact forces $\left(\mathrm{F}_{\text {eq, }}\right.$ and $\left.\mathrm{F}_{\text {eq, }}\right)$ substitute all forces $\left(F_{1}, F_{h}, F_{t}\right.$ and $\left.G_{p}\right)$ that exert on the pelvis. The orientation of $F_{e q, t}\left(\psi_{e q}\right)$ equals $\alpha$ in the absence of lumbar torque. Equations 1-5 show how to derive $\mathrm{F}_{\text {eq,t }}$ and $\mathrm{F}_{\text {eq, } \mathrm{l}}$

$$
\begin{aligned}
& \bar{F}_{e q, l}=\bar{F}_{l}+f_{h} \cdot \bar{F}_{h}+f_{p} \cdot \bar{G}_{p} \\
& \bar{F}_{e q, t}=\bar{F}_{t}+\left(1-f_{h}\right) \cdot \bar{F}_{h}+\left(1-f_{p}\right) \cdot \bar{G}_{p}
\end{aligned}
$$




$$
\begin{aligned}
& \bar{F}_{e q, t}=-\bar{F}_{e q, l} \\
& \bar{T}_{l}=\bar{F}_{e q, t} \cdot d
\end{aligned}
$$

in which,

$f_{h}=\frac{d_{h}}{l_{p}}, \quad f_{p}=\frac{d_{p}}{l_{p}}, \quad d=l_{p} \cdot \sin \left(\alpha-\psi_{e q}\right)$

\subsubsection{Model simulations}

Theoretical evaluation to predict pelvis angle from seat support forces was performed using a simplistic rigid body model of a seated person. Model simulations predicted its feasibility and derived important conditions for practical application.

Positive body segment angles $(\alpha, \beta$ and $\gamma$ ), body dimensions and body forces are shown in figure 2.2. Pelvis angle $(\alpha)$ and trunk angle ( $\beta$ ) are expressed relative to the vertical. Thigh angle $(\gamma)$ is expressed relative to the horizontal. The arms and lower legs are supported that no joint forces acted in the shoulders and knees. All body parts are modelled as rigid bodies and connected with hinge joints each having one degree of freedom. Centre of masses lie on the line connecting two adjacent joints. Head orientation was kept vertical (Campbell et al., 2001, Pozzo et al., 1995) and shear forces on the trunk and thighs were neglected. The axis of pelvis rotation lies under the ischial tuberosities and was modelled as a pivot with one degree of freedom.

Based on studies to flexion-extension mechanics of the lumbar spine (Dickey and Gillespie, 2003, Gillespie and Dickey, 2004), a passive joint stiffness was introduced between the pelvis and trunk. Analysis was done for an average male subject. Body parameters (Staarink, 1995) are defined in table 2.1. Static equations of equilibrium for individual body segments were derived and the seat support forces were computed. During simulation, pelvis angle $(\alpha)$ changed over a range of $40^{\circ}\left(15^{\circ}<\alpha<55^{\circ}\right)$ relative to a fixed trunk $\left(\beta=24^{\circ}\right)$ and different thigh angles $\left(\gamma_{1-4}=0^{\circ}, 12^{\circ}, 24^{\circ}\right.$ and $\left.36^{\circ}\right)$. Thigh angle $(\gamma)$ affected hip force $\left(\mathrm{F}_{\mathrm{h}}\right)$ and was altered from adjusting seat inclination. Orientations of $F_{t}(\psi)$ and $F_{\text {eq, }}\left(\psi_{\text {eq }}\right)$ were computed and related to $\alpha$. Furthermore, the individual influence of hip force $\left(\mathrm{F}_{\mathrm{h}}\right)$, pelvis mass $\left(\mathrm{G}_{\mathrm{p}}\right)$ and lumbar torque $\left(\mathrm{T}_{1}\right)$ on pelvis angle estimation $(\hat{\alpha})$ was derived from orientations of $F_{1}\left(\psi_{1}\right), F_{2}\left(\psi_{2}\right)$ and $F_{\text {eq, }}\left(\psi_{\text {eq }}\right)$ respectively, as shown in figure 2.5. Lumbar torque is absent for $\mathrm{F}_{1}$ and $\mathrm{F}_{2}$. Equations 6 and 7 show how to derive $F_{1}$ and $F_{2}$ respectively.

$$
\begin{aligned}
& \bar{F}_{1}=\bar{F}_{t}+\left(1-f_{p}\right) \cdot \bar{G}_{p} \text { with } \bar{T}_{l}=0 \\
& \bar{F}_{2}=\bar{F}_{t}+\left(1-f_{h}\right) \cdot \bar{F}_{h} \text { with } \bar{T}_{l}=0
\end{aligned}
$$




\subsubsection{Experiment}

\subsubsection{Subjects}

Six healthy subjects $(75.6 \pm 10.3 \mathrm{~kg}, 181 \pm 9 \mathrm{~cm})$ participated in this experiment. All subjects read and signed an 'informed consent', which explained the objectives and experimental protocol.

\subsubsection{Experimental setup}

Experiments were performed with an adjustable instrumented wheelchair (modified IBIS Comfort Wheelchair), which allows different body postures when adjusting the seat support and backrest (Fig. 2.3). The backrest contains two parts for independent support of the trunk at the lumbothoracic- and thoracic regions respectively. The seat and trunk supports are upholstered with $40 \mathrm{~mm}$ of industrial foam and adjustable relative to the global chair frame. The seat is divided into a front and back part for independent support of the thighs and pelvis respectively. An analogue balance was placed on the footrests to monitor the supported leg weight. Reflective markers were placed on the chair and selected anatomical landmarks. Two-dimensional chair configuration and body segment orientations were obtained using an infrared camera motion capturing system (VICON, Oxford, UK). Three markers were placed on the lower extremities including the Lateral Femoral Epicondylus (LFE), Anterior Superior Iliac Spine (ASIS) and Posterior Superior Iliac Spine (PSIS), one marker was placed within the lumbar 'joint' centre (LJC) and four markers were placed on the chair including the front seat (FS), back seat (BS), upper back support (UB) and lower back support (LB).
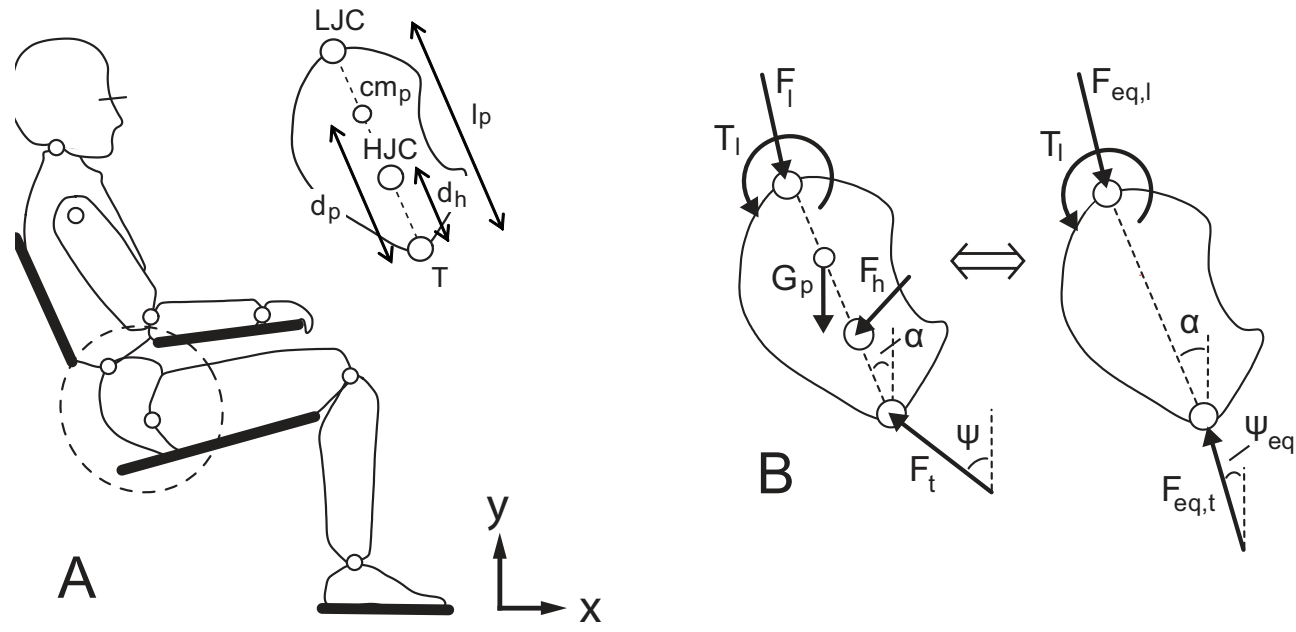

Figure 2.1: Equivalent 'two-force' member loading to estimate pelvis angle $(\alpha)$. A: Schematic representation of a seated person in the sagittal plane. Support of the trunk above the lumbosacral spine makes the pelvis function as the foundation for trunk support guiding the forces of the upper body to the seat. Hip joint centre (HJC) and the centre of pelvis mass $\left(\mathrm{cm}_{\mathrm{p}}\right)$ lie on the line connecting the lumbar joint centre (LJC) and tuberosities (T). B: The equivalent contact forces $\left(\mathrm{F}_{\text {eq, }}\right.$ and $\left.\mathrm{F}_{\text {eq, }}\right)$ substitute all forces $\left(\mathrm{F}_{\mathrm{l}}, \mathrm{F}_{\mathrm{h}}, \mathrm{F}_{\mathrm{t}}\right.$ and $\left.\mathrm{G}_{\mathrm{p}}\right)$ on the pelvis. The orientation of $\mathrm{F}_{\mathrm{eq}, \mathrm{t}}\left(\psi_{\mathrm{eq}}\right)$ equals $\alpha$ in the absence of lumbar torque $\left(\mathrm{T}_{1}\right)$. 

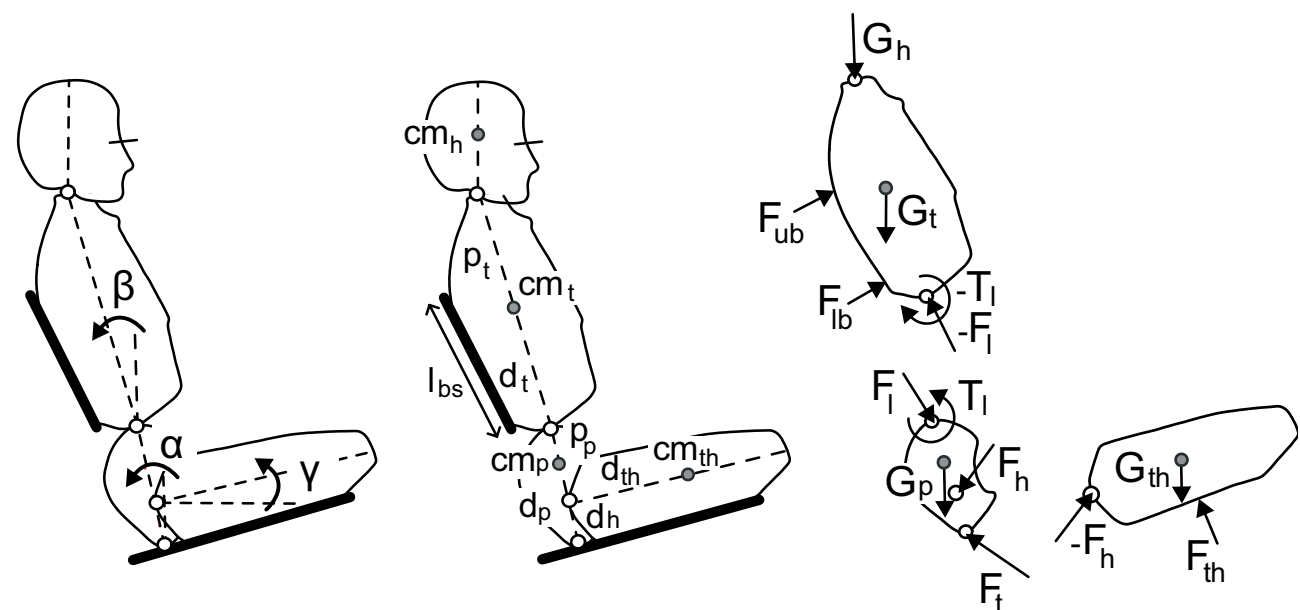

Figure 2.2: Rigid body model consisting of four body segments (head, trunk, pelvis and thighs) and two supporting areas (seat and back support). Positive body segmental angles ( $\alpha, \beta$, and $\gamma$ ), body dimensions and all body forces are shown.

Table 2.1: Body segment dimensions and masses of an average male subject.

\begin{tabular}{lll} 
segment & parameter & value \\
\hline \multirow{2}{*}{ head } & $\mathrm{m}_{\mathrm{h}}$ & $0.80 \mathrm{M}$ \\
& $\mathrm{p}_{\mathrm{t}}$ & $0.34 \mathrm{M}$ \\
& $\mathrm{l}_{\mathrm{bs}}$ & $0.13 \mathrm{~L}$ \\
& $\mathrm{~d}_{\mathrm{t}}$ & $0.18 \mathrm{~L}$ \\
\hline \multirow{2}{*}{ pelvis } & $\mathrm{m}_{\mathrm{p}}$ & $0.11 \mathrm{M}$ \\
& $\mathrm{p}_{\mathrm{p}}$ & $0.05 \mathrm{~L}$ \\
& $\mathrm{~d}_{\mathrm{p}}$ & $0.09 \mathrm{~L}$ \\
thighs & $\mathrm{p}_{\mathrm{h}}$ & $0.04 \mathrm{~L}$ \\
\hline & $\mathrm{m}_{\mathrm{th}}$ & $0.22 \mathrm{M}$ \\
& $\mathrm{d}_{\mathrm{th}}$ & $0.11 \mathrm{~L}$
\end{tabular}

body weight $(\mathrm{M})=80[\mathrm{~kg}]$, body length $(\mathrm{L})=1.8[\mathrm{~m}]$

For independent measurement of support forces under the pelvis and thighs, two multi-axis load cells (ATI mini 45, ATI Industrial Automation, NYC, USA) are mounted under each seat support. The location and orientation of the loads cells under the front and back seat support were identified by FS and BS respectively.

\subsubsection{Data analysis}

Sagittal two-dimensional data analysis was done using dedicated software implementation. Body segment orientation, chair configuration and seat support 

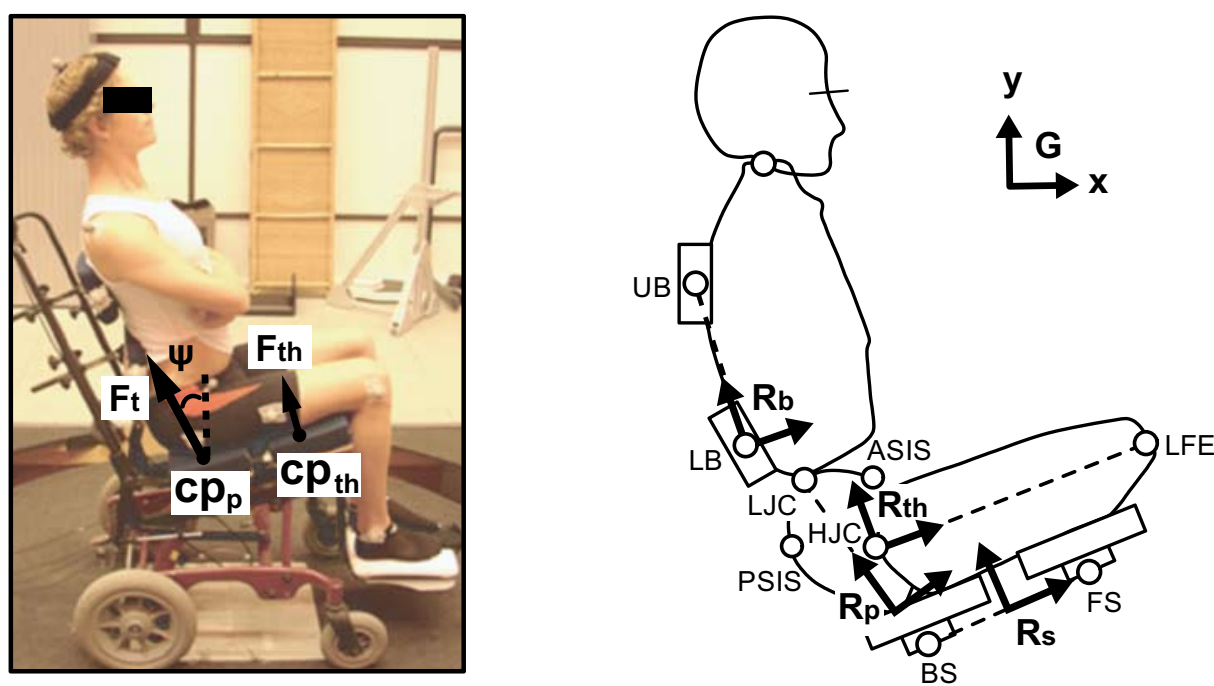

Figure 2.3: Experimental setup. Sagittal view of the instrumented wheelchair with adjustable seat and back supports. The seat is divided into a front and back part. Two multi-axis load cells are mounted under each part for measurement of the support forces and centre of pressure under the pelvis $\left(\mathrm{F}_{\mathrm{t}}\right.$ and $\left.\mathrm{cp}_{\mathrm{p}}\right)$ and thighs $\left(\mathrm{F}_{\mathrm{th}}\right.$ and $\left.\mathrm{cp}_{\mathrm{th}}\right)$. Reflective markers were placed on the chair (UB, LB, BS and FS) and selected anatomical landmarks (PSIS, ASIS and LFE). Chair configuration and body segment orientations were obtained using an infrared motion capturing analyses system. Local reference frames $\left(R_{s}, R_{p}, R_{b}\right.$ and $\left.R_{t h}\right)$ were constructed to compute chair configuration and body segment orientations.

forces were expressed relative to a global reference frame $(G)$ that aligned the anatomical sagittal plane of the subject. The support forces and centre of pressure under the pelvis $\left(\mathrm{F}_{\mathrm{t}}\right.$ and $\left.\mathrm{cp}_{\mathrm{p}}\right)$ and thighs $\left(\mathrm{F}_{\mathrm{th}}\right.$ and $\left.\mathrm{cp}_{\mathrm{th}}\right)$ were derived from the load cells that are mounted under the back and the front part of the seat respectively. The local backrest frame $\left(R_{b}\right)$ was constructed from LB and UB, the local seat frame $\left(R_{s}\right)$ from $\mathrm{BS}$ and FS, and the local pelvis frame $\left(\mathrm{R}_{\mathrm{p}}\right)$ from $\mathrm{cp}_{\mathrm{p}}$ and LJC. The location of the hip joint centre (HJC) was estimated from pelvic width (distance between the left and right ASIS) according to Bell et al. (Bell et al., 1990). The local thigh frame $\left(\mathrm{R}_{\mathrm{th}}\right)$ was constructed from HJC and LFE. Pelvis angle $(\alpha)$, backrest angle $(\beta)$, thigh angle $(\gamma)$ and seat angle $(\phi)$ defined orientations of $R_{p}, R_{b}, R_{t h}$ and $R_{s}$ relative to $G$ respectively (Fig. 2.3). Nett lumbar joint forces $\left(\mathrm{F}_{1}\right)$ and torques $\left(\mathrm{T}_{1}\right)$ where computed relative to LJC using static equations of equilibrium for the pelvis and thighs. Orientations of $F_{t}(\psi)$ and $\mathrm{F}_{\mathrm{eq}, \mathrm{t}}\left(\psi_{\mathrm{eq}}\right)$ were computed and related to $\alpha$.

\subsubsection{Experimental protocol}

The experiment contained five trails, each evaluating body posture for different tilt-inspace chair angles. As shown in figure 2.4, trial 1-5 involved stepwise lumbothoracic trunk support adjustments for different tilt-in-space chair angles (resp. $0^{\circ}, 5^{\circ}, 10^{\circ}$, $15^{\circ}$ and $20^{\circ}$ ). Before every trial, an empty seated force calibration zero-measurement was performed. Subjects were seated with their tuberosities on the back seat support. The footrests were set perpendicular to the ground so that the analogue balance monitored the estimated weight of the lower legs and no joint forces acted in the knees. Each trial started with an initial body posture of maximal pelvis anterior tilt and 


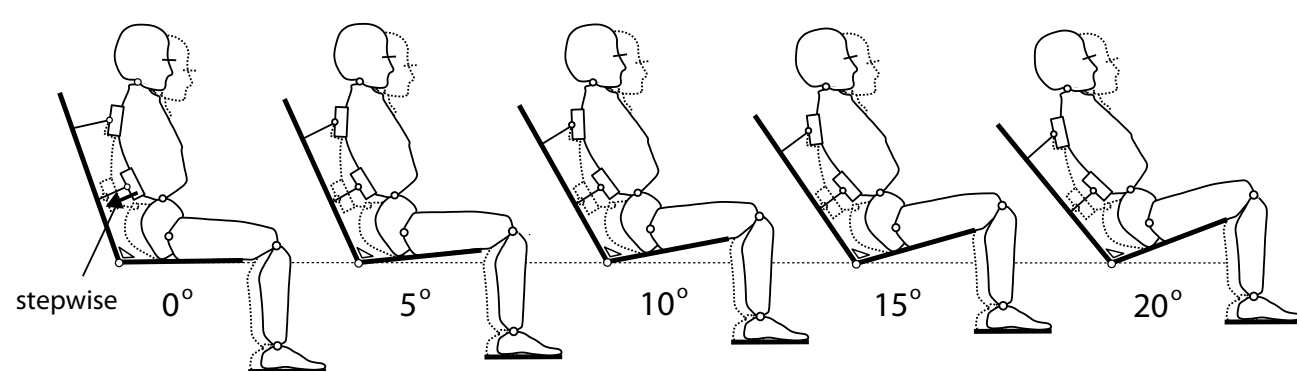

Figure 2.4: The experimental protocol involved stepwise lumbothoracic trunk support adjustments for different tilt-in-space chair angles (resp. $0^{\circ}, 5^{\circ}, 10^{\circ}, 15^{\circ}$ and $20^{\circ}$ ).

maximal lumbar lordosis, which was imposed by adjustment of the back supports. Subjects were asked to keep their arms folded, maintain a vertical head orientation and to adopt body posture is a passive way. This latter meant that they were asked to minimize any muscle function that could influence body posture as imposed by the configuration of the chair. To adjust body posture in each trail, the lower back support was translated backward stepwise until a final posture was adopted with maximal pelvis posterior tilt. Each trial took approximately 15 minutes. In-between adjustments (stepwise) of the lumbar support, static body postures were adopted for two minutes. Data collection only occurred during the last 60 seconds. Chair configuration, body segment orientation and seat support forces were captured and stored for offline analysis.

\subsection{Results}

\subsubsection{Model simulations}

Pelvis angle estimation ( $\hat{\alpha})$ from orientations of $F_{t}(\psi), F_{1}\left(\psi_{1}\right), F_{2}\left(\psi_{2}\right)$ and $F_{\text {eq, }}\left(\psi_{\text {eq }}\right)$ are shown in figure 2.5A-D respectively. The identity lines (oblique dashed lines) refer to situations in which $\alpha$ equals $\hat{\alpha}$. Figure 2.5A shows the relation between $\alpha$ and $\hat{\alpha}$ derived from the orientation of $F_{t}(\psi)$. Thigh $(\gamma)$ and trunk $(\beta)$ angles were set to $12^{\circ}\left(\gamma_{2}\right)$ and $24^{\circ}$ respectively. Great difference between $\psi$ and the identity line indicates that $\alpha$ could not directly be estimated from $\mathrm{F}_{\mathrm{t}}$. Figure $2.5 \mathrm{~B}$ shows the individual influence of $F_{h}$ on $\hat{\alpha}$. Thigh angle $\left(\gamma_{1-4}\right)$ affected $F_{h}$ and influenced $\hat{\alpha}$ that we derived from the orientation of $F_{1}\left(\psi_{1}\right)$. Differences in offset are observed. The influence of pelvis mass $\left(G_{p}\right)$ is predicted in figure 2.5C. A small slope difference is shown between the orientation of $\mathrm{F}_{2}\left(\psi_{2}\right)$ and the identity line. Figure 2.5D reflects the influence of lumbar torque $\left(\mathrm{T}_{1}\right)$ on the relation between $\alpha$ and $\hat{\alpha}$, which was derived from the orientation of $\mathrm{F}_{\text {eq, }}\left(\psi_{\text {eq }}\right)$. The identity line coincides $\psi_{\text {eq }}$ when no lumbar torque is present $\left(\mathrm{T}_{1}=\right.$ $0 \mathrm{Nm})$. In this situation, $\alpha$ could directly be predicted from $\psi_{\text {eq. }}$.

\subsubsection{Experimental results}

Figure 2.6A and 2.6B show the relations (third order best-fit) between the actual pelvis angle $(\alpha)$ and the predicted $(\hat{\alpha})$ pelvis angle derived from the orientations of $F_{t}$ $(\psi)$ and $\mathrm{F}_{\text {eq, }}\left(\psi_{\text {eq }}\right)$ respectively. The identity lines reflect the situation when $\alpha$ equals $\hat{\alpha}$ 
. For both situations, no clear relation was found between $\alpha$ and $\hat{\alpha}$ which indicates that pelvis angle could not directly be estimate from $\mathrm{F}_{\mathrm{t}}$ and $\mathrm{F}_{\mathrm{eq}, \mathrm{t}^{*}}$

\subsection{Discussion}

Pelvis alignment directly affects sitting posture and systems that control pelvis angle might be applicable to regulate body posture in long-term sitting. To use pelvis angle as feedback for proper posture control, interventions are needed that adjust and
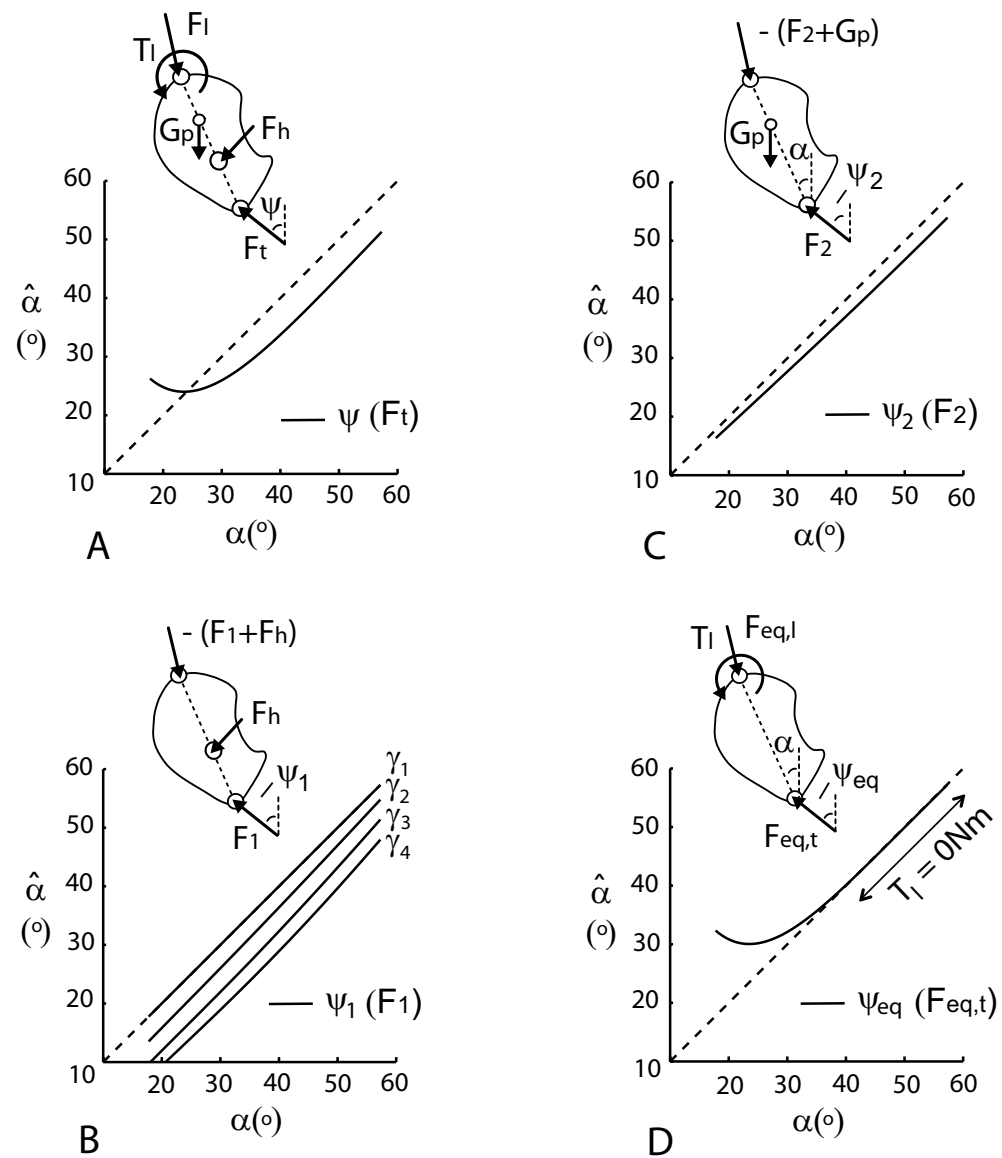

Figure 2.5: Pelvis angle estimation ( $\hat{\alpha})$ from orientations of $F_{t}(\psi), F_{1}\left(\psi_{1}\right), F_{2}\left(\psi_{2}\right)$ and $F_{\text {eq, }}\left(\psi_{\text {eq }}\right)$ respectively. The identity lines (oblique dashed lines) refer to situations in which $\alpha$ equals $\hat{\alpha}$. A: The relation between $\alpha$ and $\hat{\alpha}$ derived from the orientation of $F_{t}(\psi)$. B: influence of hip force (Fh) on $\hat{\alpha}$. Thigh angle $\left(\gamma_{1-4}\right)$ affected $F_{h}$ and influenced that was derived from the orientation of $F_{1}\left(\psi_{1}\right)$. C: influence of pelvis mass $\left(G_{p}\right)$ on derived from the orientation of $F_{2}\left(\psi_{2}\right)$. D: influence of lumbar torque $\left(\mathrm{T}_{1}\right)$ on derived from the orientation of $\mathrm{F}_{\text {eq, }}\left(\psi_{\mathrm{eq}}\right)$. The identity line coincides $\psi_{\text {eq }}$ when no lumbar torque is present $\left(\mathrm{T}_{1}=0 \mathrm{Nm}\right)$. 

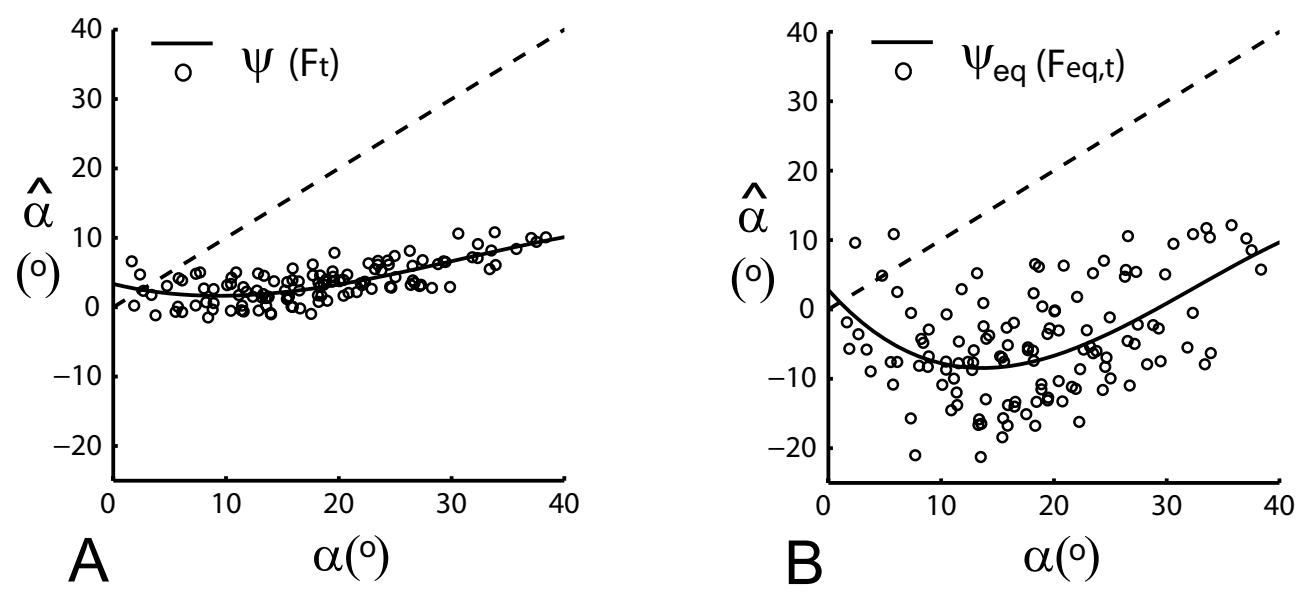

Figure 2.6: Relations (third order best-fit) between the predicted pelvis angle $(\hat{\alpha})$ and the actual pelvis angle $(\alpha)$ derived from orientations of $F_{t}(\psi)$ and Feq, $t\left(\psi_{\text {eq }}\right)$ respectively. For the identity lines (oblique dashed lines), $\alpha$ equals $\hat{\alpha}$.

monitor pelvis angle simultaneously. In a previous study (van Geffen et al., 2008), we described a seating system that effectively adjusts the pelvis independent from the trunk and seat support. To monitor pelvis alignment for proper pelvis control, the present study explored whether it is feasible to predict pelvis angle from seat support forces based on a theoretical analysis and measurements for experimental validation.

The estimation of pelvis angle was based on equivalent 'two-force member' loading in which pelvis angle equals the orientation of the equivalent contact force. When the backrest supports the trunk above the lumbosacral spine, forces of the upper body are guided through the pelvis to the seat. When no other forces exert on the pelvis, the orientation of the tuberal contact force equals pelvis orientation. However, pelvis mass, hip force and lumbar torque 'disturb' this condition and complicate the estimation of pelvis angle.

The theoretical analysis showed significant influence of pelvis mass, hip force and lumbar torque on the relation between the actual pelvis angle and the predicted pelvis angle. The tuberal contact force had to be compensated to derive the equivalent contact force from which pelvis angle could be estimated. The orientation of the equivalent contact force equals pelvis angle in the absence of lumbar torque. Therefore, it is only possible to derive pelvis angle when lumbar torque is minimal or can be prevented. The passive lumbar stiffness that was implemented during model simulation created zero lumbar torque in body postures with varying pelvis alignment (Fig. 2.5D). Because this suggested that pelvis angle might be predictable for a large range of body postures, validation experiments have been performed.

Experiments were done with an instrumented wheelchair that allows different body postures when adjusting the configuration of the seat and back support. Load cells are integrated in the seat for independent measurement of support forces under the pelvis and thighs. No body postures were found in which pelvis angle could directly be estimated from the orientation of the equivalent contact force. Significant lumbar torque was measured in all seating conditions which could indicate that healthy 
individuals continuously used their pelvis muscles even after they were instructed to minimize any muscle function that could influence body posture as imposed by the configuration of the chair. Because lumbar torque directly affects the forces at the lower back, healthy individuals might use their pelvis muscles to regulate the mechanical lumbar loading conditions in spinal postures that are associated with lumbar discomfort and pain (Hedman and Fernie, 1997, O'Sullivan et al., 2006). Besides active muscle control, the model to predict pelvis angle does also not include deformation of the buttock tissue and other pelvis structures. Although it is hard to predict what the effects would be during postural adjustments, a model that includes buttock tissue characteristics and pelvis muscle contraction might improve the prediction of pelvis angle.

We concluded that pelvis angle could not be estimated in healthy individuals under the described experimental seating conditions. Validation experiments on impaired individuals under other seating conditions and with less model simplifications might change this point of view and provide a better understanding whether the principle would be of interest for clinical application.

\subsection{Acknowledgements}

This study was partly funded by the Dutch Ministry of Economic Affairs, SenterNovem. The authors would like to thank Bert Faber (Welzorg Special Products, Oldenzaal, the Netherlands) for modification of the experimental chair and sharing his expertise in seating ergonomics. The authors also thank Mireille Florijn for her efforts during this study. 


\section{Chapter 3}

\section{Body Segments Decoupling to Control Sitting Posture}

Published as: P. van Geffen, B.I. Molier, J. Reenalda, P.H. Veltink and H.F.J.M. Koopman, 2008, Body segments decoupling in sitting: Control of body posture from automatic chair adjustments. Journal of Biomechanics, Volume 41, Issue 16, Pages 3419 - 3425. 


\begin{abstract}
Individuals who cannot functionally reposition themselves adopt a passive body posture and suffer from physical discomfort in long-term sitting. To regulate body load and to prevent sitting related mobility problems, proper postural control is important. The inability to reposition underlines the importance for seating interventions that control body posture from automatic chair adjustments. We developed an adjustable simulator chair that allows the alignment of the trunk, pelvis and thighs to be controlled independently. This study describes the system for decoupled body segments adjustment and develops a predictive model that computes angular chair configuration for desired body postures.

Eighteen healthy male subjects participated in this study. The experiment involved a protocol of five trials, each investigating the effect of individual chair segment angle adjustment on body segments rotation. Quasi-static chair adjustments were performed, in which angular chair configuration and body segments orientation were measured using an infrared motion capturing system and an inertial sensor attached on the pelvis. Linear best-fit equations together with the coefficients of determination were computed.

Significant relations have been found between angular chair configuration and body segments orientation leading to an algorithm that predicts chair configuration for desired body posture.

The predictive algorithm seems applicable to compute angular chair configuration for desired body posture when the initial body-chair configuration in known. For clinical application, future experiments must be performed on impaired individuals to validate the algorithm in terms of accuracy.
\end{abstract}




\subsection{Introduction}

Individuals who cannot functionally reposition themselves in long-term sitting adopt a passive body posture and often suffer from physical discomfort such as pressure ulcers (Collins, 1999), low back injury (Verver et al., 2003, Lengsfeld et al., 2000a, Lengsfeld et al., 2000b, Makhsous et al., 2003, van Deursen et al., 2000, Ferguson and Marras, 1997), respiratory dysfunction (Lin et al., 2006), lumbar immobility and joint stiffness (Beach et al., 2005). It has been accepted with some certainty that most physical problems are associated with sustained mechanical body loading and that dynamic seating interventions are needed to periodically adjust body posture associated with wheelchair discomfort (Crane et al., 2007).

Able-bodied individuals do not suffer mobility problems since they continuously shift body posture. In a recent study, Linder-Ganz et al. evaluated healthy sitting behaviour and reported significant movement of the upper extremities, trunk and pelvis when quantifying postural change in prolonged wheelchair sitting of healthy individuals (Linder-Ganz et al., 2007). Because body posture is mainly determined by the alignment of the trunk, pelvis and thighs, proper posture control involves the ability to move all three body segments independently. However, for individuals who cannot functionally reposition themselves, no applications are yet known that allows decoupled trunk, pelvis and thighs adjustments.

Classical wheelchairs with one pivot between the seat and backrest make it impossible to adjust the trunk, pelvis and thighs independently and introduce sliding when changing its configuration (Aissaoui et al., 2001). We therefore designed a chair that allows decoupled alignment of the trunk, pelvis and thighs by aligning the axes for angular chair adjustments with the axes for body segments rotation.

This study describes the system for decoupled body segments adjustment and develops a predictive model that computes angular chair configuration for desired body postures.

\subsection{Methods}

This study was approved by the local Committee for Medical Ethics of Rehabilitation Centre 't Roessingh (Enschede, the Netherlands).

\subsubsection{Subjects}

Eighteen healthy male subjects (age $22.6 \pm 2.4$ years, weight $74.9 \pm 8.0 \mathrm{~kg}$, length $1.84 \pm 0.05 \mathrm{~m}$, BMI $22.1 \pm 1.9 \mathrm{~kg} / \mathrm{m}^{2}$ ) were recruited for this study. All subjects read and signed an 'informed consent', which explained the objective and experimental protocol.

\subsubsection{Decoupled body segments adjustment}

A 'classical' chair with one pivot between the seat and backrest makes it impossible to adjust the trunk, pelvis and thighs independently and introduces sliding when changing its configuration (Fig 3.1A). A concept with the axes of rotation aligned within the lumbar region and under the ischial tuberosities allows independent body 
segments adjustment in the sagittal plane (Fig. 3.1B). Based on a parallelogram from which the backrest is actuated, a mechanical concept for decoupled body segments adjustment is shown in figure 3.1C. Actuating the configuration of the parallelogram rotates the pelvis in the sagittal plane. The whole mechanism rotates around a pivot under the tuberosities and is externally actuated. In the frontal plane, actuating the relative height between the left and right seat part rotates the pelvis sideways around an axis between the ischial tuberosities. For lateral trunk rotation, the backrest is actuated around a pivot within the lumbothoracic region.

\subsubsection{Experimental setup}

A fully adjustable computer-aided simulator chair was developed which contained the concept for decoupled body segments adjustment (Fig. 3.2). The backrest is mounted on a sledge to allow vertical trunk translations and to align the axes for body segments rotation exactly with the axes for chair adjustment. The weight of the backrest is counterbalanced.

The backrest, seat, parallelogram and footrests are adjustable to align body posture with chair configuration. The parallelogram height and backrest depth were set in a way that the axis for sagittal backrest adjustment would be around the middle of the lumbar spine. This was determined from pilot experiments on healthy male subject with posture characteristics similar to those recruited for the present study.

Reflective markers were placed on the chair and selected anatomical landmarks. Three-dimensional chair configuration and body segments orientation were obtained using an infrared camera motion capturing system (VICON ${ }^{\circledR}$, Oxford, UK). To prevent skin artifacts during pelvis movement, a pelvis mold (PM) was shaped around the left and right lateral iliac crest and clamped the Anterior Superior Iliac Spine and

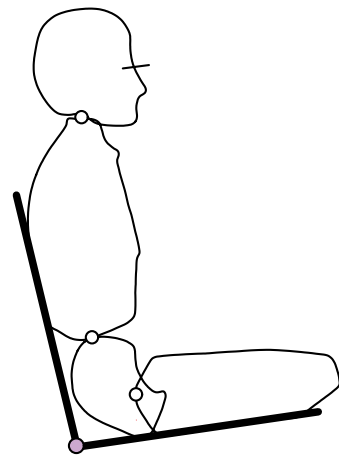

A

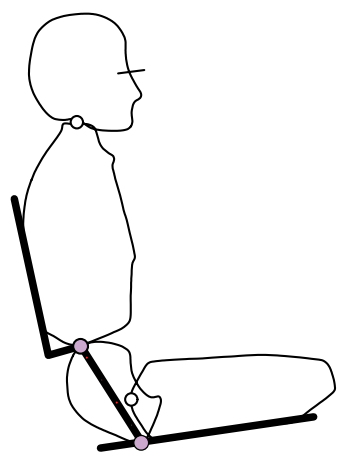

B

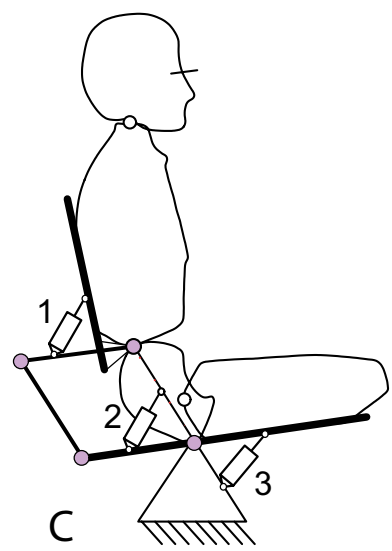

Figure 3.1: Principle for sagittal postural adjustments. A: Classical chairs with one pivot between the seat and backrest do not allow independent trunk, pelvis and thighs adjustments. B: Concept with two axes of rotation aligned within the lumbar region and under the ischial tuberosities enables independent body segments control in the sagittal plane. C: Based on a parallelogram, a mechanical concept for decoupled body segments adjustment is shown. The backrest is adjusted from the parallelogram (1), the parallelogram from the back seat (2) and the seat from the under frame (3). 
Posterior Superior Iliac Spines for optimal fixation (Fig. 3.3). To prevent problems with pelvis marker visibility, an inertial sensor (MT ${ }^{\circledR}$, Xsens, Enschede, the Netherlands) was attached on the PM as an alternative to estimate pelvis orientation. The line of gravity was used to derive sensor inclination that we related to the orientation of the $\mathrm{PM}$ as shown in figure 3.3.

A pressure mapping device (Tekscan ${ }^{\circledR}$, Boston, USA) was placed over the seat to locate the position of the tuberosities.

\section{sagittal}

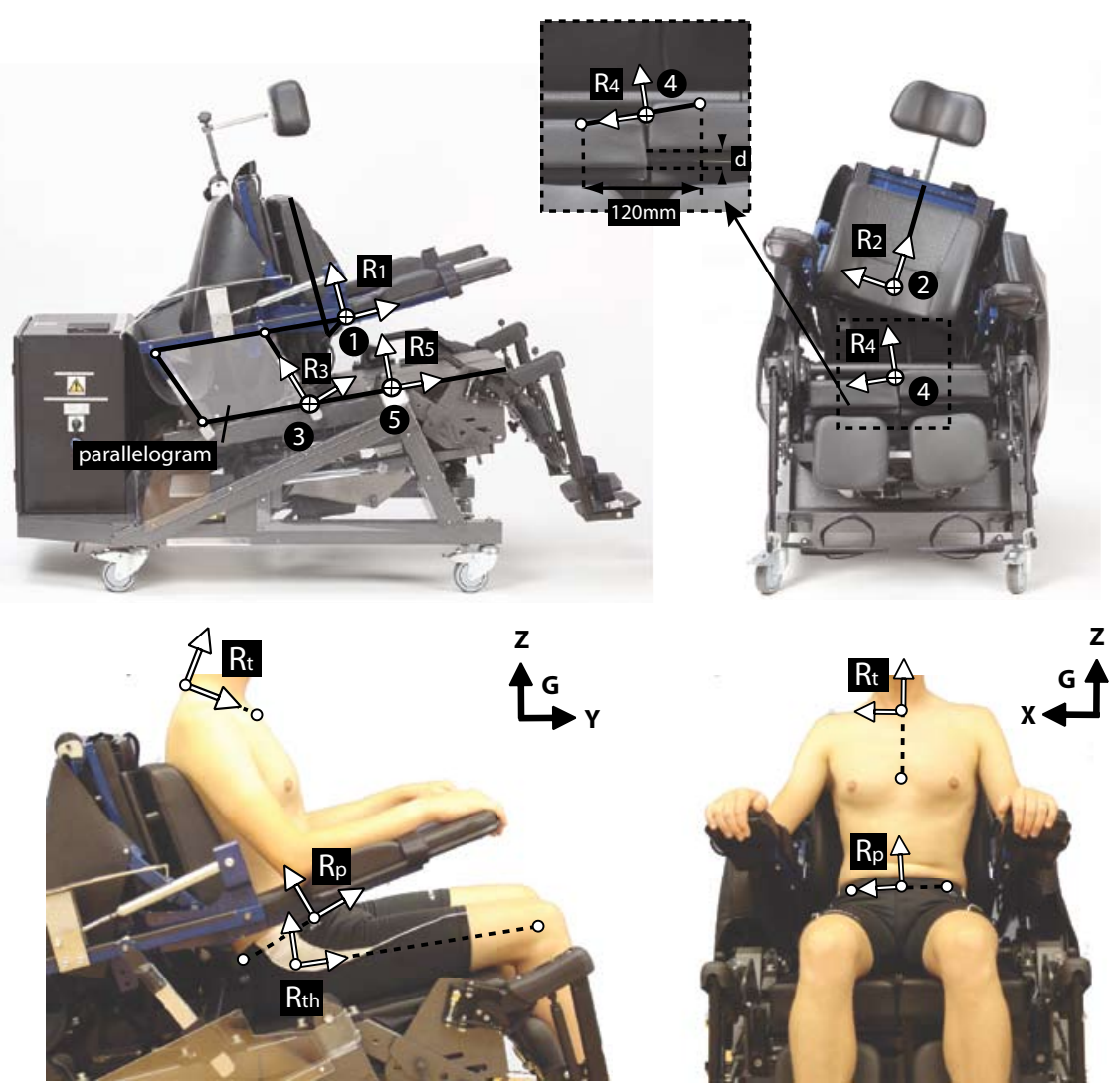

Figure 3.2: Sagittal and frontal view of the computer-aided adjustable simulator chair instrumented with the concepts for decoupled body segments adjustment. The axes of rotation (1-5) together with the local reference frames $\left(\mathrm{R}_{1}-\mathrm{R}_{5}\right)$ for all chair segments adjustments are shown. $R_{1}, R_{3}$ and $R_{5}$ define the frames for the backrest, parallelogram and seat in the sagittal plane. $R_{2}$ and $R_{4}$ define the frames for the backrest and seat in the frontal plane. $R_{4}$ is constructed from the height $\mathrm{d}[\mathrm{mm}]$ between the left and right seat part assuming an average ischial tuberal width of $120 \mathrm{~mm}$. G defines the global reference frame. $R_{t}, R_{p}$ and $R_{t h}$ reflect the local frames for the trunk, pelvis and thighs. 


\subsubsection{Reference coordinate frames and joint centre locations}

The $x-z$ and $y-z$ planes of the global reference frame $(G)$ aligned respectively the frontal and sagittal planes of the simulator chair.

The local trunk frame $\left(\mathrm{R}_{\mathrm{t}}\right)$ was constructed from the $7^{\text {th }}$ Cervical $(\mathrm{C} 7)$, Upper Sternum (US) and Processus Xypoid (PX). The y-axis of $\mathrm{R}_{\mathrm{t}}$ runs from $\mathrm{C} 7$ to US. The $\mathrm{x}$-axis points right and is normal to the plane containing C7, US and PX. The z-axis is the cross product of the $x$ - and $y$-axis. The local pelvis frame $\left(R_{p}\right)$ was constructed from four markers placed on the PM. Two markers were placed on the left and right Anterior Superior Iliac Spines (LASIS and RASIS) and two markers (LSIS and RSIS) on the line between the Anterior- and Posterior Superior Iliac Spines. The $\mathrm{x}$-axis of $\mathrm{R}_{\mathrm{p}}$ runs from the LASIS to the RASIS. The z-axis points cranially and is normal to the plane containing the LASIS, RASIS and the midpoint between LSIS and RSIS. The y-axis is the cross product of the $\mathrm{z}$ - and $\mathrm{x}$-axis. The midpoint of RASIS and LASIS defined the origin of $\mathrm{R}_{\mathrm{p}}$. Local coordinates for the left and right hip joint centers (HJCs) were estimated from pelvic width (distance between RASIS and LASIS) and expressed in $\mathrm{R}_{\mathrm{p}}$ according to the regression equations as reported by Bell et al. (Bell et al., 1990) In case of problems with pelvis marker visibility, information from the inertial sensor was used to construct a local sensor frame $\left(\mathrm{R}_{\mathrm{i}}\right)$ that we related to $\mathrm{R}_{\mathrm{p}}$ (Fig. 3.3). The local coordinate frames for the thighs $\left(\mathrm{R}_{\mathrm{th}}\right)$ are constructed from the HJCs, Lateral Femoral Epicondyles (LFEs) and Medial Femoral Epicondyles (MFEs). The knee joint centers (KJCs) are estimated at the midpoint between the LFEs and MFEs. The y-axis of $\mathrm{R}_{\mathrm{th}}$ runs from the HJCs to the KJCs. The z-axis points cranially and is normal to the plane containing the HJCs, LFEs and MFEs. The $\mathrm{x}$-axis is the cross product of the $\mathrm{y}$ - and z-axes.

In the sagittal plane, the local coordinate frames for the backrest $\left(\mathrm{R}_{1}\right)$, parallelogram
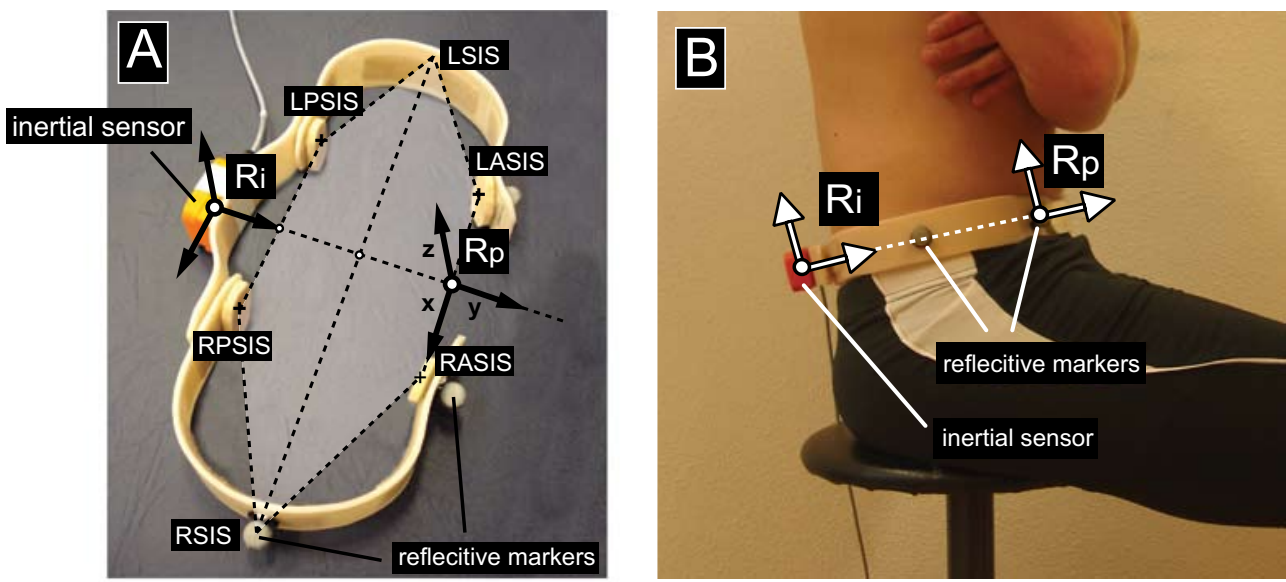

Figure 3.3: A: Pelvis mold with reflective markers and inertial sensor. The local pelvis frame $\left(\mathrm{R}_{\mathrm{p}}\right)$ is constructed from the reflective markers that were placed on the RASIS, LASIS, RSIS and LSIS. The RPSIS and LPSIS lie in the $x-y$ plane. The inertial sensor is attached in a way that the local sensor frame $\left(\mathrm{R}_{\mathrm{i}}\right)$ aligns $\mathrm{R}_{\mathrm{p}}$. B: The pelvis mold clamped the RASIS, LASIS, RPSIS and LPSIS and was shaped around the left and right lateral iliac crest. $\mathrm{R}_{\mathrm{i}}$ was used as an alternative measure for $R_{p}$ when the reflective pelvis markers were not visible. 
$\left(\mathrm{R}_{3}\right)$ and seat support $\left(\mathrm{R}_{5}\right)$ were constructed from angular chair configuration as shown in figure 3.2. In the frontal plane, the local backrest frame $\left(\mathrm{R}_{2}\right)$ was constructed from angular backrest adjustment. Figure 3.2 also shows how we constructed a local coordinate frame $\left(\mathrm{R}_{4}\right)$ from the contact areas under the ischial tuberosities. This was done from the angle $(\phi)$ that we derived from the height difference $\mathrm{d}[\mathrm{mm}]$ between the left and right seat part assuming an average ischial tuberal width of $120 \mathrm{~mm}$ (Linder-Ganz et al., 2008): $\tan (\phi)=\mathrm{d} / 120$.

\subsubsection{Body- and chair segments orientation}

Helical angles were computed for all body- and chair segments. In the sagittal plane, the orientations of $R_{t}, R_{p}$ and $R_{t h}$ relative to $G$ defined the sagittal body segments angles for the trunk $\left(\alpha_{x}\right)$, pelvis $\left(\beta_{x}\right)$ and thighs $\left(\gamma_{x}\right)$ respectively. The orientations of $R_{1}, R_{3}$ and $R_{5}$ relative to $G$ defined the sagittal chair segments angles for the backrest $\left(\mathrm{A}_{\mathrm{x}}\right)$, parallelogram $\left(\mathrm{B}_{\mathrm{x}}\right)$ and seat support $\left(\mathrm{H}_{\mathrm{x}}\right)$ respectively. In the frontal plane, the orientations of $R_{t}$ and $R_{p}$ relative to $G$ defined the frontal body segments angles for the trunk $\left(\alpha_{\mathrm{y}}\right)$ and the pelvis $\left(\beta_{\mathrm{y}}\right)$ respectively. The orientations of $R_{2}$ and $R_{4}$ relative to $G$ defined the frontal chair segments angles for the backrest $\left(\mathrm{A}_{\mathrm{y}}\right)$ and seat support $\left(\mathrm{H}_{\mathrm{y}}\right)$ respectively.

\subsubsection{Angular chair configuration vs. body segments orientation}

The experiment involved five trials (Fig. 3.4), each investigating the effect of one specific chair segment angle adjustment on the alignment of all body segments. Trial 1 - 5 concerned sagittal backrest angle adjustment (trial 1), frontal backrest angle adjustment (trial 2), sagittal parallelogram angle adjustment (trial 3), frontal seat part adjustment (trial 4) and sagittal seat angle adjustment (trial 5). Linear relations between angular chair configuration and body segments orientation were assumed. For every trial, we related angular chair adjustment to the alignment of all body segments and computed the linear best-fit equations together with the coefficients of correlation ( $r$ ) and accompanying significance (p). We then calculated the coefficients of determination $\left(\mathrm{r}^{2}\right)$ which gives us an indication about the strength of the relation. High coefficients of determination $\left(r^{2}>0.8\right)$ indicate strong relations. The computed linear equations were used to formulate the matrix equation (Eq. 1) in which each column of $\mathrm{C}$ was filled with the slope coefficients from the results of corresponding trial number.

$$
q=C \cdot u
$$

In which

$$
q=\left[\begin{array}{c}
\Delta \alpha_{x} \\
\Delta \alpha_{y} \\
\Delta \beta_{x} \\
\Delta \beta_{y} \\
\Delta \gamma_{x}
\end{array}\right]=\left[\begin{array}{c}
\alpha_{x}-\alpha_{x 0} \\
\alpha_{y}-\alpha_{y 0} \\
\beta_{x}-\beta_{x 0} \\
\beta_{y}-\beta_{y 0} \\
\gamma_{x}-\gamma_{x 0}
\end{array}\right] \quad u=\left[\begin{array}{c}
\Delta A_{x} \\
\Delta A_{y} \\
\Delta B_{x} \\
\Delta B_{y} \\
\Delta H_{x}
\end{array}\right]=\left[\begin{array}{c}
A_{x}-A_{x 0} \\
A_{y}-A_{y 0} \\
B_{x}-B_{x 0} \\
B_{y}-B_{y 0} \\
H_{x}-H_{x 0}
\end{array}\right] \quad \begin{aligned}
& C=\left[C_{i j}\right] \\
& i=j=1,5
\end{aligned}
$$


$\mathrm{A}_{\mathrm{x} 0} \ldots \mathrm{H}_{\mathrm{x} 0}$ and $\alpha_{\mathrm{x} 0} \ldots \gamma_{\mathrm{x} 0}$ define the initial segment angles for angular chair configuration and body posture respectively. Inverting $\mathrm{C}$ gives the equation that predicts angular chair configuration for desired body segment alignment.

\subsubsection{Experimental protocol}

Subjects were seated with their ischial tuberosities aligned with the pivots for sagittal seat inclination and pelvis rotation. Ischial peak pressure mapping (Tekscan ${ }^{\circledR}$ Comformat, Boston, USA) was used to monitor the ischial tuberosities when positioning the subject in the middle of the seat and aligning the anatomical sagittal plane with the global $y-z$ plane. The footrests were set perpendicular to the seat in a way that the thighs were supported by the seat except for the popliteal space. Reflective markers were placed and the initial angular chair configuration was set $\left(\mathrm{A}_{\mathrm{x} 0}=27.5^{\circ}, \mathrm{A}_{\mathrm{y} 0}=0^{\circ}, \mathrm{B}_{\mathrm{x} 0}=37.5^{\circ}, \mathrm{B}_{\mathrm{y} 0}=0^{\circ}\right.$ and $\left.\mathrm{H}_{\mathrm{x} 0}=12.5^{\circ}\right)$. Each trial started with a static reference measurement to relate the inertial sensor frame $\left(\mathrm{R}_{\mathrm{i}}\right)$ to the local pelvis frame $\left(R_{p}\right)$, and to define the initial body segments orientations $\left(\alpha_{x 0}, \alpha_{y 0}, \beta_{x 0}, \beta_{y 0}\right.$ and $\left.\gamma_{\mathrm{x} 0}\right)$. In the sagittal plane, the backrest angle was adjusted over a range of $30^{\circ}\left(\mathrm{A}_{\mathrm{x}}\right.$ $\left.=27.5^{\circ} \pm 15^{\circ}\right)$, the parallelogram angle over a range of $35^{\circ}\left(\mathrm{B}_{\mathrm{x}}=37.5 \pm 17.5^{\circ}\right)$ and the seat support angle over a range of $25^{\circ}\left(\mathrm{H}_{\mathrm{x}}=12.5 \pm 12.5^{\circ}\right)$. In the frontal plane, the backrest angle was adjusted over a range of $30^{\circ}\left(\mathrm{A}_{\mathrm{y}}=0 \pm 15^{\circ}\right)$ and the seat parts over a range of $60 \mathrm{~mm}(\mathrm{~d}=0 \pm 30 \mathrm{~mm})$.

Subjects were asked to undergo chair movements in a passive manner. This meant that they were not allowed to perform any activity that could influence body movement as induced by the chair. Each trial involved two minutes of quasi-static $(1 / 30 \mathrm{~Hz})$ chair adjustments. Data was only collected during the last 60 seconds.

\subsection{Results}

For every trial of individual angular chair segment adjustment $\left(\mathrm{A}_{\mathrm{x}}, \mathrm{A}_{\mathrm{y}}, \mathrm{B}_{\mathrm{x}}, \mathrm{B}_{\mathrm{y}}, \mathrm{H}_{\mathrm{x}}\right)$, the initial values and ranges for all body segment angles $\left(\alpha_{x}, \alpha_{y}, \beta_{x}, \beta_{y}, \gamma_{x}\right)$ are averaged over all subjects and shown in table 3.1.

As a selection of the results, figure 3.5 shows the results of the first trial which investigates the linear relations between sagittal backrest angle adjustment $\left(\Delta \mathrm{A}_{\mathrm{x}}\right)$ and all body segments rotations $\left(\Delta \alpha_{x}, \Delta \alpha_{y}, \Delta \beta_{x}, \Delta \beta_{y}, \Delta \gamma_{x}\right)$. Graphs show how the slope coefficients (c) and $\mathrm{r}^{2}$ are derived from the linear relations and give a good indication about the variability between subjects. This was done for all individual chair segment adjustments $\left(\Delta \mathrm{A}_{\mathrm{x}}, \Delta \mathrm{A}_{\mathrm{y}}, \Delta \mathrm{B}_{\mathrm{x}}, \Delta \mathrm{B}_{\mathrm{y}}\right.$ and $\left.\Delta \mathrm{H}_{\mathrm{x}}\right)$. Results for $\mathrm{c}$ and $\mathrm{r}^{2}$ are presented in table 3.2. The C-matrix that holds the linear effects of angular chair configuration $(\mathrm{u})$ on body segments orientation (q) was constructed from the mean slope coefficients (c), filling each column with the results from corresponding trial number (Fig. 3.6). The bold values in $C$ are accompanied with high coefficient of determination $\left(\mathrm{r}^{2}>0.8\right)$. It is shown that the slope coefficients of any significance (c $>0.1)$ all involve high values for $\mathrm{r}^{2}$. Inverting $\mathrm{C}$ gives the matrix equation that predicts angular chair configuration for desired posture alignment (Fig. 3.7). 

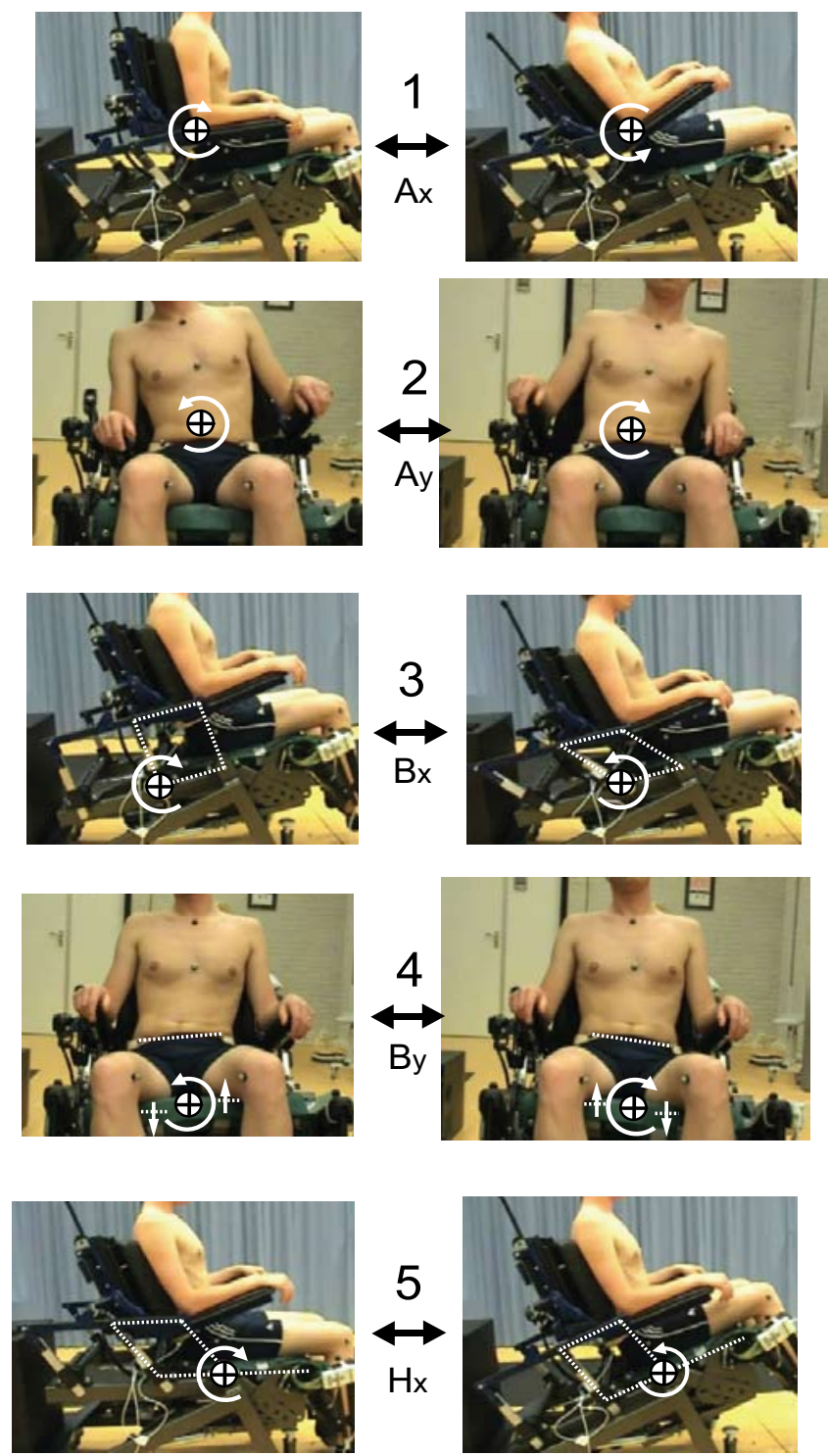

Figure 3.4: The experiment involved five trials (1-5), each investigating the effect of one specific chair adjustment on the alignment of all body segments. Trial 1: sagittal backrest angle adjustment $\left(\mathrm{A}_{\mathrm{x}}\right)$ around a pivot in the lumbar region. Trial 2: frontal backrest angle adjustment $\left(\mathrm{A}_{\mathrm{v}}\right)$ around a pivot in the lumbothoracic region. Trial 3: sagittal parallelogram angle adjustment $\left(B_{x}\right)$ around a pivot under the ischial tuberosities. Trial 4: frontal seat part adjustments $\left(B_{y}\right)$ which rotates the pelvis around a pivot between the ischial tuberosities. Trial 5: sagittal seat angle adjustment $\left(\mathrm{H}_{\mathrm{x}}\right)$ around a pivot under the ischial tuberosities. 


\subsection{Discussion}

To adjust body posture in the sagittal pane, the concept for body segments decoupling was based on a parallelogram that places the axis for trunk rotation within the lumbar region and for pelvis and thighs rotation under the ischial tuberosities. In the frontal plane, the pelvis rotates around a pivot between the ischial tuberosities from adjusting the height of the left and right seat parts. Lateral trunk rotation was

Table 3.1: Initial values and ranges for all body segment angles $\left(\alpha_{x}, \alpha_{y}, \beta_{x}, \beta_{y}, \gamma_{x}\right)$ in every trial of angular chair segment adjustment $\left(\mathrm{A}_{\mathrm{x}}, \mathrm{A}_{\mathrm{y}}, \mathrm{B}_{\mathrm{x}}, \mathrm{B}_{\mathrm{y}}, \mathrm{H}_{\mathrm{x}}\right)$.

\begin{tabular}{|c|c|c|c|c|c|c|}
\hline & & $A_{x}$ & $\mathrm{~A}_{\mathrm{y}}$ & $\mathrm{B}_{\mathrm{x}}$ & $\mathrm{B}_{\mathrm{y}}$ & $\mathrm{H}_{\mathrm{x}}$ \\
\hline \multirow{2}{*}{$\alpha_{x}$} & $\alpha_{\mathrm{x} 0}$ & $-22.7 \pm 6.0^{\circ}$ & $-23.5 \pm 5.0^{\circ}$ & $-21.4 \pm 5.9^{\circ}$ & $-23.5 \pm 5.7^{\circ}$ & $-23.2 \pm 5.7^{\circ}$ \\
\hline & range & $30.1 \pm 4.3^{\circ}$ & $0.4 \pm 2.3^{\circ}$ & $5.5 \pm 3.3^{\circ}$ & $0.4 \pm 0.7^{\circ}$ & $2.0 \pm 1.2^{\circ}$ \\
\hline \multirow{2}{*}{$\alpha_{y}$} & $\alpha_{\mathrm{y} 0}$ & $0.0 \pm 4.3^{\circ}$ & $-0.4 \pm 4.8^{\circ}$ & $-0.4 \pm 4.6^{\circ}$ & $-0.97 \pm 2.4^{\circ}$ & $-0.5 \pm 3.9^{\circ}$ \\
\hline & range & $0.6 \pm 1.6^{\circ}$ & $32.1 \pm 3.6^{\circ}$ & $0.4 \pm 0.8^{\circ}$ & $3.4 \pm 1.3^{\circ}$ & $0.2 \pm 0.6^{\circ}$ \\
\hline \multirow{2}{*}{$\beta_{\mathrm{x}}$} & $\beta_{\mathrm{x} 0}$ & $34.1 \pm 5.0^{\circ}$ & $32.9 \pm 5.2^{\circ}$ & $33.4 \pm 4.9^{\circ}$ & $35.5 \pm 5.1^{\circ}$ & $33.3 \pm 4.8^{\circ}$ \\
\hline & range & $6.0 \pm 1.7^{\circ}$ & $1.3 \pm 1.7^{\circ}$ & $19.7 \pm 2.4^{\circ}$ & $0.4 \pm 1.4^{\circ}$ & $4.6 \pm 2.3^{\circ}$ \\
\hline \multirow{2}{*}{$\beta_{\mathrm{y}}$} & $\beta_{\mathrm{y} 0}$ & $-1.8 \pm 2.0^{\circ}$ & $-1.6 \pm 1.9^{\circ}$ & $-1.7 \pm 2.1^{\circ}$ & $-1.0 \pm 2.5^{\circ}$ & $-1.3 \pm 2.3^{\circ}$ \\
\hline & range & $0.1 \pm 0.5^{\circ}$ & $7.2 \pm 3.3^{\circ}$ & $0.3 \pm 1.1^{\circ}$ & $9.8 \pm 1.2^{\circ}$ & $0.3 \pm 0.9^{\circ}$ \\
\hline \multirow[t]{2}{*}{$\gamma_{x}$} & $\gamma_{\mathrm{x} 0}$ & $2.1 \pm 2.9^{\circ}$ & $1.9 \pm 3.1^{\circ}$ & $2.5 \pm 3.6^{\circ}$ & $2.6 \pm 3.4^{\circ}$ & $2.5 \pm 3.0^{\circ}$ \\
\hline & range & $0.1 \pm 0.3^{\circ}$ & $1.2 \pm 0.6^{\circ}$ & $1.4 \pm 0.7^{\circ}$ & $1.1 \pm 0.4^{\circ}$ & $24.2 \pm 0.6^{\circ}$ \\
\hline
\end{tabular}

Table 3.2: Slope coefficients (c) and coefficients of determination $\left(\mathrm{r}^{2}\right)$ from the linear relations between angular chair segment adjustment $\left(\Delta \mathrm{A}_{\mathrm{x}}, \Delta \mathrm{A}_{\mathrm{y}}, \Delta \mathrm{B}_{\mathrm{x}}, \Delta \mathrm{B}_{\mathrm{y}}, \Delta \mathrm{H}_{\mathrm{x}}\right)$ and body segment rotation $\left(\Delta \alpha_{x}, \Delta \alpha_{y}, \Delta \beta_{x}, \Delta \beta_{y}, \Delta \gamma_{x}\right)$.

\begin{tabular}{lllllll} 
& & $\Delta \mathrm{A}_{\mathrm{x}}$ & $\Delta \mathrm{A}_{\mathrm{y}}$ & $\Delta \mathrm{B}_{\mathrm{x}}$ & $\Delta \mathrm{B}_{\mathrm{y}}$ & $\Delta \mathrm{H}_{\mathrm{x}}$ \\
\hline \multirow{2}{*}{$\Delta \alpha_{\mathrm{x}}$} & $\mathrm{c}$ & $1.00 \pm 0.14$ & $0.01 \pm 0.08$ & $0.15 \pm 0.09$ & $-0.02 \pm 0.02$ & $-0.08 \pm 0.05$ \\
& $\mathrm{r}^{2}$ & $0.99 \pm 0.01^{* * *}$ & $0.32 \pm 0.31^{* *}$ & $0.87 \pm 0.16^{* * *}$ & $0.32 \pm 0.21$ & $0.62 \pm 0.32^{* * *}$ \\
$\Delta \alpha_{\mathrm{y}}$ & с & $-0.02 \pm 0.05$ & $1.07 \pm 0.12$ & $-0.01 \pm 0.02$ & $-0.12 \pm 0.05$ & $0.01 \pm 0.02$ \\
& $\mathrm{r}^{2}$ & $0.70 \pm 0.31^{* * *}$ & $0.99 \pm 0.00^{* * *}$ & $0.40 \pm 0.36^{*}$ & $0.94 \pm 0.05^{* * *}$ & $0.31 \pm 0.25^{*}$ \\
$\Delta \beta_{\mathrm{x}}$ & $\mathrm{c}$ & $0.20 \pm 0.06$ & $-0.05 \pm 0.06$ & $0.52 \pm 0.06$ & $0.01 \pm 0.05$ & $0.18 \pm 0.09$ \\
& $\mathrm{r}^{2}$ & $0.97 \pm 0.01^{* * *}$ & $0.53 \pm 0.29^{* * *}$ & $0.99 \pm 0.00^{* * *}$ & $0.48 \pm 0.33^{* * *}$ & $0.87 \pm 0.16^{* * *}$ \\
$\Delta \beta_{\mathrm{y}}$ & с & $0.00 \pm 0.02$ & $0.24 \pm 0.11$ & $0.01 \pm 0.03$ & $0.35 \pm 0.04$ & $-0.01 \pm 0.04$ \\
& $\mathrm{r}^{2}$ & $0.58 \pm 0.36^{* * *}$ & $0.95 \pm 0.03^{* * *}$ & $0.85 \pm 0.19^{* * *}$ & $0.99 \pm 0.01^{* * *}$ & $0.64 \pm 0.31^{* * *}$ \\
$\Delta \gamma_{\mathrm{x}}$ & с & $0.00 \pm 0.01$ & $0.04 \pm 0.02$ & $0.04 \pm 0.02$ & $0.04 \pm 0.02$ & $0.97 \pm 0.02$
\end{tabular}

Significant correlations: * $\mathrm{p}<0.05,{ }^{* *} \mathrm{p}<0.01$ 

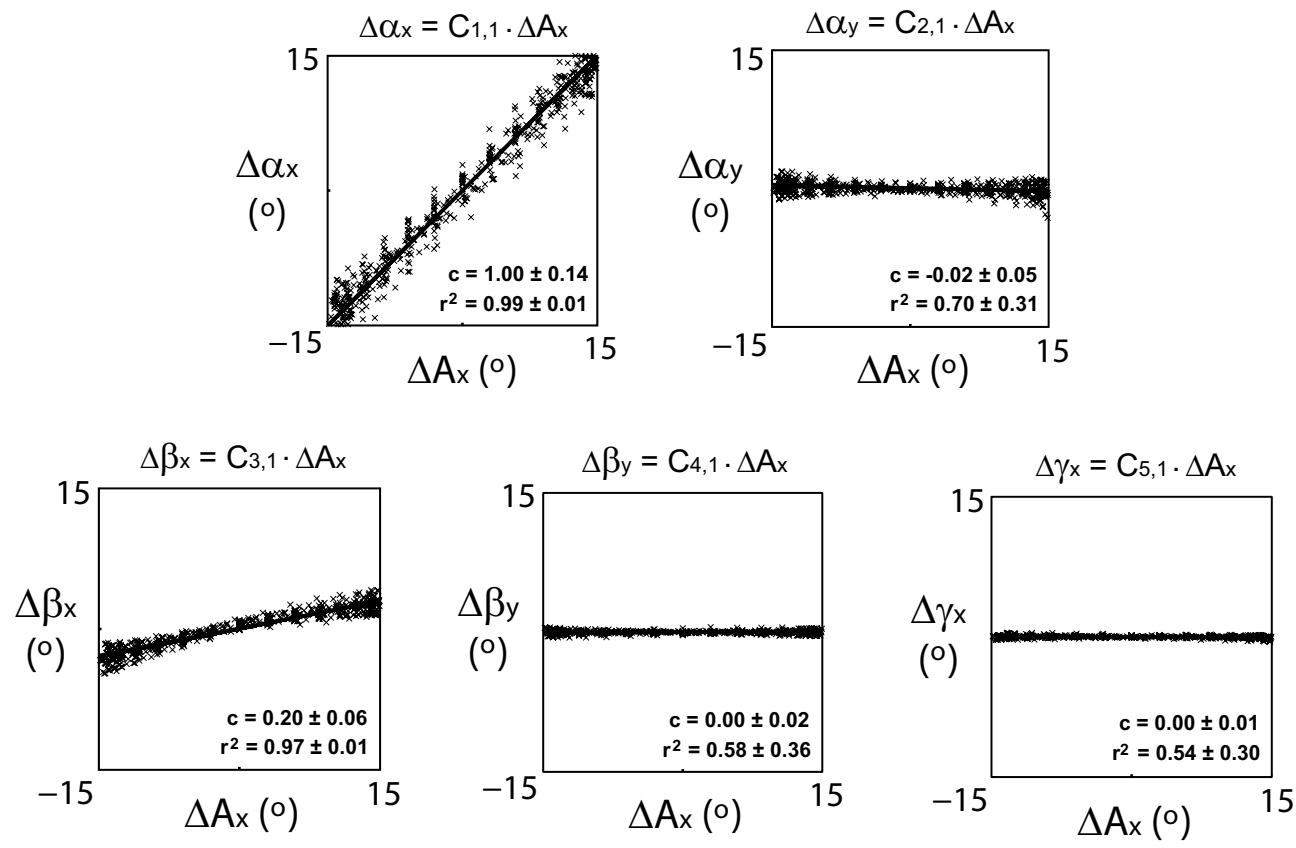

Figure 3.5: The result of the first trial which investigates the relations (linear best-fits) between sagittal backrest adjustment $\left(\Delta \mathrm{A}_{\mathrm{x}}\right)$ and all body segments rotations $\left(\Delta \alpha_{\mathrm{x}}, \Delta \alpha_{\mathrm{y}}, \Delta \beta_{\mathrm{x}}, \Delta \beta_{\mathrm{y}}, \Delta \gamma_{\mathrm{x}}\right)$. The slope coefficients (c) and coefficients of determination $\left(\mathrm{r}^{2}\right)$ are shown for all relations.

$$
q=C \cdot u
$$

in which,

$$
\mathbf{C}=\left[\begin{array}{ccccc}
\mathbf{1 . 0 0} & 0.01 & \mathbf{0 . 1 5} & -0.02 & -0.08 \\
-0.02 & \mathbf{1 . 0 7} & -0.01 & -\mathbf{0 . 1 2} & 0.01 \\
\mathbf{0 . 2 0} & -0.05 & \mathbf{0 . 5 2} & 0.01 & \mathbf{0 . 1 8} \\
0.00 & \mathbf{0 . 2 4} & \mathbf{0 . 0 1} & \mathbf{0 . 3 5} & -0.01 \\
0.00 & \mathbf{0 . 0 4} & \mathbf{0 . 0 4} & \mathbf{0 . 0 4} & \mathbf{0 . 9 7}
\end{array}\right]
$$

Figure 3.6: Matrix equation that relates angular chair configuration $(\mathrm{u})$ to body segments orientation (q). The bold values in C involve slope coefficients (c) with high coefficient of determination $\left(\mathrm{r}^{2}>0.8\right)$.

$$
u=C^{-1} \cdot q
$$

in which,

$$
\mathbf{C}^{-1}=\left[\begin{array}{ccccc}
1.06 & -0.04 & -0.31 & 0.03 & 0.15 \\
0.02 & 0.87 & 0.01 & 0.30 & 0.00 \\
-0.41 & 0.11 & 2.05 & 0.00 & -0.43 \\
0.00 & -0.60 & -0.06 & 2.65 & 0.05 \\
0.02 & -0.02 & -0.08 & -0.12 & 1.05
\end{array}\right]
$$

Figure 3.7: Matrix equation (with inverted C) that relates body segments orientation (q) to angular chair configuration (u). 
imposed from backrest angle adjustment around a pivot within the lumbar-thoracic region.

We choose to compute the coefficient matrix $C$ from the linear effects of chair segments adjustment separately. Identical results have been found when calculating $C$ in a least square sense. Nevertheless, we preferred the first approach since this provides the information about the couplings between individual chair- and body segments rotation in a way that improves interpretation of our findings.

The computed C-matrix holds the linear effects of angular chair adjustments on the alignment of the trunk, pelvis and thighs. The design for posture adjustment was based on the assumption that the axes for chair segments rotation aligned the anatomical axes for body segments rotation. This implies that the trunk, pelvis and thighs could be adjusted independently and that the C-matrix should be diagonal. However, some couplings have been found outside the diagonal. We explain this by the fact that the anatomical axes for body segments rotation do not concern pure pivots which complicates the alignment with the axes for chair adjustments. Individual chair segment adjustments do therefore not only influence the supported body segment but also have secondary effect on adjacent body segments. Secondary couplings with any significance $(\mathrm{c}>0.1)$ are found between the backrest $\left(\Delta \mathrm{A}_{\mathrm{x}}\right)$ and pelvis $\left(\Delta \beta_{\mathrm{x}}\right)$ in the sagittal plane $\left(\mathrm{C}_{3,1}=0.20\right)$, between the backrest $\left(\Delta \mathrm{A}_{\mathrm{y}}\right)$ and pelvis $\left(\Delta \beta_{\mathrm{y}}\right)$ in the frontal plane $\left(\mathrm{C}_{4,2}=0.24\right)$, between the parallelogram $\left(\Delta \mathrm{B}_{\mathrm{x}}\right)$ and trunk $\left(\Delta \alpha_{\mathrm{x}}\right)$ in the sagittal plane $\left(\mathrm{C}_{1,3}=0.15\right)$, between the seat parts $\left(\Delta \mathrm{B}_{\mathrm{y}}\right)$ and trunk $\left(\Delta \alpha_{\mathrm{y}}\right)$ in the frontal plane $\left(\mathrm{C}_{2,4}=-0.12\right)$, and between the seat $\left(\Delta \mathrm{H}_{\mathrm{x}}\right)$ and pelvis $\left(\Delta \alpha_{\mathrm{x}}\right)$ in the sagittal plane $\left(C_{3,5}=0.18\right)$. Positive correlations are found for all couplings expect for the one between $\Delta \mathrm{B}_{\mathrm{y}}$ and $\Delta \alpha_{\mathrm{y}^{*}}$. This latter indicates that the direction of the coupling between the pelvis and the trunk is different for seat part adjustments than for frontal backrest angle adjustments. In the sagittal plane, the pivot for backrest rotation follows the axis for trunk rotation when rotating the pelvis from parallelogram adjustments. In the frontal plane however, the pivot of the backrest is fixed and does not follow the axis for trunk rotation when the pelvis rotates sideways. When the anatomical axis for frontal trunk rotation moves away from the pivot for frontal backrest rotation and the upper body stays in place due to the support of the armrest, the trunk rotates opposite to the pelvis and slides relative to the backrest when adjusting the height between the seat parts. Although the algorithm compensates for angular trunk displacement, this sliding component cannot be prevented.

The diagonal of $\mathrm{C}$ shows strong direct couplings between the backrest and the trunk $\left(C_{1,1}=1.00\right.$ and $\left.C_{2,2}=1.07\right)$, and between the seat and the thighs $\left(C_{5,5}=0.97\right)$. To rotate the pelvis in the sagittal and frontal plane, less significant couplings are found $\left(\mathrm{C}_{3,3}\right.$ $=0.52$ and $\left.C_{4,4}=0.35\right)$. During sagittal pelvis rotation $\left(\Delta \beta_{\mathrm{x}}\right)$, we observed that almost half of the parallelogram adjustment $\left(\Delta \mathrm{B}_{\mathrm{x}}\right)$ contributed to lumbar spine deformation rather than pure pelvis rotation. A stronger coupling is expected when the axis for backrest rotation is aligned closer to the lumbosacral region. The relative small coupling during frontal pelvis rotation $\left(\Delta \beta_{\mathrm{y}}\right)$ is ascribed to the deformation of the seat cushion and buttock tissue when adjusting the height between the left and right seat part. Therefore, it must be known what the seat cushion characteristics and buttock tissue properties are when to control pelvis angle in the frontal plane.

Table 3.1 shows the initial body segment angles prior to each adjustment trial. Although a strict protocol was followed to position subjects within the chair, high 
standard deviations for $\alpha_{\mathrm{x} 0}, \alpha_{\mathrm{y} 0}$ and $\beta_{\mathrm{x} 0}$ indicate significant variability between subjects for the initial alignment of the trunk and pelvis. We attribute this variation to differences in morphology, inaccuracies in marker placement and variation in pelvis mold positioning. For practical reason, we attached the mold while standing, which probably caused little displacement when subjects were seated. Although this was checked after the subjects were seated, repositioning the mold after more careful inspection might solve most variability.

Table 3.1 also shows small variability between trials for the initial alignment of all body segments, which indicates good reproducibility of the initial body posture. Because body posture returns to the initial situation after each trial we assumed minimal sliding during posture adjustments. Minimal shear at the backrest interface was assumed since the backrest was mounted on a sledge. This allows frictionless trunk displacements in vertical direction to align the axis for sagittal trunk rotation with the pivot for backrest rotation. In sagittal direction we observed that the pelvis rolled over the tuberosities and that little sliding only occurred under the thighs. Because most buttock load exerts on the ischial region, we assume that sliding of the thighs introduces minimal shear at the seat interface. Rolling over the tuberosities instead of sliding also implicates less need for clinicians to reposition the individual during daily wheelchair use because the buttock does not slide forward on seat support when adjusting the configuration of the chair.

\subsection{Conclusions}

Although the simulator chair was designed to control the trunk, pelvis and thighs independently, moderate couplings have been found between body segments when modifying angular chair configuration. Nevertheless, the predictive algorithm that has been developed from experimental measurement of body posture and chair modification seems applicable to compute angular chair configuration for desired body posture when the initial body-chair configuration is known. For clinical application, future experiments must be performed on impaired individuals to validate the model in terms of accuracy. A lack of controlled trunk muscle function and problems with spasticity are issues that influence postural response from automatic chair adjustments and make clinical evaluation necessary. Future studies must also aim at the effects of sitting posture on body load associated with wheelchair discomfort. To estimate body load from chair support forces, an accurate model that relates sitting posture to chair configuration is essential. When we know how angular chair adjustments influence body segments orientation, the next step is to evaluate whether this system is also applicable to regulate body load and to prevent sitting related mobility problems in individuals who cannot functionally reposition themselves.

\subsection{Acknowledgements}

This study was partly funded by the Dutch Ministry of Economic Affairs, SenterNovem. The authors would like to thank the engineering company Demcon (Oldenzaal, the Netherlands) for developing the experimental simulator chair. 


\section{$\underline{\text { Part III }}$}

Regulation of Body Load 


\section{Chapter 4}

\section{Sagittal Chair Adjustment and Seat Reaction Load}

Published as: P. van Geffen, J. Reenalda, P.H. Veltink and H.F.J.M. Koopman, 2008, Effects of sagittal postural adjustment on seat reaction load, Journal of Biomechanics, Volume 41, Issue 10, Page 2237-45. 


\begin{abstract}
Wheelchair-users often adopt a passive sitting posture and suffer from sitting acquired pressure ulcers (PU) that mainly occur when high buttock pressures sustain for a longer period of time. Body posture directly influences seating load and proper postural change is therefore essential. When individuals cannot reposition themselves, seating systems that adjust sitting posture are needed to regulate seating load and potentially diminish the risk to develop PU. We describe a mechanism for postural adjustments and investigated the influence of seat inclination (1), pelvis rotation (2) and chair recline (3) on buttock load.

We developed an experimental simulator chair containing the concept to adjust body posture in the sagittal plane. Measurements involved quasi-static actuated chair movements in which chair configuration, buttock interface pressure and seating forces were simultaneously measured.

Significant effects with high coefficients of determination $\left(r^{2}>0.8\right)$ were found for seating force, centre of pressure and sacral load.

We conclude that a combination of independent pelvis rotation and seat inclination is effective to regulate the net buttock shear force and the sacral interface pressure in healthy subjects. Whether both techniques are also applicable in subjects without postural stability is still to be evaluated.
\end{abstract}




\subsection{Introduction}

Wheelchair-users with limited trunk muscle function often adopt a static sitting posture and develop sitting related mobility problems including pressure ulcers (PU) (Swain and Bader, 2002, Bouten et al., 2003). Although the physiological process prior to PU remains unclear, it is accepted with some certainty that tissue breakdown mainly occurs when high pressures sustain for a longer period of time and that successive durations of pressure relief enables buttock tissue to recover from deformation and blood flow stagnation. Current beliefs discriminate between deep and superficial PU (Quintavalle et al., 2006) in which deep ulcers occur from sustained internal buttock pressure and are often located in the muscle tissue directly under bony prominences. Superficial ulcers on the other hand, occur in the skin from sustained shear force and pressure on the buttock-seat interface.

Common applications to prevent PU are based on seating surfaces that distribute the buttock pressure over a larger area and locally reduce the applied pressure under the sacrum and ischial tuberosities (Whittemore, 1998). Although the overall buttock pressure will reduce significantly, it is unlikely that the ischial pressure becomes low enough to prevent tissue perfusion from stagnating.

Rather than only focusing on peak pressure reduction, it is widely recommended to induce significant durations of pressure relief when subjected to prolonged wheelchair sitting. Most suggested movement types to relieve pressure are lifting the buttock from pushing the armrests, frontal pelvis rotations to relieve buttock pressure from side to side, and to lean forward causing the ischial region to come clear (Stockton and Rithalia, 2007). All tasks require adequate trunk muscle function and are for many wheelchair-users not applicable.

For persons who cannot reposition themselves, the industry obtained a standard that stimulates them to perform a 60 degree chair recline (tilt-in-space) once in a while. Reclining the chair up to 60 degrees is suggested enough to unload the buttock for sufficient tissue recovery (Henderson et al., 1994). Although this application seems very effective when used adequately, reclining the chair that far is considered undesirable since it interrupts during activities in daily life and in some cases, it even effects breathing and muscle spasms.

When subjects cannot preposition themselves and static cushions do not relieve buttock pressure enough, dynamic systems that adjusts the seating surface are effective to redistribute buttock pressure and are considered useful within proper pressure management (Makhsous et al., 2007, Stockton and Rithalia, 2007). However, it is emphasized that it is very unwise to apply dynamic cushions and completely replace the need for postural movements, especially when passive sitting behavior is also associated with mobility problems other than PU.

The inability to reposition implies that adequate postural change is only possible when adjusting chair configuration. Because pelvic alignment is crucial for the adopted posture (Janssen-Potten et al., 2001, Koo et al., 1996, Lucas et al., 2005, Maltais et al., 1999, Sprigle et al., 2003) and buttock load (Maltais et al., 1999, Hobson and Tooms, 1992, Koo et al., 1996, Tam et al., 2003), systems that control pelvis angle relative to the seat could play a key role in proper pressure management. With this purpose, we developed an experimental simulator chair that independently controlled the orientation of the trunk, pelvis and thighs. We describe a mechanical 
concept for postural change and we investigate the influence on seating load alteration. We compare the effects of seat inclination relative to the pelvis and trunk (1), pelvis rotation relative to the seat and trunk (2) and chair recline relative to the line of gravity (3). It is our goal to investigate which approach is most effective to redistribute buttock load.

\subsection{Methods}

\subsubsection{Subjects}

Eighteen healthy male subjects (age $22 \pm 2$ years, weight $75 \pm 8 \mathrm{~kg}$, Length $1.84 \pm$ $0.05 \mathrm{~m}$, BMI $22.8 \pm 1.94 \mathrm{~kg} / \mathrm{m}^{2}$ ) were recruited for this study. Prior to the experiment, all subjects read and signed and 'informed consent' in which the objective and experimental protocol was explained. This study was approved by the Committee for Medical Ethics of Roessingh Rehabilitation Centre (Enschede, the Netherlands).

\subsubsection{Concept for postural adjustments}

To investigate the effects of postural adjustments, a simulator chair was developed that adjusts sitting posture in the sagittal plane. Figure 4.1 shows a mechanical concept for independent body segment actuation.
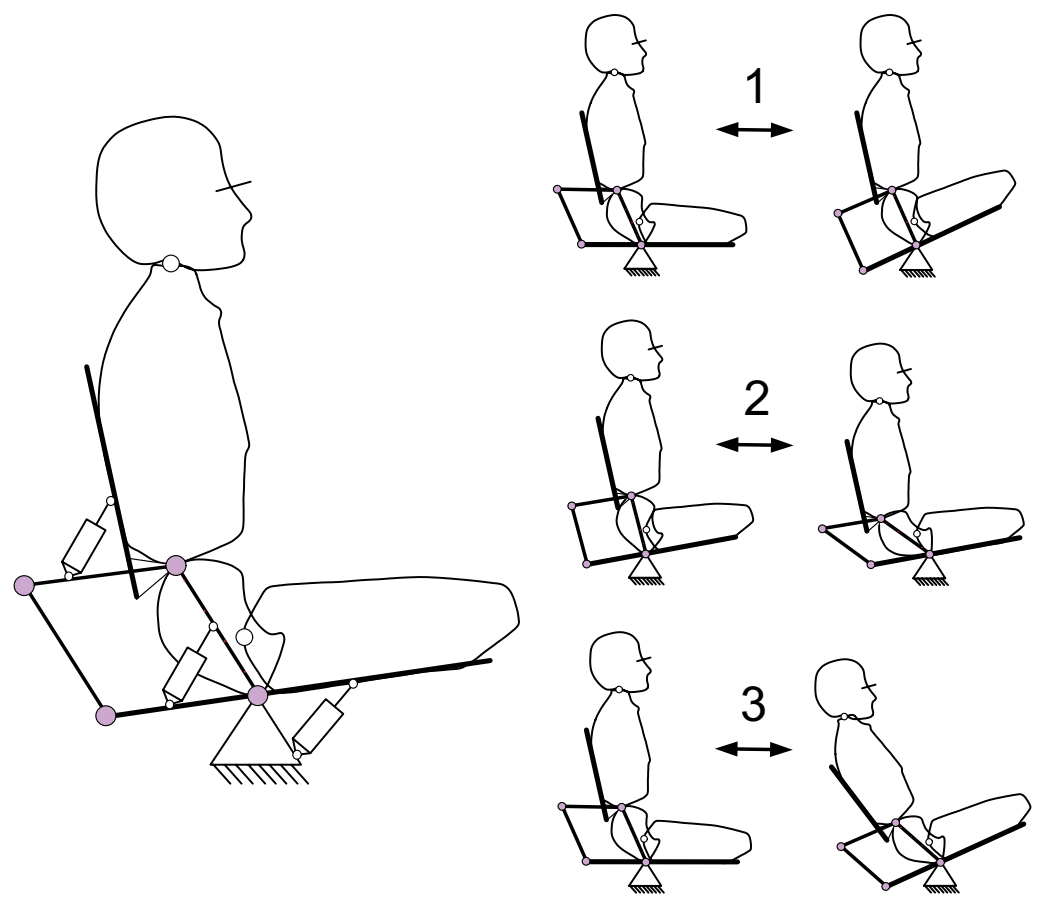

Figure 4.1: Mechanical concept for postural adjustment. Situations of sagittal seat inclination (1), pelvis rotation (2) and chair recline (3) are shown. 
This concept was based on a parallelogram on which the backrest was fixed. The upper body was supported above the lumbar spine, the pivot point of the backrest was placed within the lumbar region and the pivot point of the seat was located under the tuberosities. Aligning the pelvis with the parallelogram makes independent pelvis rotation possible. The backrest was actuated from a parallelogram that has its base on the back part of the seat. The configuration of the parallelogram imposes pelvis rotation and was actuated from the back seat. The whole mechanism rotated around a pivot located under the tuberosities and was externally actuated. Figure 4.1 also presents situations of actuated seat inclination relative to the pelvis and trunk (1), pelvis rotation relative to the seat and trunk (2) and chair recline relative to the line of gravity (3).

\subsubsection{Experimental setup}

Experiments were done with an instrumented simulator chair (figure 4.2) containing the mechanical concept for postural adjustments. The backrest was mounted on a sledge to allow up- and downward translations when the pelvis was not properly aligned. A counter weight was used to support the weight of the backrest. The depth and height of the backrest, seat and leg rests were individually adjustable. The distance between the seat and back could be changed by adjusting the height of the parallelogram.

For accurate 3D seating force measurements, six uni-axial force sensors (Tension \& Compression Load Cell, FUTEK, California, USA) were mounted in the seat frame. The operating system for chair actuation and real-time data processing was based on a Matlab/Simulink environment. 3D chair configuration was obtained using a six infrared camera VICON motion capturing system. Three reflective markers were placed on the backrest, four on the seat and four on the pivot points of the parallelogram.

A Tekscan Comfortmat pressure mapping device (Teksan inc, Boston Massachusetts, USA) was placed over the seat and was used to measure pressure distribution. The device consists of 1024 (32 x 32) rectangular pressure elements of $15 \mathrm{~mm}$ x $15 \mathrm{~mm}$ and was calibrated with an upper limit of $33 \mathrm{kPa}(250 \mathrm{mmHg})$.

\subsubsection{Data analyses}

To relate chair kinematics, pressure distribution and seating force, a global reference frame $(G)$ was defined in which the $x-y$ plane was aligned with the anatomical transverse plane, the $y-z$ plane with the anatomical sagittal plane and the $\mathrm{x}-\mathrm{z}$ plane with the anatomical frontal plane of the seated subject (figure 4.2). Local coordinate frames for the seat $\left(\mathrm{R}_{\mathrm{s}}\right)$, backrest $\left(\mathrm{R}_{\mathrm{b}}\right)$ and parallelogram $\left(\mathrm{R}_{\mathrm{p}}\right)$ were constructed and used to define the exact configuration of the chair $(\phi, \beta$ and $\alpha)$. We used the analogue force data to calculate the seat reaction force and centre of pressure (cp) relative to the seat. Shear- $\left(\mathrm{F}_{\mathrm{s}}\right)$ and the normal force $\left(\mathrm{F}_{\mathrm{n}}\right)$ were defined parallel and perpendicular to the seat plane. Buttock interface pressure distribution was derived from the analogue pressure data (Fig 4.3). Tuberal and sacral load $\left(\mathrm{P}_{\mathrm{t}}\right.$ and $\mathrm{P}_{\mathrm{s}}$ ) defined the average pressure as measured within square areas $(60 \mathrm{~mm} \times 60 \mathrm{~mm})$ under the tuberosities and sacrum respectively. A multiple comparison one-way ANOVA was performed to determine 
which quantities (cp, $\mathrm{P}_{\mathrm{t}}, \mathrm{P}_{\mathrm{s}}, \mathrm{F}_{\mathrm{n}}$ and $\mathrm{F}_{\mathrm{s}}$ ) were significantly different between methods (seat inclination, pelvis rotation and chair recline).

\subsubsection{Experimental protocol}

Prior to the experiment an empty seated force calibration measurement was done. Subjects were seated with their tuberosities aligned with the seat pivot point. Pressure mapping was used to position the tuberosities. Reflective markers were placed on the seat, backrest and parallelogram. The seat and footrests were adjusted for optimal pressure distribution. The thighs were fully supported except for the back of the knee. The footrests were set perpendicular to the seat and only the weights of the lower legs were supported. The latter was estimated from the gravitational force component on the seat which we monitored during footrest adjustment. The height of the parallelogram and depth of the backrest was individually adjusted to centre the pivot point of the backrest within the lumbar region at a level of the third lumbar vertebrae. Within the initial chair configuration, the seat plane $(\phi)$ was inclined 13 degrees. The parallelogram $(\alpha)$ and backrest $(\beta)$ angles were set respectively to 25 and 15 degrees relative to the seat.

Subjects were asked to adopt a passive sitting posture and to prevent redundant trunk muscle function. A static reference trial was performed to define the tuberal and sacral areas from which the average interface pressures were calculated.

From the initial chair configuration we imposed seat inclination, pelvis rotation and chair recline by respectively actuation the seat, parallelogram and the whole chair in the sagittal plane. Measurements involved 2 cycles of quasi-static actuated movements within a range of 25 degrees and a time period of 30 seconds. Two pre-measurement

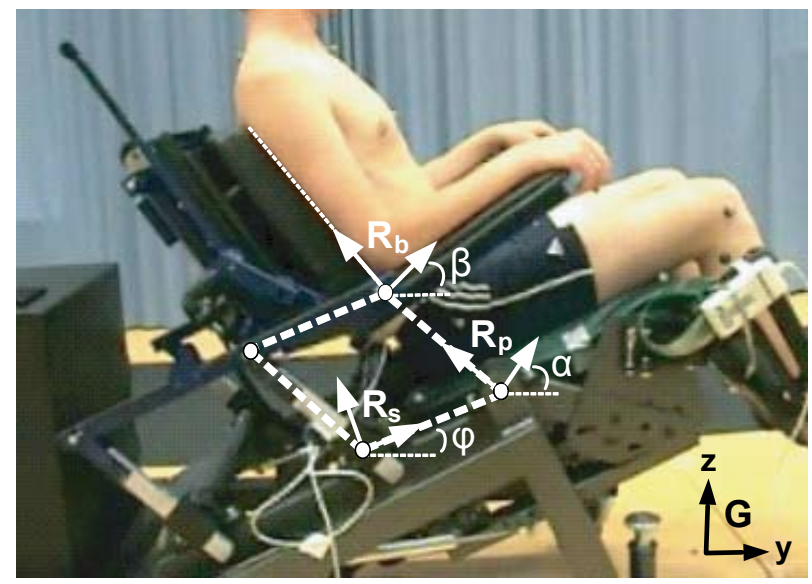

Figure 4.2: Experimental setup. Adjustable instrumented simulator chair containing the mechanical concept for postural adjustments. Six uni-axial force sensors were mounted in the seat frame and a pressure mapping device was used to measure pressure distribution. 3D chair configuration was obtained using a six-camera VICON motion capturing system. Local coordinate frames $\left(R_{s}, R_{b}\right.$ and $\left.R_{p}\right)$ were constructed relative to $G$ and chair configurations $(\alpha, \beta$ and $\phi$ ) were defined. 

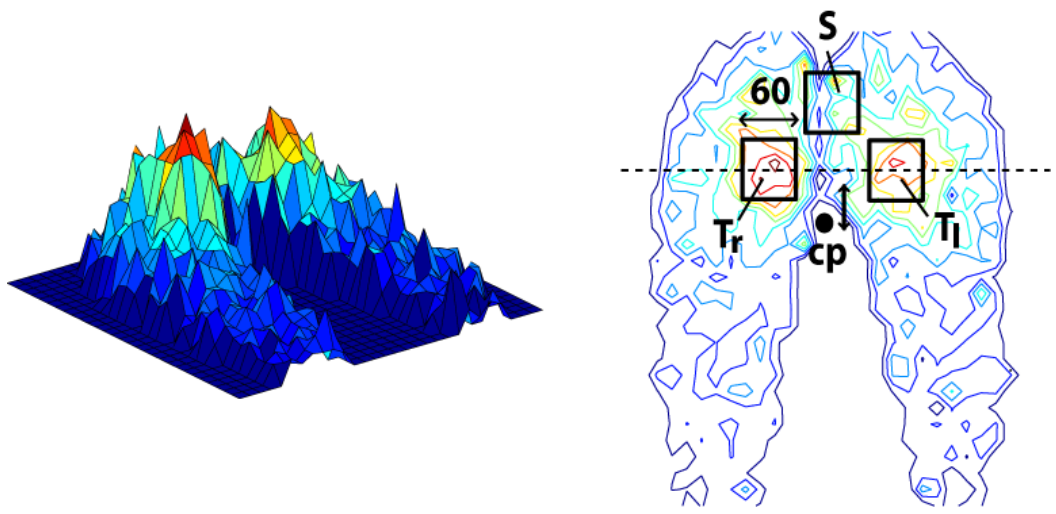

Figure 4.3: Buttock interface pressure distribution. Surface- and contour plots are shown of the buttock interface pressure. High pressures are shown in the tuberal and sacral region. $60 \mathrm{~mm}$ x 60mm square surfaces defined the tuberal $\left(T_{r}\right.$ and $\left.T_{1}\right)$ and sacral $(S)$ areas. The dashed line represents tuberal location and the axis of rotation for both the pelvis and seat plane. Centre of pressure (cp) displacement is defined relative to this axis (dashed line).

movement cycles were performed to familiarize chair adjustments. During each trail, 3D marker position, seating force and seating pressure were recorded for offline analysis.

\subsection{Results}

Initial values and ranges $(\Delta)$ for $\mathrm{cp}, \mathrm{F}_{\mathrm{s}}, \mathrm{F}_{\mathrm{n}, \mathrm{P}} \mathrm{P}_{\mathrm{t}}$ and $\mathrm{P}_{\mathrm{s}}$ during chair adjustments are shown in table 4.1. All quantities $\left(\Delta \mathrm{cp}, \Delta \mathrm{F}_{\mathrm{s}}, \Delta \mathrm{F}_{\mathrm{n}}, \Delta \mathrm{P}_{\mathrm{s}}\right.$ and $\left.\Delta \mathrm{P}_{\mathrm{t}}\right)$ are expressed as percentage of its total range (\%range) and related to seat inclination, pelvis rotation and chair recline. Note that the number of trials is different between methods because for some trials we encountered problems with marker visibility. For seat inclination, high significant $(\mathrm{p}<0.05)$ coefficients of determination $\left(\mathrm{r}^{2}>0.8\right)$ were found for $\Delta \mathrm{F}_{\mathrm{s}}$ and $\Delta \mathrm{P}_{\mathrm{s}}$ (figure 4.4). No significant relations were found for $\Delta \mathrm{cp}, \Delta \mathrm{F}_{\mathrm{n}}, \Delta \mathrm{P}_{\mathrm{t}}$. For pelvis rotation (figure 4.5) we only found high coefficients of determination for $\Delta \mathrm{cp}$ and $\Delta \mathrm{P}_{\mathrm{s}}$. The influence of chair recline is shown in figure 4.6. For this method, strong significant relations were found for $\Delta \mathrm{cp}, \Delta \mathrm{F}_{\mathrm{s}}$ and $\Delta \mathrm{F}_{\mathrm{n}}$.

\subsubsection{Multiple comparisons}

We grouped $\Delta \mathrm{cp}, \Delta \mathrm{F}_{\mathrm{s}}, \Delta \mathrm{F}_{\mathrm{n}}, \Delta \mathrm{P}_{\mathrm{s}}$ and $\Delta \mathrm{P}_{\mathrm{t}}$ in bar diagrams (figure 4.7) to compare between methods: seat inclination (1), pelvis rotation (2) and chair recline (3). A multiple comparison one-way ANOVA was performed to determine which of these quantities were significantly $(\mathrm{p}<0.05)$ different. Figure 4.7 shows that the variation in shear force $\left(\Delta \mathrm{F}_{\mathrm{s}}\right)$ is significantly larger during seat inclination. For the variation in normal force $\left(\Delta \mathrm{F}_{\mathrm{n}}\right)$, the influence of chair recline is significantly largest. To influence the interface pressure applied under the sacrum and the tuberosities $\left(\Delta \mathrm{P}_{\mathrm{s}}\right.$ and $\left.\Delta \mathrm{P}_{\mathrm{t}}\right)$, chair recline seems least effective. For centre of pressure displacements $(\Delta \mathrm{cp})$, the largest effect is observed during pelvis rotations. 
Table 4.1: Centre of pressure, contact forces and interface pressures for the initial chair configuration and during chair adjustments.

\begin{tabular}{|c|c|c|c|c|c|c|c|}
\hline & \multicolumn{2}{|c|}{ Seat inclination $(\mathrm{n}=18)$} & \multicolumn{2}{|c|}{ Pelvis rotation $(\mathrm{n}=17)$} & \multicolumn{2}{|c|}{ Chair recline $(\mathrm{n}=15)$} \\
\hline & & Initial & Range $(\Delta)$ & Initial & Range $(\Delta)$ & Initial & Range $(\Delta)$ \\
\hline ср & {$[\mathrm{mm}]$} & $51 \pm 19$ & $15 \pm 7$ & $53 \pm 18$ & $38 \pm 6$ & $54 \pm 20$ & $19 \pm 3$ \\
\hline $\mathrm{F}_{\mathrm{s}}$ & {$[\mathrm{N} / \mathrm{kg}]$} & $-0.01 \pm 0.22$ & $1.25 \pm 0.22$ & $-0.02 \pm 0.34$ & $0.49 \pm 0.20$ & $-0.08 \pm 0.18$ & $1.04 \pm 0.08$ \\
\hline $\mathrm{F}_{\mathrm{n}}$ & {$[\mathrm{N} / \mathrm{kg}]$} & $7.00 \pm 0.41$ & $0.50 \pm 0.23$ & $7.09 \pm 0.51$ & $0.43 \pm 0.13$ & $6.78 \pm 0.27$ & $0.74 \pm 0.06$ \\
\hline$P_{t}$ & {$[\mathrm{~Pa} / \mathrm{kg}]$} & $182 \pm 22$ & $27 \pm 13$ & $189 \pm 21$ & $20 \pm 8$ & $189 \pm 24$ & $10 \pm 5$ \\
\hline $\mathrm{P}_{\mathrm{s}}$ & {$[\mathrm{Pa} / \mathrm{kg}]$} & $131 \pm 27$ & $70 \pm 20$ & $123 \pm 24$ & $69 \pm 20$ & $144 \pm 28$ & $10 \pm 3$ \\
\hline
\end{tabular}

\subsection{Discussion}

The goal of this study was to describe a concept for sagittal postural adjustments and to investigate the efficacy of chair configuration on buttock load redistribution. We found that seat inclination was most effective to regulated buttock shear force $\left(\mathrm{F}_{\mathrm{s}}\right)$ and that pelvis rotation was most effective to regulate centre of pressure $(\mathrm{cp})$ and sacral load $\left(\mathrm{P}_{\mathrm{s}}\right)$. Because pelvis angle and seat inclination could be controlled independently, no confliction coupling between $\mathrm{P}_{\mathrm{s}}$ and $\mathrm{F}_{\mathrm{s}}$ were expected.

To influence seating force, we found that seat inclination was effective to regulate $F_{s}$ and that chair recline was effective to regulate normal force $\left(F_{n}\right)$. For the sacral and tuberal interface pressures $\left(\mathrm{P}_{\mathrm{s}}\right.$ and $\left.\mathrm{P}_{\mathrm{t}}\right)$ we found that the influence of chair recline was least effective. Constant interface pressures together with a decrease in $\mathrm{F}_{\mathrm{n}}$ means that chair recline mainly affects the weight bearing component of the thighs rather than decreasing $\mathrm{P}_{t}$ and $\mathrm{P}_{\mathrm{s}}$. We found great influence on $\mathrm{P}_{\mathrm{s}}$ for both seat inclination and pelvis rotation. Both latter methods caused the sacrum to loose contact almost completely and were effective to change $\mathrm{P}_{\mathrm{s}}$ up to $70 \%$ from its initial value. To displace centre of pressure (cp) and thereby shift the net contact load to other tissue regions, the latter method seemed most effective. High coefficients of determination indicate that $\mathrm{cp}$ and $\mathrm{P}_{\mathrm{s}}$ could both be regulated from pelvis rotations. Although the effect on $\mathrm{P}_{t}$ was most significant when adjusting the seat or parallelogram, we observed that $\mathrm{P}_{\mathrm{t}}$ only changed $12 \%$ from its initial value.This relatively small influence was ascribed to the dimensions of the tuberal area. When adjusting the angle between the seat and parallelogram only 25 degrees, the ischial tuberosities mainly rolled within these areas causing that only minimal pressure change was measured. Adjusting the parallelogram within a wider range of motion might affect $\mathrm{P}_{\mathrm{t}}$ more significantly. For a more substantial effect on $\mathrm{P}_{\mathrm{t}}$, we consider investigating the possibility to adjust the pelvis in the frontal plane which is suggested being an effective technique to alternately relieve tuberal pressure from side to side (Stockton and Rithalia, 2007). In the sagittal plane, automatic chair recline and leaning forward are considered effective movement techniques to relieve buttock load once in a while (Henderson et al., 1994, Koo et al., 1996, Sprigle et al., 2007). It is reported that ischial peak pressures 

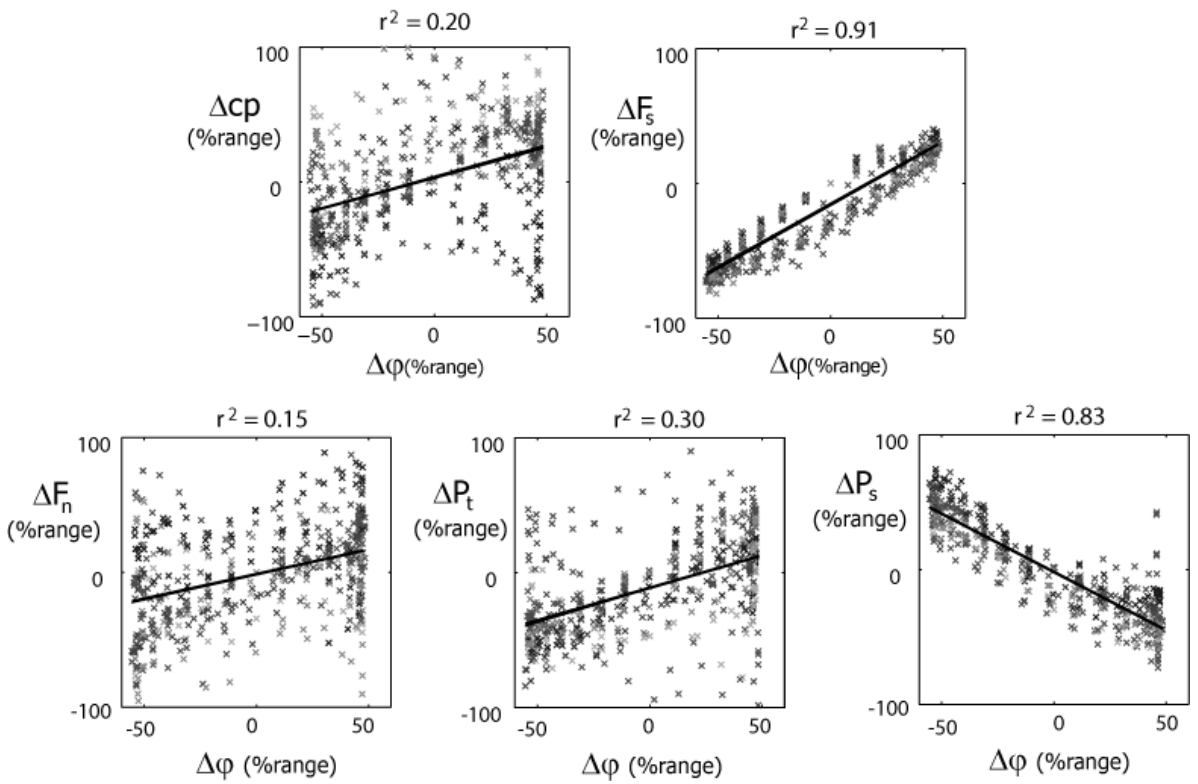

Figure 4.4: Effects of seat inclination $(\Delta \phi)$ on centre of pressure displacement $(\Delta \mathrm{cp})$ and seating load alteration $\left(\Delta \mathrm{F}_{\mathrm{s}}, \Delta \mathrm{F}_{\mathrm{n}}, \Delta \mathrm{P}_{\mathrm{t}}\right.$ and $\left.\Delta \mathrm{P}_{\mathrm{s}}\right)$ expressed as percentage of its total range (\% range). High significant $(\mathrm{p}<0.05)$ coefficients of determination $\left(\mathrm{r}^{2}>0.8\right)$ are shown for $\Delta \mathrm{F}_{\mathrm{s}}$ and $\Delta \mathrm{P}_{\mathrm{s}}$.
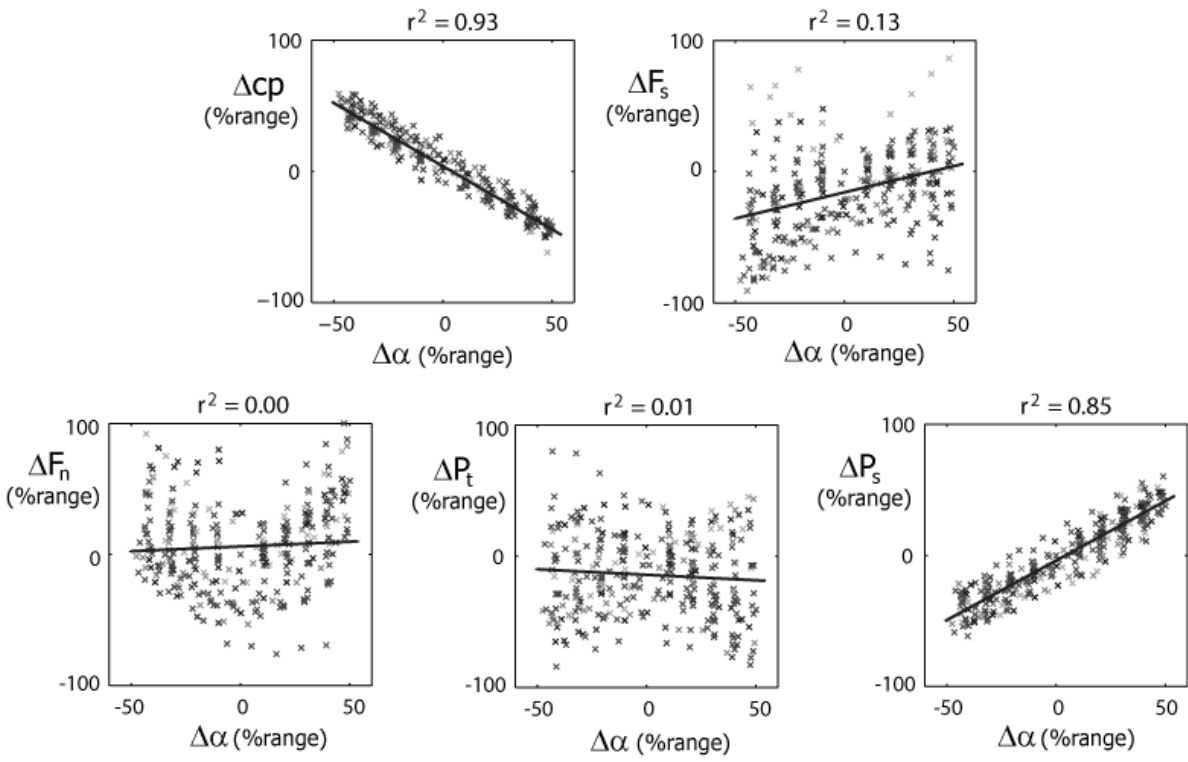

Figure 4.5: Effects of actuated pelvis rotation $(\Delta \alpha)$ on centre of pressure displacement $(\Delta \mathrm{cp})$ and seating load alteration $\left(\Delta \mathrm{F}_{\mathrm{s}}, \Delta \mathrm{F}_{\mathrm{n}}, \Delta \mathrm{P}_{\mathrm{t}}\right.$ and $\left.\Delta \mathrm{P}_{\mathrm{s}}\right)$ expressed as percentage of its total range (\%range). High significant ( $\mathrm{p}<0.05)$ coefficients of determination $\left(\mathrm{r}^{2}>0.8\right)$ are shown for $\Delta \mathrm{cp}$ and $\Delta \mathrm{P}_{\mathrm{s}}$. 

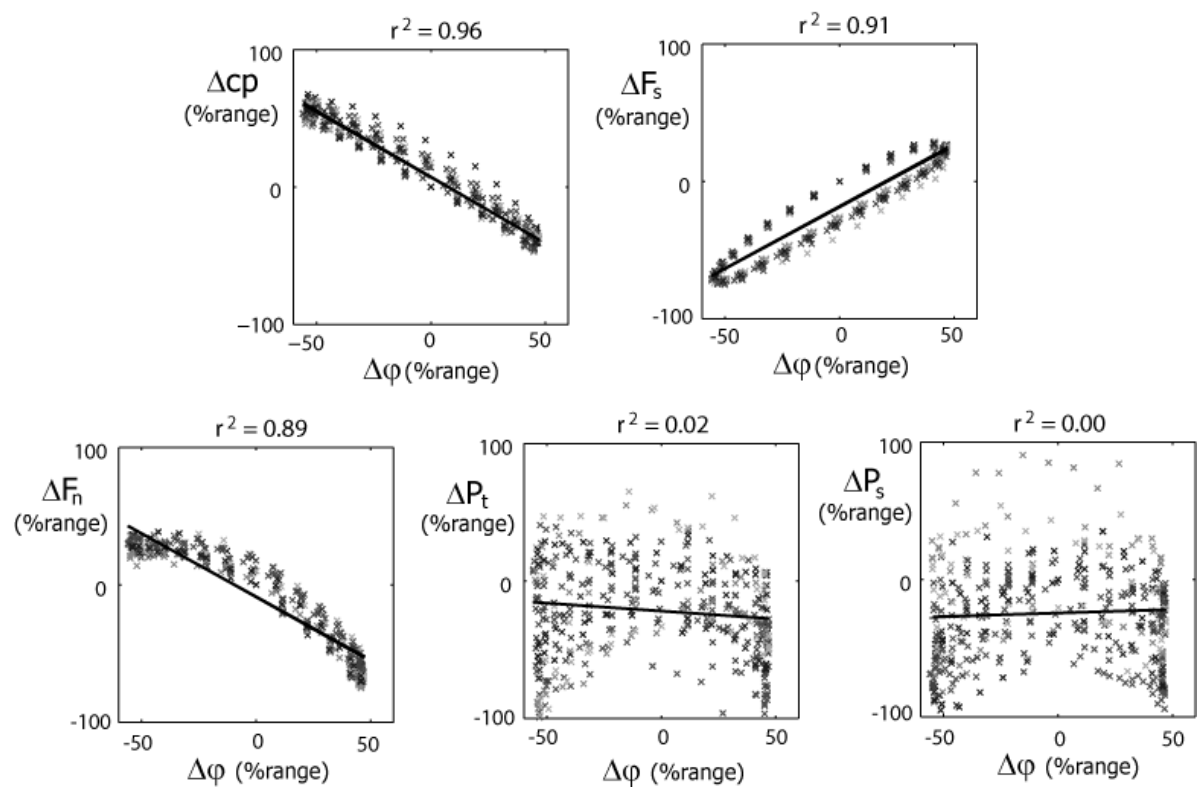

Figure 4.6: Effects of chair recline $(\Delta \phi)$ on centre of pressure displacement $(\Delta \mathrm{cp})$ and seating load alteration $\left(\Delta \mathrm{F}_{\mathrm{s}}, \Delta \mathrm{F}_{\mathrm{n}}, \Delta \mathrm{P}_{\mathrm{t}}\right.$ and $\left.\Delta \mathrm{P}_{\mathrm{s}}\right)$ expressed as percentage of its total range (\% range). High significant $(\mathrm{p}<0.05)$ coefficients of determination $\left(\mathrm{r}^{2}>0.8\right)$ are shown for $\Delta \mathrm{cp}, \Delta \mathrm{F}_{\mathrm{s}}$ and $\Delta \mathrm{F}_{\mathrm{n}}$.

relieve $32 \%$, $40 \%$ and $47 \%$ when reclining the chair $35^{\circ}, 50^{\circ}$ and $65^{\circ}$ respectively. Dependent of the level of forward leaning, ischial peak pressures relieve up $40 \%$ when leaning forward $45^{\circ}$ and even up to $80 \%$ when leaning forward completely. This latter method requires adequate trunk muscle function and is therefore not applicable for many impaired individuals. Makhsous (Makhsous et al., 2003, Makhsous et al., 2007) evaluated the effects of an adjustable ischial support on the buttock interface pressure. When tilting the ischial support downward, the buttock pressure under the ischial region relieved $40 \%$ and shifted to the thighs and the lower back. Because buttock contact pressures provide only little information about the internal mechanical tissue conditions (Oomens et al., 2003), Lim evaluated whether adjusting the ischial support also influenced the internal buttock tissue by using a 3D finite element buttock model (Lim et al., 2007). They reported that adjusting the ischial support not only relieved pressure on the superficial buttock tissue but also induced significant effects in reducing the mechanical stress inside the buttock tissue which they associated with a reduced risk to develop deep tissue breakdown. In some way, the concept introduced by Makhsous shows some similarities with the device described in our study. Similar to the mechanical effects when tilting the ischial support downward, anterior pelvis rotations relieves buttock load under the sacrum and shifted towards to the thighs and lower back. The latter was only assumed since no low back pressure measurements were obtained. A drawback however, is that the pelvis rolls over the ischial tuberosities which makes that significant pelvis rotations are necessary to benefit the sub-tuberal tissue structures from the induced pressure 
variation. The advantage on the other hand is that pressure variations are induced from adjusting body posture. Where adjustable seat surfaces regulate buttock load without affecting body posture, we strived to regulate buttock load from adjusting body posture which is positively associated with sitting related mobility problems other than PU.

The variability for the buttock interface pressures as shown in table 4.1 seemed relatively large. Because these variations did not significantly influence the relations we found between chair configuration and pressure redistribution, we expect no effects concerning the main outcome of this study. The variability between subjects were considered normal compared to studies (Kernozek and Lewin, 1998, Kernozek et al., 2002) where they investigated the effects of body build on peak pressure distribution. Kernozek (Kernozek et al., 2002) reported inter-individual variation up to $30 \%$ between thin (BMI $<20 \mathrm{~kg} / \mathrm{m}^{2}$ ) and 'normal' (BMI $20-25 \mathrm{~kg} / \mathrm{m}^{2}$ ) subjects and might explain the variation showed within our study. For the intra-individual variability, we only found relatively large variations for the sacral interface pressure. Because pelvis alignment seemed strongly related to the level of sacral pressure, we address this variability to differences in the initial pelvis positioning.

For optimal buttock load regulation, the unavoidable questions arise how long the buttock tissue can sustain certain pressure levels before tissue degeneration takes place and how long it takes to recover the buttock tissue from prolonged deformation. To associate tissue breakdown to pressure and time, much trouble has been taken to identify reliable thresholds for minimal pressure and duration which
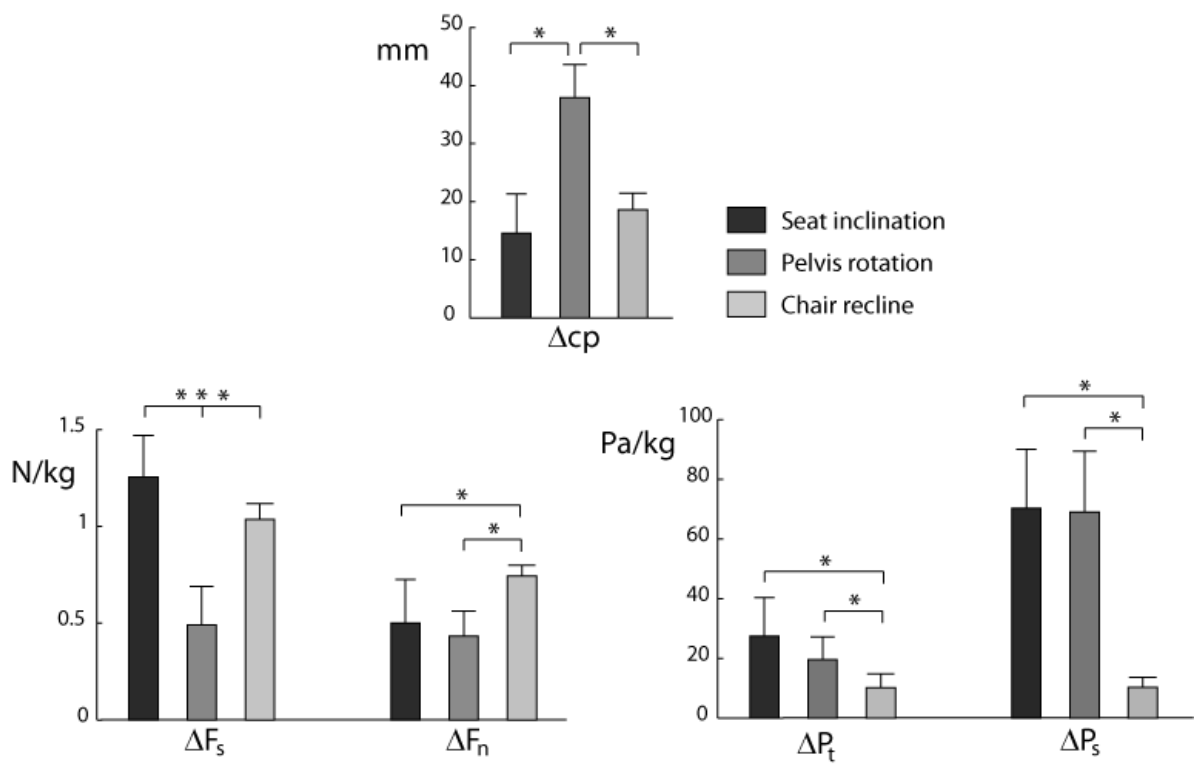

Figure 4.7: Multiple comparison diagrams. The effect of seat inclination, pelvis rotation and chair recline on the variation in centre of pressure $(\Delta \mathrm{cp})$, seating force $\left(\Delta \mathrm{F}_{\mathrm{s}}\right.$ and $\left.\Delta \mathrm{F}_{\mathrm{n}}\right)$ and the interface pressure applied under the sacrum and the tuberosities $\left(\Delta \mathrm{P}_{\mathrm{s}}\right.$ and $\left.\Delta \mathrm{P}_{\mathrm{t}}^{\mathrm{s}}\right)$. *Significant $(\mathrm{p}<$ $0.05)$ difference between methods. *** Significant $(\mathrm{p}<0.05)$ difference between all methods. 
consistently generates tissue necrosis (Bouten et al., 2003, Linder-Ganz et al., 2006). However, no reliable indicator for PU risk is yet applicable. The US Department of Health recommends wheelchair-users to relieve pressure every hour and to shift body weight every 15 minutes (Agency for Health Care Policy and Research, 1994). Whether this also applies for our concept is still something to evaluate.

We are aware that the lack of controlled trunk muscle function, buttock (muscle) tissue atrophy and problems with spasticity are issues that influence postural response from chair adjustments. Because we only evaluated the effects of chair adjustments on buttock load in healthy subjects it is still to be evaluated how these findings translate to impaired persons.

Besides a positive contribution on pressure management, adjusting the pelvis without affecting the trunk also influences the mechanical conditions of the lumbar spine which is associated with all kinds of low back disorders (Verver et al., 2003, Lengsfeld et al., 2000a, Lengsfeld et al., 2000b, McGregor et al., 1997). When sitting postures with poor pelvis alignment are maintained for a longer period of time, sustained mechanical tissue loading of the lumbar spine causes the inter-vertebral discs to degenerate which are assumed as an important cause for low back pain. The concept for independent pelvis rotations directly influences the curvature of the lumbar spine and can be used to positively regulate lumbar load.

\subsection{Conclusions}

We conclude that a combination of independent pelvis rotation and seat inclination is effective to regulate the net buttock shear force and the sacral interface pressure in healthy subjects. However, we were not satisfied with the capacity to regulate the pressures applied on the ischial tuberosities. We therefore consider investigating the possibility to adjust the pelvis in the frontal plane. To evaluate the effect of pelvis rotation more closely, future studies must also aim at the influence of anatomical pelvis alignment (both in the sagittal- and frontal plane) rather than only focusing on the configuration of the chair. The influence of a larger range of pelvis motion and the effects on lumbar mobility and lumbar spine loading are also interesting issues to investigate. Although this study was performed on healthy subjects, our findings suggest that it might be interesting to apply the described concept for buttock load redistribution into clinical practice.

\subsection{Acknowledgements}

This study was partly funded by the Dutch Ministry of Economic Affairs, SenterNovem. We would like to thank the engineering company Demcon (Oldenzaal, the Netherlands) for developing of the experimental simulator chair. We also thank Bert Faber (Welzorg Special Products, Oldenzaal, the Netherlands) for sharing his expertise in seating ergonomics. 


\section{Chapter 5}

\section{Decoupled Pelvis Rotations to Regulate Buttock Load}

Published as: P. van Geffen, J. Reenalda, P.H. Veltink and H.F.J.M. Koopman, 2009, Decoupled pelvis rotation in sitting: A passive motion technique that regulates buttock load associated with pressure ulcer development. Journal of Biomechanics (in press) 


\begin{abstract}
Wheelchair-users who cannot reposition themselves often suffer from pressure ulcers which are places of tissue breakdown in the buttock region under the sacrum and ischial tuberosities. Periodic pressure relief is needed to recover the buttock tissue from continuous deformation and impairment of tissue perfusion. Because pelvis alignment directly affects body posture and buttock load, a passive motion technique was developed that adjusts pelvis orientation independent from the trunk and seat support. This study investigates the effects of so-called decoupled pelvis rotation (DPR) on the loads at the buttock-seat interface and evaluates whether this technique is applicable to regulate buttock load in sitting.

Eighteen healthy male subjects participated in this study. Experiments were performed with a computer-aided adjustable simulator chair, instrumented with a concept of DPR. Measurements involved quasi-static actuated pelvis movements in the sagittal and frontal plane. Pelvis orientation, buttock interface pressures, seat reaction forces and centre of pressure were simultaneously measured.

As a results of the induced passive pelvis movements, the pelvis rotated $19 \pm 2^{\circ}$ and $9 \pm 2^{\circ}$ in the sagittal and frontal plane respectively. Significant relations were found between pelvis rotation and most quantities of buttock load.

Findings suggest that DPR is an effective technique to regulate buttock load in ablebodied individuals. For clinical application, this technique is still to be evaluated on individuals who cannot functionally reposition themselves. Impairments to the neuromuscular function influence postural response from chair adjustments which makes clinical investigation necessary.
\end{abstract}




\subsection{Introduction}

Wheelchair-users with reduced postural stability often adopt a 'slouched' static sitting posture during activities of daily life (Hobson and Tooms, 1992). The inability to change body posture causes continuous buttock loading, which potentially initiates the irreversible process for pressure ulcers in the tissue under the sacrum and ischial tuberosities (Bouten et al., 2003). The severity of tissue injury varies from superficial skin irritation to extensive destruction/necrosis of deeper tissue layers (Black et al., 2007, Defloor and Schoonhoven, 2004, Defloor et al., 2005). It has been reported (Bouten et al., 2003) that superficial injury develops in the skin mainly from friction/ shear at the buttock-seat interface, and that deep injury develops in muscle tissue from sustained deformation and impaired tissue perfusion. This latter form of tissue jury is a severe pressure ulcer of which the onset is hard to detect since it initiates in deeper muscle layers. It progresses outwards to the skin and when it manifests at the buttock surface it already caused severe damage to the sub-dermal tissue. This type of pressure ulcer is extremely hard to treat and sometimes results in surgery with serious consequences for someone's well-being.

Extensive studies have been performed to determine the cause, onset, location and extent of tissue damage from sustained internal tissue loading. Evidence from experimental research with muscle-cell cultures (Gawlitta et al., 2007, Gefen et al., 2008a, Gawlitta et al., 2008, Gefen et al., 2008b), finite element models (Linder-Ganz et al., 2007b, Brosh and Arcan, 2000, Ragan et al., 2002, Todd and Thacker, 1994, Lim et al., 2007, Makhsous et al., 2007a), animals (Linder-Ganz et al., 2006, Stekelenburg et al., 2006) and humans (Ohura et al., 2007, Quintavalle et al., 2006) have demonstrated that deep tissue injury initiates subdermally and that its primary cause involves more than localised tissue ischemia only. Recent investigation of Stekelenburg at al. showed that a complex interplay with prolonged excessive deformation potentially catalyses the irreversible process of tissue breakdown (Stekelenburg et al., 2008, Stekelenburg et al., 2007). Although the exact cause has not completely been revealed, it has been accepted that periodic pressure relief is needed to recover the buttock tissue from continuous deformation and impairment of tissue perfusion.

To reduce incidence of pressure ulcers, common applications are based on seating surfaces that distribute the buttock pressure over a larger area and decrease peak pressures under the ischial region. Although the overall buttock pressure will reduce significantly, it is unlikely that this alleviates the ischial buttock tissue enough to maintain tissue perfusion and fully prevent pressure ulcer onset. When individuals cannot reposition themselves and static seating surfaces do not relieve buttock pressure sufficiently, dynamic systems that adjusts the seating surface are useful to redistribute buttock load and to maintain tissue perfusion (Makhsous et al., 2007c, Stockton and Rithalia, 2007). However, it must never be the intention to apply dynamic seating surfaces and completely replace the need for postural movement. We therefore strive to redistribute buttock load from automatic chair adjustments that change body posture periodically.

Because pelvis alignment directly affects body posture (Janssen-Potten et al., 2001, Koo et al., 1996, Lucas et al., 2005, Sprigle et al., 2003) and buttock load (Maltais et al., 1999, Hobson and Tooms, 1992, Koo et al., 1996, Tam et al., 2003), systems that control pelvis angle relative to the seat support could play an important role in 
pressure ulcer prevention. In a previous study (van Geffen et al., 2008), we described a seating system that rotates the pelvis in sagittal direction and we investigated the effects on seat reaction load. It seemed effective to regulate the sacral interface pressure but we were not satisfied with the capacity to regulate the pressures under the tuberosities. We therefore extended the device with an intervention that adjusts pelvis angle in the frontal plane, which has been suggested an effective technique to alternately relieve tuberal pressure from side to side (Stockton and Rithalia, 2007). The current study applies this modified system as a passive motion technique for decoupled pelvis rotation (DPR) that periodically adjusts pelvis angle independent from trunk and seat support. We investigate the effects of DPR on the loads at the buttock-seat interface and we evaluate whether this technique is applicable to regulate buttock load in sitting.

\subsection{Methods}

This study was approved by the Committee for Medical Ethics of Roessingh Rehabilitation Centre (Enschede, the Netherlands).

\subsubsection{Subjects}

Eighteen healthy male subjects (age $22.6 \pm 2.4$ years, weight $74.9 \pm 8.0 \mathrm{~kg}$, length $1.84 \pm 0.05 \mathrm{~m}$, BMI $22.1 \pm 1.9 \mathrm{~kg} / \mathrm{m}^{2}$ ) were recruited for this study. All subjects read and signed an 'informed consent', which explained the objective and experimental protocol.

\subsubsection{Decoupled Pelvis Rotation (DPR)}

Figure 5.1 shows a concept with two axes of rotation aligned within the lumbar region and under the ischial tuberosities which enables sagittal pelvis rotation independent from the trunk and seat support. This concept is based on a parallelogram on which the backrest is fixed. The configuration of the parallelogram imposes pelvic tilt and is actuated from the back part of seat. In the frontal plane, pelvis rotation is actuated from below the seat. Changing the relative height between the left and right seat part rotates the pelvis sideways around an axis between the ischial tuberosities.

\subsubsection{Experimental setup}

Experiments were performed with a computer-aided adjustable simulator chair that is instrumented with the concept for DPR (Fig. 5.2). The backrest is mounted on a sledge to allow frictionless vertical trunk translation and to align the pelvis with the parallelogram.

For accurate 3D measurement of seat reaction forces, six uni-axial load cells (FUTEK ${ }^{\circledR}$, California, USA) are mounted in the seat frame. A pressure mapping device (Tekscan ${ }^{\circledR}$ Comformat, Boston, USA) was placed over the seat to measure pressure distribution. The device consists of 1024 (32 x 32) square $(15 \mathrm{~mm} \times 15 \mathrm{~mm})$ pressure elements which were calibrated with an upper limit of $32.5 \mathrm{kPa}(250 \mathrm{mmHg})$ and had a lower threshold of $0.7 \mathrm{kPa}(5 \mathrm{mmHg})$. 
Reflective markers were placed on the chair and selected pelvis landmarks. 3D chair configuration and pelvis orientation were obtained using an infrared camera motion capturing system (VICON ${ }^{\circledR}$, Oxford, UK). To prevent skin artefacts during pelvis movement, a pelvis mold (PM) was shaped around the left and right lateral iliac crest and clamped the Anterior Superior Iliac Spines and Posterior Superior Iliac Spines for optimal fixation (Fig. 5.3). To prevent problems with pelvis marker visibility, an inertial sensor (MT ${ }^{\circledR}$, Xsens, Enschede, the Netherlands) was attached on the PM as an alternative to estimate pelvis orientation. The line of gravity was used to derive sensor inclination that we related to the orientation of the PM as shown in fig. 5.3.

\subsubsection{Data Analyses}

The $x-z$ and $y-z$ planes of the global reference frame $(G)$ aligned respectively the frontal and sagittal planes of the simulator chair. The local pelvis frame $\left(R_{p}\right)$ was constructed from four markers placed on the PM (Fig 5.3). Two markers were placed on the left and right Anterior Superior Iliac Spines (LASIS and RASIS) and two markers (LSIS and RSIS) on the line between the Anterior- and Posterior Superior Iliac Spines. The $x$-axis of $R_{p}$ runs from the LASIS to the RASIS. The z-axis points cranially and is normal to the plane containing the LASIS, RASIS and the midpoint between LSIS and RSIS.

The y-axis is the cross product of the $\mathrm{z}$ - and $\mathrm{x}$-axis. In case of problems with pelvis marker visibility, information from the inertial sensor was used to construct a local sensor frame $\left(\mathrm{R}_{\mathrm{i}}\right)$ that we related to $\mathrm{R}_{\mathrm{p}}$ (Fig. 5.3). The local frames for the backrest $\left(\mathrm{R}_{1}\right)$, parallelogram $\left(\mathrm{R}_{2}\right)$ and seat support $\left(\mathrm{R}_{3}\right)$ were constructed from angular chair configurations as shown in figure 5.2.

The origin $[0,0,0]$ of $R_{3}$ lies between seat parts on top of the axis for sagittal pelvis adjustment. The orientation of $\mathrm{R}_{\mathrm{p}}$ relative to $\mathrm{R}_{3}$ defined pelvis angle in the sagittal and frontal plane by $\alpha_{x}$ and $\alpha_{y}$ respectively. In case of problems with pelvis marker visibility, $R_{i}$ relative to $R_{3}$ defined pelvis orientation.
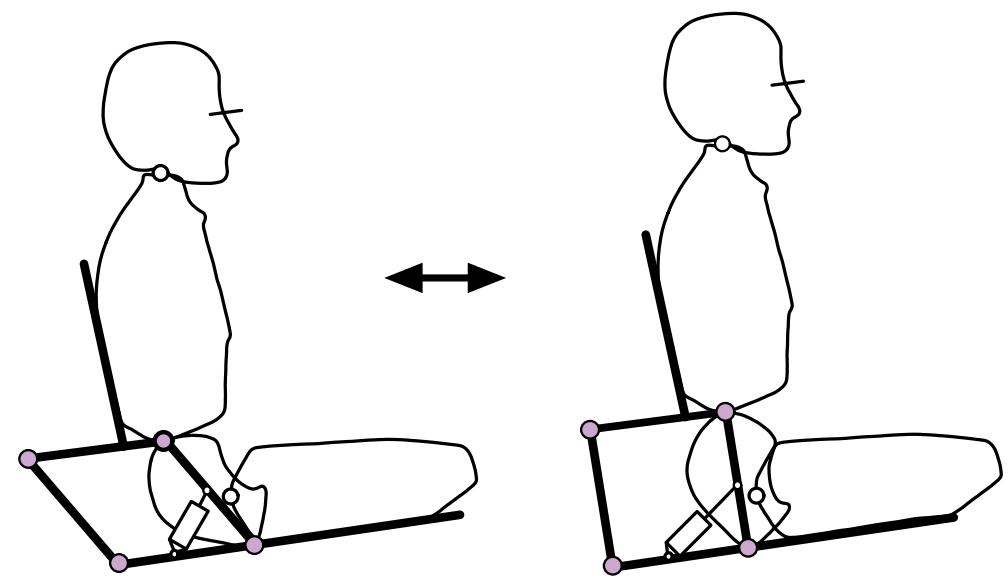

Figure 5.1: Decoupled pelvis adjustment in the sagittal plane. The backrest is fixed on a parallelogram that rotates the pelvis around a pivot under the ischial tuberosities when adjusting its configuration. 

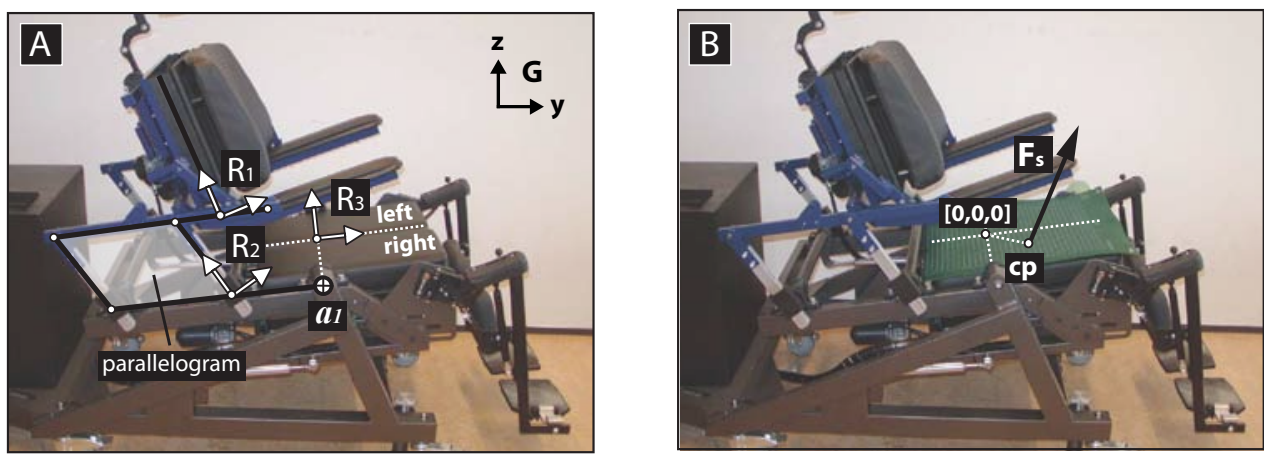

Figure 5.2: Experimental setup with a sagittal view of the computer-aided adjustable simulator chair. A: Simulator chair instrumented with the concept for decoupled pelvis adjustment. The configuration of the parallelogram and the height between the left and right seat parts are adjustable to rotate the pelvis in the sagittal and frontal plane respectively. $R_{1}, R_{2}$ and $R_{3}$ defined the local coordinate frames for the backrest, parallelogram and seat. The origin of $R_{3}$ lies between seat parts on top of the axis $\left(a_{1}\right)$ for sagittal pelvis adjustment. $G$ defines the global reference frame. B: A pressure mapping device was place over the seat to measure buttock interface pressure distribution. Six uni-axial load cells are mounted in the seat to measure seat reaction force $\left(F_{s}\right)$ and centre of pressure $(c p)$ relative to $R_{3}$ with the origin in $[0,0,0]$.

The analogue force data was used to calculate the seat reaction forces $\left(\mathrm{F}_{\mathrm{s}}\right)$ and centre of pressure (cp) relative to $\mathrm{R}_{3}$. Components of $\mathrm{F}_{\mathrm{s}}\left(\mathrm{F}_{\mathrm{sx}}, \mathrm{F}_{\mathrm{sy}}\right.$ and $\left.\mathrm{F}_{\mathrm{sz}}\right)$ and $\mathrm{cp}\left(\mathrm{cp}_{\mathrm{x}}, \mathrm{cp}_{\mathrm{y}}\right.$ and $\mathrm{cp}_{\mathrm{z}}$ ) are expressed in $\mathrm{R}_{3}$ and align the $\mathrm{x}^{-}, \mathrm{y}^{-}$, and $\mathrm{z}$-axis respectively. Buttock interface pressure distribution was derived from the analogue pressure data as shown in figure 5.4. Left and right tuberal load $\left(\mathrm{P}_{\mathrm{tr}}\right.$ and $\left.\mathrm{P}_{\mathrm{t}}\right)$ and sacral load $\left(\mathrm{P}_{\mathrm{s}}\right)$ defined the average pressures as measured within square areas $(60 \mathrm{~mm} \times 60 \mathrm{~mm})$ under the tuberosities and sacrum.

\subsubsection{Experimental protocol}

Prior to the experiment, an empty seated force calibration zero-measurement was performed. Subjects were seated with their tuberosities on the pivot for sagittal pelvis rotation. Ischial peak pressure mapping was used to monitor the tuberosities when positioning the subject in the chair and aligning the anatomical sagittal plane with the global $y$-z plane. The footrests were set perpendicular to the seat and adjusted so the thighs were supported except for the popliteal space. The initial angles for the seat, parallelogram and backrest were set to $12.5^{\circ}, 37.5^{\circ}$ and $27.5^{\circ}$ respectively.

Figure 5.5 shows the protocol for sagittal and frontal pelvis adjustments. Pelvis rotation in sagittal direction was induced from parallelogram adjustment over a range of $35^{\circ}\left(37.5 \pm 17.5^{\circ}\right)$, and in frontal direction from seat part adjustment over $60 \mathrm{~mm}(0 \pm 30 \mathrm{~mm})$.

Each trial started with a static reference measurement to predefine initial pelvis angles, the exact locations of the tuberosities and sacrum, and to relate $R_{i}$ to $R_{p}$. Subjects were asked to undergo chair movements in a passive way. This meant that they were not allowed to perform any activity that could influence body movement as induced by the chair. Each trial involved two minutes of quasi-static $(1 / 30 \mathrm{~Hz})$ chair 
adjustments. Data was only collected during the last 60 seconds which included two movement cycles. A typical measurement of pelvis angles $\left(\alpha_{x}\right.$ and $\left.\alpha_{y}\right)$, centre of pressure $\left(\mathrm{cp}_{\mathrm{x}}\right.$ and $\left.\mathrm{cp}_{\mathrm{y}}\right)$, seat reaction forces $\left(\mathrm{F}_{\mathrm{sx}}, \mathrm{F}_{\mathrm{sy}}\right.$ and $\left.\mathrm{F}_{\mathrm{sz}}\right)$ and buttock pressures $\left(\mathrm{P}_{\mathrm{tr}}\right.$, $\mathrm{P}_{\mathrm{tl}}$ and $\mathrm{P}_{\mathrm{s}}$ ) in sagittal pelvis rotation are shown in figure 5.6.
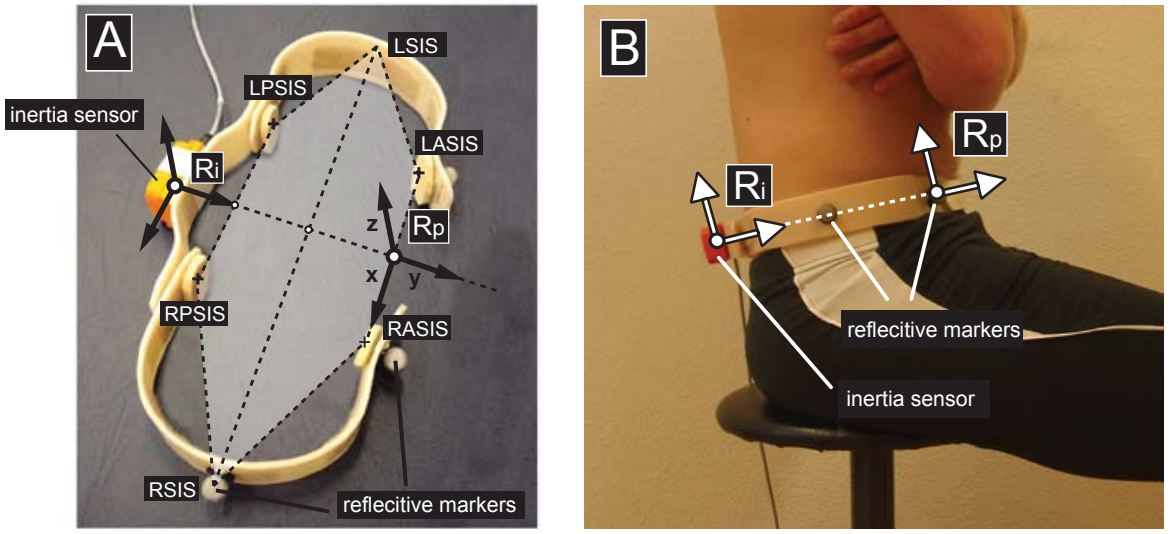

Figure 5.3: A: Pelvis mold with reflective markers and inertial sensor. The local pelvis frame $\left(\mathrm{R}_{\mathrm{p}}\right)$ is constructed from the reflective markers that were placed on the RASIS, LASIS, RSIS and LSIS. The RPSIS and LPSIS lie in the $x-y$ plane. The inertial sensor is attached in a way that the local sensor frame $\left(\mathrm{R}_{\mathrm{i}}\right)$ aligns $\mathrm{R}_{\mathrm{p}}$. B: The pelvis mold clamped the RASIS, LASIS, RPSIS and LPSIS and was shaped around the left and right lateral iliac crest. Ri was used as an alternative measure for $R_{p}$ when the reflective pelvis markers were not visible.

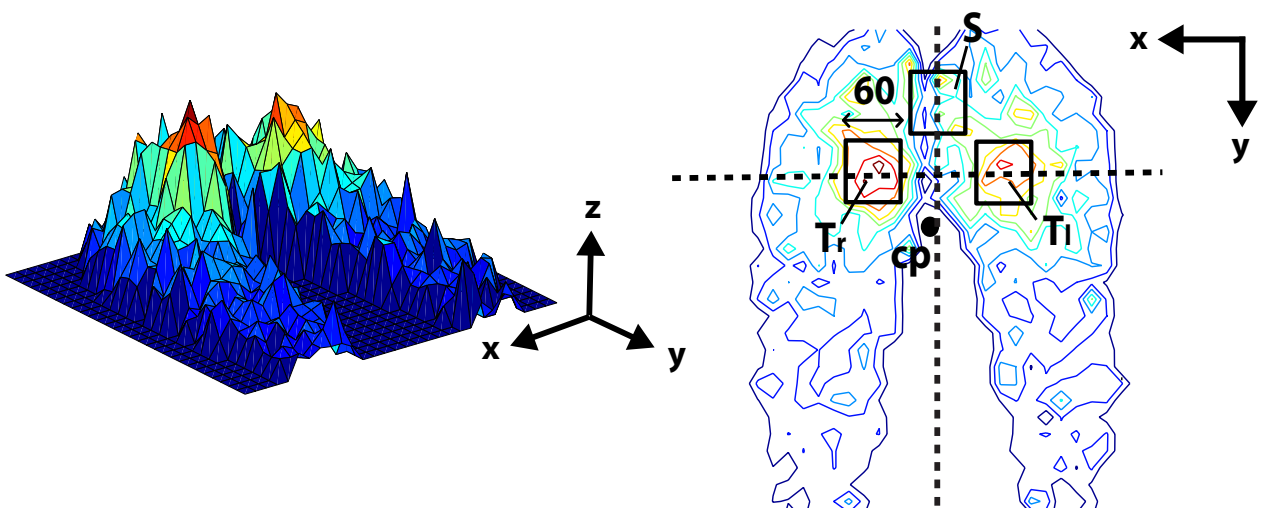

Figure 5.4: Buttock interface pressure distribution. $\mathrm{Tr}, \mathrm{Tl}$ and $\mathrm{S}$ represent the square areas $(60 \mathrm{~mm} \times 60 \mathrm{~mm})$ in which the average interface pressures were measured for the right and left ischial tuberosities and sacrum respectively. The horizontal dashed line represents the axis for sagittal pelvis adjustment en runs through $\mathrm{Tr}$ and $\mathrm{Tl}$. The vertical dashed line runs through the middle of the seat and divides the left and right seat part. The intersection between the dashed lines defines the origin from which centre of pressure (cp) was measured. 


\subsubsection{Statistics}

During pelvis movement, the initial values and ranges for all quantities $\left(\alpha_{x}, \alpha_{y}, F_{s x}\right.$, $\mathrm{F}_{\mathrm{sy}}, \mathrm{F}_{\mathrm{sz}}, \mathrm{cp}_{\mathrm{x}}, \mathrm{cp}_{\mathrm{y}}, \mathrm{P}_{\mathrm{tr}}, \mathrm{P}_{\mathrm{tl}}$ and $\mathrm{P}_{\mathrm{s}}$ ) were derived and averaged over all subjects. Pressure changes under the tuberosities $\left(\Delta \mathrm{P}_{\mathrm{tr}}\right.$ and $\left.\Delta \mathrm{P}_{\mathrm{t} t}\right)$ and sacrum $\left(\Delta \mathrm{P}_{\mathrm{s}}\right)$ were expressed relative to its initial value and grouped in a bar diagram. A multiple comparison one-way ANOVA was then performed to compare between the effects of sagittal and frontal pelvis rotation on $\Delta \mathrm{P}_{\mathrm{tr}}, \Delta \mathrm{P}_{\mathrm{tl}}$ and $\Delta \mathrm{P}_{\mathrm{s}}$. Furthermore, all quantities were normalized to its total range. Normalized quantities for seat reaction load $\left({ }^{n} F_{s x},{ }^{n} F_{s y},{ }^{n} F_{s z},{ }^{n} \mathrm{Cp}_{x},{ }^{n} \mathrm{Cp}_{y}\right.$, ${ }^{n} P_{t r},{ }^{n} P_{t l}$ and $\left.{ }^{n} P_{s}\right)$ were related to normalised pelvis angle $\left({ }^{n} \alpha_{x}\right.$ and $\left.{ }^{n} \alpha_{y}\right)$. For sagittal pelvis adjustment, normalized quantities were related to ${ }^{n} \alpha_{x}$, and for frontal pelvis adjustment to ${ }^{n} \alpha_{y}$. Linear relations were assumed and the coefficients of correlation $(r)$ with accompanying significance (p) were derived. We then calculated the coefficients of determination $\left(\mathrm{r}^{2}\right)$ which gives us an indication about the strength of the relation. Quantities with high coefficients of determination $\left(\mathrm{r}^{2}>0.8\right)$ involve a strong relation with pelvis alignment and are considered regulable from pelvis adjustment.

\subsection{Results}

Initial values (initial $\pm \mathrm{sd}$ ), ranges $(\Delta \pm \mathrm{sd})$ and coefficients of determination $\left(\mathrm{r}^{2}\right)$ are shown in table 5.1 for all quantities. $\mathrm{P}_{\mathrm{tr}}, \mathrm{P}_{\mathrm{tl}}$ and $\mathrm{P}_{\mathrm{s}}$ changed $(\Delta) 125 \pm 22 \mathrm{~Pa} / \mathrm{kg}(75.0 \pm$ $13.2 \%), 106 \pm 25 \mathrm{~Pa} / \mathrm{kg}(57.9 \pm 13.7 \%)$ and $38 \pm 14 \mathrm{~Pa} / \mathrm{kg}(30.6 \pm 11.3 \%)$ in frontal pelvis rotation, and $33 \pm 14 \mathrm{~Pa} / \mathrm{kg}(17.9 \pm 7.6 \%), 28 \pm 12 \mathrm{~Pa} / \mathrm{kg}(14.8 \pm 6.3 \%)$ and $99 \pm 29 \mathrm{~Pa} / \mathrm{kg}$ $(80.5 \pm 23.6 \%)$ in sagittal pelvis rotation. Grouped in a bar diagram, pressure changes under the tuberosities $\left(\Delta \mathrm{P}_{\mathrm{tr}}\right.$ and $\left.\Delta \mathrm{P}_{\mathrm{tl}}\right)$ and sacrum $\left(\Delta \mathrm{P}_{\mathrm{s}}\right)$ are shown figure 5.7. Pressure relief and increase for $\Delta \mathrm{P}_{\mathrm{tr}}$ and $\Delta \mathrm{P}_{\mathrm{tl}}$ are significantly $(\mathrm{p}<0.05)$ larger in frontal pelvis rotation, and for $\Delta \mathrm{P}_{\mathrm{s}}$ in sagittal pelvis rotation.

Normalised quantities for seat reaction load were related to normalised pelvis angle for all subjects and linear best-fits together with the coefficients of determination $\left(\mathrm{r}^{2}\right)$ were derived as presented in figure 5.8. A selection of the results is shown, which gives a good indication about the variability between subjects. Table 5.1 shows that in frontal pelvis rotation high values for $\mathrm{r}^{2}(>0.8)$ were found for $\mathrm{cp}_{\mathrm{x}}, \mathrm{F}_{\mathrm{sx}}, \mathrm{P}_{\mathrm{tr}}$ and $\mathrm{P}_{\mathrm{tl}}$, and in sagittal pelvis rotation high values were found for $\mathrm{cp}_{\mathrm{y}}$ and $\mathrm{P}_{\mathrm{s}}$.

\subsection{Discussion}

This study described a passive motion technique that adjusts pelvis angle independent from the trunk and seat support, and evaluates whether so-called decoupled pelvis rotation (DPR) might be applicable to regulate buttock load associated with pressure ulcer development in prolonged static sitting.

In frontal and sagittal direction, the pelvis rotated up to $9^{\circ}$ and $19^{\circ}$ respectively. Strong relations $\left(r^{2}>0.8\right)$ were found between pelvis rotation and all quantities of buttock load, except for the normal force $\left(\mathrm{F}_{\mathrm{sz}}\right)$ and sagittal shear $\left(\mathrm{F}_{\mathrm{sy}}\right)$. To influence buttock interface pressures, we found that sagittal pelvis adjustment is most effective to regulate sacral pressure and that frontal pelvis adjustment is most effective to regulate tuberal pressure. Sacral and tuberal pressure reliefs were measured up to 

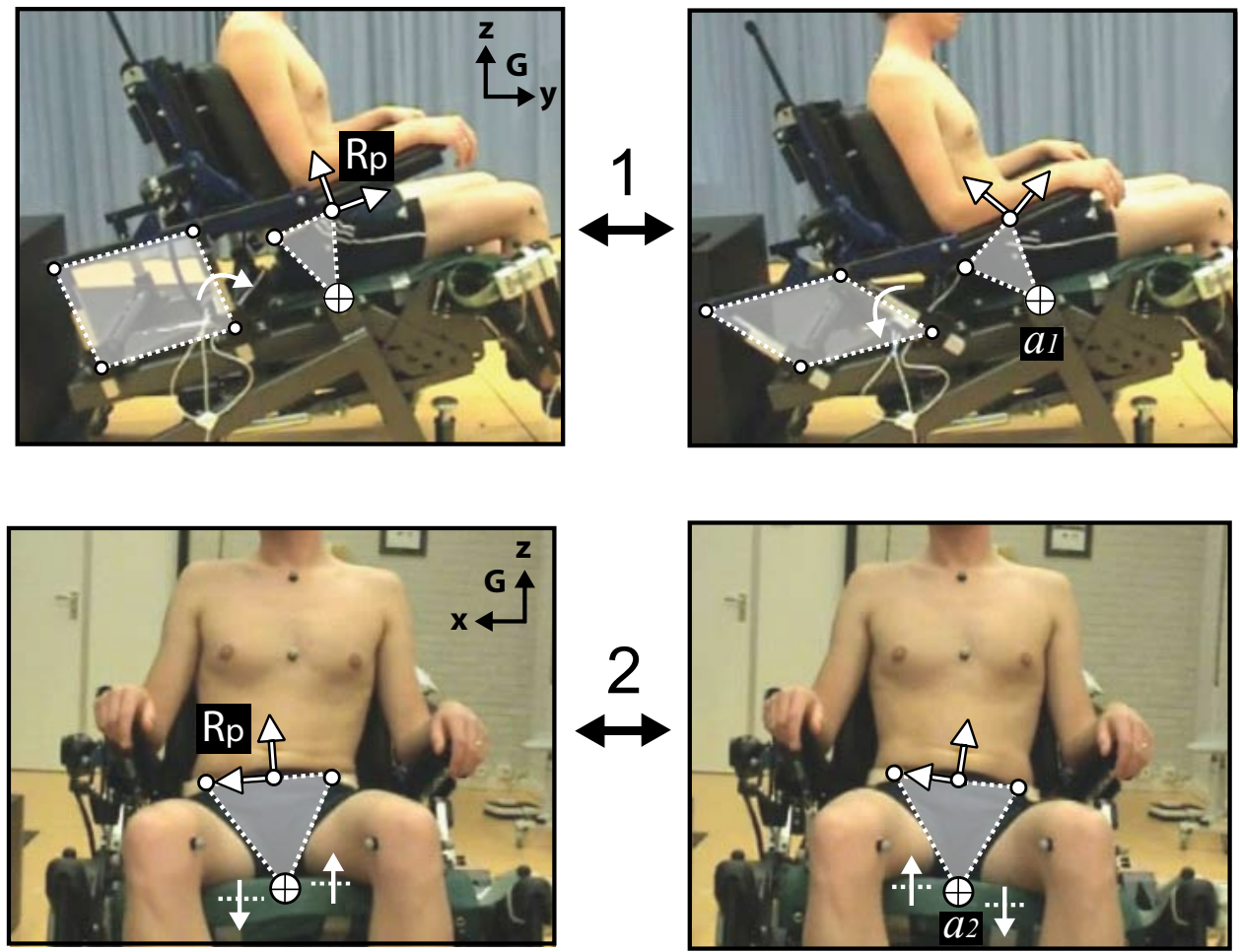

Figure 5.5: Protocol for sagittal pelvis adjustment (1) and frontal pelvis adjustment (2). Pelvis angle is derived from the orientation of the local pelvis frame $\left(R_{p}\right)$. G defines the global reference frame. Adjusting the configuration of the parallelogram rotates the pelvis in the sagittal plane around an axis under the ischial tuberosities (a1). Adjusting the height between the left and right seat parts rotates the pelvis in the frontal plane around an axis between the ischial tuberosities (a2).

$40 \%$ and $34 \%$ respectively.

Strong linear effects were also found on the displacement of centre of pressure (cp) in both directions. Right seat part elevation pushes the right buttock upward and tilts the pelvis to the left. The load under the right tuberal area increased and cp travelled to the right. Lateral cp shifts without centre of mass $(\mathrm{cm})$ displacement explained the increase in lateral shear $\left(\mathrm{F}_{\mathrm{sx}}\right)$. In the sagittal plane, cp shifts were accompanied with $\mathrm{cm}$ displacements and caused the resultant reaction force $\left(\mathrm{F}_{\mathrm{s}}\right)$ to shift parallel rather that to change direction. This also explains the minimal effect on $\mathrm{F}_{\mathrm{sy}}$. That no significant effect was found on $\mathrm{F}_{\mathrm{sy}}$ was supported by a previous study (van Geffen et al., 2008) where we suggested that $\mathrm{F}_{\mathrm{sy}}$ had to be regulated by inclining the seat support rather than adjusting the pelvis in sagittal direction. This previous study also showed that sagittal pelvis adjustments were not effective to regulate the interface pressures under the tuberosities. In the present study, minimal effects on $\mathrm{P}_{\mathrm{tr}}$ and $\mathrm{P}_{\mathrm{tl}}$ were also found. The contact pressures under the right and left tuberosities changed only $17.9 \%$ and $14.8 \%$ from the initial values. This relative small effect was ascribed to the dimensions $(60 \mathrm{~mm} \times 60 \mathrm{~mm})$ of the tuberal areas. The observed $\mathrm{cp}$ 
sagittal pelvis rotation
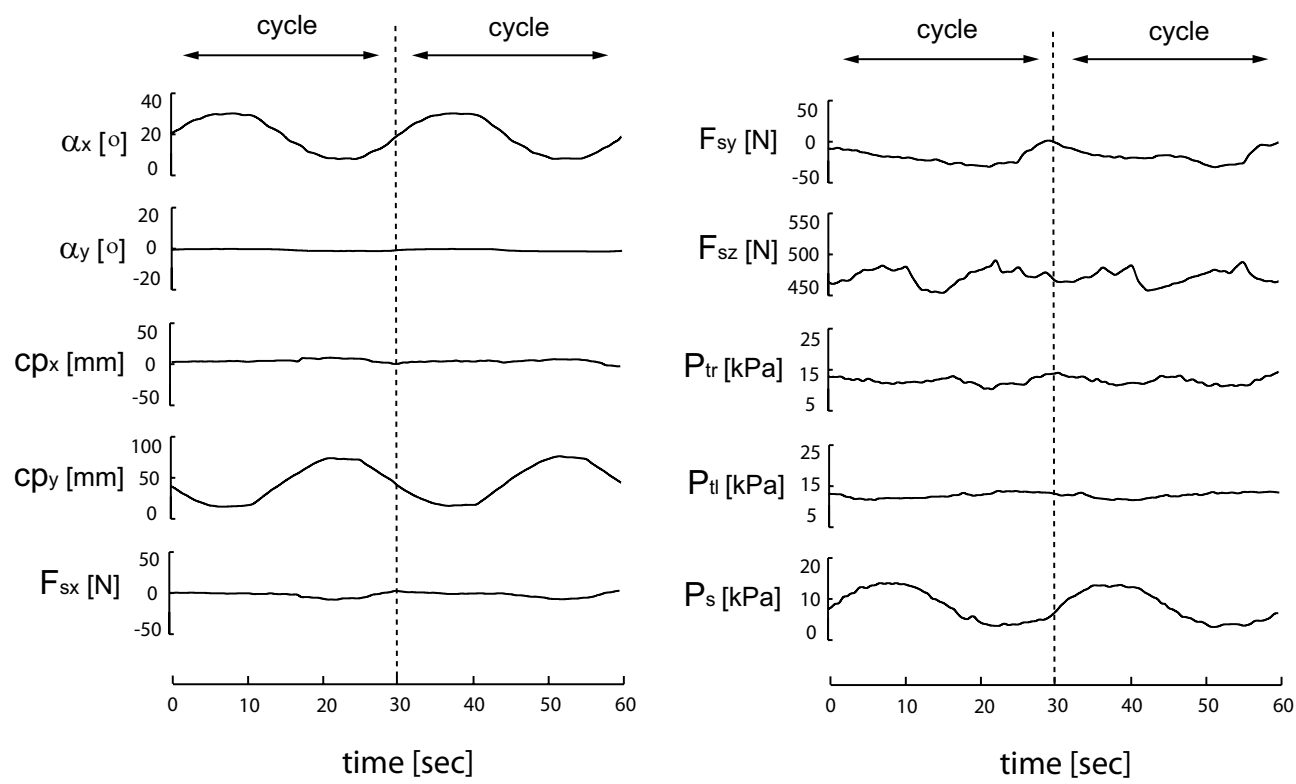

Figure 5.6: A typical measurement of pelvis angles $\left(\alpha_{\mathrm{x}}\right.$ and $\left.\alpha_{\mathrm{y}}\right)$, centre of pressure $\left(\mathrm{cp}_{\mathrm{x}}\right.$ and $\left.\mathrm{cp}_{\mathrm{y}}\right)$, seat reaction forces $\left(\mathrm{F}_{\mathrm{sx}}, \mathrm{F}_{\mathrm{sy}}\right.$ and $\left.\mathrm{F}_{\mathrm{sz}}\right)$ and buttock pressures $\left(\mathrm{P}_{\mathrm{tr}}, \mathrm{P}_{\mathrm{tl}}\right.$ and $\left.\mathrm{P}_{\mathrm{s}}\right)$ during sagittal pelvis rotation.

displacement of $54 \mathrm{~mm}$ roughly implies that the ischial tuberosities rolled within the defined areas causing that only minimal pressure changes were measured. Smaller areas will increase the amount of relative pressure change but also requires a more accurate localization of vulnerable places for tissue breakdown.

Apart from automatic chair modification, leaning forward and pushing up from the armrests are effective movement strategies to relieve buttock load periodically and are therefore part of many repositioning programs that prescribe wheelchair-users to undertake body movement every 15 minutes (Agency for Health Care Policy and Research (AHCPR), 1994). Ischial peak pressures relieve up to $40 \%$ when leaning forward $45^{\circ}$ and even up to $80 \%$ when leaning forward completely (Henderson et al., 1994, Koo et al., 1996). For full recovery of localised tissue oxygenation and perfusion, there is evidence that for some individuals the buttock must be unloaded for at least two minutes (Coggrave and Rose, 2003, Makhsous et al., 2007b, Bader, 1990). This requires good upper-body strength and is therefore not feasible for many impaired wheelchair-users. When individuals cannot reposition body posture functionally, automatic chair recline (tilt-in-space) is an effective alternative for periodic pressure relief. It is reported that the ischial peak pressures relieve 32\%, 40\% and $47 \%$ when reclining the chair $35^{\circ}, 50^{\circ}$ and $65^{\circ}$ respectively (Henderson et al., 1994, Sprigle et al., 2007). Although the benefits of body movement on pressure relief have been demonstrated, many wheelchair-users do not concord with prescribed movement programs or other clinical repositioning advice, even when they posses adequate cognitive and physical ability to relieve buttock pressure from either functional 
Table 5.1: Initial values (initial $\pm s d)$, ranges $(\Delta \pm \mathrm{sd})$ and coefficients of determination $\left(\mathrm{r}^{2}\right)$ for all quantities $\left(\alpha_{\mathrm{x}}, \alpha_{\mathrm{y}}, \mathrm{F}_{\mathrm{sx}}, \mathrm{F}_{\mathrm{sy}}, \mathrm{F}_{\mathrm{sz}}, \mathrm{cp}_{\mathrm{x}}, \mathrm{cp}_{\mathrm{y}}, \mathrm{P}_{\mathrm{tr}}, \mathrm{P}_{\mathrm{tl}}\right.$ and $\left.\mathrm{P}_{\mathrm{s}}\right)$ in sagittal and frontal pelvis rotation.

\begin{tabular}{|c|c|c|c|c|c|c|c|}
\hline & & frontal pel & rotation & & sagittal pel & rotation & \\
\hline & & Initial $\pm \mathrm{sd}$ & $\Delta \pm \mathrm{sd}$ & $\mathrm{r}^{2}$ & Initial \pm sd & $\Delta \pm \mathrm{sd}$ & $\mathrm{r}^{2}$ \\
\hline$\alpha_{x}$ & [deg] & $23 \pm 5$ & $2 \pm 1$ & - & $22 \pm 5$ & $19 \pm 2$ & 1.00 \\
\hline$\alpha_{y}$ & [deg] & $-1 \pm 2$ & $9 \pm 1$ & 1.00 & $-1 \pm 2$ & $1 \pm 1$ & - \\
\hline $\mathrm{cp}_{\mathrm{x}}$ & {$[\mathrm{mm}]$} & $8 \pm 10$ & $63 \pm 11$ & 0.96 & $7 \pm 7$ & $4 \pm 2$ & 0.00 \\
\hline $\mathrm{cp}_{\mathrm{y}}$ & {$[\mathrm{mm}]$} & $57 \pm 20$ & $10 \pm 4$ & 0.14 & $54 \pm 19$ & $54 \pm 7$ & 0.98 \\
\hline $\mathrm{F}_{\mathrm{sx}}$ & {$[\mathrm{N} / \mathrm{kg}]$} & $-0.02 \pm 0.09$ & $0.80 \pm 0.10$ & 0.96 & $0.00 \pm 0.10$ & $0.08 \pm 0.04$ & 0.17 \\
\hline $\mathrm{F}_{\mathrm{sy}}$ & {$[\mathrm{N} / \mathrm{kg}]$} & $-0.15 \pm 0.33$ & $0.09 \pm 0.03$ & 0.00 & $-0.01 \pm 0.36$ & $0.70 \pm 0.30$ & 0.36 \\
\hline $\mathrm{F}_{\mathrm{sz}}$ & {$[\mathrm{N} / \mathrm{kg}]$} & $6.83 \pm 0.47$ & $0.25 \pm 0.09$ & 0.01 & $7.12 \pm 0.53$ & $0.65 \pm 0.14$ & 0.00 \\
\hline $\mathrm{P}_{\mathrm{tr}}$ & {$[\mathrm{Pa} / \mathrm{kg}]$} & $167 \pm 28$ & $125 \pm 22$ & 0.95 & $184 \pm 26$ & $33 \pm 14$ & 0.02 \\
\hline $\mathrm{P}_{\mathrm{tl}}$ & {$[\mathrm{Pa} / \mathrm{kg}]$} & $183 \pm 23$ & $106 \pm 25$ & 0.94 & $189 \pm 23$ & $28 \pm 12$ & 0.01 \\
\hline $\mathrm{P}_{\mathrm{s}}$ & {$[\mathrm{Pa} / \mathrm{kg}]$} & $124 \pm 24$ & $38 \pm 14$ & 0.07 & $123 \pm 24$ & $99 \pm 29$ & 0.93 \\
\hline
\end{tabular}

Bold values for $\mathrm{r}^{2}$ involve strong correlations $\left(\mathrm{r}^{2}>0.8\right)$ with pelvis rotation

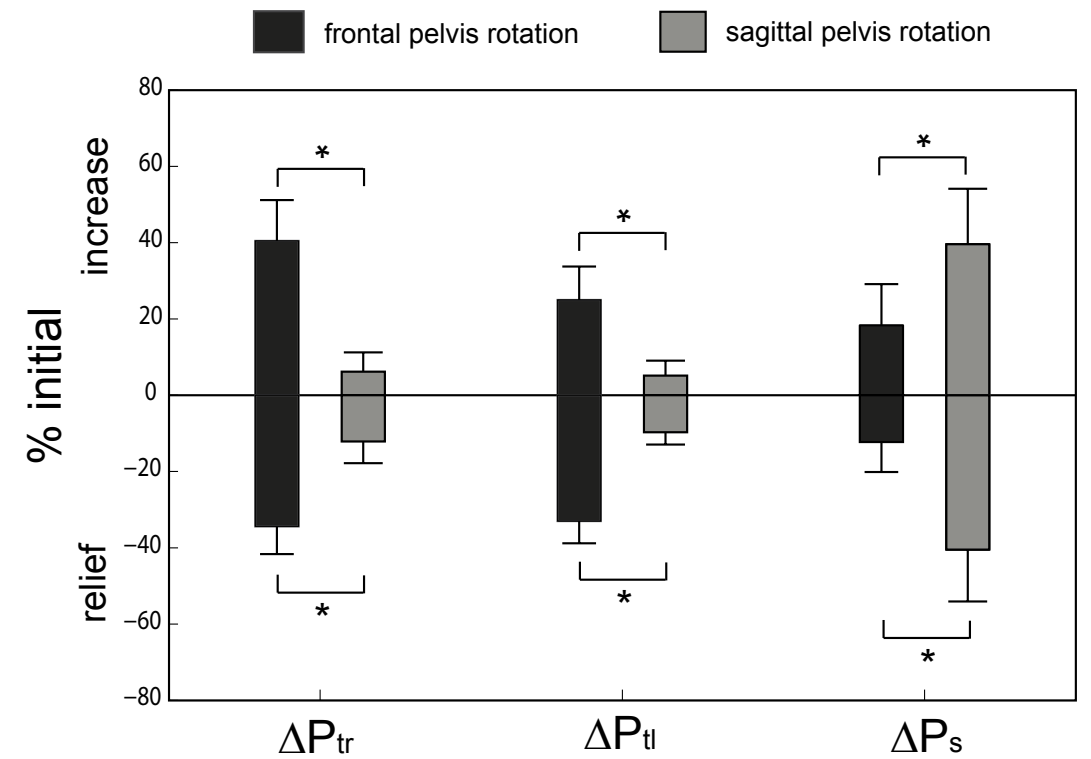

Figure 5.7: Bar diagrams of pressure changes under the tuberosities $\left(\Delta \mathrm{P}_{\mathrm{tr}}\right.$ and $\left.\Delta \mathrm{P}_{\mathrm{tI}}\right)$ and sacrum $\left(\Delta \mathrm{P}_{\mathrm{s}}\right)$ expressed relative to its initial value (\%initial) and divided into the amount of pressure relief and increase during frontal and sagittal pelvis rotation. *Statistically significant $(\mathrm{p}<0.05)$ difference between groups (frontal and sagittal pelvis rotation). 


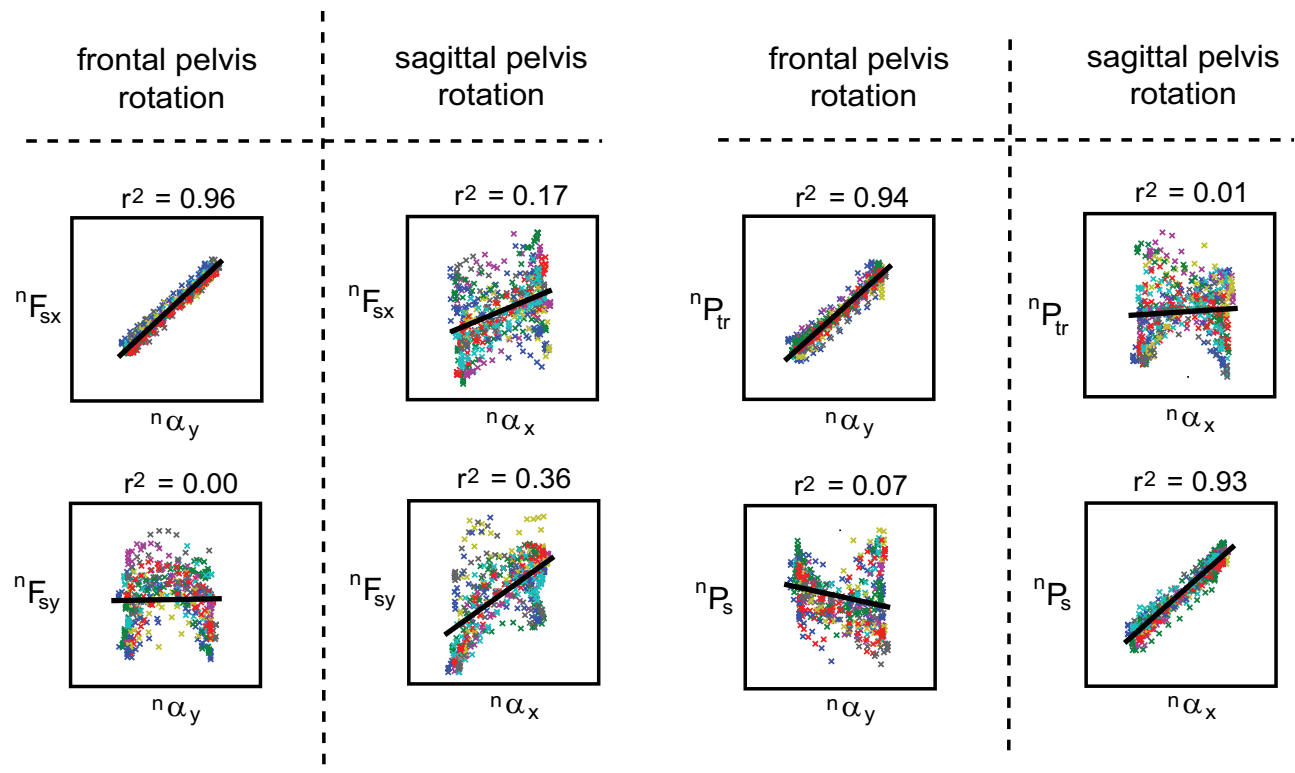

Figure 5.8: A selection of the results in which some normalised quantities for seat reaction load $\left({ }^{n} F_{s x},{ }^{n} F_{s y},{ }^{n} P_{t r}\right.$ and $\left.{ }^{n} P_{s}\right)$ are related to normalised pelvis angle $\left({ }^{n} \alpha_{x}\right.$ and $\left.{ }^{n} \alpha_{y}\right)$ for all subjects. Linear best-fits together with the coefficients of determination $\left(\mathrm{r}^{2}\right)$ are shown, which give us a good indication about the variability between subjects.

repositioning or automatic chair modification (Stockton and Parker, 2002). Because we found that DPR could unload the ischial buttock region up to $40 \%$, it might be an applicable intervention to satisfy prescribed clinical recommendation regarding the benefits of periodic pressure relief in daily wheelchair use.

The present study uses mean pressure measurement on predefined areas under the sacrum and tuberosities to assess the manipulation of ischial buttock tissue during chair modification. In relation to other measures (e.g. mean pressures, peak pressures and peak pressure gradient) for ischial pressure distribution (Maltais et al., 1999), we believe that mean pressures in predefined ischial peak pressure areas give a good representation of ischial pressure distribution without losing relevant information. However, it has been recognized that the external load provides only little information about the internal mechanical and physiological tissue conditions (Oomens et al., 2003). Finite element studies have shown that there is no distinct relation between the interface pressure and internal buttock load (Oomens et al., 2003, Linder-Ganz et al., 2007b, Linder-Ganz et al., 2005, Linder-Ganz et al., 2008). Continuous information about the internal deformation of ischial buttock tissue might therefore be useful to protect wheelchair-users from pressure ulcers onset. However, technical limitations make that non-invasive direct measurement of the internal tissue conditions is currently not feasible. Linder-Ganz at al. developed a patient-specific real-time finite element model and reported that this new approach might be applicable to monitor stress/strain concentrations in deep muscle layers (Linder-Ganz et al., 2005). Real-time finite element analysis in combination with simultaneous measurement of seat reaction forces might therefore benefit the described technique for DPR to regulate the internal buttock tissue conditions and to 
prevent deep tissue injury from prolonged static wheelchair sitting.

It is hard to predict to what extent the buttock tissue can sustain certain deformation before tissue degeneration takes place and how often the pelvis must be adjusted to improve ischial buttock tissue viability. A number of researchers have taken much trouble to identify reliable thresholds for minimal pressure and duration which consistently generates tissue necrosis (Bouten et al., 2003, Linder-Ganz et al., 2006, Stekelenburg et al., 2007, Gefen et al., 2008b). However, no reliable and clinically applicable indicator has yet been found that predicts pressure ulcers onset in deep muscle tissue. Because able-bodied individuals do not suffer pressure ulcers due to continuous body movement, healthy sitting behaviour might provide interesting information about the extent and frequency of postural change which potentially prevents physical discomfort such as pressure ulcers. US Department of Health (Agency for Health Care Policy and Research (AHCPR), 1994) recommends wheelchairusers to relieve buttock pressure every hour and to shift body weight every 15 minutes. In a recent study, Linder-Ganz et al. evaluated healthy sitting behaviour and reported significant body movement when quantifying postural change in prolonged wheelchair sitting (Linder-Ganz et al., 2007a). The frequency of postural change for healthy individuals seemed higher than recommended by the US Department of Health. Based on pressure changes under the ischial tuberosities, Linder-Ganz et al. found that healthy individuals moved approximately every 6 minutes in the sagittal plane and every 9 minutes in the frontal plane. For continuous regulation of buttock load in sitting, it might therefore be interesting to evaluate the consequences for ischial buttock tissue viability when to apply DPR with various frequencies and extent.

\subsection{Conclusions}

We conclude that DPR might be an applicable passive motion technique that regulates buttock load associated with pressure ulcer development in prolonged static wheelchair sitting. For clinical use, this technique is still to be evaluated on individuals who cannot functionally reposition themselves. Impairments to the neuromuscular function influence postural response (e.g. muscle spasms) from chair adjustment and make clinical investigation necessary. Experimental evaluation of the viability and mechanical conditions of the internal buttock tissue in impaired wheelchair-users also needs further attention, especially since it has been reported that the internal buttock load is different between impaired individuals and able-bodied individuals (Linder-Ganz et al., 2008). This could provide a better understanding about the role of DPR in regulation of buttock load associated with pressure ulcer onset in prolonged static sitting.

\subsection{Acknowledgements}

This study was partly funded by the Dutch Ministry of Economical Affairs, SenterNovem. The authors would like to thank the engineering company Demcon (Oldenzaal, the Netherlands) for developing the experimental simulator chair. 


\section{Chapter 6}

\section{Dynamic Tuberal Support to Regulate Ischial Buttock Load}

Resubmitted as: P. van Geffen, J. Reenalda, P.H. Veltink and H.F.J.M. Koopman, The effects of a dynamic tuberal support on ischial buttock load and pattern of blood supply (under revision) 


\begin{abstract}
Sitting acquired pressure ulcers (PU) are places of tissue breakdown that mainly occur under the ischial tuberosities (ITs). Successive durations of pressure relief help the buttock tissue recover from sustained deformation and blood-flow stagnation. A computer-aided simulator chair was developed with two adjustable tuberal support elements (TSE) integrated in a force-sensing seating plane (FSP). This study investigated the redistribution of external buttock load in relation to the pattern (i.e. dynamics) of subtuberal blood supply in sitting with a dynamic tuberal support of $1 / 60 \mathrm{~Hz}(80 \mathrm{~mm} /$ min).

Fifteen healthy male subjects were seated with their ITs on the TSE. The experiment involved periodic TSE adjustment in which buttock interface pressure was measured with the FSP and an external pressure mapping device (PMD). Light-guide tissue spectrophotometry was used for simultaneous non-invasive measurement of oxygenation and perfusion in the skin $(<2 \mathrm{~mm})$ and subcutaneous $(<8 \mathrm{~mm})$ tissue under the ITs.

TSE adjustment seemed effective to regulate centre of buttock pressure and the forces under the ITs. Differences in measurement with the FSP and PMD have been found due to Hammocking at the seat interface and inaccurate peak pressure readings. Subtuberal blood supply was inversely related to the contact load under the ITs. A rapid inflow of blood in the initial stage of tuberal unloading, followed by a gradual outflow in the rest of the movement cycle indicates that the average blood supply increases when the adjustment frequency increases. Future studies must address the influence of a dynamic tuberal support on the ischial buttock load and pattern of blood supply in impaired individuals
\end{abstract}




\subsection{Introduction}

Wheelchair-users who cannot reposition themselves often suffer from pressure ulcers (PU), which are localized places of tissue breakdown that mainly occur in the buttock tissue under the ischial tuberosities (ITs) (Bouten et al., 2003, Black et al., 2007). Current beliefs discriminate between superficial and deep PU (Quintavalle et al., 2006, Bouten et al., 2003). Superficial PU occur in the skin from sustained shear and pressure on the buttock interface, and deep PU occur from sustained internal buttock loading and are often located in the muscle tissue directly under bony structures. This latter type of injury is extremely hard to treat because it progresses outwards to the skin and when it manifests at the buttock surface it already caused severe damage to the sub-dermal tissue.

There is evidence that deep tissue breakdown initiates sub-dermally, even when comparatively low pressures exert at the buttock surface (Reswick and Rogers, 1976). Experimental research with muscle-cell cultures (Gawlitta et al., 2007, Gefen et al., 2008a, Gawlitta et al., 2008, Gefen et al., 2008b), finite element models (LinderGanz et al., 2007a, Brosh and Arcan, 2000, Ragan et al., 2002, Todd and Thacker, 1994, Lim et al., 2007, Makhsous et al., 2007a), animals (Linder-Ganz et al., 2006, Stekelenburg et al., 2006) and humans (Ohura et al., 2007, Quintavalle et al., 2006) have been preformed to reveal the exact cause, onset, location and extent of deep tissue damage from sustained internal loading. Recent studies of Stekelenburg et al. demonstrated that the onset of deep PU involves a complex interplay of prolonged ischemia together with excessive friction and shear loading due to high internal pressure gradients (Stekelenburg et al., 2008, Stekelenburg et al., 2007). Although the exact cause remains unclear, it has been recognised that successive durations of pressure relief help buttock tissue recover from sustained deformation and blood flow stagnation.

Common applications to prevent PU are based on seating surfaces that distribute the buttock pressure over a larger area and locally reduce the applied pressure under the ITs (Whittemore, 1998). The process of reshaping the seating surface for optimal pressure distribution often involves a protracted and iteratively procedure. Some computer-aided seating systems (Brienza et al., 1996a, Kwiatkowski and Inigo, 1993, Brienza et al., 1993), equipped with a matrix of adjustable force sensing support elements, have therefore been developed that automatically adjust the surface shape based on pre-programmed criteria for interface pressure and soft tissue characteristics. Although the effectiveness of designing individualized contour shaped seat surfaces have been demonstrated (Brienza et al., 1996b), systems like these are often too complex and expensive to be integrated as an assistive technology in daily wheelchair-use.

When static seating surfaces do not relieve buttock pressure enough, dynamic buttock supports appeared useful for proper pressure management (Makhsous et al., 2007b, Stockton and Rithalia, 2007, Bader, 1990). We therefore developed a computer-aided simulator chair with two adjustable support elements under the ITs (van Geffen and Reenalda, 2008). Built-in measurement of seat reaction forces allows quantitative assessment of the external buttock load during support surface manipulations. However, it has been recognized that the external buttock pressure provides only little information about the internal mechanical and physiological tissue conditions 
(Oomens et al., 2003). Finite element studies showed that there is no distinct relation between the interface pressure and internal buttock load (Oomens et al., 2003, LinderGanz et al., 2007a, Linder-Ganz et al., 2007b, Linder-Ganz et al., 2005). It has been found that comparatively low pressures at the buttock interface could still lead to high internal stress levels under the bony prominences (Reswick and Rogers, 1976). A potential threat for deep PU remains, even when the buttock interface pressure is evenly distributed.

Levels of oxygenation and perfusion are important indicators for the viability of tissue and can be used for quantitative assessment of peripheral perfusion and microcirculation in skin and subcutaneous tissue (Mathieu and Mani, 2007). Additional measurement of the ischial blood supply has therefore been suggested as being useful when quantifying the risk for PU in vivo (Black et al., 2007).

In a previous study (Reenalda et al., 2009), we investigated the effects of different alternating tuberal support strategies on skin and subcutaneous subtuberal tissue oxygenation and perfusion. Nine different support conditions of five minutes were successively imposed; four periods of static loading and unloading, followed by five periods of dynamic loading and unloading with various adjustment frequencies. We found that in sitting with static loaded tuberal support, the average blood supply was significantly lower compared to sitting with static unloaded tuberal support and dynamic tuberal support. Furthermore, a trend $(\mathrm{p}>0.05)$ was observed that the average level of skin oxygenation and subcutaneous tissue perfusion increased when the frequency of adjustment increased. This could indicate that the metabolic process of blood outflow due to tissue loading was slower than the mechanical process of blood inflow due to tissue unloading. However, we only compared the average blood supply between support conditions, and the dynamics of blood supply has therefore not yet been analysed.

The present study is complementary to this previous work. It investigates the redistribution of buttock load in combination with non-invasive measurement of subtuberal buttock tissue oxygenation and perfusion in sitting with a dynamic tuberal support of $1 / 60 \mathrm{~Hz}(80 \mathrm{~mm} / \mathrm{min})$, for which the pattern (i.e. dynamics) of blood supply in relation to the external buttock load is analysed with a more in-depth focus.

\subsection{Methods}

\subsubsection{Subjects}

The same fifteen healthy male subjects (age $27.7 \pm 3.3$ years, weight $80.5 \pm 7.4 \mathrm{~kg}$, length $1.86 \pm 0.05 \mathrm{~m}$, BMI $23.1 \pm 1.6 \mathrm{~kg} / \mathrm{m}^{2}$ ) of our previous study (Reenalda et al., 2009) participated in the present experiment. Prior to the experiment, all subjects read and signed an 'informed consent' in which the objective and experimental protocol was explained. This study was approved by the Local Committee for Medical Ethics of 't Roessingh Centre for Rehabilitation (Enschede, the Netherlands).

\subsubsection{Instrumentation}

Experiments were performed with a computer-aided simulator chair (Fig. 6.1A). Spaced according to an average inter-tuberal distance of $120 \mathrm{~mm}$ (Linder-Ganz et al., 
2007a), two force sensing support elements (Ø 90mm) were integrated in the seating surface (Fig. 1B). Both support elements were adjustable in height within a range of $40 \mathrm{~mm}$ below the seating surface $(-20 \pm 20 \mathrm{~mm})$. For accurate 3D measurement of seat reaction load, 6 uni-axial load cells (FUTEK ${ }^{\circledR}$, California, USA) were mounted under the seat support. The support cushion was upholstered with $100 \mathrm{~mm}$ of industrial foam; $60 \mathrm{~mm}$ polyether SG40 on top of $40 \mathrm{~mm}$ polypress. Round cut-outs were made in the cushion above the support elements, which were then upholstered with $50 \mathrm{~mm}$ polyether SG40 on top of 50mm polypress.

An external pressure mapping device (Teksan ${ }^{\circledR}$, Boston Massachusetts, USA) was placed over the seat surface. The device consisted of 1024 (32 x 32) square elements $(15 \times 15 \mathrm{~mm})$ and was calibrated with an upper limit of $33 \mathrm{kPa}$. The pressure elements were connected flexibly to minimize hammocking (Casey et al., 2001). The force sensing seating plane (FSP) with integrated adjustable tuberal support elements (TSE) and external pressure mapping device (PMD) is shown in figure 6.1B. Skin and subcutaneous subtuberal buttock tissue oxygenation and perfusion were simultaneously measured with two non-invasive fibre-optic probes (Fig. 1C) that we attached on the skin under the ITs. Both probes were connected with the O2C (Oxygen-to-see, LEA ${ }^{\circledR}$, Giessen, Germany) which is a diagnostic device that uses light-guide tissue spectrophotometry, a combination of laser Doppler flowmetry and diffuse reflectance spectroscopy, for simultaneous non-invasive measurement of oxygen supply in blood perfused tissue. Each probe incorporates Laser-Doppler and broadband light spectroscopy and was shaped with relative flat dimensions (Fig. 6.1C). The probe under the left IT measured skin $(<2 \mathrm{~mm})$ oxygenation and perfusion, and the probe under the right IT measured subcutaneous $(<8 \mathrm{~mm})$ oxygenation and perfusion. Oxygenation levels in the skin and subcutaneous tissue were expressed as $\mathrm{SO}_{\text {sk }}$ and $\mathrm{SO} 2_{\mathrm{sc}}$ respectively and reflect oxygen saturation of hemoglobin as a relative measure [\%]. Perfusion levels in the skin and subcutaneous tissue were expressed as $\mathrm{BF}_{\mathrm{sk}}$ and $\mathrm{BF}_{\mathrm{sc}}$ respectively and reflect the blood flow in the microcirculation as an arbitrary unit [au].

\subsubsection{Data Analysis}

The $\mathrm{x}-\mathrm{z}$ and $\mathrm{y}-\mathrm{z}$ planes of the global reference frame $(\mathrm{G})$ aligned respectively to the frontal and sagittal planes of the simulator chair. A seat coordinate frame $\left(\mathrm{R}_{\mathrm{s}}\right)$ was constructed with the $\mathrm{x}$-axis pointing to right, the $\mathrm{y}$-axis pointing anteriorly parallel to the seat, and the z-axis pointing cranially perpendicular to the seat (Fig. 6.1A, B). The origin of $\mathrm{R}_{\mathrm{s}}$ lies between the tuberal support areas. As a relative measure for tuberal load, a dispersion index (DI) was calculated that expresses the load on the TSE relative to the total load on the seat. $\mathrm{DI}_{\mathrm{f}}$ was derived from the FSP according to equation 1 in which the normal force on the TSE $\left(\mathrm{F}_{t}\right)$ was divided by the normal force on the seat $\left(\mathrm{F}_{\mathrm{n}}\right)$. DI $\mathrm{p}$ was derived from the PMD according to equation 2 in which the sum of all pressure elements on the TSE was divided by the sum of all pressure elements on the seat support.

$$
\begin{aligned}
& \boldsymbol{D}_{f}=F_{t} / F_{n} \\
& \boldsymbol{D}_{p}=\sum\left(P_{t}\right)_{i} / \sum\left(P_{s}\right)_{i}
\end{aligned}
$$




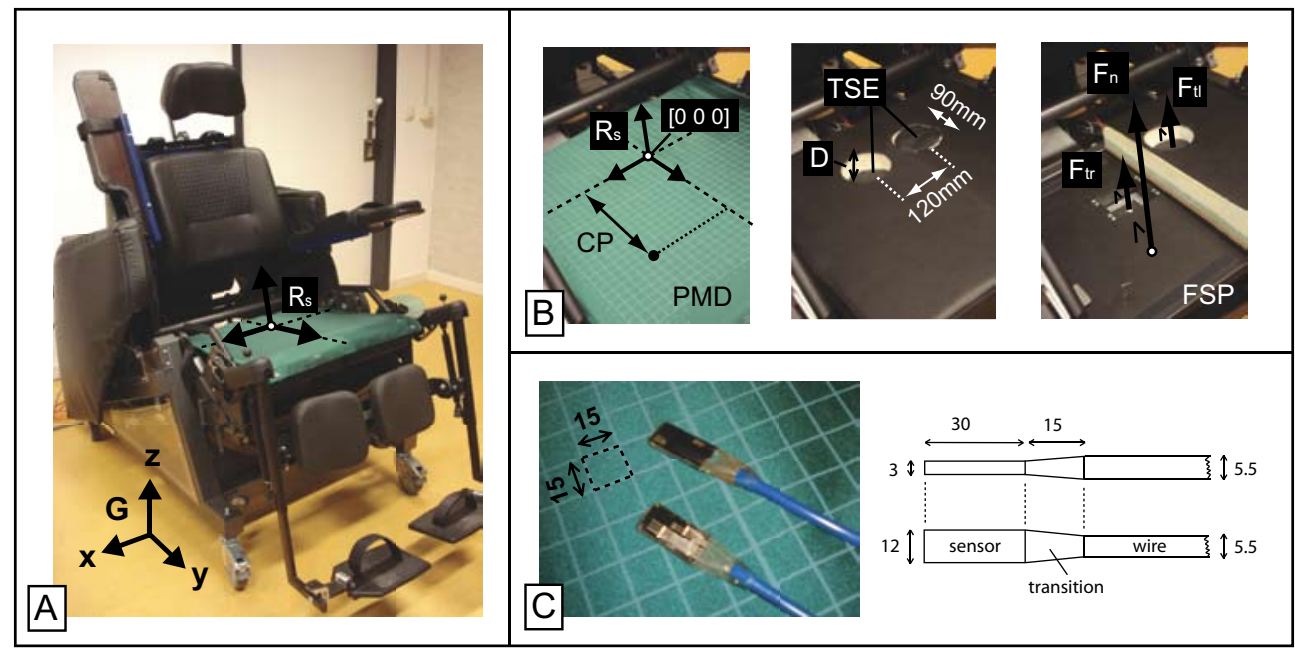

Figure 6.1: Experimental setup. A: computer-aided adjustable simulator chair with the external pressure mapping device (PMD) placed on the seat. The origin of the seat coordinate frame $\left(\mathrm{R}_{\mathrm{s}}\right)$ is located between the tuberal support elements (TSE). G defines the global reference frame. B: force sensing seating plane (FSP) with two round (Ø 90mm) adjustable force sensing tuberal support elements (TSE) spaced according to an inter-tuberal distance of $120 \mathrm{~mm}$. Maximal tuberal adjustment $(\mathrm{D}=-40 \mathrm{~mm})$ is shown. Centre of pressure $(\mathrm{CP})$ is expressed relative to the origin $\left[\begin{array}{lll}0 & 0 & 0\end{array}\right]$ and along the $y$-axis of $\mathrm{R}_{\mathrm{s}}$. The normal force on the seat $\left(\mathrm{F}_{\mathrm{n}}\right)$ and on the left and right TSE $\left(\mathrm{F}_{\mathrm{tl}}\right.$ and $\left.\mathrm{F}_{\mathrm{tr}}\right)$ are derived from the FSP. $\mathrm{F}_{\mathrm{t}}=\mathrm{F}_{\mathrm{tr}}+\mathrm{F}_{\mathrm{tl}}$. C: Shape and dimensions of the noninvasive fibre-optic probes that we attached on the skin under the left and right ITs to measure subtuberal buttock tissue oxygenation and perfusion.

Centre of pressure $(\mathrm{CP})$ was expressed along the $\mathrm{y}$-axis of $\mathrm{R}_{\mathrm{s}} \cdot \mathrm{CP}_{\mathrm{f}}$ was derived from the FSP and $\mathrm{CP}_{\mathrm{p}}$ from the PMD. Buttock interface pressures distribution in seating conditions with fully loaded and unloaded ITs are shown in figure 6.2.

\subsubsection{Experimental protocol}

Prior to the experiment, an empty seated force calibration zero-measurement was performed. Subjects were seated with their tuberosities on top of the adjustable support elements. Ischial peak pressure mapping was used to monitor the ITs when positioning the subject in the chair and aligning the anatomical sagittal plane with the global $y-z$ plane. The footrests were set perpendicular to the seat and adjusted in a way that the thighs were fully supported by the seat except for the popliteal space. The experiment involved four cycles of downward sinusoid tuberal support adjustments within a range of $40 \mathrm{~mm}(-20 \pm 20 \mathrm{~mm})$ and a time period of 60 seconds. Seat reaction forces, buttock interface pressure, tuberal adjustment (D) and buttock tissue oxygenation $\left(\mathrm{SO}_{\mathrm{sk}}\right.$ and $\mathrm{SO} 2_{\mathrm{sc}}$ ) and perfusion $\left(\mathrm{BF}_{\mathrm{sk}}\right.$ and $\left.\mathrm{BF}_{\mathrm{sc}}\right)$ were captured and stored for offline analyses. Prior to the experiment, the initial values for all quantities were derived during a one-minute steady-state reference measurement with fully loaded ITs. Typical examples for D, $\mathrm{DI}_{\mathrm{f}}, \mathrm{DI}_{\mathrm{p}} \mathrm{CP}_{\mathrm{f}}, \mathrm{CP}_{\mathrm{p}}, \mathrm{SO} 2_{\mathrm{sk}}, \mathrm{BF}_{\mathrm{sk}}, \mathrm{SO} 2_{\mathrm{sc}}$ and $\mathrm{BF}_{\mathrm{sc}}$ during TSE adjustment are shown in figure 6.3A. 


\subsubsection{Statistics}

The initial values (init $\pm \mathrm{sd}$ ) and ranges $(\Delta \pm \mathrm{sd})$ for all quantities $\left(\mathrm{D}, \mathrm{DI}_{\mathrm{f}}, \mathrm{DI}_{\mathrm{p}}, \mathrm{CP}_{\mathrm{f}}\right.$, $\mathrm{CP}_{\mathrm{p}}, \mathrm{SO} 2_{\mathrm{sk}}, \mathrm{BF}_{\mathrm{sk}}, \mathrm{SO} 2_{\mathrm{sc}}$ and $\mathrm{BF}_{\mathrm{sc}}$ ) were derived and averaged over all subjects. To evaluate whether tuberal support adjustment is effective to regulate buttock load, all quantities were first normalized to its total range as shown in figure $6.3 \mathrm{~B}$ for $\mathrm{SO} 2_{\mathrm{sc}}$. Normalized quantities for buttock load $\left({ }^{n} \mathrm{DI}_{\mathrm{f}},{ }^{\mathrm{n} D I_{p}},{ }^{\mathrm{n}} \mathrm{CP}_{\mathrm{f}},{ }^{\mathrm{n}} \mathrm{CP}_{\mathrm{p}},{ }^{\mathrm{n}} \mathrm{SO} 2_{\mathrm{sk}}, \mathrm{BBF}_{\mathrm{sk}},{ }^{\mathrm{n} S O} 2_{\mathrm{sc}}\right.$ and ${ }^{n} \mathrm{BF}_{\mathrm{sc}}$ ) were then related to the normalised tuberal adjustment $\left({ }^{\mathrm{n}} \mathrm{D}\right)$. Linear relations were assumed and the coefficients of correlation (r) with accompanying significance (p) were derived. We then calculated the coefficients of determination $\left(r^{2}\right)$ which gives us an indication about the strength of the relation.

\subsection{Results}

During TSE adjustment, the initial values (initial $\pm \mathrm{sd}$ ) and ranges $(\Delta \pm \mathrm{sd}$ ) were computed for all quantities $\left(\mathrm{D}, \mathrm{DI}_{\mathrm{f}}, \mathrm{DI}_{\mathrm{p}}, \mathrm{CP}_{\mathrm{f}}, \mathrm{CP}_{\mathrm{p}}, \mathrm{SO} 2_{\mathrm{sk}}, \mathrm{BF}_{\mathrm{sk}}, \mathrm{SO} 2_{\mathrm{sc}}, \mathrm{BF}_{\mathrm{sc}}\right)$ and averaged over all subjects (Tab 6.1). Note that the number of trials is smaller for measurement of tissue oxygenation and perfusion $\left(\mathrm{SO}_{\mathrm{sk}}, \mathrm{BF}_{\mathrm{sk}}, \mathrm{SO} 2_{\mathrm{sc}}\right.$ and $\left.\mathrm{BF}_{\mathrm{sc}}\right)$. Five out of fifteen subjects showed no response in buttock tissue oxygenation and perfusion when adjusting the tuberal support elements (TSE). This group was excluded for analysis because the average values for $\mathrm{SO}_{\mathrm{sk}}, \mathrm{BF}_{\mathrm{sk}}, \mathrm{SO}_{\mathrm{sc}}$ or $\mathrm{BF}_{\mathrm{sc}}$ during the experiment were not significantly different than the initial values.

Normalized quantities for buttock load ( ${ }^{n} \mathrm{DI}_{\mathrm{f}},{ }^{\mathrm{n} D I} \mathrm{p},{ }^{\mathrm{n}} \mathrm{CP}_{\mathrm{f}},{ }^{\mathrm{n}} \mathrm{CP}_{\mathrm{p}},{ }^{\mathrm{n} S O} 2_{\mathrm{sk}}, \mathrm{BBF}_{\mathrm{sk}},{ }^{\mathrm{n} S O} 2_{\mathrm{sc}}$ and ${ }^{n} B_{s c}$ ) were related to the normalized TSE adjustment $\left({ }^{n} \mathrm{D}\right)$. Linear relations were assumed and the coefficients of correlation ( $\mathrm{r}$ ) with accompanying significance $(\mathrm{p})$ were derived and shown in table 6.1. Coefficients of determination $\left(\mathrm{r}^{2}\right)$ were calculated as an indication about the strength of the relation.

Table 6.1: Initial values (initial $\pm \mathrm{sd}$ ), ranges $(\Delta \pm \mathrm{sd}$ ), coefficient of correlation $(\mathrm{r})$ and coefficients of determination $\left(\mathrm{r}^{2}\right)$ for $\mathrm{D}, \mathrm{DI}_{\mathrm{f}}, \mathrm{DI}_{\mathrm{p}} \mathrm{CP}_{\mathrm{f}}, \mathrm{CP}_{\mathrm{p}}, \mathrm{SO}_{\mathrm{sk}}, \mathrm{BF}_{\mathrm{sk}}, \mathrm{SO}_{\mathrm{sc}}$ and $\mathrm{BF}_{\mathrm{sc}}$.

\begin{tabular}{|c|c|c|c|c|c|c|}
\hline & & init \pm sd & $\Delta \pm \mathrm{sd}$ & $\mathrm{r}$ & $\mathrm{r}^{2}$ & \\
\hline $\mathrm{D}$ & {$[\mathrm{mm}]$} & $0 \pm 0$ & $40 \pm 0$ & $1.00^{*}$ & 1.00 & \\
\hline $\mathrm{DI}_{\mathrm{f}}$ & {$[-]$} & $0.36 \pm 0.6$ & $0.36 \pm 0.6$ & $0.97^{*}$ & 0.94 & \\
\hline $\mathrm{DI}_{\mathrm{p}}$ & {$[-]$} & $0.22 \pm 0.2$ & $0.16 \pm 0.2$ & $0.98^{*}$ & 0.95 & \\
\hline $\mathrm{CP}_{\mathrm{f}}$ & {$[\mathrm{mm}]$} & $59 \pm 14$ & $20 \pm 2$ & $-0.96^{*}$ & 0.92 & \\
\hline $\mathrm{CP}_{\mathrm{p}}$ & {$[\mathrm{mm}]$} & $58 \pm 9$ & $11 \pm 2$ & $-0.88^{*}$ & 0.78 & $\mathrm{n}=15$ \\
\hline $\mathrm{SO} 2_{\text {sk }}$ & [\%] & $41 \pm 22$ & $42 \pm 27$ & $-0.73^{*}$ & 0.54 & \\
\hline $\mathrm{BF}_{\mathrm{sk}}$ & {$\left[\mathrm{au}^{1}\right]$} & $9 \pm 15$ & $98 \pm 63$ & $-0.74^{*}$ & 0.54 & \\
\hline $\mathrm{SO} 2_{\mathrm{sc}}$ & [\%] & $62 \pm 3$ & $9 \pm 5$ & $-0.86^{*}$ & 0.74 & \\
\hline $\mathrm{BF}_{\mathrm{sc}}$ & {$\left[\mathrm{au}^{1}\right]$} & $102 \pm 56$ & $323 \pm 160$ & $-0.80^{*}$ & 0.64 & $\mathrm{n}=10$ \\
\hline
\end{tabular}

"statistical significant correlation $(\mathrm{p}<0.05)$.

${ }^{1} \mathrm{au}=$ arbitrary unit 
In figure 6.4, the left graphs reflect ${ }^{n} \mathrm{DI}_{\mathrm{f}}$ and ${ }^{\mathrm{n}} \mathrm{DI}$ in ine. The right graphs show how ${ }^{n} I_{f}$ and ${ }^{n} D I_{p}$ are related to ${ }^{n} D$. Path-dependent hysteresis effects are shown for ${ }^{n D I}{ }_{f}$ when adjusting the TSE up- and downward. The inter-individual variability (errorbars) was larger for ${ }^{n} \mathrm{DI}_{\mathrm{p}}$ than for ${ }^{\mathrm{n} D I_{\mathrm{f}}}$

In figure 6.5, the left graphs reflect ${ }^{n} \mathrm{CP}_{\mathrm{f}}$ and ${ }^{\mathrm{n}} \mathrm{CP}_{\mathrm{p}}$ in time. The right graphs show how ${ }^{n} \mathrm{CP}_{\mathrm{f}}$ and ${ }^{\mathrm{n}} \mathrm{CP}_{\mathrm{p}}$ are related to ${ }^{\mathrm{n}} \mathrm{D}$. The inter-individual variability (error-bars) was larger for ${ }^{\mathrm{n}} \mathrm{CP}_{\mathrm{p}}$ than for ${ }^{\mathrm{n}} \mathrm{CP}_{\mathrm{f}}$. Similar to the effect on ${ }^{\mathrm{n} D I}$, hysteresis is also shown for ${ }^{\mathrm{n}} \mathrm{CP}_{\mathrm{f}}$. During the first and last ten seconds of the adjustment cycle $\left(-0.2<{ }^{\mathrm{n}} \mathrm{D}<0.0\right)$, the effect on ${ }^{n} \mathrm{CP}_{p}$ seems opposite to what was found during the rest of the cycle.

The effects of TSE adjustment $\left({ }^{\mathrm{n} D}\right)$ on levels of oxygenation and perfusion in the skin $\left({ }^{\mathrm{n} S O} 2_{\mathrm{sk}}\right.$ and $\left.{ }^{\mathrm{BBF}_{\mathrm{sk}}}\right)$ and subcutaneous $\left({ }^{\mathrm{n} S O} 2_{\mathrm{sc}}\right.$ and $\left.{ }^{{ }^{\mathrm{BBF}}}{ }_{\mathrm{sc}}\right)$ subtuberal buttock tissue are shown in figure 6.6. All normalised quantities of blood supply were inversely related to ${ }^{\mathrm{n}} \mathrm{D}$. Variability between subjects is indicated with error bars.

\subsection{Discussion}

This study investigated the redistribution of external buttock load in relation to the pattern (i.e. dynamics) of subtuberal blood supply in sitting with a dynamic tuberal support of $1 / 60 \mathrm{~Hz}(80 \mathrm{~mm} / \mathrm{min})$.

\section{loaded}
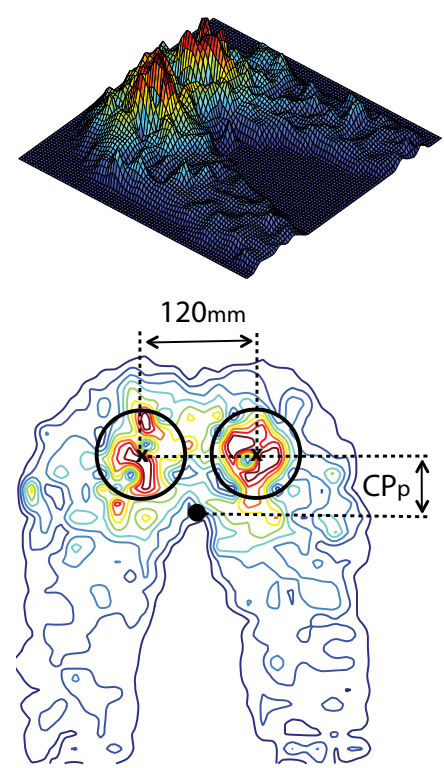

relieved

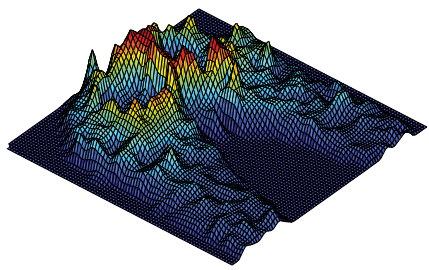

TSA

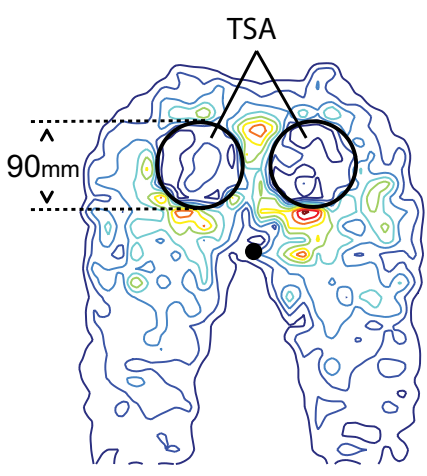

Figure 6.2: Buttock interface pressure distribution as measured with the external pressure mapping device (PMD) in loaded and relieved seating conditions. Spaced according to an intertuberal distance of $120 \mathrm{~mm}$, two areas for tuberal support (TSA) are defined on top of the round (Ø 90mm) adjustable tuberal support elements (TSE). Centre of pressure $\left(\mathrm{CP}_{\mathrm{p}}\right)$ was computed in forward direction relative to the middle of the TSA. 


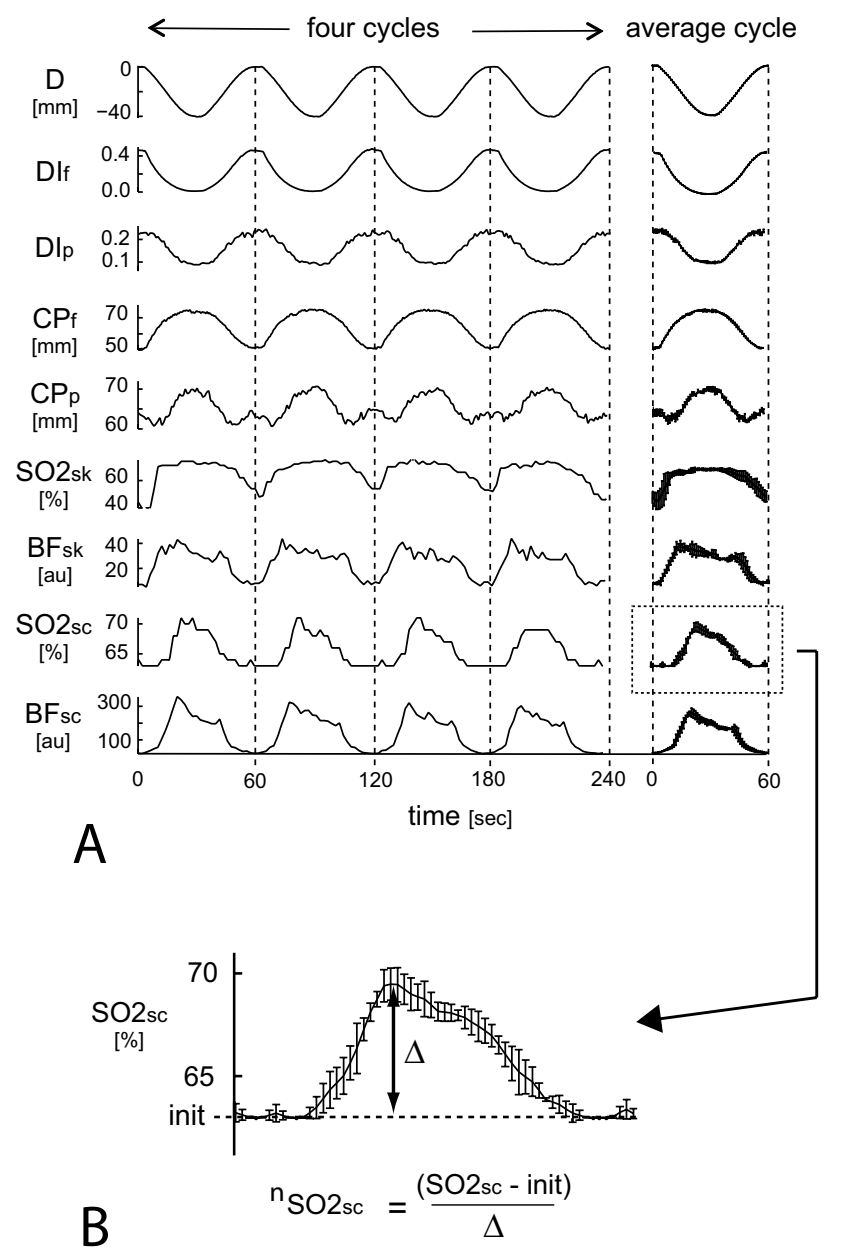

Figure 6.3: A: Typical examples for measurement of $\mathrm{D}, \mathrm{DI}_{\mathrm{f}}, \mathrm{DI}_{\mathrm{p}} \mathrm{CP}_{\mathrm{f}}, \mathrm{CP}_{\mathrm{p}}, \mathrm{SO}_{\mathrm{sk}}, \mathrm{BF}_{\mathrm{sk}}, \mathrm{SO} 2_{\mathrm{sc}}$ and $\mathrm{BF}_{\mathrm{sc}}$ when adjusting the TSE within a range of $40 \mathrm{~mm}$ and a time period of 60 seconds. $\mathrm{B}$ : All quantities were normalised as shown for $\mathrm{SO}_{\mathrm{sc}^{*}}$

When adjusting the TSE below the seating surface, significant effects were found on the contact load under the ITs, centre of buttock pressure (CP) and the subtuberal buttock tissue oxygenation $\left(\mathrm{SO}_{\mathrm{sk}}\right.$ and $\left.\mathrm{SO} 2_{\mathrm{sc}}\right)$ and perfusion $\left(\mathrm{BF}_{\mathrm{sk}}\right.$ and $\left.\mathrm{BF}_{\mathrm{sc}}\right)$. Downward TSE adjustment relieved the external tuberal load almost completely and shifted CP in forward direction. High coefficients of determination $\left(r^{2}>0.7\right)$ were found for DI and $\mathrm{CP}$ which indicate a strong relation with the level of tuberal support and suggested that the external ischial buttock load is regulable from TSE adjustment.

For most subjects, the level of oxygenation and perfusion in the subtuberal buttock tissue increased significantly when unloading the TSE.

However, weak coefficients of determination $\left(\mathrm{r}^{2}<0.7\right)$ indicates large variability between subjects. Individual differences in the dynamics/response of blood supply during support surface manipulations make precise regulation of skin and subcutaneous 
tissue oxygenation and perfusion difficult.

Differences in external buttock load as measured with the conventional PMD and the FSP could be ascribed to inaccuracies in PMD measurement under fully loaded seating conditions. When the TSEs were level with the seating surface, very high forces exerted on the pressure elements under the ITs. We observed that some elements could not resist such high contact loads and started saturating or even fail measuring. Due to this phenomenon, the tuberal interface pressure as measured with PMD was underestimated. The pressure elements of PMD were connected flexibly to minimize hammocking (Casey et al., 2001). However, buttock load did not relieve entirely under the ITs when the TSE were lowered. Limited flexibility of PMD caused that little tuberal pressure was maintained at all times.

Because the PMD was placed on top of the FSP, the latter measured entire tuberal pressure relief since the TSE completely lost contact with the buttock/PMD interface. Hammocking at the seat interface when the ITs were unloaded and inaccurate peak pressure readings when the tuberosities were fully loaded explain the differences in measurement between $\mathrm{CP}_{\mathrm{f}}$ and $\mathrm{CP}_{\mathrm{p}}$ and between $\mathrm{DI}_{\mathrm{f}}$ and $\mathrm{DI}_{\mathrm{p}}$ as shown in table 6.1.

Some interesting differences in measurement with the PMD and FSP were also observed during TSE adjustment. First, large variability (error-bars) was measured for ${ }^{n} \mathrm{DI}_{\mathrm{p}}$ and ${ }^{\mathrm{n}} \mathrm{CP}_{\mathrm{p}}$, which suggested that the reproducibility for PMD measurement was less than for the FSP. Second, path-dependent hysteresis for ${ }^{n} \mathrm{DI}_{\mathrm{f}}$ and ${ }^{\mathrm{n}} \mathrm{CP}_{\mathrm{f}}$ was observed when adjusting the TSE. Because the force elements of the FSP were located underneath the seating cushion, we ascribed these effects to friction between the seating cushion and the TSE. In loaded seating conditions, the cushions on top of the TSE expanded sideways and contacted the surrounding seating cushion. Although the cushions on the TSE were designed not to contact the surrounding cushion in loaded seating conditions, small levels of friction still occurred when adjusting the TSE up- and downward. Third, when adjusting the TSE just below the seating surface, the PMD did not monitor pressure change on the TSE due to saturation and failing of some pressure elements that were located directly underneath the ITs. As a result, $\mathrm{CP}_{\mathrm{p}}$ did not shift forward in the first stage of tuberal unloading. This phenomenon is less profound for DI because the dispersion index is not an absolute measure but expresses the tuberal interface pressure relative to the total buttock interface pressure.

Reliability problems when quantifying buttock load with absolute peak pressure measurement have also been reported in other studies (Sprigle et al., 2003, Stinson et al., 2002). Relative measures have therefore been developed that expresses absolute peak pressures relative to the total buttock pressure. Because better testretest reliabilities have been reported, it is often proposed to use relative measures to quantify pressure distribution (Sprigle et al., 2003, Staarink, 1995). Despite using relative measures, our findings suggest that hammocking at the seat interface and inaccurate peak pressure measurement could still lead to misinterpretation of the buttock interface pressure distribution.

Because the external buttock load does not provide direct information about the internal tissue conditions, additional measurement of the ischial blood supply has been suggested as being useful to evaluate the viability of internal buttock tissue (Black et al., 2007). However, clinical evaluation of the microcirculation in the subcutaneous tissue is difficult and most available measuring techniques (e.g. laser 

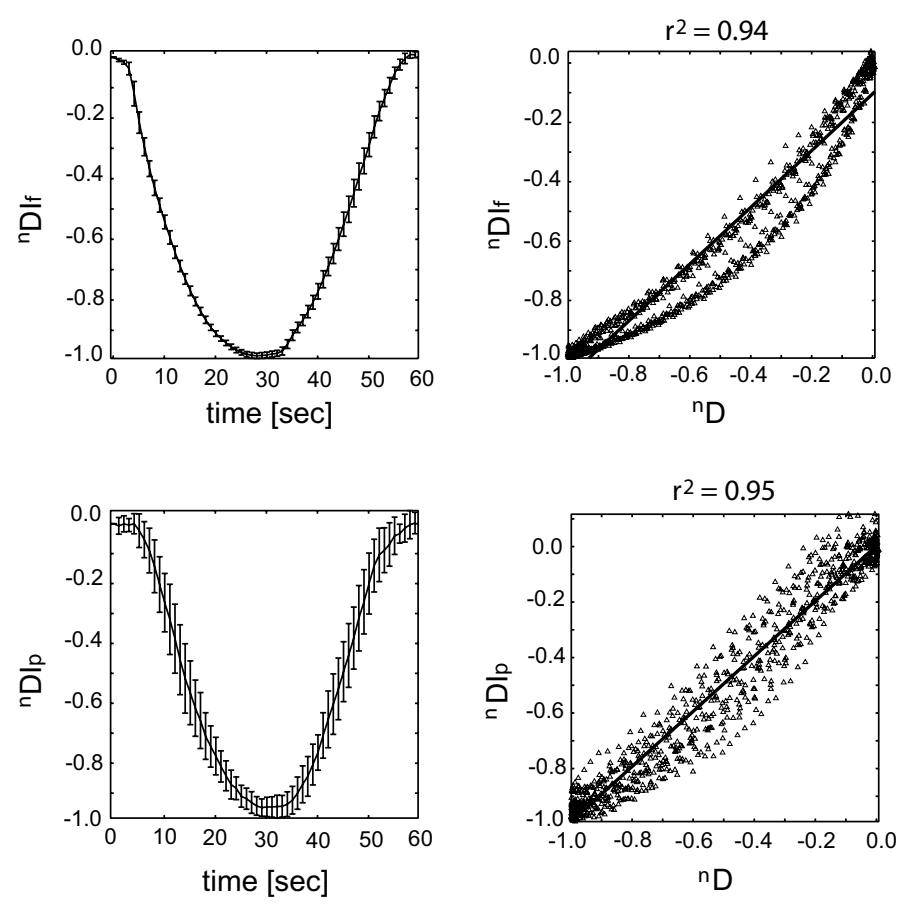

Figure 6.4: Left: ${ }^{n} \mathrm{DI}_{\mathrm{f}}$ and ${ }^{\mathrm{n} D I_{p}}$ in time. Right: ${ }^{\mathrm{n}} \mathrm{DI}_{\mathrm{f}}$ and ${ }^{\mathrm{n} D I_{p}}$ related to ${ }^{\mathrm{n}} \mathrm{D}$. Linear relations were assumed and coefficients of determination $\left(\mathrm{r}^{2}\right)$ were derived.

Doppler flowmetry, transcutaneous oxygen tension, tissue reflectance spectrometry and thermography) struggle with technical limitations concerning measuring depth and reliability problems. Light-guide tissue spectrophotometry is a relatively new technique to evaluate micro-vascular tissue perfusion and has been developed to facilitate observation of tissue viability during surgery (Holzle et al., 2006, Holzle et al., 2003). The effectiveness of this technique has also been demonstrated in early identification and quantification of tissue ischemia in diabetic foot ulcers (Rajbhandari et al., 1999, Beckert et al., 2004). Most diagnostic devices that measure tissue perfusion and oxygenation are limited to a measuring depth of 1 or 2 millimetres, which makes that most studies focus on the effects of external buttock load on tissue oxygenation and perfusion of the skin only. The device that was used in the present study could monitor tissue oxygenation and perfusion up to a measuring depth of approximately $8 \mathrm{~mm}$. This provides more insight in tissue regions closer to places of interests than what normally could be reached.

For 10 out of all 15 subjects we found that oxygenation and perfusion were inversely related to the applied loads under the ITs and that the perfusion and oxygenation values of the skin were more sensitive to the induced tuberal adjustments than what was measured in the subcutaneous buttock tissue. Because the induced tissue deformations are better distributed when travelling into deeper tissue regions, less internal stress/strain fluctuations are expected in comparison to the skin. This rather limited effect on the internal mechanical tissue properties indicates that the initial 

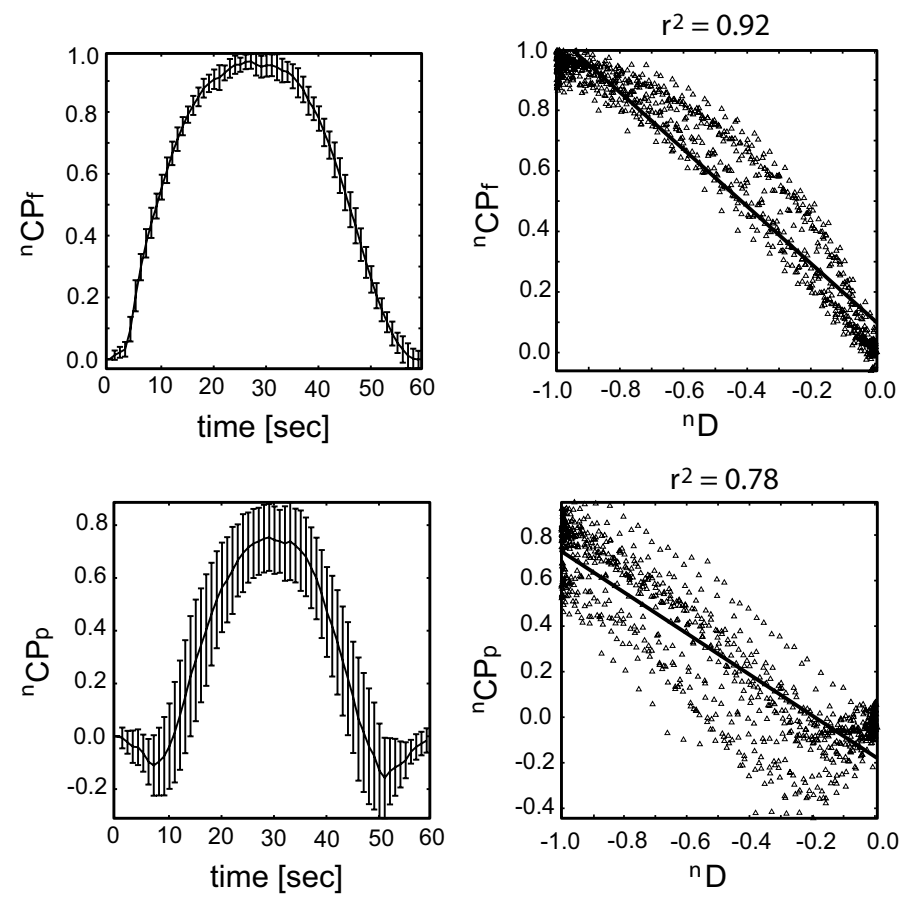

Figure 6.5: Left: ${ }^{\mathrm{n}} \mathrm{CP}_{\mathrm{f}}$ and ${ }^{\mathrm{n}} \mathrm{CP}$ in time. Right: ${ }^{\mathrm{n}} \mathrm{CP}_{\mathrm{f}}$ and ${ }^{\mathrm{n}} \mathrm{CP}_{\mathrm{p}}$ related to ${ }^{\mathrm{n}} \mathrm{D}$. Linear relations were assumed and coefficients of determination $\left(\mathrm{r}^{2}\right)$ were derived.

seating conditions are of great importance. When the initial shear force and pressure gradients within the tissue are relatively high from the start, and the subtuberal buttock tissue already involves questionable levels of blood supply, it is uncertain whether periodic pressure relief allows full recovery of the ischial buttock tissue after prolonged deformation. In some subjects we even observed that the flow of blood in the microcirculation of the skin completely stagnated, which indicated that the superficial arteries entirely collapsed. Interference of the probes in the tuberal zone probable intensified this effect. Future experiments must evaluate whether it is useful to surround the probes with a (silicon) mold shaped around the buttock tissue under the ITs. This way, the contact load equally distributes over the ITs and less interference with the ischial buttock tissue is expected.

Five subjects showed no significant response in buttock tissue perfusion and oxygenation, and were classified as non-responding subjects. We speculated about this phenomenon in our previous study (Reenalda et al., 2009), in which we referred to a study of Bader (Bader, 1990) who found similar non-responding patterns when investigating the effects of dynamic cushions on skin perfusion in healthy and impaired subjects. Bader reported that the non-responding group might be subjected to an increased risk for tissue ischemia due to a potential impaired physiological control mechanism. However, in our studies the opposite might be true since the initial levels of tissue perfusion involve higher values for the non-responding group compared to the responding group. This could indicate that the non-responders have less risk for deep tissue injury because they already experience relatively good 

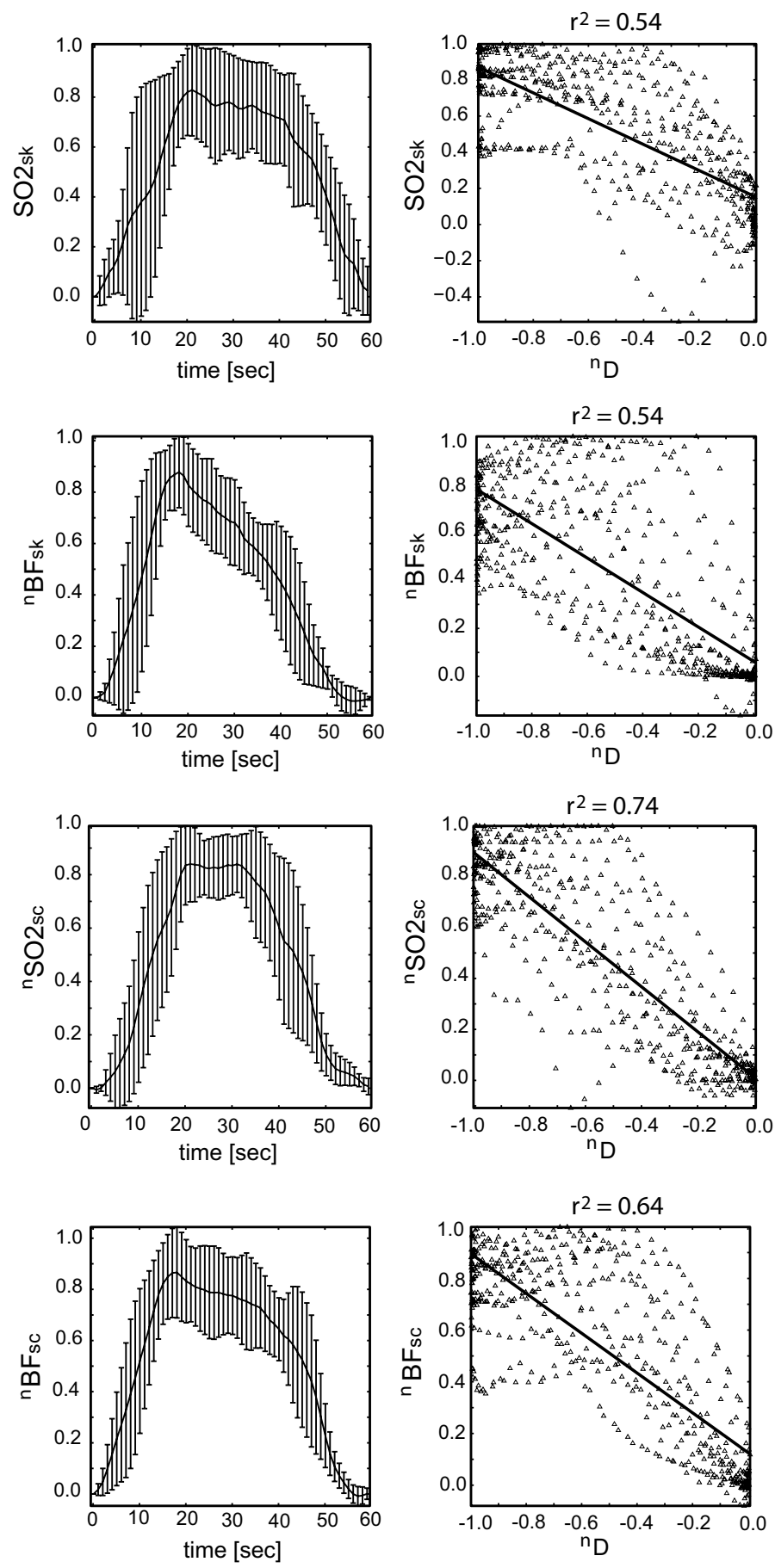

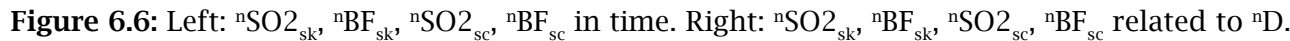
Linear relations were assumed and coefficients of determination $\left(\mathrm{r}^{2}\right)$ were derived. 
perfusion levels. Their physiological control mechanism potentially allows them to maintain blood flow, even when the ischial buttock tissue is fully loaded. This could benefit the tissue viability and decreases the risk for pressure ulcer formation in continuous static sitting.

Another explanation could be that the probes simply did not measure the area where the most deformation occurred. When the area of blood flow stagnation shifted to tissue regions just beyond the reach of the measurement probes, no effect on oxygenation and perfusion levels will be found.

Future studies must address more attention to this matter. In-depth investigation about the physiological effects of tissue loading on ischial blood supply in healthy subjects and in impaired subjects with high and low risk for PU might reveal whether the differences in blood pattern is linked to differences in the physiological mechanism that controls the supply of blood in the buttock tissue in loaded and unloaded seating conditions.

For all responding subjects, the response in tissue oxygenation and perfusion could roughly be divided into three stages (Fig. 6.6). Strong hyperaemia effects were observed in the first stage. Unloading the ITs induced significant increase in skin and subcutaneous tissue oxygenation and perfusion. A moderate overshoot in blood flow occurs due to temporary ischemia and build-up blood pressure from preceding tissue deformation. The second stage showed that from a certain point, downward tuberal adjustments were no longer accompanied with an increase in blood supply. We even observed a gradual outflow of blood which we ascribe to the overshoot in the beginning of the second stage. The last (third) stage represents a strong decrease in oxygenation and perfusion when reloading the ITs.

Upward TSE adjustments were accompanied with a strong outflow of blood which was caused by occlusion of blood vessels when the buttock load under the ITs gradually increased. Rapid inflow of blood in the initial stage of tuberal unloading followed by a more gradual outflow in the rest of the movement cycle indicates that the average blood supply increases when the adjustment frequency increases. This might explain the outcomes of our previous study (Reenalda et al., 2009), which showed higher average values for skin oxygenation and subcutaneous tissue perfusion in seating conditions with higher adjustment frequencies.

We must take in mind that ischemia-reperfusion injury can occur from the reperfusion of blood to previously ischemic tissue (Tsuji et al., 2005, Peirce et al., 2000). During prolonged ischemia, the depletion of oxygen changes the metabolism from aerobic to anaerobic. As a result, the concentration of glucose reduces and tissue acidification occurs due to the accumulation of lactic acid (Stekelenburg et al., 2008). Reperfusion of oxygen-derived free-radicals into the hypoxic acidified tissue after unloading, potentially initiate a cascade of harmful events that could result in tissue necrosis. However, we do not expect that this will happen during periodic TSE adjustment of $80 \mathrm{~mm} / \mathrm{min}$, since the time of ischemia is not long enough to completely deprive the tissue from its oxygen and to acidify the interstitial fluid. This way, the tissue remains invulnerable for the inflow of free-radical and less damage is expected.

The composition of the cushion on the TSE was slightly different compared to the rest of the support surface. This was done because the stiffness of the support surface alters when cutting out two round holes. Due to the loss of surface tension, the stiffness of the cushion reduces when coming closer to the cut-outs. The stiffness 
on the TSEs reduces even more due to the complete loss of surface tension. We therefore modified the TSE upholstering and slightly increased its stiffness. This gave the impression of sitting on a hard surface when the TSE were level with the seating surface. This especially applied for individuals who were thin and had less ischial buttock tissue to distribute the interface pressure over a larger contact area. Depending on the mechanical characteristics of the support cushion, it has been reported that the peak pressures under the ITs could be $30 \%$ higher in thin (BMI $<20$ $\mathrm{kg} / \mathrm{m}^{2}$ ) individuals than in 'normal' (BMI 20-25 kg/m²) individuals (Kernozek et al., 2002). Although less profound, we also observed some influence of body build on buttock pressure distribution. Under fully loaded seating conditions we found that for individuals with a thin body type the TSE supported up to $40 \%$ of all seating load. When such high forces are locally exerted on the buttock tissue under the tuberosities, significant deformation of the skin and subcutaneous tissue is expected. As a consequence, we found that for some individuals the supply of blood completely stagnated and the level of skin oxygenation and perfusion decreased to zero. Such extreme seating conditions, in which ischemia is combined with excessive internal tissue deformation due to high external load, must be prevented at all times because it aggravates the extent and onset of tissue damage within very short periods of exposure (Stekelenburg et al., 2008).

During downward TSE adjustment, the contact load is relieved under the ITs and shifted towards the edges of the cushion cut-outs where it exerted on the surrounding tissue regions such as the thighs and sacrum. That TSE adjustment not only affects the buttock tissue under the ITs is clearly shown in figure 6.2. During the unloading phase, high shear forces and pressure gradients were introduced in the tissue around the ITs. Adjustment of the TSE must therefore be applied periodically to prevent tissue damage in the surrounding tissue as well.

It is very hard to predict how long the ischial buttock tissue can sustain certain deformation before tissue degeneration takes place, and how often the TSE must be adjusted to maintain tissue viability in prolonged static sitting. Several researchers have taken much trouble to identify reliable thresholds for minimal pressure and duration which consistently generates tissue necrosis (Bouten et al., 2003, LinderGanz et al., 2006, Stekelenburg et al., 2008). However, no reliable and clinically applicable indicator has yet been found that predicts the risk for PU from continuous deformation. Until this has been revealed, the US Department of Health recommends individuals in a wheelchair to relieve buttock pressure every 15 minutes (Agency for Health Care Policy and Research, 1994). Whether it is sufficient to maintain adequate buttock tissue viability in prolonged static wheelchair sitting by adjusting the TSE every 15 minutes is something we have to evaluate.

\subsection{Conclusions}

TSE adjustment seemed effective to regulate centre of buttock pressure and the dispersion index under the ITs. Differences in measurement with the FSP and PMD have been found. Hammocking at the seat interface and inaccurate peak pressure readings could misinterpret pressure distribution as measured with the PMD.

Blood supply to the subtuberal buttock tissue was inversely related to the applied loads under the ITs. A rapid inflow of blood in the initial stage of tuberal unloading 
followed by a more gradual outflow in the rest of the movement cycle indicates that the average blood supply increases when the adjustment frequency increases.

Furthermore, TSE adjustment not only affected the buttock tissue under the ITs but also the tissue under the sacrum and thighs. This suggests a potential benefit for buttock tissue viability under the whole ischial region.

How TSE adjustment influences external buttock load and the dynamics of blood supply to the (subtuberal) buttock tissue in impaired individuals is still to be investigated.

\subsection{Acknowledgements}

This study was partly funded by the Dutch Ministry of Economical Affairs, SenterNovem, grant TSIT3043. The authors would like to thank the engineering company Demcon (Oldenzaal, the Netherlands) for developing the experimental simulator chair. 


\section{Chapter 7}

\section{Decoupled Pelvis Rotations to Regulate Low Back Load}

Resubmitted as: P. van Geffen, J. Reenalda, P.H. Veltink and H.F.J.M. Koopman. Decoupled pelvis adjustment to induce lumbar motion: a technique that controls low back load in sitting (under revision) 


\section{Abstract}

Static sitting in confined settings have been associated with low back pain in sedentary occupations such as office works and car driving. To prevent lumbar discomfort in prolonged static sitting, periodic motion of the lumbar spine is needed. Because the pelvis forms the basis for lumbar spine curvature, we developed a passive motion technique that adjusts pelvis orientation independent from the trunk. This study uses a computer-aided simulator chair instrumented with a mechanism for decoupled pelvis adjustment and investigates the effects on low back load in eighteen healthy male individuals.

Experiments involved measurement of body segments orientation, interface back pressure distribution and seat reaction forces during quasi-static actuated pelvis rotation in the sagittal and frontal plane. Joint forces and torques were estimated in the lumbosacral spine region.

In sagittal pelvis adjustment, the pelvis rotated $19 \pm 3$ degrees and strong relations $\left(r^{2}>0.8\right)$ were found with centre of back pressure in upward direction $\left(c p_{z}, r^{2}=0.90\right)$, lumbosacral shear force in forward direction $\left(F_{l y}, r^{2}=0.91\right)$, lumbosacral normal force in axial direction $\left(F_{l z}, r^{2}=0.80\right)$, lumbosacral torque around lateral direction $\left(T_{l x}, r^{2}=0.80\right)$ and interface pressure at the lower back $\left(P_{l b}, r^{2}=0.88\right)$. In frontal pelvis adjustment, the pelvis rotated $9 \pm 1$ degrees and strong relations were found with centre of back pressure in lateral direction ( $\left.c p_{x}, r^{2}=0.90\right)$, lumbosacral torque around forward direction $\left(T_{l y}\right.$, $\left.r^{2}=0.98\right)$ and lumbosacral torque around axial direction $\left(T_{l z}, r^{2}=0.95\right)$.

These findings indicate that decoupled pelvis adjustment could be an applicable intervention to control low back load in prolonged static sitting. 


\subsection{Introduction}

Pain disorders at the lower back are a worldwide concern in sedentary occupations such as office works, vehicle driving and dealing with heavy industrial equipment. Long-term sitting in confined settings have been associated with an increased risk for low back pain (LBP) (Todd et al., 2007). Although no clear epidemiological proof exists, literature reveals that prolonged sitting in awkward postures and in combination with exposure to whole body vibration (WBV) potentially facilitates the process for low back discomfort (Lis et al., 2007). Therefore, it might be beneficial to deal with risk factors other than just sitting itself. However, the job conditions do not always allow reducing the time to exposure or the intensity of WBV. Instead, it is preferred to focus on seating interventions that prevent awkward postures and control the mechanical stress levels at the lower back.

There is evidence that the sensation of discomfort in persons who suffer from LBP reduces when sitting with a lordotic spinal posture (Williams et al., 1991). However, many persons adopt a flexed spinal curvature with posterior tilted pelvis while doing sedentary work. Such awkward spinal postures have often been associated with aggravation of back discomfort due to a reduction of back muscle activity (Callaghan and Dunk, 2002, Mork and Westgaard, 2009, O'Sullivan et al., 2006). This so-called flexion-relaxation phenomenon is characterized by a transition of spine stabilisation from active trunk muscles to passive spine structures such as the posterior ligaments and intervertebral discs. Continuous deformation of these structures will eventually change the passive spine characteristics and potentially increase the risk for injury at the lower back (Beach et al., 2005, Parkinson et al., 2004).

Beach et al. (Beach et al., 2005) found that the passive flexion stiffness of the lumbar spine increases within the first two hours of sitting. This demonstrates that spinal tissue characteristics already change after relatively short periods of exposure. They associated this stiffness increase with an increased passive resistance of back muscles, which they assumed being the primary flexion-resisting tissues. Beach et al. (Beach et al., 2005) and Parkinson et al. (Parkinson et al., 2004) reported that these findings suggest an increased risk for low-back injury when individuals perform full lumbar flexion tasks after prolonged sitting with a flexed lumbar posture.

Continuous stress at the lower back will eventually cause that the stiffness of the posterior spine ligaments and intervertebral discs decrease due to visco-elastic creep (Keller et al., 1987, Argoubi and Shirazi-Adl, 1996, Hedman and Fernie, 1997). Sustained mechanical spinal loading without significant durations of stress relief causes nutrient supply to stagnate and load bearing spinal structures to degenerate (Urban et al., 2004). Because the intervertebral discs and facet joints are important structures to maintain segmental stability, degenerative processes in these structures change the segment motion characteristics associated with spinal instability and low back injury (Fujiwara et al., 2000a, Fujiwara et al., 2000b).

With the assumption that body movement is needed to prevent discomfort in prolonged sitting, Van Dieën et al. (van Dieen et al., 2001) investigated the beneficial effects of a dynamic office chair compared to a chair with fixed seat and backrest. They found that spinal shrinkage reduced when using a chair with dynamic body support. This suggests that dynamic chairs offer a potential advantage with respect to trunk loading and the prevention of lumbar discomfort. 
Based on the above-mentioned considerations, it seems that a lordotic lumbar spine curvature together with significant levels of spinal movement are needed to facilitate the perfusion of intervertebral disc nutrition (Holm and Nachemson, 1983) and to decrease the risk for disc degeneration (Urban et al., 2004). To reduce incidence of low back injury in individuals that undergo insufficient postural variation, several techniques have been reported that enhance lumbar motion and relieve static loads associated with spinal tissue degeneration and pain (van Deursen et al., 1999, Aota et al., 2007, Reinecke et al., 1994).

Van Deursen et al. (van Deursen et al., 1999) described an ergonomic chair that uses passive motion for continuous axial pelvis rotation imposed by extremely small rotational seat movements in the horizontal plane. Based on subjective measures for lumbar discomfort, they reported a positive effect on pain relief in subjects suffering from low back injury. Although they never measured the mechanical or physiological effects on the internal spine conditions, they explained this reduced sensation of discomfort to improved processes of osmosis and diffusion for nutrient to the intervertebral discs. To find proof for the efficacy on pain relief, Lengsfeld et al. (Lengsfeld et al., 2007) subjected this ergonomic chair to a rigorously designed randomized multicenter study under 280 office-workers suffering from LBP. After two years of observations, no significant difference was found when comparing the effects on lumbar comfort for the ergonomic chair and other high quality officechairs. They attributed this lack of significant results to the minimal degree of axial rotation amplitude and they suggested that considerable higher rotation amplitudes might provide better outcomes with regard to lumbar pain relief.

Contrary to the concept of induced axial spinal rotations, some techniques have been reported that affects spine curvature in the sagittal plane. Reinecke et al. (Reinecke et al., 1994) described a commercially available device (Backcycler, ergonomics Inc., Winooski, VT) that induces continuous passive motions to the lower spine by an alternating inflatable lumbar support. Based on subjective measures for low back discomfort, they reported an improved tolerance for prolonged sitting. Viswanathan and co-authors (Viswanathana et al., 2006) tested the beneficial effects on musculoskeletal discomfort in operators of heavy earth-moving equipment. Seating discomfort usually increases during the day when dealing with persistent exposure to WBV. However, when using the inflatable lumbar support no difference was found between the early morning and evening. This indicates that the dynamic lumbar support improves tolerance to WBV when doing industrial work with heavy earthmoving equipment. Beach et al. (Beach et al., 2003) and Atoa et al. (Aota et al., 2007) also investigated the effects of this adjustable lumbar support in prolonged sitting. Beach et al. (Beach et al., 2003) found no differences in activation patterns of the lower erector spinae muscle, and suggested that the potential benefits for pain relief might be caused by the facilitation of the intervertebral disc nutrient. Atoa et al. (Aota et al., 2007) did also not observed any statistical evidence for a reduced feeling of lumbar discomfort. However, they observed that the induced lumbar motion was less than that reported by Reinecke et al. (Reinecke et al., 1994), which might explain why no positive effect was found. Adjustments of the lumbar support that induce a more profound effect on lumbar motion might increase the beneficial effect on lumbar pain relief in sitting.

Because the pelvis directly affects the curvature of the lumbar spine, pelvis angle could 
play a key role in the assessment of spinal posture and load. Based on measurement of back muscle activity, this assumption is supported by findings of O'Sullivan (O’Sullivan et al., 2006) and Mork and Westgaard (Mork and Westgaard, 2009). Both studies reported a great influence of pelvis posture on back muscle activity. They found that muscle activity increases in anterior tilted pelvis postures, and reduces in posterior tilted pelvis postures. The implementation of a dynamic back support that periodically adjusts the adopted pelvis posture might therefore prevent continuous muscle activation or deactivation and potentially controls the applied loads at the lower back.

With this purpose, we designed and built a seating intervention that rotates the pelvis in frontal and sagittal direction independent from the orientation of the trunk and seat support. In previous studies we already demonstrated the beneficial effects on postural alignment (van Geffen et al., 2008) and the control of buttock load associated with pressure ulcer development (van Geffen et al., 2009). However, the potential benefit of this novel technique on the control of spinal load has not yet been investigated.

Based on objective measures for low back load, the present study evaluates whether so-called decoupled pelvis adjustment is applicable to control the net lumbosacral 'joint' forces and torques in sitting. Because periodic pelvis adjustment will induce significant lumbar motion if the orientation of the upper body does not change, we expect great influence on the mechanical stress levels at the lower back. This could be in favour of preventing lumbar discomfort and improving tolerance to prolonged static sitting.

\subsection{Methods}

Part of the experimental setup, protocol en analysis were similar to some of our previous work, which focussed on the control of body posture (van Geffen et al., 2008) en pressure ulcer prevention (van Geffen et al., 2009).

\subsubsection{Subjects}

Eighteen $(n=18)$ healthy male subjects with no clinical history of low back pain were recruited for this study. Mean (+sd) anthropometric subject data is presented in table 7.1. All subjects read and signed an 'informed consent', which explained the objective and experimental protocol. This study was approved by the Committee for Medical Ethics of Roessingh Rehabilitation Centre (Enschede, the Netherlands).

Table 7.1: Anthropometric subject data

\begin{tabular}{lllll}
$\mathrm{n}=18$ & age [years] & weight $[\mathrm{kg}]$ & height $[\mathrm{m}]$ & BMI $\left[\mathrm{kg} / \mathrm{m}^{2}\right]$ \\
\hline mean & 22.6 & 74.9 & 1.84 & 22.1 \\
sd & 2.4 & 8.0 & 0.05 & 1.9 \\
BMI = Body Mass Index & & &
\end{tabular}




\subsubsection{Principle for Decoupled Pelvis Adjustment}

Figure 7.1 shows the principle that adjusted the pelvis in sagittal direction independent from the trunk and seat support. It was based on a parallelogram design that placed the axes for trunk and pelvis rotation within the lumbar region and under the ischial tuberosities (ITs) respectively. As shown, the backrest was fixed on the upper link of the parallelogram. Actuation of the parallelogram configuration imposed sagittal pelvis rotation without affecting the orientation of the trunk.

To adjust the pelvis sideways, the seat support was divided into a left and right seat part. Both parts were oppositely adjustable in height. Actuation of the relative height between the left and right seat part rotated the pelvis in the frontal plane around an axis between the ITs.

\subsubsection{Experimental Setup}

Experiments were performed with a adjustable simulator chair that was instrumented with the concept for decoupled pelvis rotation (Fig. 7.2).

The backrest was mounted on a sledge to allow vertical trunk translations and to align the pelvis with the parallelogram. The weight of the backrest was counterbalanced. The backrest, seat, parallelogram and footrests were adjustable to align body posture with chair configuration.

For accurate three-dimensional measurement of seat reaction forces, six uni-axial load cells (FUTEK ${ }^{\circledR}$, California, USA) were mounted in the seat frame. A pressure mapping device (Tekscan ${ }^{\circledR}$ Comformat, Boston, USA) was placed over the backrest to measure back pressure distribution. The device consisted of 1024 (32 x 32) rectangular pressure elements of $15 \mathrm{~mm} \times 15 \mathrm{~mm}$ and was calibrated with an upper limit of $33 \mathrm{kPa}(250 \mathrm{mmHg})$.

Reflective markers were placed on the chair and selected anatomical landmarks. Three-dimensional chair configuration and body segment orientations were obtained using an infrared camera motion capturing system (VICON ${ }^{\circledR}$, Oxford, UK). To prevent skin artifacts during pelvis movement, a pelvis mold (PM) was shaped around the
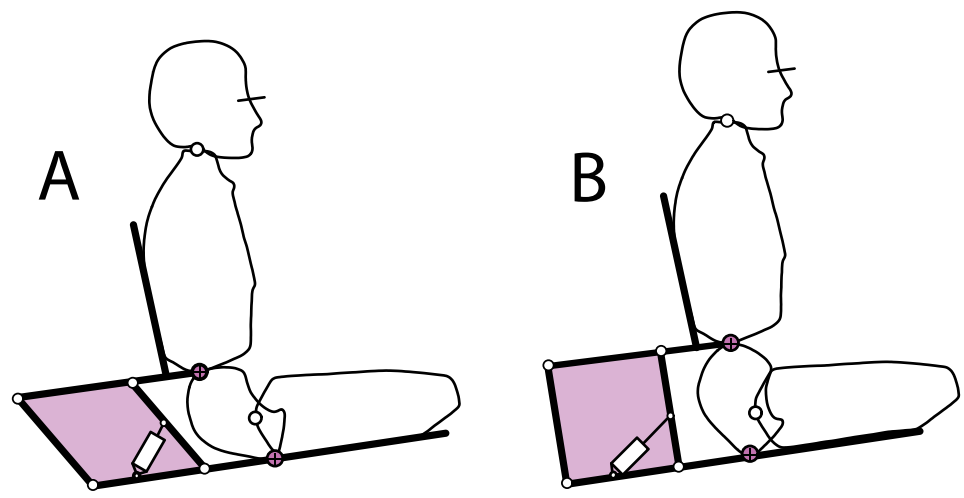

Figure 7.1: Principle for decoupled pelvis adjustment in the sagittal plane. The backrest is fixed on a parallelogram that rotates the pelvis around a pivot under the ITs when adjusting its configuration. A: posterior pelvis rotation. B: anterior pelvis rotation 
left and right lateral iliac crest and clamped the Anterior Superior Iliac Spine and Posterior Superior Iliac Spines for optimal fixation (Fig. 7.3). To prevent problems with pelvis marker visibility, an inertial sensor (MT ${ }^{\circledR}$, Xsens, Enschede, the Netherlands) was attached on the PM as an alternative to estimate pelvis orientation. The line of gravity was used to derive sensor inclination that we related to the orientation of the $\mathrm{PM}$ as shown in figure 7.3.
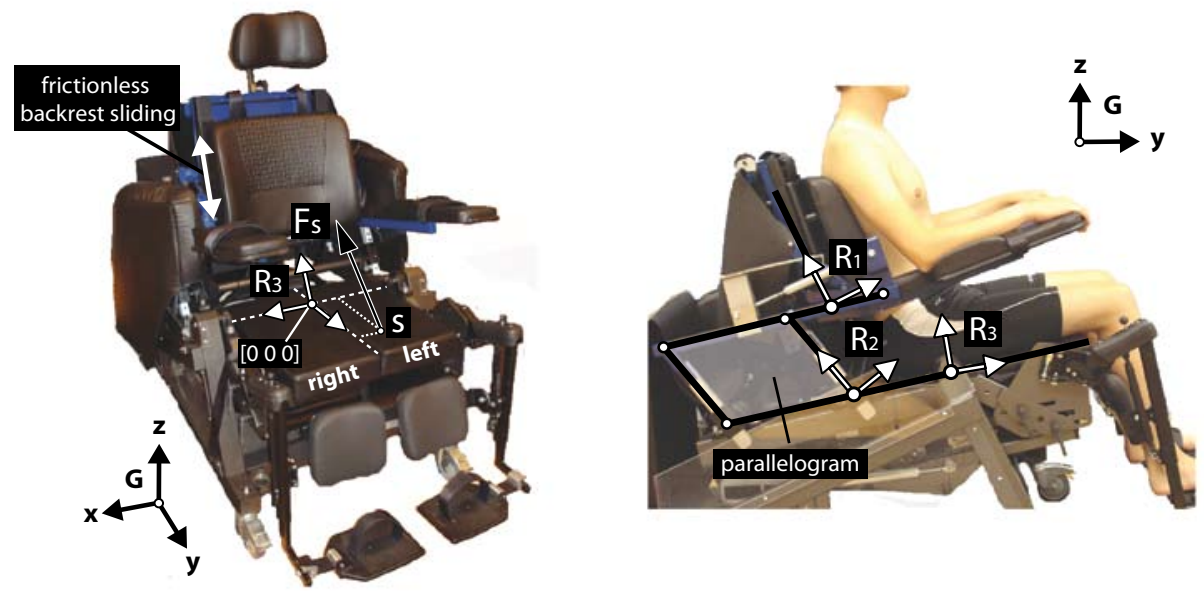

Figure 7.2: Experimental setup. Computer-aided adjustable simulator chair with the concept for decoupled pelvis adjustment. The configuration of the parallelogram and the height between the left and right seat parts are adjustable to rotate the pelvis in the sagittal and frontal plane respectively. Six uni-axial load cells are mounted in the seat to measure seat reaction force $\left(\mathrm{F}_{\mathrm{s}}\right)$ and its point of application (S) relative to $R_{3}$ The origin lies in $\left[\begin{array}{lll}0 & 0 & 0\end{array}\right] . R_{1}, R_{2}$ and $R_{3}$ define the local coordinate frames for the backrest, parallelogram and seat. $G$ defines the global reference frame.
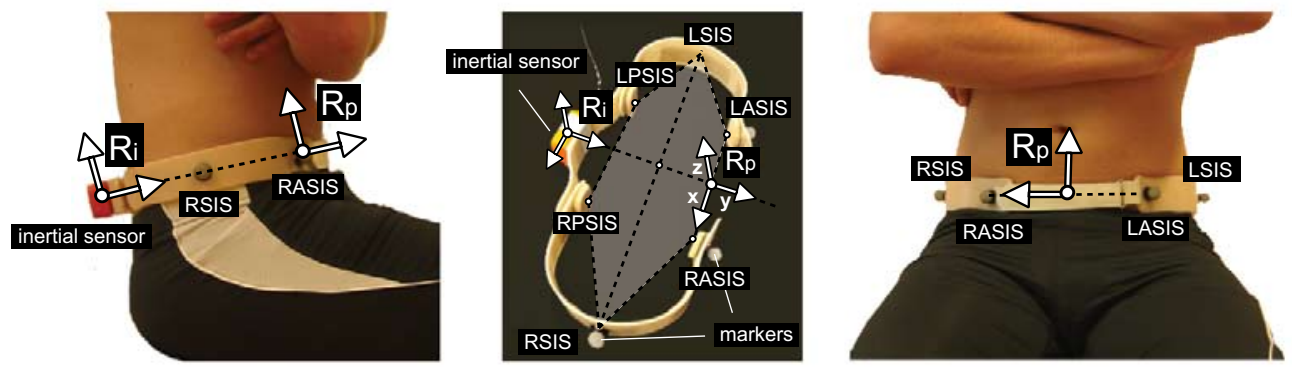

Figure 7.3: Pelvis mold with reflective markers and inertial sensor. The local pelvis frame $\left(\mathrm{R}_{\mathrm{p}}\right)$ is constructed from the reflective markers that were placed on the RASIS, LASIS, RSIS and LSIS. The RPSIS and LPSIS lie in the $\mathrm{x}-\mathrm{y}$ plane. The inertial sensor is attached in a way that the local sensor frame $\left(\mathrm{R}_{\mathrm{i}}\right)$ aligns $\mathrm{R}_{\mathrm{p}}$. The pelvis mold clamped the RASIS, LASIS, RPSIS and LPSIS and was shaped around the left and right lateral iliac crest. $R_{i}$ was used as an alternative measure for $R_{p}$ when the reflective pelvis markers were not visible. 


\subsubsection{Reference Coordinate Frames, Joint Centre Locations and Centre of Masses}

The $\mathrm{x}-\mathrm{z}$ and $\mathrm{y}-\mathrm{z}$ planes of the global reference frame $(\mathrm{G})$ aligned respectively the frontal and sagittal planes of the simulator chair.

The local pelvis frame $\left(\mathrm{R}_{\mathrm{p}}\right)$ was constructed from four markers placed on the PM (Fig 7.3). Two markers were placed on the left and right Anterior Superior Iliac Spines (LASIS and RASIS) and two markers (LSIS and RSIS) on the line between the Anteriorand Posterior Superior Iliac Spines. The x-axis of $\mathrm{R}_{\mathrm{p}}$ ran from the LASIS to the RASIS. The z-axis pointed cranially and was normal to the plane containing the LASIS, RASIS and the midpoint between LSIS and RSIS. The y-axis was the cross product of the $\mathrm{z}$ - and $\mathrm{x}$-axis. The midpoint of RASIS and LASIS defined the origin of $\mathrm{R}_{\mathrm{p}}$. The local coordinates for the lumbosacral joint centre (LJC) and the left and right hip joint centres (LHJC and RHJC) were estimated from pelvic width (distance between RASIS and LASIS) and expressed in $\mathrm{R}_{\mathrm{p}}$ according to the regression equations reported by Dumas et al. (Dumas et al., 2007). In case of problems with pelvis marker visibility, information from the inertial sensor was used to construct a local sensor frame $\left(\mathrm{R}_{\mathrm{i}}\right)$ that we related to $\mathrm{R}_{\mathrm{p}}$ (Fig. 7.3). A local sacrum frame $\left(\mathrm{R}_{\mathrm{s}}\right)$ was constructed from $\mathrm{RHJC}$, LHJC and LJC. The x-axis ran from LHJC to RHJC. The y-axis pointed anterior and was 50 degrees rotated around the $\mathrm{x}$-axis relative to the plane that holds RHJC, LHJC and LJC (Peleg et al., 2007a, Peleg et al., 2007b). The z-axis was the cross product of the $\mathrm{x}$ - and $\mathrm{y}$-axis. LJC defined the origin of $\mathrm{R}_{\mathrm{s}}$. For the left and right thighs, the local coordinate frames $\left(\mathrm{R}_{\mathrm{t}}\right)$ were constructed from the hip joint centres (HJCs), Lateral Femoral Epicondyles (LFEs) and Medial Femoral Epicondyles (MFEs). The knee joint centres (KJCs) were estimated at the midpoint between the LFEs and MFEs. The y-axis of $\mathrm{R}_{\mathrm{t}}$ ran from the HJCs to the KJCs. The z-axis pointed cranially and was normal to the plane containing the HJCs, LFEs and MFEs. The $\mathrm{x}$-axis was the cross product of the $\mathrm{y}$ - and $\mathrm{z}$-axes. HJCs defined the origin of $\mathrm{R}_{\mathrm{t}}$.

The local coordinate frames for the backrest $\left(\mathrm{R}_{1}\right)$, parallelogram $\left(\mathrm{R}_{2}\right)$ and seat support $\left(\mathrm{R}_{3}\right)$ were constructed from angular chair configurations as shown in figure 7.2. The origin of $\mathrm{R}_{3}$ lay on the axes for pelvis adjustment between the ITs.

Body segment masses for the pelvis and thighs were scaled relative to the total body weight and centre of mass positions were estimate relative to $R_{p}$ and $R_{t}$ according to the regression equations as reported by Dumas et al. (Dumas et al., 2007).

\subsubsection{Pelvis Orientation and Low Back Load}

The orientation of $\mathrm{R}_{\mathrm{p}}$ relative to $\mathrm{R}_{3}$ defined pelvis angle in the sagittal and frontal plane by $\alpha_{x}$ and $\alpha_{y}$ respectively. In case of problems with pelvis marker visibility, $R_{i}$ relative to $\mathrm{R}_{3}$ defined pelvis orientation.

The analogue force data was used to calculate the reaction forces on the seat. Nett lumbosacral joint forces $\left(\mathrm{F}_{1}\right)$ and torques $\left(\mathrm{T}_{1}\right)$ were then computed relative to the LJC using static equations of equilibrium for the pelvis and thighs (Fig 7.4). Components of $\mathrm{F}_{1}\left(\mathrm{~F}_{\mathrm{lx}}, \mathrm{F}_{\mathrm{ly}}\right.$ and $\left.\mathrm{F}_{\mathrm{lz}}\right)$ were expressed in $\mathrm{R}_{\mathrm{s}}$ and aligned the $\mathrm{x}-, \mathrm{y}-$, and $\mathrm{z}$-axis respectively. Components of $\mathrm{T}_{1}\left(\mathrm{~T}_{\mathrm{lx}}, \mathrm{T}_{\mathrm{ly}}\right.$ and $\left.\mathrm{T}_{\mathrm{lz}}\right)$ were expressed around the $\mathrm{x}^{-}, \mathrm{y}^{-}$, and $\mathrm{z}$-axis of $\mathrm{G}$. The origin lay in the LJC.

Back interface pressure distribution was derived from the analogue pressure data 

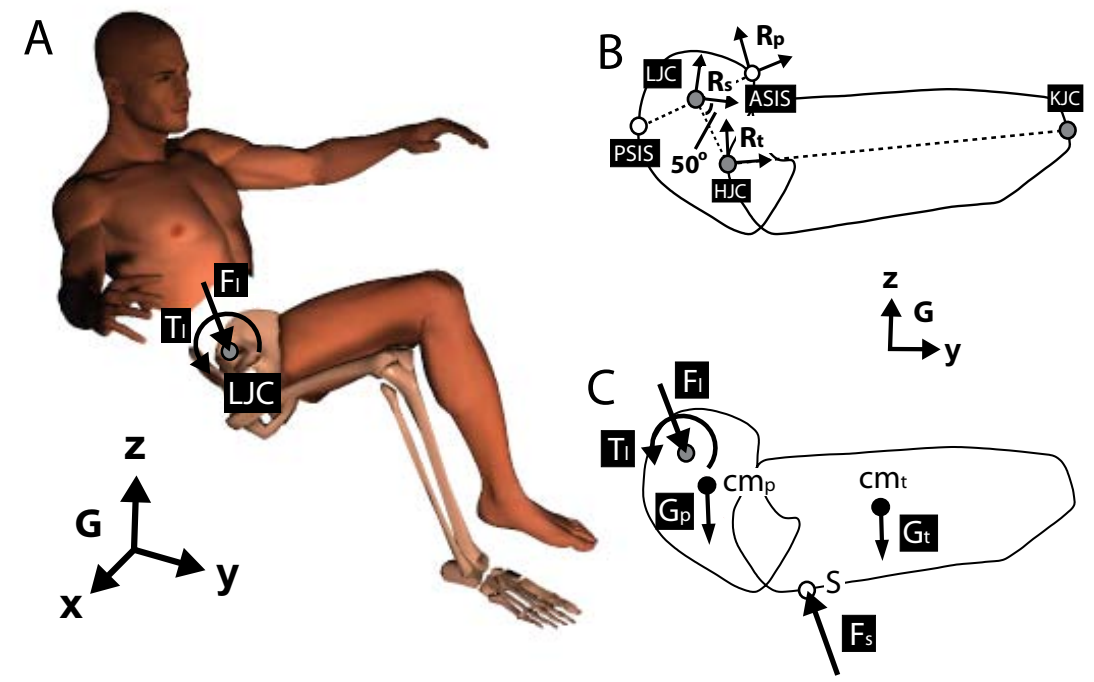

Figure 7.4: The estimation of lumbosacral joint forces and torques in sitting. A: A sitting posture with the net joint forces $\left(\mathrm{F}_{1}\right)$ and torques $\left(\mathrm{T}_{1}\right)$ that exert in the lumbosacral joint centre (LJC). B: The local frames for the sacrum $\left(\mathrm{R}_{\mathrm{s}}\right)$, pelvis $\left(\mathrm{R}_{\mathrm{p}}\right)$ and thighs $\left(\mathrm{R}_{\mathrm{t}}\right)$ are constructed from PSIS, ASIS, LJC, HJC and KJC as shown. C: Individual body segments with forces and torques acting on the thighs and pelvis. The analogue force data was used to calculate the seat reaction force $\left(\mathrm{F}_{\mathrm{s}}\right)$ and its point of application (S). $\mathrm{F}_{1}$ and $\mathrm{T}_{1}$ are computer relative to LJC using static equations of equilibrium for the pelvis and thighs.

as shown in figure 7.5. Low back pressure $\left(\mathrm{P}_{\mathrm{lb}}\right)$ was defined as the average interface pressure as measured in a square contact area $(240 \mathrm{~mm} \times 90 \mathrm{~mm})$ at the level of the lower back. Centre of back pressure (cp) was also derived from the analogue back pressure data and was expressed along the $\mathrm{R}_{1}$ axes.

Prior to the experiment, an empty seated force calibration zero-measurement was performed. Subjects were seated with their ITs on top of the pivot for sagittal pelvis rotation and the anatomical sagittal plane was aligned with the global $y-z$ plane. The footrests were set perpendicular to the seat in, a way that only the weights of the lower legs were supported. The latter was estimated from the gravitational force component on the seat that we monitored during footrest adjustment. The thighs were supported by the seat except for the popliteal space.

For initial angular chair configuration, the seat, parallelogram and backrest were set to $12.5^{\circ}, 37.5^{\circ}$ and $27.5^{\circ}$ respectively. Figure 7.6 shows the protocol for frontal and sagittal pelvis adjustments. In the frontal plane, pelvis rotation was induced from adjusting the relative height between seat parts over a range of $60 \mathrm{~mm}(0 \pm 30 \mathrm{~mm})$. In the sagittal plane, pelvis rotation was induced from adjusting the parallelogram over a range of $35^{\circ}\left(37.5 \pm 17.5^{\circ}\right)$.

Each trial started with a static reference measurement to relate the local inertial sensor frame $\left(\mathrm{R}_{\mathrm{i}}\right)$ to the local pelvis frame $\left(\mathrm{R}_{\mathrm{p}}\right)$. Subjects were then asked to undergo chair movements in a passive way. This meant that they were not allowed to perform any activity that could influence body movement as induced by the chair. 


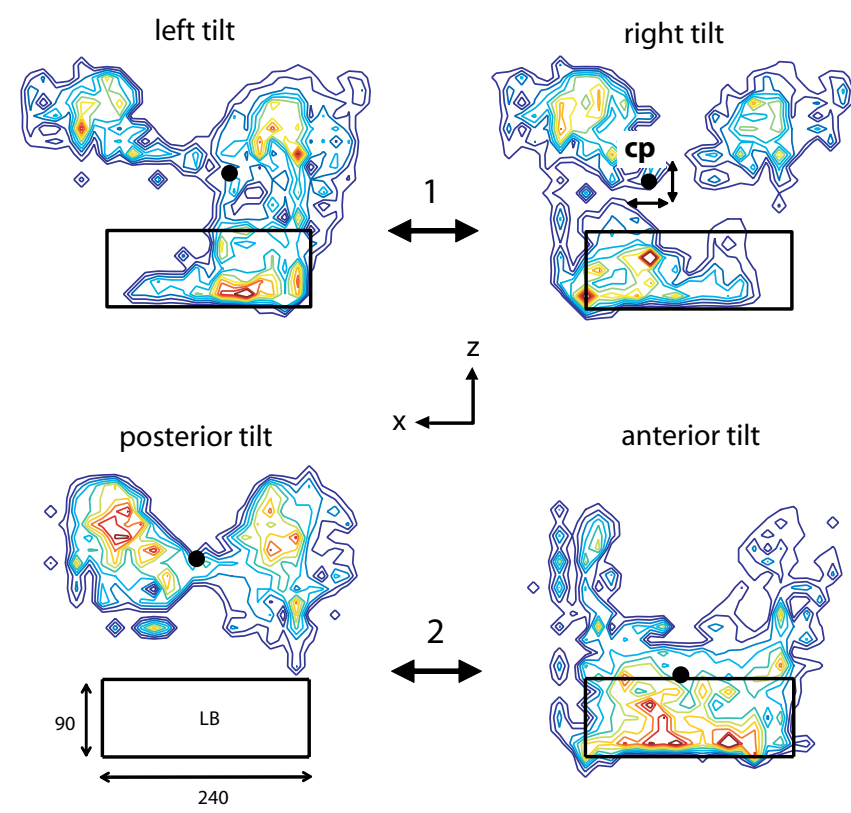

Figure 7.5: Backrest interface pressure distribution during frontal pelvis adjustments (1) and sagittal pelvis adjustment (2). LB represents the square area $(90 \mathrm{~mm} \times 240 \mathrm{~mm})$ in which the average interface pressure was measured for the lower back. Centre of pressure (сp) was expressed along the $\mathrm{x}$ - and $\mathrm{z}$-axis.
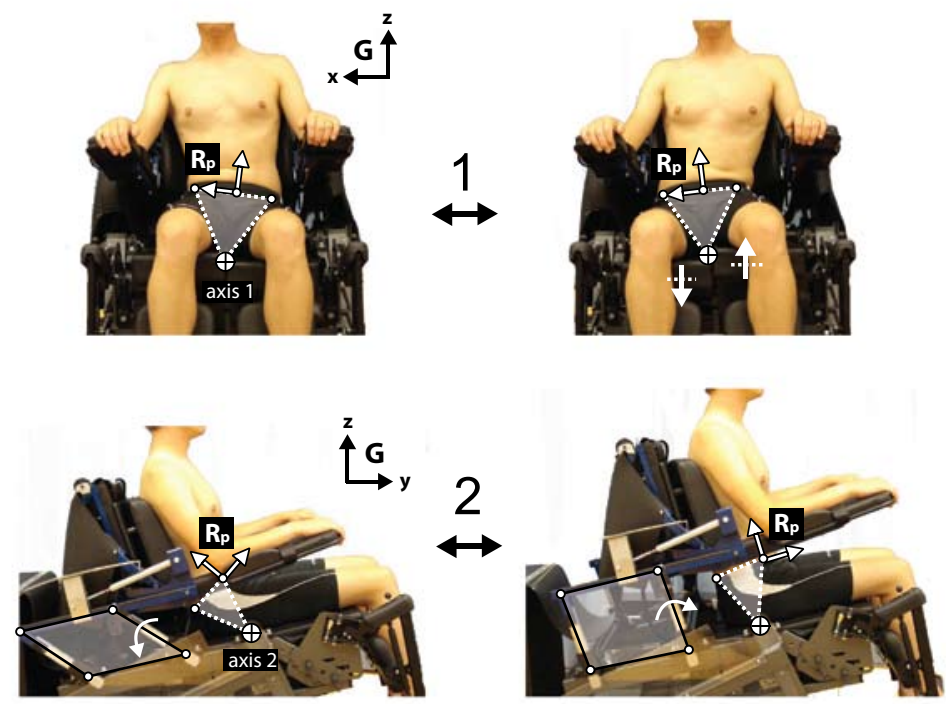

Figure 7.6: Protocol for frontal pelvis adjustment (1) and sagittal pelvis adjustment (2). Pelvis angle is derived from the orientation of the local pelvis frame $\left(\mathrm{R}_{\mathrm{p}}\right)$. $\mathrm{G}$ defines the global reference frame. Adjusting the height between the left and right seat parts rotates the pelvis in the frontal plane around an axis between the ITs (axis 1). Adjusting the configuration of the parallelogram rotates the pelvis in the sagittal plane around an axis under the ITs (axis 2). 
Each trial involved two minutes of quasi-static $(1 / 30 \mathrm{~Hz})$ chair adjustments. The actual measurement only concerned the last 60 seconds.

\subsubsection{Statistics}

For sagittal and frontal pelvis adjustments, the initial values (initial $\pm \mathrm{sd}$ ) and ranges $(\Delta \pm \mathrm{sd})$ for all quantities $\left(\alpha_{\mathrm{x},} \alpha_{\mathrm{y}}, \mathrm{F}_{\mathrm{lx}}, \mathrm{F}_{\mathrm{ly}}, \mathrm{F}_{\mathrm{lz}}, \mathrm{T}_{\mathrm{lx}}, \mathrm{T}_{\mathrm{ly}}, \mathrm{T}_{\mathrm{lz}} \mathrm{cp}_{\mathrm{x}}, \mathrm{cp}_{\mathrm{z}}, \mathrm{P}_{\mathrm{lb}}\right)$ were derived and averaged over all subjects.

To evaluate whether pelvis adjustments were applicable to control low back load, all quantities were first normalized to its total range. Normalized quantities for low back load $\left({ }^{n} F_{l x},{ }^{n} F_{l y},{ }^{n} F_{l z},{ }^{n} T_{l x},{ }^{n} T_{l y},{ }^{n} T_{l z}{ }^{n} \mathrm{Cp}_{x},{ }^{n} \mathrm{Cp}_{z},{ }^{n} P_{l b}\right)$ were then related to normalised pelvis angle $\left({ }^{n} \alpha_{x}\right.$ and $\left.{ }^{n} \alpha_{y}\right)$. For sagittal pelvis adjustment, normalized quantities were related to ${ }^{\mathrm{n}} \alpha_{\mathrm{x}}$, and for frontal pelvis adjustment to ${ }^{\mathrm{n}} \alpha_{\mathrm{y}}$.

Linear relations were assumed and the coefficients of correlation (r) were derived. We then calculated the coefficients of determination $\left(r^{2}\right)$ which gave us an indication about the strength of the relation. Quantities with high coefficients of determination $\left(\mathrm{r}^{2}>0.8\right)$ involved a strong relation with pelvis alignment and were considered controllable from pelvis adjustment.

\subsection{Results}

For sagittal and frontal pelvis adjustments, the initial values (initial $\pm \operatorname{sd})$, ranges $(\Delta \pm$ sd) and coefficients of determination $\left(\mathrm{r}^{2}\right)$ are shown in table 7.2 for all quantities $\left(\alpha_{\mathrm{x}}\right.$ $\left.\alpha_{y}, F_{l x}, F_{l y}, F_{l z}, T_{l x}, T_{l y}, T_{l z} c_{x}, \mathrm{cp}_{z}, P_{l b}\right)$.

Values for $\mathrm{r}^{2}$ were computed from the linear relations between the normalised quantities for low back load $\left({ }^{n} F_{l x},{ }^{n} F_{l y},{ }^{n} F_{l z},{ }^{n} T_{l x},{ }^{n} T_{l y},{ }^{n} T_{l z}{ }^{n} \mathrm{Cp}_{x},{ }^{n} \mathrm{Cp}_{z},{ }^{n} P_{l b}\right)$ and pelvis angle $\left({ }^{\mathrm{n}} \alpha_{\mathrm{x}}\right.$ and $\left.{ }^{\mathrm{n}} \alpha_{\mathrm{y}}\right)$. Linear best-fits together with the coefficients of determination $\left(\mathrm{r}^{2}\right)$ were derived as presented in figure 7.7. This figure shows a selection of the results, which gives us a good indication about the variability between subjects. For frontal pelvis adjustments, the pelvis rotated $9 \pm 1^{\circ}$ and strong relations $\left(r^{2}>0.8\right)$ were found with $\mathrm{cp}_{\mathrm{x}}, \mathrm{T}_{\mathrm{ly}}, \mathrm{T}_{\mathrm{lz}}$. For sagittal pelvis adjustments, the pelvis rotated $19 \pm 3^{\circ}$ and strong relations were found with $\mathrm{cp}_{\mathrm{z}}, \mathrm{F}_{\mathrm{ly}}, \mathrm{F}_{\mathrm{lz}}, \mathrm{T}_{\mathrm{lx}}$ and $\mathrm{P}_{\mathrm{lb}}$.

\subsection{Discussion}

Static sitting in confined settings have been associated with an increased risk for low back pain in sedentary occupations such as office-workers and car drivers. When subjected to prolonged sitting, very little lumbar spine movement is feasible due to the posture imposed configuration of the chair. When performing an active movement task such as reaching forward, lumbar spine movement is constrained between the posterior tilted pelvis and backrest, leaving that largest movement is produced by the thoracic and cervical spine regions (Faiks and Reinecke, 1998). To prevent physical discomfort in prolonged sitting, it is inevitable to design a chair that allows sufficient motion of the lumbar spine. Because the pelvis forms the basis for lumbar spine curvature, we introduced a passive motion technique that adjusts the pelvis relative to the trunk. This study investigated how this technique influences 
Table 7.2: Initial values (initial $\pm \mathrm{sd})$, ranges $(\Delta \pm \mathrm{sd})$ and coefficients of determination $\left(\mathrm{r}^{2}\right)$ for all quantities $\left(\alpha_{\mathrm{x},} \alpha_{\mathrm{y},}, \mathrm{cp}_{\mathrm{x}}, \mathrm{cp}_{\mathrm{z}}, \mathrm{F}_{\mathrm{lx}}, \mathrm{F}_{\mathrm{ly}}, \mathrm{F}_{\mathrm{lz}}, \mathrm{T}_{\mathrm{lx}}, \mathrm{T}_{\mathrm{ly}}, \mathrm{T}_{\mathrm{lz}}, \mathrm{P}_{\mathrm{lb}}\right)$ in frontal and sagittal pelvis adjustments.

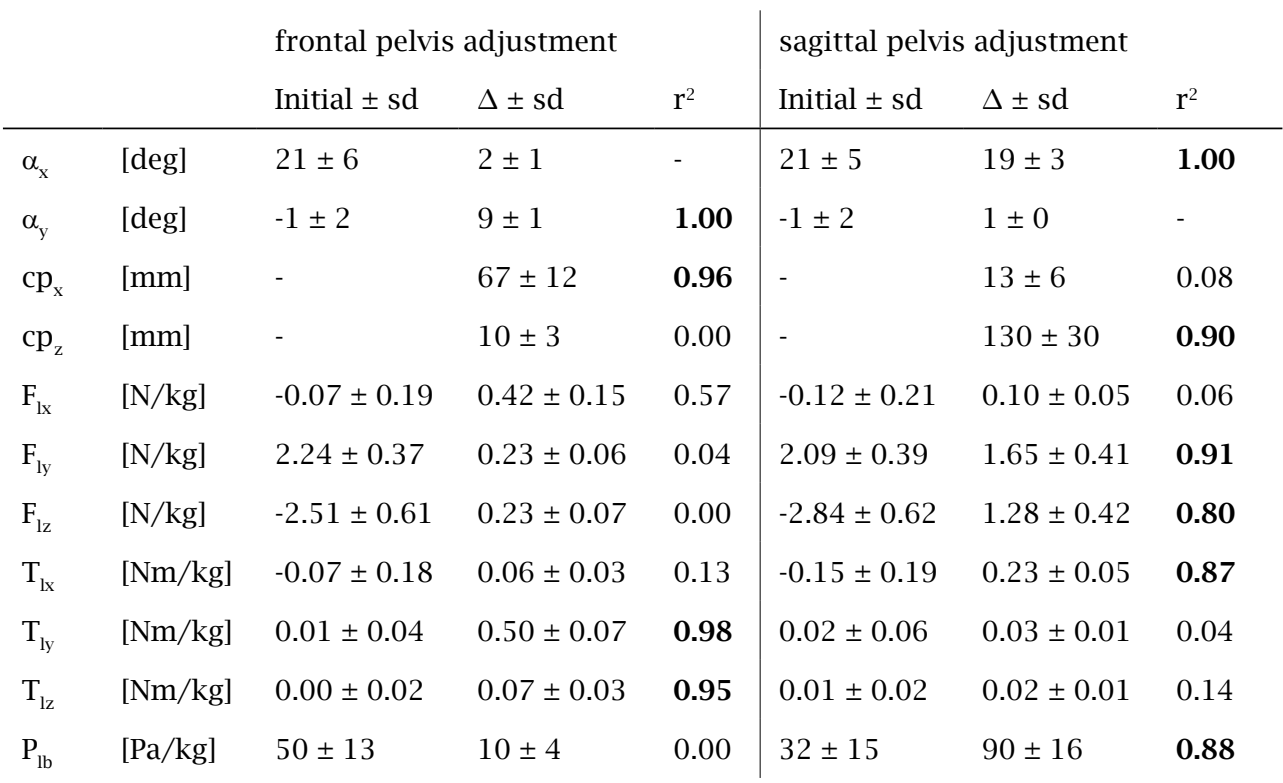

Bold values for $\mathrm{r}^{2}$ involve strong correlations $\left(\mathrm{r}^{2}>0.8\right)$ with pelvis rotation.

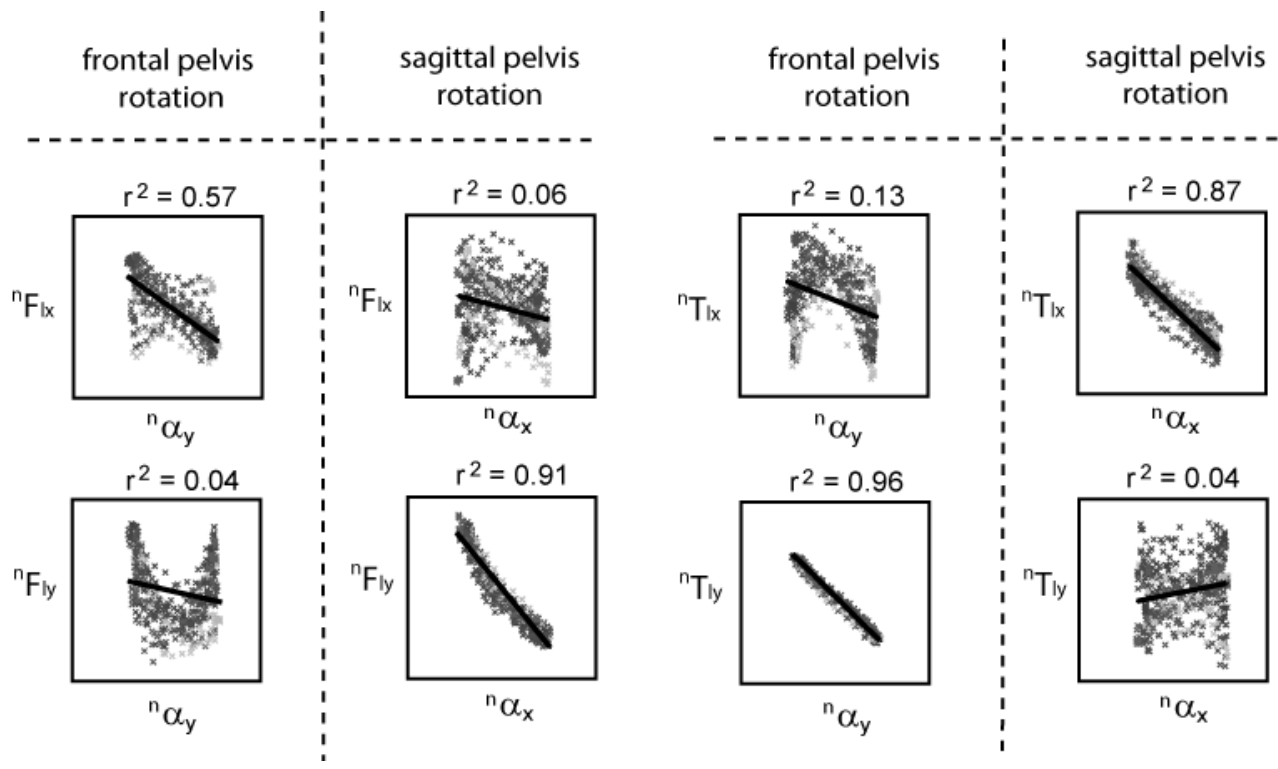

Figure 7.7: A selection of the results in which some normalised quantities for low back load $\left({ }^{\mathrm{n}} \mathrm{F}_{\mathrm{lx}}\right.$, ${ }^{n} F_{l y},{ }^{n} T_{l x}$ and $\left.{ }^{n} T_{l y}\right)$ are related to pelvis angle $\left({ }^{n} \alpha_{x}\right.$ and $\left.{ }^{n} \alpha_{y}\right)$ for all subjects. Linear best-fits together with the coefficients of determination $\left(\mathrm{r}^{2}\right)$ are shown, which gives us a good indication about the variability between subjects. 
the loads at the lower back, and to what extend low back load can be controlled from passive pelvis adjustments.

Our findings suggest that decoupled pelvis adjustments are effective to rotate the pelvis relative to the trunk and to control low back load in healthy individuals. In frontal and sagittal direction, the pelvis rotated up to $9^{\circ}$ and $19^{\circ}$ respectively. Significant relations $\left(\mathrm{r}^{2}>0.8\right)$ were found between pelvis rotation and almost all quantities of low back load which we considered controllable from decoupled pelvis adjustments.

During pelvis movement we observed minimal variations in trunk orientation. This means that the imposed pelvis rotation mainly contributed to deformation of the lumbar spine. As an indication for the mechanical conditions of the lower back, we evaluated the net lumbosacral joint forces $\left(\mathrm{F}_{1}\right)$ and torques $\left(\mathrm{T}_{1}\right)$, and the external lower back pressure $\left(\mathrm{P}_{\mathrm{lb}}\right)$. Centre of back pressure $(\mathrm{cp})$ was computed to observe how back pressure redistributed during continuous pelvis rotation. We associate $\mathrm{T}_{1}$ and $\mathrm{F}_{\mathrm{lz}}$ with intervertebral disc compression, and $\mathrm{F}_{\mathrm{lx}}$ and $\mathrm{F}_{\mathrm{ly}}$ with the amount of shear in lateral and forward direction respectively.

Sagittal pelvis adjustments seemed effective to control the interface pressure at the lower back $\left(\mathrm{P}_{\mathrm{lb}}\right)$, centre of back pressure in upward direction $\left(\mathrm{cp}_{\mathrm{z}}\right)$, and the lumbosacral joint forces and torque in the sagittal plane $\left(\mathrm{F}_{\mathrm{ly}}, \mathrm{F}_{\mathrm{lz}}\right.$ and $\left.\mathrm{T}_{\mathrm{lx}}\right)$. The significant effects that we observed on $\mathrm{T}_{\mathrm{lx}}$ and $\mathrm{F}_{\mathrm{lz}}$, suggests that sagittal pelvis adjustments might be effective to control pressures at the intervertebral discs.

When adjusting the pelvis rearward, lumbar spine posture changes from a lordotic to a kyphotic curvature. This makes that the thoracic region takes up more body load which corresponded to the decrease in $\mathrm{P}_{\mathrm{lb}}$ and upward shift of $\mathrm{cp}$. The opposite effect is shown when adjusting the pelvis forward. More body load exerts on the lower back and cp travels downward. Similar effects have been reported by Makhsous et al. (Makhsous et al., 2003) who evaluated the effects of an adjustable ischial support. When adjusting the ischial support downward, they found that the pelvis rotated forward and more load exerted on the backrest. They also reported an enhanced total and segmental lumbar lordosis, and an increase in lumbar intervertebral disc height, which they associated with reduced low back pain.

In the frontal plane we found significant effects of $\alpha_{\mathrm{y}}$ on $\mathrm{T}_{\mathrm{ly}}$ and $\mathrm{T}_{\mathrm{lz}}$. Especially the latter might be interesting concerning the mechanical spine conditions, because this indicates that frontal pelvis motion could influence spinal axial-torsion as well. Van Deursen et al. (van Deursen et al., 1999) suggested in a previous study that manipulation of the spinal tissue around the axial direction might reduce lumbar discomfort in prolonged static sitting. With this in mind they designed an ergonomic office chair that potentially induced axial spinal torsion from extremely small axial seat rotations. However, after two years of clinical observation in 280 office-workers suffering from LBP, Lengsfeld et al. (Lengsfeld et al., 2007) concluded that passive rotary dynamic sitting was not superior to sitting in other high-quality office chairs. They argued that the implemented seat rotation did not induce enough variation in spinal load and suggested that considerable higher rotation amplitudes might provide better outcomes with regard to lumbar pain relief. How this relates to socalled decoupled pelvis rotation in frontal direction is interesting to evaluate.

It is very hard to interpret our findings in terms of the potential risk for lumbar discomfort since we did not measure the mechanical and physiological lumbar spine 
conditions at inter-segmental level. However, we do think that these findings concern interesting information about the response of the lower lumbar spine to continuous pelvis rotation.

Besides a potential benefit for car drivers and office-workers, the described seating intervention might also be applicable for wheelchair users who suffer from trunk instability and lack functional movement during daily wheelchair use. To prevent mobility problems from continuous static sitting, wheelchair users who cannot functionally reposition themselves might benefit from periodic decoupled pelvis adjustments as well. Because the induced pelvis motion does not affect the orientation of the upper body, minimal drawback is expected on most activities of daily life.

This study demonstrated that the described technique for decoupled pelvis adjustment seems effective to control low back load from induced lumbar motion. However, the unavoidable questions arise how long certain stress levels can be sustained and how often the pelvis must be adjusted to prevent that tissue degeneration takes place. The US Department of Health recommends individuals to shift body weight every 15 minutes (Agency for Health Care Policy and Research, 1994). In a recent study, Linder-Ganz et al. (Linder-Ganz et al., 2007) evaluated healthy sitting behaviour by quantifying the amount and frequency of postural change during prolonged sitting. They reported that the frequency of postural change found in healthy subjects was higher than recommended by the Agency for Health Care Policy. Linder-Ganz et al. found that healthy individuals moved approximately every 6 minutes in the sagittal plane and every 9 minutes in the frontal plane. Continuous pelvis adjustment in both directions and with various frequencies and extent might tell us which protocol of induced lumbar motion benefits persons with low back pain the most. The implementation of decoupled pelvis adjustment as a passive motion technique in car seats or new generation office-chair in an interesting next step. Future research must aim at the beneficial effects on lumbar pain relief and demonstrate the influence on task performance in people who do sedentary work and sit still in confined stetting for hours.

\subsection{Conclusions}

We conclude that decoupled pelvis adjustments might be an applicable passive motion technique to control low back load in sitting. Because the current study only focused on the net lumbosacral joint forces and the pressures at the backrest interface, future studies must address the mechanical and physiological lumbar spine conditions on inter-segmental level. This might provide a better foundation for a potential benefit on lumbar pain relief in prolonged static sitting.

\subsection{Acknowledgements}

This study was partly funded by the Dutch Ministry of Economical Affairs, SenterNovem. The authors would like to thank the engineering company Demcon (Oldenzaal, the Netherlands) for developing the experimental simulator chair. The authors also thank Birgit Molier and Karel van den Hengel for their assistance during the experiments. 


\section{Part IV}

\section{Clinical Evaluation}




\section{Chapter 8}

\section{Seating Interventions that Regulate Body Load in Spinal Cord Injured Individuals}

To be submitted as: P. van Geffen, J. Reenalda, G.J. Snoek, P.H. Veltink and H.F.J.M. Koopman. A combination of two seating interventions to regulate body load in spinal cord injured individuals. 


\begin{abstract}
For wheelchair-users who cannot physically reposition themselves and suffer from pressure ulcers and low back pain due to prolonged static sitting, dynamic seating interventions are needed that periodically alleviate the load bearing tissue under the buttock and at the lower back.

A computer-aided adjustable simulator chair was developed to study the influence of decoupled pelvis rotation (DPR) and tuberal support adjustment (TSA) on pelvis alignment, subtuberal blood supply, centre of pressure, and the forces that acted on the tuberal supports and lumbosacral spine in ten male subjects with a spinal cord injury (SCI).

$D P R$ rotated the pelvis $10.9 \pm 2.6$ degrees and significantly $(p<0.05)$ influenced centre of pressure and the net forces that acted in the lumbosacral spine. In TSA, significant effects were found on the supply of blood to the subtuberal buttock tissue and the contact forces that exerted on the tuberal supports.

A combination of DPR and TSA might therefore be useful for simultaneous regulation of body load to prevent pressure ulcers and low back pain in SCI-individuals who lack the strength to reposition themselves physically.
\end{abstract}




\subsection{Introduction}

Impairments to the neuromuscular function make many wheelchair-users suffer from reduced trunk stability (Seelen et al., 2001). To maintain balance during activities of daily life, a 'slouched' static body posture is often adopted (Hobson and Tooms, 1992). Although the body support forces seem reasonable in sitting, sustained mechanical tissue loading will damage body structures and causes all kinds of physical discomfort.

\subsubsection{Physical discomfort from prolonged static sitting}

It has been reported that the mechanical lumbar spine characteristics change in prolonged sitting with flexed lumbar spine curvature (Argoubi and Shirazi-Adl, 1996, Hedman and Fernie, 1997, Beach et al., 2005, Parkinson et al., 2004, Keller et al., 1987). Sustained mechanical spinal loading without significant durations of stress relief causes nutrient supply to stagnate and load bearing spinal structures to degenerate (Urban et al., 2004). Because the intervertebral discs and facet joints are important structures to maintain segmental stability, degenerative processes in these structures change the segment motion characteristics associated with spinal instability and low back injury (Fujiwara et al., 2000a, Fujiwara et al., 2000b). A lordotic lumbar spine curvature together with significant levels of spinal movement are needed to facilitate the perfusion of intervertebral disc nutrition (Holm and Nachemson, 1983) and to decrease the risk for disc degeneration (Urban et al., 2004).

Static sitting has also been associated with pressure ulcers which are characterized places of tissue breakdown in skin or underlying soft tissues (Bouten et al., 2003). Forces that exert on the body surface are transferred into underlying tissue and cause body structures to deform. Continuous stressing the buttock tissue potentially initiates the irreversible physiological process of tissue necrosis (Swain and Bader, 2002, Bouten et al., 2003). Successive durations of pressure relief are needed to recover the buttock tissue from deformation and blood flow stagnation.

\subsubsection{Dynamic seating interventions}

Most mobility problems occur from sustained mechanical tissue loading and dynamic seating interventions are therefore needed to periodically relieve body structures associated with wheelchair seating discomfort (Crane et al., 2007).

To reduce incidence of low back injury in static sitting, several techniques have been reported to enhance lumbar motion and relieve static loads that cause spinal tissue degeneration and pain (van Deursen et al., 1999, Aota et al., 2007, Reinecke et al., 1994). Van Deursen et al. (van Deursen et al., 1999) described an ergonomic chair that uses passive motion for continuous axial pelvis rotation imposed by small rotational seat movements in the horizontal plane. Based on subjective measures for lumbar discomfort, they reported a positive effect on pain relief in subjects suffering low back injury. Reinecke et al. (Reinecke et al., 1994) described a commercially available device (Backcycler, ergonomics Inc., Winooski, VT) that induces passive sagittal motion to the lower spine by an alternating inflatable lumbar support. They reported an improved tolerance for prolonged sitting when subjected to continuous lumbar 
motion. Atoa et al. (Aota et al., 2007) also investigated the effects of sagittal lumbar adjustments in prolonged static sitting. Although no clear evidence was found that this technique contributes to a reduced feeling of lumbar discomfort, they did report significant improvement for buttock numbness. This latter was explained from pressure changes at the buttock-seat interface because lumbar adjustment did affect pelvis alignment as well.

Besides its potential benefit for lumbar pain relief, a passive motion technique that adjusts pelvis angle relative to the trunk and seat support might therefore also be applicable to redistribute buttock load and reduce the risk for pressure ulcers in prolonged wheelchair sitting (Stockton and Rithalia, 2007). When static seating surfaces do not relieve buttock pressure enough, dynamic systems that adjusts the seating surface are useful within proper pressure management (Makhsous et al., 2007, Stockton and Rithalia, 2007). However, it must never be the intention to apply dynamic seating surfaces and completely replace the need for postural movement. A combination of both might therefore be most effective, especially when static sitting is also associated with mobility problems other than pressure ulcers.

With this in mind, we developed a computer-aided simulator chair equipped with adjustable support elements under the ischial tuberosities (ITs) and an intervention that rotates the pelvis independent from the backrest and seat support. Although the effects on pelvis alignment (van Geffen et al., 2008a), low back load [chapter 7], buttock pressure distribution (van Geffen et al., 2008b) and subtuberal blood supply [chapter 6] have already been demonstrated in able-bodied, no clinical investigation has yet been performed on impaired subjects as well.

\subsubsection{Objectives and hypotheses}

This paper studies the effects of so-called decoupled pelvis rotation (DPR) and tuberal support adjustment (TSA) on pelvis alignment and body load in subjects with a spinal cord injury (SCI). Because the pelvis is supported by the seat and directly affects the curvature of the lower spine, we expect large influence of DPR on centre of pressure and the forces that act at the lower back. Based on our previous observation that the pelvis roughly rotates around an axis under the ITs, we do not expect large effects on the forces in this region. To influence the forces under the ITs more directly, TSA might be a good alternative. Because we assume that this does not influence pelvis alignment, we expect no additional effects on the rest of the buttock-seat interface and lower back. The main objective of this study is twofold. We investigate the contribution of DPR and TSA on pelvis alignment, subtuberal blood supply, centre of pressure, and the forces that act under the ITs and lower back in spinal SCI-individuals and we evaluate how these quantities can be regulated in prolonged static sitting.

\subsection{Methods}

\subsubsection{Subjects}

Ten male subjects (age $57.6 \pm 7.4$ years, weight $87.3 \pm 14.1 \mathrm{~kg}$, length $1.80 \pm 0.07$ $\mathrm{m})$ with a spinal cord injury (SCI) were recruited for this study. Before and after the 
experiment, an experienced nurse carefully checked the ischial buttock region to be sure that there was no skin damage or other signs of pressure sores. In all subjects, no such problems were observed. Furthermore, the study was approved by the local Committee for Medical Ethics and all subjects read and signed an 'informed consent' in which the objective and experimental protocol was explained.

\subsubsection{Instrumentation}

Experiments were performed with a computer-aided adjustable simulator chair instrumented with a parallelogram design that allows sagittal pelvis rotation without adjusting the orientations of the backrest and seat support (fig. 8.1). This concept for so-called decoupled pelvis rotation (DPR) is discussed in a previous study which demonstrated the effects on sagittal pelvis alignment in healthy male subjects (van Geffen et al., 2008a). The principle is based on a parallelogram that aligns the pivots for chair modification with the anatomical axes for pelvis and trunk rotation.

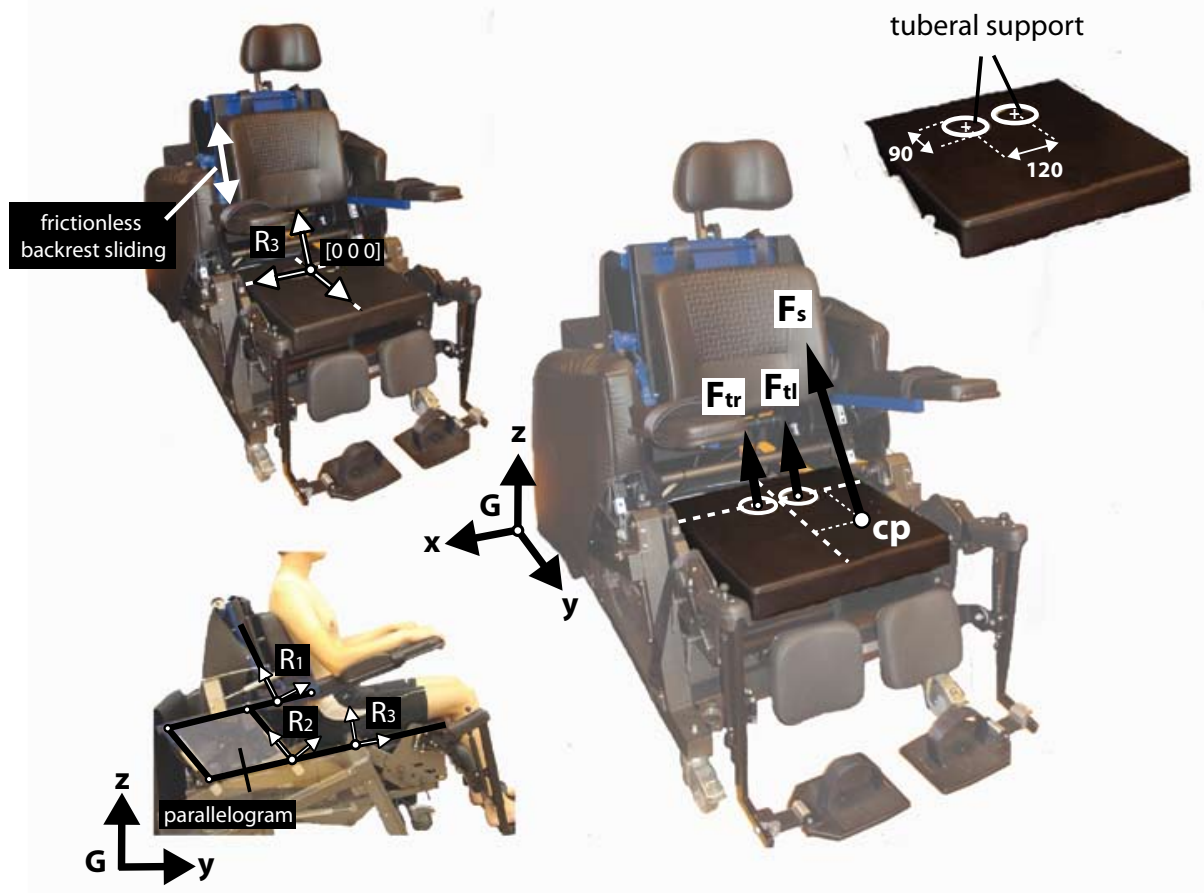

Figure 8.1: Computer-aided adjustable simulator chair with parallelogram design for decoupled pelvis rotation (DPR). Spaced according to an inter-tuberal distance of $120 \mathrm{~mm}$, two adjustable tuberal supports $(\varnothing 90 \mathrm{~mm})$ are integrated in the seating surface. Six uni-axial load cells are mounted under the seat support to measure centre of pressure $(\mathrm{cp})$ and seat reaction force $\left(\mathrm{F}_{\mathrm{s}}\right)$. Two more load cells are mounted under the tuberal supports to measure the normal forces under the tuberosities $\left(\mathrm{F}_{\mathrm{t}}=\mathrm{F}_{\mathrm{tr}}+\mathrm{F}_{\mathrm{tl}}\right)$. The backrest is mounted on a sledge. Because the weight of the backrest is counterbalanced, frictionless vertical trunk movements are allowed. G defines the global reference frame. The local coordinate frames for the backrest $\left(\mathrm{R}_{1}\right)$, parallelogram $\left(\mathrm{R}_{2}\right)$ and seat support $\left(\mathrm{R}_{3}\right)$ were constructed from angular chair configurations. $\mathrm{F}_{\mathrm{s}}$ and $\mathrm{cp}$ are expressed in $R_{3}$ relative to the origin $\left[\begin{array}{lll}0 & 0 & 0\end{array}\right]$. 
Adjustment of the parallelogram configuration rotates the pelvis in sagittal direction around an axis under the ITs. The backrest is mounted on a sledge to allow vertical trunk translations and to align the axes for decoupled pelvis rotation exactly with the axes for chair adjustment. The weight of the backrest is counterbalanced and the seat support, parallelogram and footrests are adjustable to align body posture with chair configuration.

The simulator chair is also instrumented with an adjustable force sensing seating plane to manipulate and monitor ischial buttock load simultaneously. On top of the axis for sagittal pelvis rotation and spaced according to an average inter-tuberal distance of $120 \mathrm{~mm}$, two adjustable force sensing support elements (Ø 90mm) are integrated in the seating surface. To relieve subtuberal buttock load periodically, both support elements are downward adjustable within a range of 40mm.

For accurate measurement of seat reaction forces, six more uni-axial load cells $\left(\right.$ FUTEK $^{\circledR}$, California, USA) are mounted under the seat support. Reflective markers were placed on selected anatomical landmarks. Three-dimensional body segments orientation was obtained with an infrared camera motion capturing system (VICON ${ }^{\circledR}$, Oxford, UK) from reflective markers that were placed on the trunk, pelvis and thighs.

To prevent skin artefacts during pelvis movement, a pelvis mold (PM) was shaped around the left and right lateral iliac crest and clamped the anterior- and posterior superior iliac spines for optimal fixation. To prevent problems with pelvis marker visibility, an inertial sensor (MT ${ }^{\circledR}$, Xsens, Enschede, the Netherlands) was attached on the PM as an alternative to estimate pelvis orientation. The line of gravity was used to derive sensor inclination that we related to the orientation of the PM as shown in figure 8.2.

Subcutaneous subtuberal buttock tissue oxygenation and perfusion were simultaneously measured with a non-invasive fibre-optic probe that we attached on the skin under the right ischial tuberosity. The probe was connected with the O2C (Oxygen-to-see ${ }^{\circledR}$, LEA, Giessen, Germany) which is a diagnostic device that uses light-guide tissue spectrophotometry for simultaneous non-invasive measurement of oxygen supply in blood perfused tissue up to a depth of approximately $8 \mathrm{~mm}$. The probe incorporates Laser-Doppler and broadband light spectroscopy and was shaped with relative flat dimensions of $12 \times 5.5 \times 45.5 \mathrm{~mm}$ ( $\mathrm{W} \times \mathrm{H} \times \mathrm{L}$ ). It was designed to withstand high contact force so that it could measure sub-tuberal blood supply in fully loaded sitting conditions.

The level of subcutaneous tissue perfusion is called $\mathrm{BF}_{\mathrm{sc}}$ and reflects the blood flow in the microcirculation as an arbitrary unit [au]. The level of subcutaneous tissue oxygenation is called $\mathrm{SO} 2_{\mathrm{sc}}$ and reflects oxygen saturation of hemoglobin as a relative measure [\%].

\subsubsection{Data Analysis}

The $x-z$ and $y-z$ planes of the global reference frame (G) are aligned with respectively the frontal and sagittal planes of the simulator chair. The local pelvis frame $\left(\mathrm{R}_{\mathrm{p}}\right)$ was constructed from four markers on the PM (fig 8.2). Two markers were placed on the left and right anterior superior iliac spines (LASIS and RASIS) and two markers (LSIS and RSIS) on the line between the anterior- and posterior superior iliac spines. 


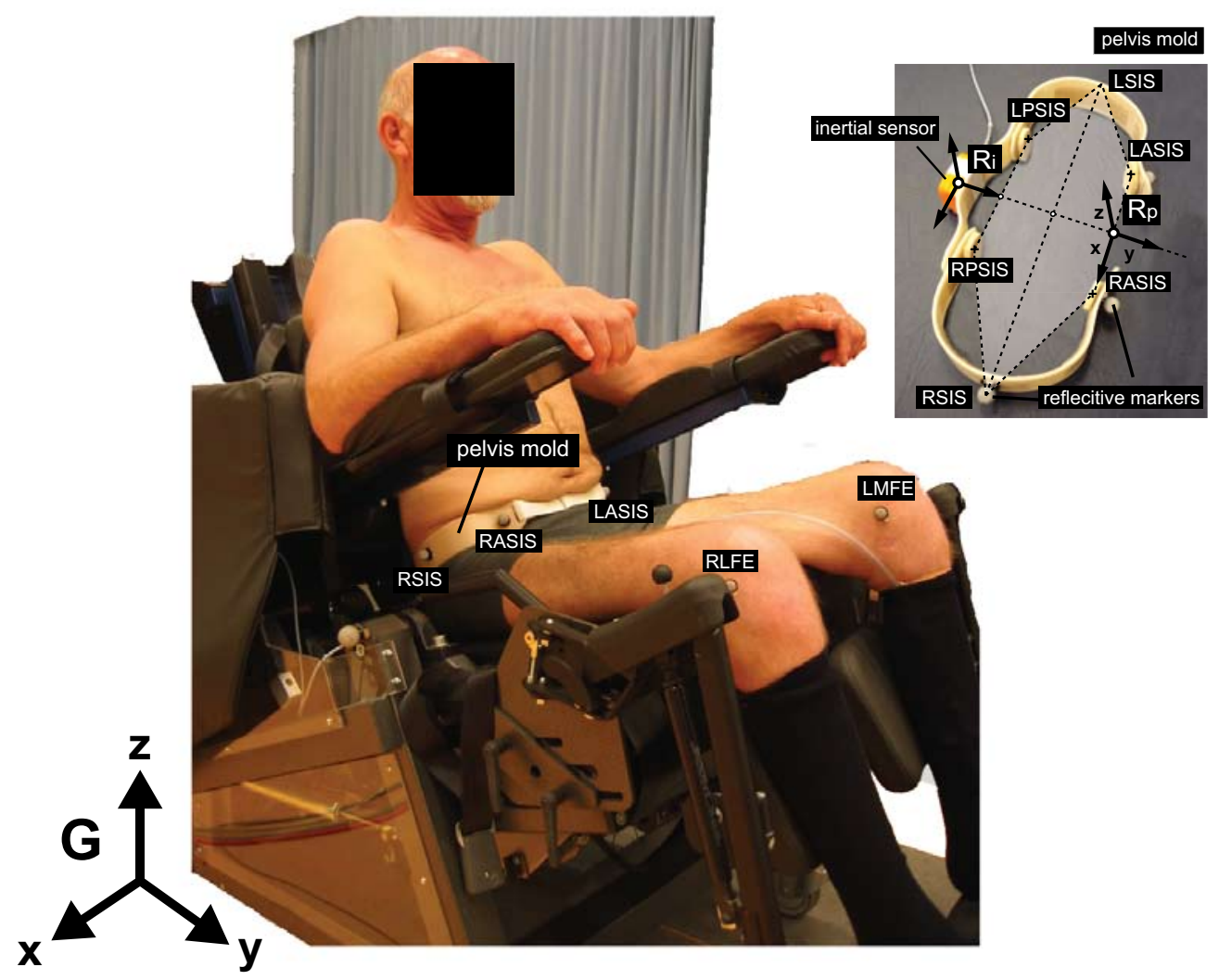

Figure 8.2: Subjects were seated with their ischial tuberosities top of the tuberal supports. The subject's anatomical sagittal plane was carefully aligned with the $y$-z plane of G. A pelvis mold with reflective markers and inertial sensor was shaped around the lateral iliac crests and clamped the anterior- and posterior superior iliac spines for fixation. The local pelvis frame $\left(\mathrm{R}_{\mathrm{p}}\right)$ is constructed from the reflective markers that were placed on the RASIS, LASIS, RSIS and LSIS. The RPSIS and LPSIS lie in the $x-y$ plane. The inertial sensor is attached in a way that the local sensor frame $\left(\mathrm{R}_{\mathrm{i}}\right)$ aligns $\mathrm{R}_{\mathrm{p}}$.

The x-axis of $\mathrm{R}_{\mathrm{p}}$ runs from the LASIS to the RASIS. The $\mathrm{z}$-axis points cranially and is normal to the plane containing the LASIS, RASIS and the midpoint between LSIS and RSIS. The y-axis is the cross product of the z- and x-axis. The midpoint of RASIS and LASIS defined the origin of $\mathrm{R}_{\mathrm{p}}$.

Furthermore, three reflective markers were placed on the trunk including the $7^{\text {th }}$ cervical (C7), upper sternum (US) and processus xypoid (PX). The local coordinates for the lumbosacral joint centre (LJC) and the left and right hip joint centres (LHJC and RHJC) were expressed in $\mathrm{R}_{\mathrm{p}}$ and estimated from pelvic width according to the regression equations as reported by Dumas et al. (Dumas et al., 2007). In case of problems with pelvis marker visibility, information from the inertial sensor was used to construct a local sensor frame $\left(\mathrm{R}_{\mathrm{i}}\right)$ that we related to $\mathrm{R}_{\mathrm{p}}$. A local sacrum frame $\left(\mathrm{R}_{\mathrm{s}}\right)$ was constructed from RHJC, LHJC and LJC. The x-axis runs from LHJC to RHJC. The $\mathrm{y}$-axis points anterior and is 50 degrees rotated around the $\mathrm{x}$-axis relative to 
the plane that holds RHJC, LHJC and LJC (Peleg et al., 2007). The z-axis is the cross product of the $\mathrm{x}$ - and $\mathrm{y}$-axis. LJC defined the origin of $\mathrm{R}_{\mathrm{s}}$. The left thigh frame $\left(\mathrm{R}_{\mathrm{tl}}\right)$ is constructed from LHJC, and two reflective markers that were placed on the left lateral femoral epicondyle (LLFE) and left medial femoral epicondyle (LMFE). The left knee joint centre (LKJC) is estimated at the midpoint between the LLFE and LMFE. The $\mathrm{y}$-axis of $\mathrm{R}_{\mathrm{tl}}$ runs from the LHJC to the LKJC. The z-axis points cranially and is normal to the plane containing the LHJC, LLFE and LMFE. The $\mathrm{x}$-axis is the cross product of the $y$ - and $z$-axes. LHJC defined the origin of $\mathrm{R}_{\mathrm{tl}}$. The right thigh frame $\left(\mathrm{R}_{\mathrm{tr}}\right)$ is constructed from RHJC, and two markers that were placed on the right lateral femoral epicondyle (RLFE) and right medial femoral epicondyle (RMFE). The right knee joint centre (RKJC) is estimated at the midpoint between the RLFE and RMFE. The $y$-axis of $R_{t r}$ runs from the RHJC to the RKJC. The z-axis points cranially and is normal to the plane containing RHJC, RLFE and RMFE. The $\mathrm{x}$-axis is the cross product of the $y$ - and $z$-axes. RHJC defined the origin of $R_{t r}$. The local coordinate frames for the backrest $\left(R_{1}\right)$, parallelogram $\left(R_{2}\right)$ and seat support $\left(R_{3}\right)$ were constructed from angular chair configurations as shown in figure 8.1. The origin of $\mathrm{R}_{3}$ lies in the middle of the seat support, on top of the axes for sagittal pelvis rotation and in-between the adjustable tuberal support elements.

Body segment masses for the pelvis and thighs were scaled relative to the total body weight and centre of mass positions were estimate relative to $R_{p}, R_{t l}$ and $R_{t r}$ according to the regression equations as reported by Dumas et al. (Dumas et al., 2007). Pelvis angle $(\alpha)$ was expressed relative to the seat support and computed from the helical angle between $\mathrm{R}_{\mathrm{p}}$ and $\mathrm{R}_{3}$ in the sagittal plane. In case of problems with pelvis marker visibility, $\alpha$ was computed from the sagittal angle between $R_{i}$ and $R_{3}$. The seat resultant force $\left(\mathrm{F}_{\mathrm{s}}\right)$ was derived from the force sensors under the seat support. Centre of pressure $(\mathrm{cp})$ is expressed along the $y$-axis of $R_{3}$. The tuberal normal force $\left(F_{t}\right)$ was obtained from both force sensing tuberal support elements and expressed along the $\mathrm{Z}$-axis of $\mathrm{R}_{3}$. Typical representations of reflective body markers, seat resultant force and centre of pressure in DPR and TSA are shown in fig. 8.3.
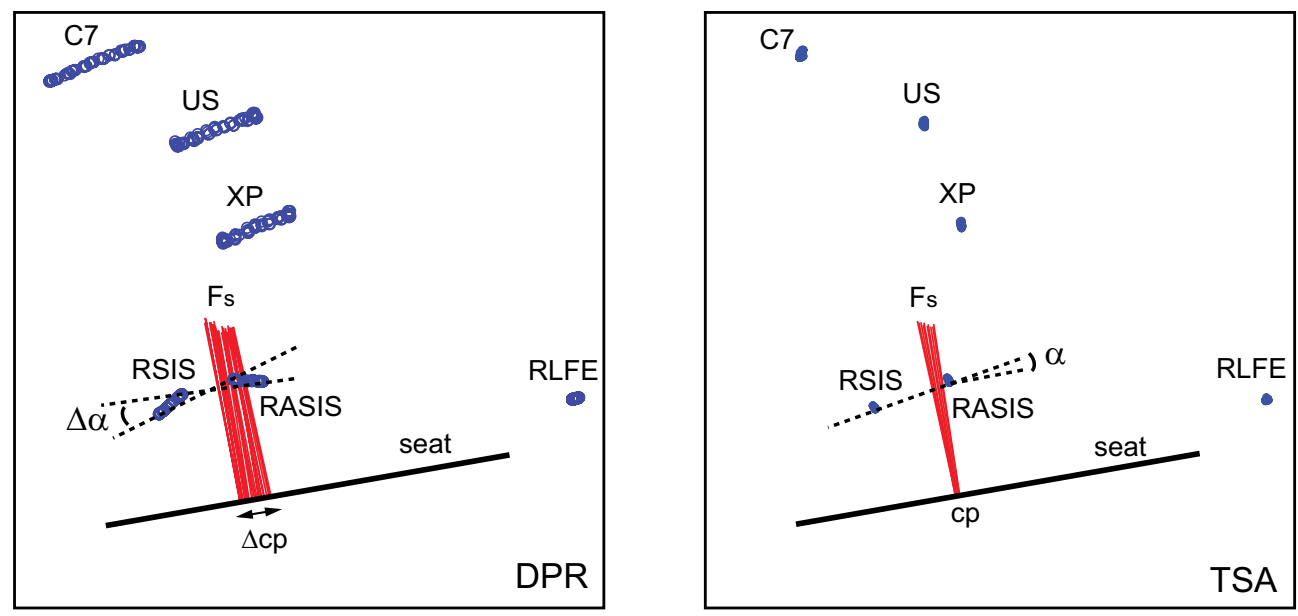

Figure 8.3: Typical representation of reflective body markers (C7, US, XP RSIS, RASIS and RLFE), seat resultant force $\left(\mathrm{F}_{\mathrm{s}}\right)$ and centre of pressure $(\mathrm{cp})$ in DPR and TSA. 
The net lumbosacral joint forces $\left(\mathrm{F}_{1}\right)$ and torques $\left(\mathrm{T}_{1}\right)$ were computed relative to the LJC using static equations of equilibrium for the pelvis and thighs (fig. 8.4). Components of $F_{1}\left(F_{l y}\right.$ and $\left.F_{l z}\right)$ are expressed in $R_{s}$ and align the $y^{-}$, and $z$-axis respectively. $T_{1}$ was expressed around the $\mathrm{x}$-axis of $\mathrm{R}_{\mathrm{s}}$.

\subsubsection{Experimental protocol}

Prior to the experiment, an empty seated zero-force calibration measurement was performed. Subjects were first transferred with a transfer lift from their wheelchair to a bed on which they lied down with their legs pulled up and their hip and knee joints flexed 90 degrees. In this posture, the ITs were palpated by an experienced nurse who carefully attached the O2C probe on the skin below the right ITs. Subjects were then transferred with the transfer lift to the experimental simulator chair. They were seated with the ITs top of the tuberal support elements and the anatomical sagittal plane aligned with the global $y$-z plane. The thighs were fully supported by the seat except for the popliteal space. The footrests were set perpendicular to the seat so that they only supported the weights of the lower legs and no joint forces acted in the knees. This latter was estimated from the gravitational force component on the seat that we monitored during footrest adjustment.

Reflective markers were placed and initial angles for the seat support and backrest were set respectively to $10^{\circ}$ and $110^{\circ}$ relative to the horizontal. The experiment involves two trials. During the first trial, periodic quasi-static $(1 / 30 \mathrm{~Hz})$ decoupled pelvis rotation (DPR) was imposed from sinusoid parallelogram angle $(\beta)$ adjustment

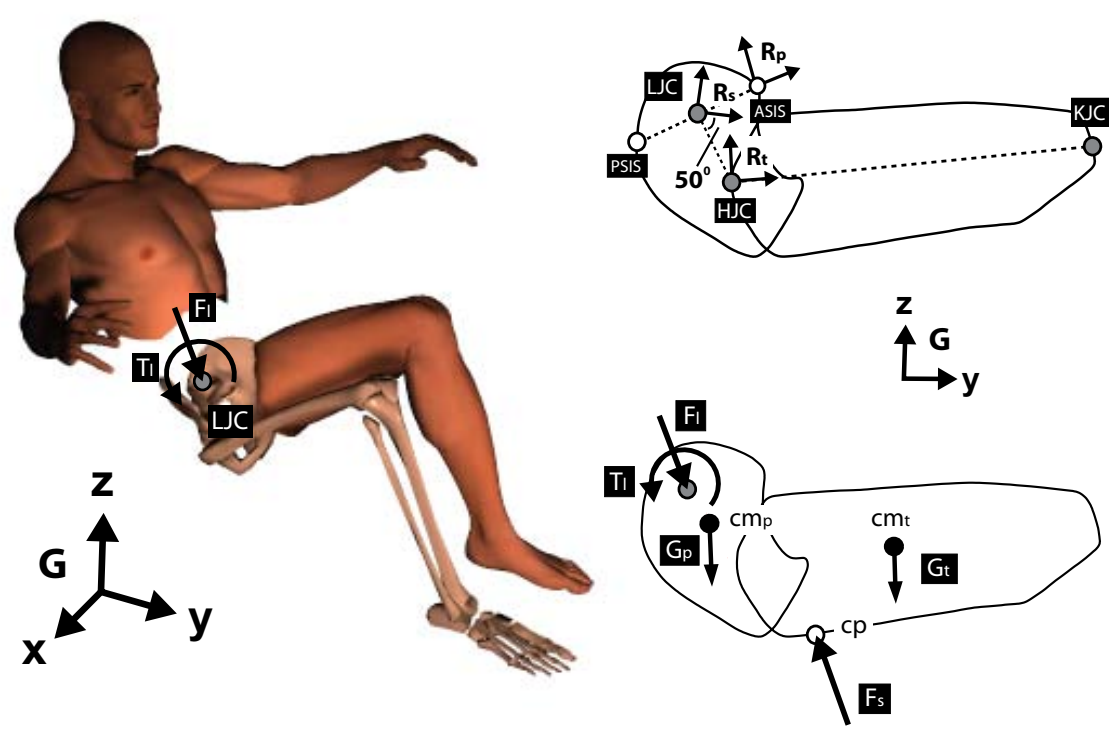

Figure 8.4: A sitting posture with the net joint forces $\left(\mathrm{F}_{1}\right)$ and torques $\left(\mathrm{T}_{1}\right)$ that exert in the lumbosacral joint centre (LJC). The local frames for the sacrum $\left(R_{s}\right)$, pelvis $\left(R_{p}\right)$ and thighs $\left(R_{t}\right)$ are constructed from PSIS, ASIS, LJC, HJC and KJC as shown. The analogue force data was used to calculate centre of pressure $(\mathrm{cp})$ and the seat resultant force $\left(\mathrm{F}_{\mathrm{s}}\right) . \mathrm{F}_{1}$ and $\mathrm{T}_{1}$ are computed relative to LJC using static equations of equilibrium for the pelvis and thighs. 
over a range of $30^{\circ}\left(25 \pm 15^{\circ}\right)$ relative to the seat support. For the second trial, $\beta$ was fixed on $20^{\circ}$ relative to the seat support. Periodic quasi-static $(1 / 30 \mathrm{~Hz})$ ischial buttock pressure relief was induced from downward sinusoid tuberal support adjustment (TSA) over a range of $40 \mathrm{~mm}(-20 \pm 20 \mathrm{~mm})$ below the seating surface. Each trial started with a static reference measurement to define the initial pelvis angle $\left(\alpha_{0}\right)$ and to relate the local sensor frame $\left(\mathrm{R}_{\mathrm{i}}\right)$ to the local pelvis frame $\left(\mathrm{R}_{\mathrm{p}}\right)$.

Subjects were asked to undergo chair movements in a passive way. This meant that they were not allowed to perform any activity that could influence body movement as induced by the chair. Each trial took approximately three minutes of cyclic chair adjustments from which data was only collected during the last 90 seconds. A typical example of measured $\left(\beta, \alpha, \mathrm{cp}, \mathrm{F}_{\mathrm{t}}, \mathrm{BF}_{\mathrm{sc}}\right.$ and $\left.\mathrm{SO} 2_{\mathrm{sc}}\right)$ and estimated $\left(\mathrm{F}_{\mathrm{ly}}, \mathrm{F}_{\mathrm{lz}}, \mathrm{T}_{\mathrm{l}}\right)$ variables during DPR is shown in figure 8.5.

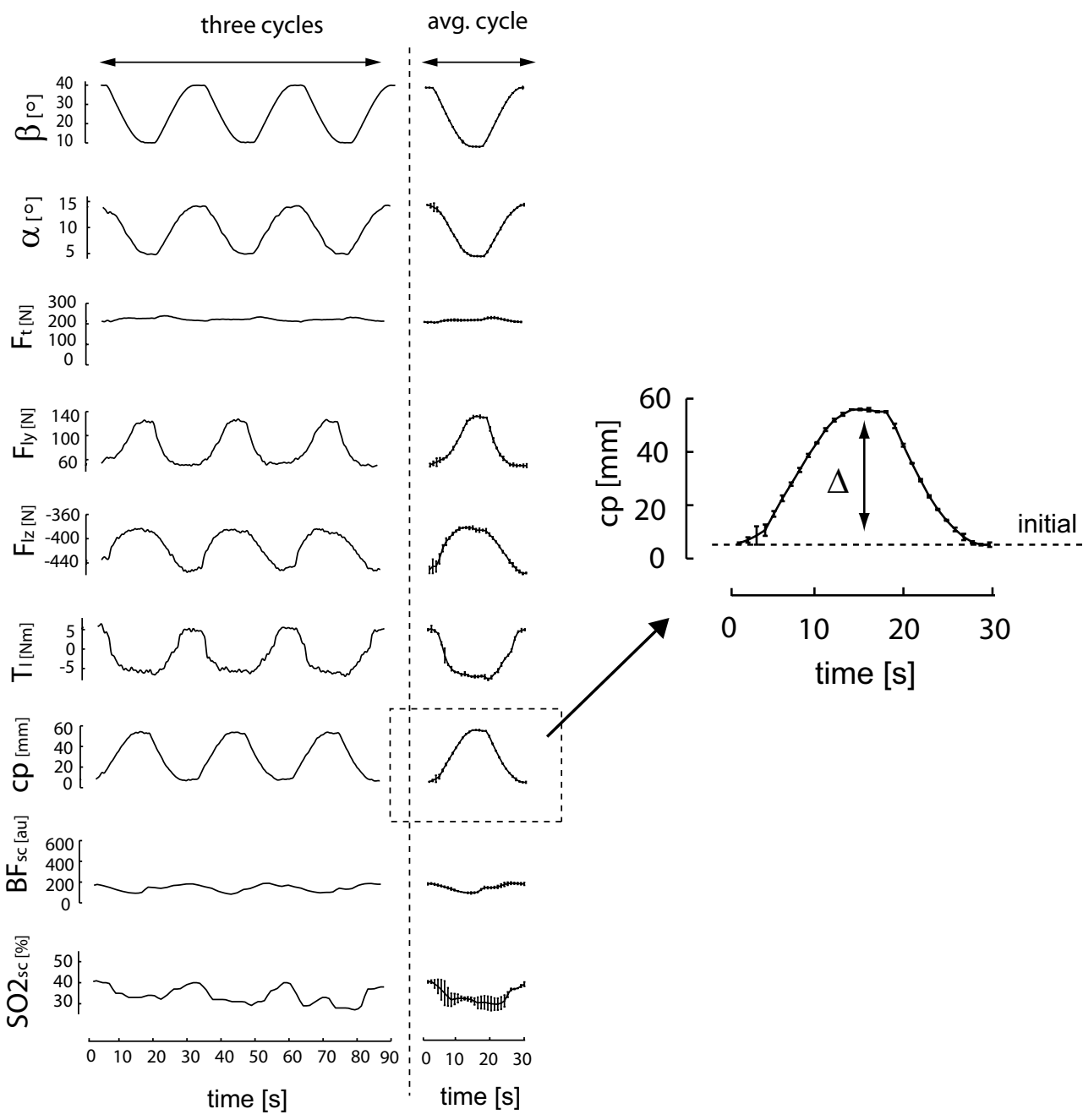

Figure 8.5: A typical example of measured $\left(\beta, \alpha, \mathrm{cp}, \mathrm{F}_{\mathrm{t}}, \mathrm{BF}_{\mathrm{sc}}\right.$ and $\left.\mathrm{SO} 2_{\mathrm{sc}}\right)$ and estimated $\left(\mathrm{F}_{\mathrm{ly}}, \mathrm{F}_{\mathrm{lz}}, \mathrm{T}_{\mathrm{l}}\right)$ variables during DPR. 


\subsubsection{Statistics}

Linear effects of chair modification were assumed. In the first trial we related $\alpha, \mathrm{F}_{\mathrm{t}}, \mathrm{cp}$, $\mathrm{F}_{\mathrm{ly}}, \mathrm{F}_{\mathrm{lz}}, \mathrm{T}_{1}, \mathrm{BF}_{\mathrm{sc}}$ and SO2 ${ }_{\mathrm{sc}}$ to angular parallelogram adjustment $(\beta)$ and in the second trial to downward tuberal support element adjustment (D). We then computed the linear best-fit equations together with the coefficients of correlation $(r)$ and accompanying significance (p). Subsequently, the coefficients of determination $\left(\mathrm{r}^{2}\right)$ were calculated which gives an indication about the strength of the relation. High coefficients of determination $\left(\mathrm{r}^{2}>0.8\right)$ indicate strong relations. According to figure 8.5, the initial value (init), range $(\Delta)$ and coefficient of determinations $\left(\mathrm{r}^{2}\right)$ were computed for both trials and averaged over all subjects. Furthermore, a multiple comparison one-way ANOVA was performed to compare the ranges $(\Delta)$ of all quantities between DPR and TSA.

\subsubsection{Results}

Table 8.1 and 8.2 show the initial values (initial \pm sd), ranges $(\Delta \pm$ sd), coefficients of correlation $(\mathrm{r} \pm \mathrm{sd})$ and coefficients of determination $\left(\mathrm{r}^{2} \pm \mathrm{sd}\right)$ for all quantities in DPR and TSA respectively. Note that the number of subjects is different between interventions because in some trials we encountered problems with the position and visibility of the pelvis mold.

Furthermore, three out of ten subjects showed no response in buttock tissue oxygenation and perfusion $\left(\mathrm{SO}_{\mathrm{sc}}\right.$ and $\mathrm{BF}_{\mathrm{sc}}$. This group was excluded from analysis because the average values for $\mathrm{SO}_{\mathrm{sc}}$ and $\mathrm{BF}_{\mathrm{sc}}$ during TSA were not significantly different than the initial values. In DPR the pelvis rotated $10.9 \pm 2.6$ degrees. High values of $\mathrm{r}^{2}(>0.8)$ were found for $\alpha, \mathrm{cp}$ and $\mathrm{T}_{1}$. In TSA, the pelvis rotated $1.5 \pm 0.8$ degrees. High values of $\mathrm{r}^{2}$ were found for $\mathrm{F}_{\mathrm{t}}, \mathrm{cp}, \mathrm{F}_{\mathrm{ly}}$ and $\mathrm{T}_{\mathrm{l}}$. Grouped in bar diagrams, figure 8.6 compares the amount of change $(\Delta \pm$ sd) for all quantities between DPR and TSA. It shows that $\Delta \alpha, \Delta \mathrm{cp}, \Delta \mathrm{F}_{\mathrm{ly}}, \Delta \mathrm{F}_{\mathrm{lz}}, \Delta \mathrm{T}_{1}$ and $\Delta \mathrm{BF}_{\mathrm{sc}}$ are significantly $(\mathrm{p}<.05)$ larger in DPR, and that $\Delta \mathrm{F}_{\mathrm{t}}$ is significantly larger in TSA.

\subsection{Discussion}

This study investigated the contribution of decoupled pelvis rotation (DPR) and tuberal support adjustment (TSA) on pelvis alignment, low back load, external buttock load and subtuberal blood supply in ten male SCI-subjects. We evaluate how these quantities can be regulated in prolonged static sitting and we compare the outcomes with our findings in able-bodied subjects from previous experiments.

\subsubsection{Pelvis alignment}

We found that DPR rotated the pelvis approximately 11 degrees in sagittal direction. This significant influence on pelvis alignment suggests that this technique is applicable to change pelvis posture is prolonged static sitting. High coefficients of determination indicated a good relation between parallelogram configuration and pelvis alignment. However, relatively large variation between subjects for the initial pelvis angle and range of pelvis rotation make it hard to generalise this relation. 
Table 8.1: Initial values (initial $\pm \mathrm{sd}$ ), ranges $(\Delta \pm \mathrm{sd})$ and coefficients of determination $\left(\mathrm{r}^{2} \pm \mathrm{sd}\right)$ for all measured and estimated quantities in DPR.

\begin{tabular}{lllll}
$\mathrm{DPR}[\mathrm{n}=7]$ & initial $\pm \mathrm{sd}$ & $\Delta \pm \mathrm{sd}$ & $\mathrm{r}^{2} \pm \mathrm{sd}$ \\
\hline $\mathrm{D}$ & $\mathrm{mm}$ & $0 \pm 0$ & $0 \pm 0$ & - \\
$\beta$ & $\mathrm{deg}$ & $40 \pm 0$ & $30 \pm 0$ & - \\
$\alpha$ & $\mathrm{deg}$ & $18.8 \pm 4.0$ & $10.9 \pm 2.6$ & $\mathbf{0 . 9 5} \pm \mathbf{0 . 0 5 *}$ \\
$\mathrm{F}_{\mathrm{t}}$ & $\mathrm{N} / \mathrm{kg}$ & $2.39 \pm 0.33$ & $0.34 \pm 0.20$ & $0.62 \pm 0.35 *$ \\
$\mathrm{cp}$ & $\mathrm{mm}$ & $9.5 \pm 17.8$ & $42.5 \pm 9.8$ & $\mathbf{0 . 9 7} \pm \mathbf{0 . 0 2} *$ \\
$\mathrm{~F}_{\mathrm{ly}} \neq$ & $\mathrm{N} / \mathrm{kg}$ & $0.37 \pm 0.25$ & $0.81 \pm 0.25$ & $0.74 \pm 0.28 *$ \\
$\mathrm{~F}_{\mathrm{lz}} \neq$ & $\mathrm{N} / \mathrm{kg}$ & $-4.32 \pm 1.21$ & $0.70 \pm 0.25$ & $0.79 \pm 0.10 *$ \\
$\mathrm{~T}_{\mathrm{l}} \neq$ & $\mathrm{Nm} / \mathrm{kg}$ & $0.27 \pm 0.18$ & $0.14 \pm 0.04$ & $\mathbf{0 . 8 2} \pm \mathbf{0 . 0 9} *$
\end{tabular}

Subcutaneous blood supply [n $=7]$

\begin{tabular}{|c|c|c|c|c|}
\hline $\mathrm{BF}_{\mathrm{sc}}$ & $\mathrm{au}$ & 142.449 .1 & $94.6 \quad 14.4$ & $0.26 \pm 0.30$ \\
\hline $\mathrm{SO} 2_{\mathrm{sc}}$ & $\%$ & $45.6 \pm 24.6$ & $5.6 \pm 4.2$ & $0.40 \pm 0.37$ \\
\hline
\end{tabular}

bold values for $\mathrm{r}^{2}$ involve a strong relation $\left(\mathrm{r}^{2}>0.8\right)$ with $\beta$

* significant correlation $(\mathrm{p}<.05) ;{ }^{*}$ estimated quantity

Table 8.2: Initial values (initial $\pm \mathrm{sd})$, ranges $(\Delta \pm \mathrm{sd})$ and coefficients of determination $\left(\mathrm{r}^{2} \pm \mathrm{sd}\right)$ for all measured and estimated quantities in TSA.

\begin{tabular}{lllll}
\multicolumn{2}{l}{ TSA $[\mathrm{n}=9]$} & initial $\pm \mathrm{sd}$ & $\Delta \pm \mathrm{sd}$ & $\mathrm{r}^{2} \pm \mathrm{sd}$ \\
\hline $\mathrm{D}$ & $\mathrm{mm}$ & $0 \pm 0$ & $40 \pm 0$ & 1 \\
$\beta$ & $\mathrm{deg}$ & $20 \pm 0$ & $0 \pm 0$ & - \\
$\alpha$ & $\mathrm{deg}$ & $11.3 \pm 4.8$ & $1.5 \pm 0.8$ & $0.79 \pm 0.22 *$ \\
$\mathrm{~F}_{\mathrm{t}}$ & $\mathrm{N} / \mathrm{kg}$ & $3.23 \pm 0.53$ & $3.23 \pm 0.51$ & $\mathbf{0 . 9 5} \pm \mathbf{0 . 0 2} *$ \\
$\mathrm{cp}$ & $\mathrm{mm}$ & $33.6 \pm 13.0$ & $7.3 \pm 3.4$ & $\mathbf{0 . 8 3} \pm \mathbf{0 . 1 3} *$ \\
$\mathrm{~F}_{\mathrm{ly}}{ }^{*}$ & $\mathrm{~N} / \mathrm{kg}$ & $0.79 \pm 0.39$ & $0.19 \pm 0.12$ & $\mathbf{0 . 8 2} \pm \mathbf{0 . 1 5} *$ \\
$\mathrm{~F}_{\mathrm{lz}}{ }^{*}$ & $\mathrm{~N} / \mathrm{kg}$ & $-4.04 \pm 0.94$ & $0.21 \pm 0.11$ & $0.44 \pm 0.27$ \\
$\mathrm{~T}_{1}^{\ddagger}$ & $\mathrm{Nm} / \mathrm{kg}$ & $0.09 \pm 0.22$ & $0.09 \pm 0.03$ & $\mathbf{0 . 9 4} \pm \mathbf{0 . 0 3 *}$
\end{tabular}

Subcutaneous blood supply [n $=7]$

$\begin{array}{lllll}\mathrm{BF}_{\mathrm{sc}} & \mathrm{au} & 80.7 \pm 38.6 & 243.0 \pm 116.2 & 0.77 \pm 0.25 \text { * } \\ \mathrm{SO}_{\mathrm{sc}} & \% & 48.5 \pm 18.0 & 16.8 \pm 14.7 & 0.62 \pm 0.28 \text { * }\end{array}$

bold values for $\mathrm{r}^{2}$ involve a strong relation $\left(\mathrm{r}^{2}>0.8\right)$ with $\mathrm{D}$

* significant correlation $(\mathrm{p}<.05) ;{ }^{*}$ estimated quantity 

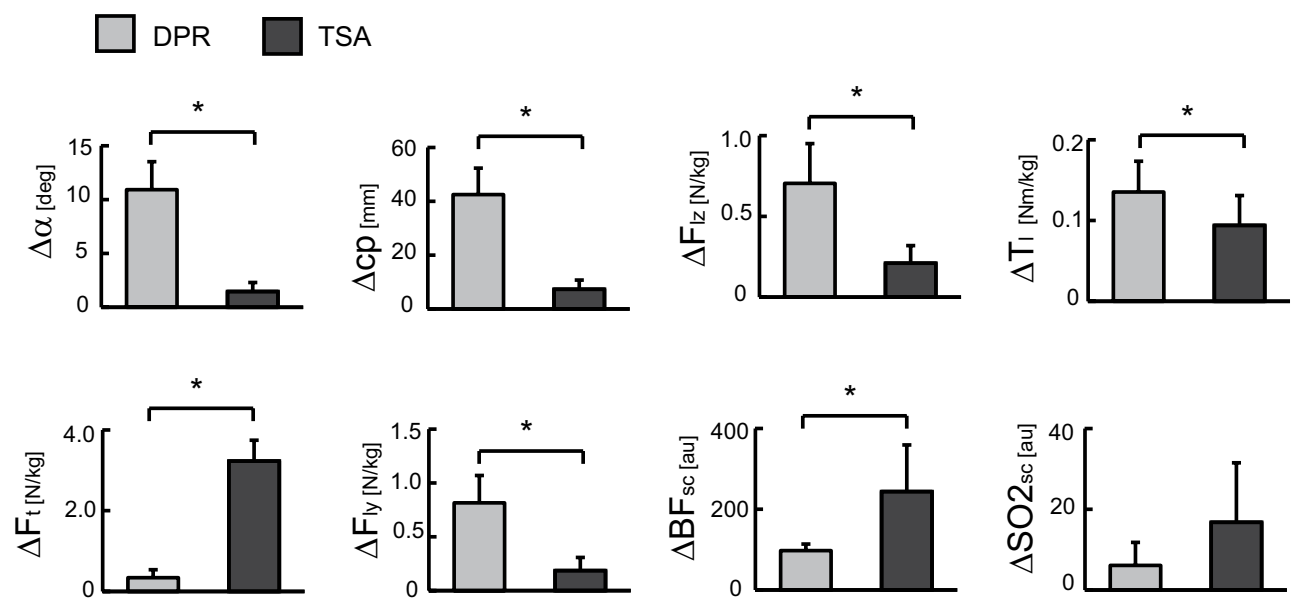

Figure 8.6: Bar diagrams that compares the amount of change ( $\Delta \pm \mathrm{sd}$ ) for all quantities between DPR and TSA; *significant different $(\mathrm{p}<.05)$.

For proper pelvis control, a reference measurement might therefore be necessary to derive the relation per individual in advance.

Although significant effects on pelvis alignment were found, we observed that approximately $1 / 3$ of the adjusted parallelogram angle resulted in pure pelvis rotation. This is reflected in table 8.2 , which shows that $30^{\circ}$ parallelogram angle adjustment $(\Delta \beta)$ causes $10.9^{\circ}$ pelvis rotation $(\Delta \alpha)$. In able-bodied subjects, the effect on pelvis alignment was much larger. We ascribe this to differences in pelvis mold positioning. Although we did our very best to position the mold carefully around the iliac crest, a surplus of superficial body tissue in the pelvis region for most subjects made it very hard to align the mold properly. In some subjects, the extent of pelvis rotation was therefore underestimated. In skinny subjects, however, the mold moved along with the pelvis quite well and resulted in similar outcomes to what we found in able-bodied subjects.

Contrary to what we expected, little effect on pelvis alignment was found during TSA. We observed that the buttock sank into the seating surface when the tuberal support elements were lowered completely. Because the position and alignment of the pelvis changes slightly during TSA, this technique might therefore not only influence the forces under the ischial buttock region but has some secondary effects on spinal posture and load as well.

\subsubsection{Buttock load and subtuberal blood supply}

A strong linear effect of DPR on centre of pressure (cp) was found in all subjects. We explain this by the fact that the position of the backrest moves along when the pelvis rotates in sagittal direction. Because cp follows the upper body centre mass $(\mathrm{cm})$ during pelvis movement, a close relation between cp and DPR was observed. Minimal effect was found for the contact forces in the tuberal region. Because the pelvis rotates roughly around an axis under the ITs during DPR, the tuberal contact forces remain high during the whole adjustment range. However, similar to what we suggested for 
able-bodied subjects, we do consider DPR effective to redistribute buttock load in the sacral region. When the pelvis rotates anteriorly and the sacrum comes loose from the seat support, the external buttock load in this region relieves substantially and travels in forward direction where it distributes under the thighs. Because DPR hardly affects the buttock load in the tuberal region, additional adjustment of the tuberal supports might be essential to alleviate the subtuberal buttock tissue and to maintain tissue perfusion in static sitting.

When the tuberal support elements lowered during TSA we observed that the contact load within this region diminished completely and that the levels of subtuberal tissue perfusion and oxygenation improved significantly. This suggests that a combination of periodic DPR and TSA is applicable to redistribute the external buttock load which potentially benefits the viability of the internal buttock tissue within the whole ischial region.

However, three out of ten subjects showed no significant response in buttock tissue perfusion and oxygenation, and were classified as non-responding subjects. Similar non-responding patterns were reported in a by Bader et al. (Bader, 1990) who investigated the effects of dynamic cushions on skin perfusion in healthy and impaired subjects. They reported that the non-responding group might be subjected to an increased risk for tissue ischemia due to a potential impaired physiological control mechanism. Another explanation might be that for some individuals the external tuberal load involves tissue deformation that does not affect blood flow in the tissue under the measurement probes. Because the stagnation of blood flow might shift to other tissue regions, we expect that periodic relief of the external buttock load is always needed, even when no significant effect on oxygenation and perfusion has been found.

\subsubsection{Low back load}

As an indication for the mechanical conditions of the lumbosacral spine region during intervention, we evaluated the net joint forces $\left(\mathrm{F}_{\mathrm{ly}}\right.$ and $\left.\mathrm{F}_{\mathrm{lz}}\right)$ and torque $\left(\mathrm{T}_{1}\right)$ that acted between the sacral endplate and the fifth lumbar vertebra. We associate $\mathrm{T}_{1}$ and $\mathrm{F}_{\mathrm{lz}}$ with intervertebral disc compression, and $\mathrm{F}_{\mathrm{ly}}$ with the amount of shear. For all three quantities, the effect was significantly larger in DPR. Because the location of the socalled lumbosacral 'joint' centre (LJC) was estimated from the reflective markers on the pelvis mold, inaccurate mold positioning and movement could misinterpret the forces at the lower back. We therefore believe that we underrated the actual influence of DPR due to the observation that in some subjects the movement of the mold was smaller than the movement of the pelvis.

Although expected otherwise, we observed some influence of TSA on the forces at the lower back. We ascribe this to small adaptations of cp and the position and alignment of the pelvis when the tuberal support elements move up and down. We therefore conclude that this technique not only influences the forces under the ischial buttock region but has some consequences for spinal posture and load as well.

Besides the potential benefit for wheelchair-users who do not have the strength to reposition themselves physically, DPR might also be applicable in areas other than wheelchair technology. For instance, low back pain is also a big concern in ablebodied individuals who do sedentary work and sit in confined settings for hours. It 
has been reported that the passive flexion stiffness in moderate ranges of lumbar flexion already increases within two hours of static sitting (Beach et al., 2005). Some researchers (Beach et al., 2005, Parkinson et al., 2004) associated this with an increase in passive resistance of muscles at the lower back and suggested that these could be injured when full lumbar flexion tasks are performed after prolonged static sitting with flexed spinal posture. Therefore, the introduction of DPR in sedentary occupations such as office-workers and car drivers might be applicable to induce sufficient lumbar motion and potentially reduce the risk for low back injury when sitting in confined settings for extended periods of time.

We are well aware that it is very hard to interpret and translate these findings directly to the onset of lumbar discomfort because we did not measure the mechanical and/ or physiological spine conditions at inter-segmental level. Although we consider this part of the study as explorative, we do think that it concerns valuable information about the response of the lower spine and how periodic DPR and TSA could reduce the feeling of lumbar discomfort in prolonged static sitting.

\subsection{Conclusions}

Our findings suggest that DPR is an effective passive motion technique which could periodically rotate the pelvis independent from the trunk and seat support, alleviate the ischial buttock tissue under the sacrum and regulate the forces that exert at the lower back. Additionally, TSA seems applicable to redistribute the external buttock load under the ITs and manipulate the levels of oxygenation and perfusion in the sub-dermal tissue. Because the supply of blood to the subtuberal tissue was inversely related to the forces on the tuberal supports, we believe that periodic TSA potentially benefits the viability of the internal buttock tissue under the ITs. Altogether, we conclude that a combination of both interventions might be useful for simultaneous regulation of body load associated with low back pain and pressure ulcers in impaired individuals who cannot physically reposition themselves in daily wheelchair-use.

\subsection{Acknowledgements}

We would like to thank the engineering company Demcon (Oldenzaal, the Netherlands) for developing the experimental simulator chair. We also thank the nurses from the Roessingh Rehabilitation Centre for their effort during the experiments and all patients from their cooperation. 


\section{Chapter 9}

\section{Decoupled Pelvis Alignment and Functional Movement in Spinal Cord Injured Individuals}

Submitted as: P. van Geffen, J. Reenalda, G.J. Snoek, P.H. Veltink and H.F.J.M. Koopman. Decoupled pelvis alignment affects sitting balance and functional movement in spinal cord injured individuals. Under review. 


\begin{abstract}
This chapter investigates the effects of so-called decoupled pelvis alignment on sitting balance and functional movement in subjects with a spinal cord injury (SCI).

It involves a cross-sectional group study in a rehabilitation centre with ten male SCIsubjects. The ASIA Impairment Scale (AIS) was used to differentiate between subjects groups. The AB-group $(n=6)$ represents subjects with no motor-function below lesion level, and the $C$-group $(n=4)$ represents subjects with reduced motor-function below lesion level. The study uses an adjustable simulator chair with parallelogram design to decouple the alignment of the pelvis from the backrest and seat support. Unsupported bimanual forward reaching tasks were performed in different chair configurations. P130, P120, P110 and P100 defined chair configurations with reflecting pelvis angles of $130^{\circ}, 120^{\circ}, 110^{\circ}$ and $100^{\circ}$ respectively and were set in random order. In all trails, the maximal unsupported reaching distance $\left(h_{m}\right)$, initial centre of pressure position $\left(c p_{0}\right)$, maximal centre of pressure position ( $\left.c p_{m}\right)$ and maximal centre of pressure displacement $(\Delta c p)$ were computed.

For both groups, $c p_{0}$ was significantly $(p<0.05)$ larger in P100 compared to P130. For the AB-group, $h_{m}, c p_{m}$ and $\Delta c p$ were larger in P100 compared to P120 and P130. For the C-group, $\Delta c p$ was larger in P130 compared to P100.

Although the effects were different between subject groups, we conclude that decoupled pelvis alignment affects balance control in SCI-individuals and that anterior tilted pelvis postures potentially benefit the functional performance in daily wheelchair-use.
\end{abstract}




\subsection{Introduction}

Functional balance during unsupported movement of the upper body relies on an adequate neuromuscular proprioceptive response that keeps the upper body centre of mass within specific stability limits. An impaired neuromuscular function of the trunk and lower extremities makes therefore that many wheelchair-users suffer postural instability and have problems with functional movement tasks during activities of daily life (Chen et al., 2003, Curtis et al., 1995, Lanzetta et al., 2004, Seelen et al., 2001, Janssen-Potten et al., 1999).

In this paper, postural control and balance control are equivalent and both refer to the possibility to maintain posturally stable during internal and external perturbation. For seating conditions that allow large perturbation before reaching the boundaries of postural instability, very little postural control is needed to maintain posturally stable, but at the same time, large effort is needed to change body posture for functional purpose. Seating conditions at the edge of postural instability, however, needs better postural control to maintain stability and requires less effort to change body posture functionally.

To increase the base of support and to bring the body centre of mass closer the support surface, individuals who lack postural control often adopt a kyphotic spinal posture with posterior tilted pelvis (Hobson and Tooms, 1992, Koo et al., 1996). Some individuals even recline the seat support and squeeze the pelvis and lower spine against the backrest for extra postural stability during upper extremity movement tasks (Maurer and Sprigle, 2004). Clinical observations have indicated that many impaired individuals 'feel' more stable when sitting with posterior tilted pelvis (Maurer and Sprigle, 2004). However, such flexed spinal postures have negative influence on body movement in forward direction (Faiks and Reinecke, 1998). Because the motion of the spine is constrained between the posterior tilted pelvis and the backrest, the ability to reach forward is limited to the shoulder girdle only.

Regarding the functional independence of wheelchair-users, important criteria for new wheelchair design involve interventions that provide postural stability without constraining functional movement. The ability to balance the trunk during unsupported body movement has therefore often been analysed (Aissaoui et al., 2001, Sprigle et al., 2003, Lanzetta et al., 2004, Powell et al., 2002, Seelen et al., 1997, Janssen-Potten et al., 1999). However, few studies are known that evaluated wheelchair adaptations which potentially improve the performance of functional movement tasks (Janssen-Potten et al., 2001, Janssen-Potten et al., 2000, JanssenPotten et al., 2002, Curtis et al., 1995).

In their search to find a trade-off between postural stability and freedom of trunk and upper extremity movement, Janssen-Potten et al. evaluated sitting balance in spinal cord injured (SCI) individuals by measuring reaching distance and centre of pressure displacement for different chair modifications during an unsupported bimanual forward reaching task (Janssen-Potten et al., 2000). When comparing the effects of chair recline (tilt-in-space) and backrest recline with a standard chair configuration, an improved sitting balance was found in low thoracic SCI-individuals. In high thoracic SCI-individuals, chair modifications did not influence balance control.

Because the pelvis forms the basis for trunk support and directly affects the curvature of the lumbar spine, seating interventions that adjust pelvis angle in 
sagittal direction might be applicable to regulate spinal posture and to improve the functional performance in daily wheelchair-use. Forward seat inclination has sometimes been suggested as a useful technique to rotate the pelvis in anterior direction and to enhance a lordotic curvature of the lower spine when individuals lack muscle function for active control of pelvis position and spinal posture. With this in mind, Janssen-Potten investigated the effects of 10 degrees forward seat inclination on pelvis alignment and balance control in thoracic SCI-individuals (Janssen-Potten et al., 2001). No influence on pelvis angle and balance control was observed. It was suggested that anterior pelvis rotation potentially jeopardizes sitting balance during forward seat inclination and that SCI-individuals maintain a posterior tilted pelvis posture to benefit the stabilising configuration of the back support. Because all body adaptation occurred in the hip joints, spinal posture was not affected and sitting balance did not change due to minimal alteration in the base of support.

In a recent study, a seating intervention was introduced that uses a parallelogram design to impose sagittal pelvis rotation from adjusting the position of the backrest (van Geffen et al., 2008b). Although the effects on pelvis alignment have been demonstrated (van Geffen et al., 2008a), no clinical investigation has yet been performed on postural control. Alignment of the chair pivots with the anatomical axes for pelvis rotation allows sagittal adjustment of pelvis angle independent from the orientation of the backrest and seat support. Anterior pelvis rotation due to forward backrest adjustment decreases the base of support and potentially jeopardises sitting balance. However, when the buttock stays in place and the backrest moves forward relative to the seat support, the pelvis gets stabilised between the thighs and trunk. Theoretical evaluation of the static requirements for trunk stabilisation in sitting has shown that for chair adaptations which increase the stiffness of pelvis stabilisation less lumbar stiffness is required to maintain postural stability(Bidard et al., 2000). This suggests that individuals who lack trunk muscle function might benefit from stiff pelvis stabilisation when the base of support decreases and sitting balance gets jeopardised in anterior tilted pelvis postures.

The present study uses a simulator chair with parallelogram design that adjusts the backrest in sagittal direction and rotates the pelvis segment independent from the thighs and trunk. Based on the performance of controlled bimanual forward reaching tasks, we investigate the effects of so-called decoupled pelvis alignment on balance control and functional movement in SCI-individuals. Because the position of the backrest affects the base of body support, pelvis alignment and the stiffness of pelvis stabilisation, we expect significant influence on balance control and that anterior tilted pelvis postures improves the ability to reach forward.

\subsection{Methods}

\subsubsection{Subjects}

Ten male subjects (age $57.6 \pm 7.4$ years, weight $87.3 \pm 14.1 \mathrm{~kg}$, length $1.80 \pm 0.07 \mathrm{~m}$ ) with an impaired neuromuscular function of the trunk and lower extremities due to a spinal cord injury (SCI) were recruited for this study. Based on the international standards for neurological and functional classification of spinal cord injury (published by the American Spinal Injury Association, ASIA), the ASIA Impairment 
Scale (AIS) was used to differentiate between SCI-subjects(American Spinal Injury Association, 2000). Subjects were graded with A or B when no motor-function was preserved below lesion level, and with $\mathrm{C}$ when some motor-function was preserved below lesion level. As shown in table 9.1, six subjects (\#1 - \#6) were graded with A or B, and three subjects (\#7 - \#9) were graded with C. Subject \#10 did not suffer a spinal cord lesion but experienced similar neuromuscular symptoms as those who were graded with $C$. Note that the level of lesion is different between subjects. Because the diversity in preserved muscle functioning can be remarkable between similar levels of lesion, distinction between groups was based on the AIS-ratings instead. Subjects (\#1 - \#6) who were graded with A or B represent the AB-group, and subjects (\#7 - \#10) who were graded with $\mathrm{C}$ represent the $\mathrm{C}$-group. Before and after the experiment, an experienced nurse carefully checked the ischial buttock region to be sure that there was no skin damage or other signs of pressure sores. In all subjects, no such problems were observed. Furthermore, the study was approved by the local Committee for Medical Ethics and all subjects read and signed an 'informed consent' in which the objective and experimental protocol was explained.

Table 9.1: Subject classification regarding the level and extent of spinal cord injury

\begin{tabular}{|c|c|c|c|c|c|c|c|c|c|c|}
\hline & \multicolumn{6}{|c|}{ AB-group } & \multicolumn{4}{|c|}{ C-group } \\
\hline & $\# 1$ & $\# 2$ & \#3 & $\# 4$ & $\# 5$ & $\# 6$ & $\# 7$ & $\# 8$ & $\# 9$ & $\# 10^{\prime}$ \\
\hline Level & Th12 & Th11 & Th9 & Th5 & Th4 & $\mathrm{C} 5$ & Th10 & Th5 & $\mathrm{C} 4$ & - \\
\hline AIS & A & A & A & A & B & A & $\mathrm{C}$ & $\mathrm{C}$ & C & - \\
\hline
\end{tabular}

AIS $=$ ASIA impairment scale:

$\mathrm{A}=$ Complete lesion with no motor function preserved below lesion level.

$\mathrm{B}=$ Incomplete lesion with no motor function preserved below lesion level.

$\mathrm{C}=$ Incomplete lesion with reduced motor function below lesion level. More than half of the preserved muscles have a muscle grade less than 3 .

* Subject who did not suffer a spinal cord lesion but experienced similar neuromuscular symptoms as those who were graded with $\mathrm{C}$

\subsubsection{Instrumentation}

Experiments have been performed with a computer-aided adjustable simulator chair (fig 9.1) instrumented with a parallelogram design that allows sagittal pelvis rotation independent from the backrest and seat support. When the lower and upper axes for pelvis adjustment align the ischial tuberosities and lumbar region respectively, adjusting the parallelogram configuration causes the pelvis to rotate sagittaly without affecting the orientation of the trunk and thighs. Six uni-axial load-cells were mounted under the seat support for accurate measurement of centre of pressure (cp). An infrared camera motion capturing system was used to capture the position of reflective markers that were placed on a reach-object and the third metacarpal joints of both hands. 

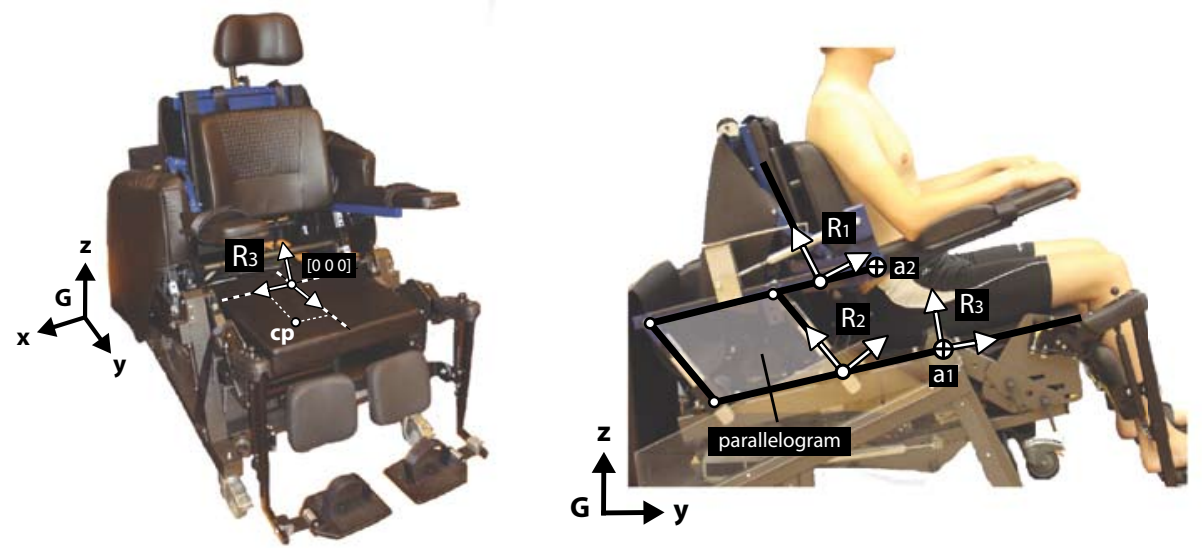

Figure 9.1: Computer-aided adjustable simulator chair with parallelogram design for so-called decoupled pelvis alignment. The lower and upper axes for pelvis rotation (a1 and a2) align the ischial tuberosities and lumbar region respectively. $G$ defines the global reference frame. Six uni-axial load cells that are mounted in the seat support for measurement of centre of pressure (cp). The local coordinate frames for the backrest $\left(\mathrm{R}_{1}\right)$, parallelogram $\left(\mathrm{R}_{2}\right)$ and seat support $\left(\mathrm{R}_{3}\right)$ were constructed from angular chair configurations. Centre of pressure (cp) is expressed in $R_{3}$ relative to the origin $\left[\begin{array}{lll}0 & 0 & 0\end{array}\right]$.

\subsubsection{Experimental setup, chair configurations and reaching task}

Prior to the experiment, an empty seated zero-force calibration measurement was performed. A transfer lift was used to transfer subjects from their wheelchair to the simulator chair. Subjects were seated with their tuberosities on the pivot for sagittal pelvis rotation. The subjects' anatomical sagittal plane aligned the global y-z plane. The footrests were set perpendicular to the seat and the thighs were fully supported except for the popliteal space.

Reflective markers were placed and initial angles for the seat and backrest were set to $10^{\circ}$ and $110^{\circ}$ relative to the horizontal (fig 9.2).

A reach-table was positioned in front of the subject as shown in figure 9.2A. The height of the table was adjusted in a way that the hands were supported and the arms were stretched and lowered $15^{\circ}$ relative to the horizontal.

The experiment involved four trials (fig 9.2), each investigating the effect of parallelogram configuration on the displacement of the hands and cp during a controlled bimanual maximum forward reaching task.

P130, P120, P110 and P100 defined chair configurations with reflecting pelvis angles $(\alpha)$ of $130^{\circ}, 120^{\circ}, 110^{\circ}$ and $100^{\circ}$ respectively and were set in random order. Each reaching task involved a reach procedure of two phases (fig 9.3). The task starts with the reach-object in front of the subject and both hands flat on the table. During the first phase, the subject places the reach-object as far as possible on the table during a controlled bimanual unsupported forward reaching movement and returns to the initial position afterward. During the second phase, the subject reaches forward again and grabs the reach-object with both hands to place it back in the initial position. In each trial, this procedure was repeated five times. Data was collected during the second, third and fourth reach procedure. 


\subsubsection{Data analysis}

The $\mathrm{x}-\mathrm{z}$ and $\mathrm{y}-\mathrm{z}$ planes of the global reference frame $(\mathrm{G})$ were aligned to respectively the frontal and sagittal planes of the simulator chair. The local coordinate frames for the backrest $\left(\mathrm{R}_{1}\right)$, parallelogram $\left(\mathrm{R}_{2}\right)$ and seat support $\left(\mathrm{R}_{3}\right)$ were constructed from angular chair configurations. The origin of $\mathrm{R}_{3}$ lies in the middle of the seat support on top of the axis for sagittal pelvis rotation. Centre of pressure (cp) was expressed relative to origin along the y-axes of $R_{3}$. Hand position (h) was defined from the average of the left and right hand markers. The position of the reach-object (ro) and $\mathrm{h}$ were both expressed relative to the origin along the $y$-axes of $\mathrm{G}$ (fig 9.4).

Figure 9.5 gives a typical measurement of ro, $\mathrm{h}$ and $\mathrm{cp}$ during the second, third and fourth reach procedure. Average values for the maximal reaching distance $\left(\mathrm{h}_{\mathrm{m}}\right)$, initial cp position $\left(\mathrm{cp}_{0}\right)$, maximal cp position $\left(\mathrm{cp}_{\mathrm{m}}\right)$ and maximal cp displacement $(\Delta \mathrm{cp})$ were computed for all trials. A multiple comparison one-way ANOVA was performed to compare the effects on all quantities $\left(\mathrm{h}_{\mathrm{m}}, \mathrm{cp}_{0}, \mathrm{cp}_{\mathrm{m}}\right.$ and $\left.\Delta \mathrm{cp}\right)$ between P130, P120, P110 and P100.
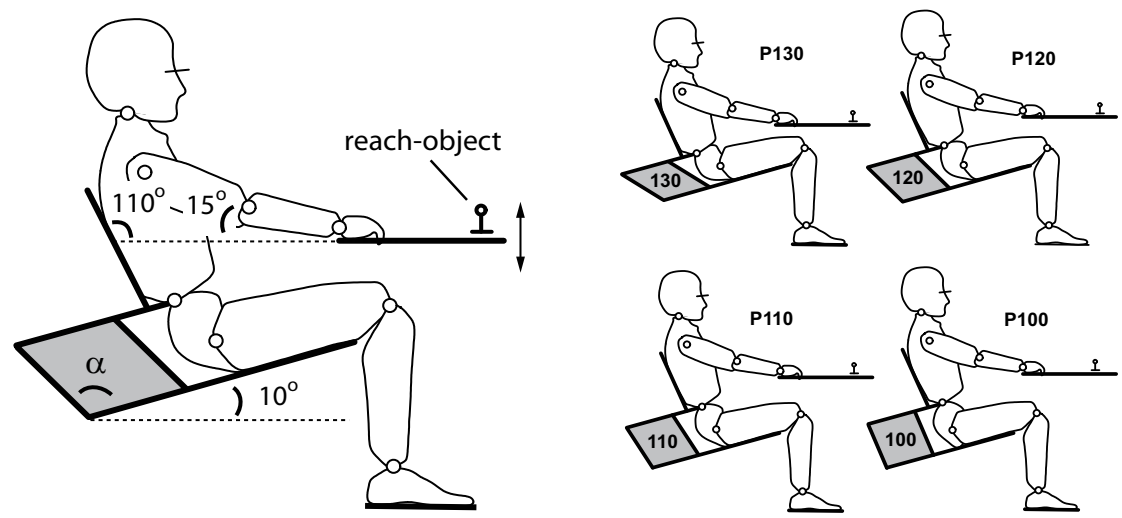

Figure 9.2: Schematic representation of the initial seating conditions. The angle of the seat support, backrest and arms were set $10^{\circ}, 110^{\circ}$ and $15^{\circ}$ relative to the horizontal. P130, P120, P110 and P100 defined chair configurations with reflecting pelvis angles $(\alpha)$ of $130^{\circ}, 120^{\circ}, 110^{\circ}$ and $100^{\circ}$ respectively.

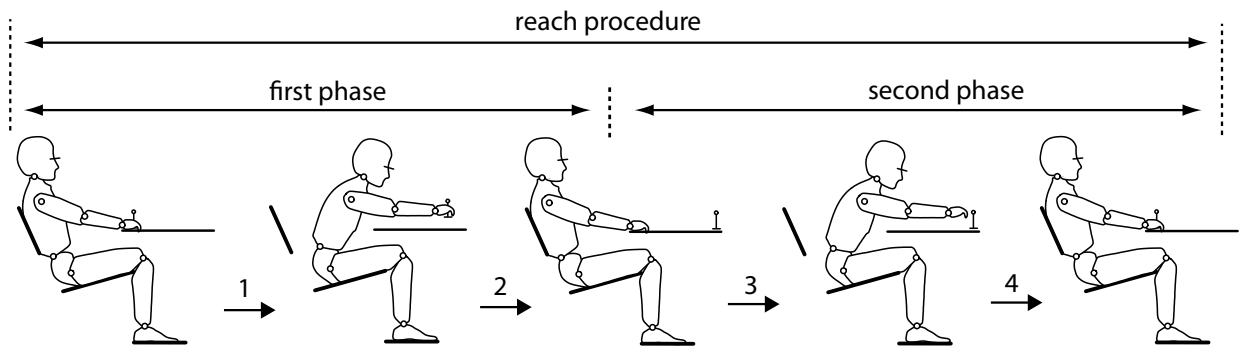

Figure 9.3: The reach procedure involves of two phases and starts with the reach-object in front of the subject and both hands flat on the table. During the first phase, the subject reaches forward to place the reach-object as far as possible (1) and returns to the initial position afterward (2). During the second phase, the subject reaches forward to grab the reach-object (3) and places it back in the initial position (4). 

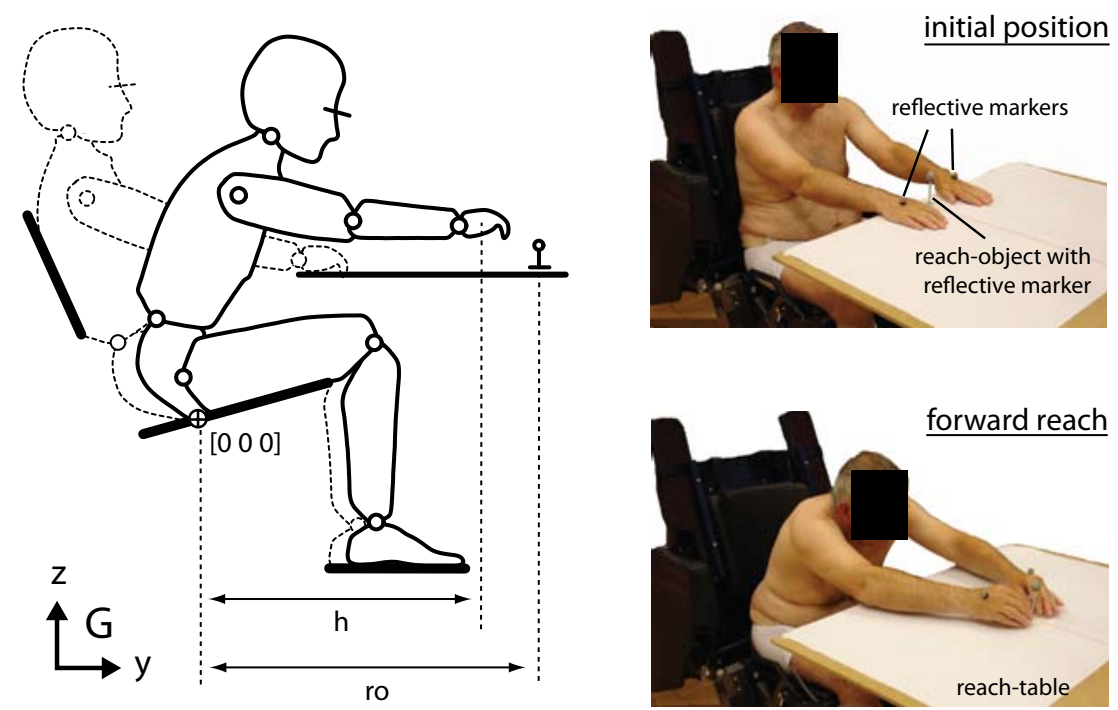

Figure 9.4: Representation of the initial position and unsupported forward reach with reflective markers on the hands and reach-object. The position of the hands (h) and reach-object (ro) were expressed relative to the origin along the y-axis of G.

\subsection{Results}

Mean values of $\mathrm{h}_{\mathrm{m}}, \mathrm{cp}_{0}, \mathrm{cp}_{\mathrm{m}}$ and $\Delta \mathrm{cp}$ were averaged for both groups and presented in table 9.2. To compare the effects between P130, P120, P110 and P100 for the $\mathrm{AB}$-group and the C-group, the means of $\mathrm{h}_{\mathrm{m}}, \mathrm{cp}_{0}, \mathrm{cp}_{\mathrm{m}}$ and $\Delta \mathrm{cp}$ were grouped in bar diagrams as shown in figure 9.6.

\subsubsection{Maximal reaching distance}

For the C-group, no significant differences were found between the effects of chair configuration (P130, P120, P110 and P100) on the maximal unsupported bi-manual reaching distance $\left(\mathrm{h}_{\mathrm{m}}\right)$. For the AB-group, on the other hand, $\mathrm{h}_{\mathrm{m}}$ was significantly $(\mathrm{p}<$ 0.05) larger in P100 compared to P120 and P130.

\subsubsection{Centre of pressure position and displacement}

A significant $(\mathrm{p}<0.05)$ effect of parallelogram configuration on the initial cp position $\left(\mathrm{cp}_{0}\right)$ was found for the C-group and for the AB-group. Both groups showed larger values for $\mathrm{cp}_{0}$ in $\mathrm{P} 100$ compared to P130. Similar to the effects of chair configuration on the maximal reaching distance $\left(\mathrm{h}_{\mathrm{m}}\right)$, the C-group showed no differences on the maximal $\mathrm{cp}$ position $\left(\mathrm{cp}_{\mathrm{m}}\right)$. For the $\mathrm{AB}$-group, we found that $\mathrm{cp}_{\mathrm{m}}$ was significantly smaller in P130 compared to P100 and P110. The effect of parallelogram configuration on the maximum cp displacement $(\Delta \mathrm{cp})$ during unsupported forward reach was opposite between the two subjects groups. Where for the C-group $\Delta \mathrm{cp}$ appeared to be significantly larger in P130 compared to P100, for the AB-group $\Delta$ cp seemed smaller in P130 compared to P100. 
Table 9.2: Average values $( \pm \mathrm{sd})$ of $\mathrm{h}_{\mathrm{m}}, \mathrm{cp}_{0}, \mathrm{cp}_{\mathrm{m}}$ and $\Delta \mathrm{cp}$ in all chair configurations (P130, P120, $\mathrm{P} 110$ and P100) for the C-group and the AB-group.

\begin{tabular}{|c|c|c|c|c|c|c|}
\hline & & & P130 & P120 & P110 & P100 \\
\hline C-group & $\mathrm{h}_{\mathrm{m}}$ & [mm] & $882.9 \pm 74.5$ & $886.8 \pm 59.3$ & $891.3 \pm 43.6$ & $905.9 \pm 48.1$ \\
\hline \multirow[t]{3}{*}[\mathrm{n}=4]{} & $\mathrm{cp}_{0}$ & [mm] & $-9.9 \pm 23.7$ & $1.0 \pm 22.6$ & $19.1 \pm 15.3$ & $41.4 \pm 18.1$ \\
\hline & $\mathrm{cp}_{\mathrm{m}}$ & [mm] & $117.0 \pm 27.9$ & $119.2 \pm 22.3$ & $121.1 \pm 16.4$ & $127.3 \pm 17.7$ \\
\hline & $\Delta \mathrm{cp}$ & [mm] & $126.9 \pm 20.2$ & $118.2 \pm 21.0$ & $101.9 \pm 13.9$ & $85.9 \pm 13.4$ \\
\hline \multirow{4}{*}{$\begin{array}{l}\text { AB-group } \\
{[\mathrm{n}=6]}\end{array}$} & $\mathrm{h}_{\mathrm{m}}$ & [mm] & $507.5 \pm 83.8$ & $564.9 \pm 79.7$ & $628.8 \pm 80.3$ & $719.1 \pm 59.4$ \\
\hline & $\mathrm{cp}_{0}$ & [mm] & $9.5 \pm 8.4$ & $23.0 \pm 7.6$ & $33.7 \pm 11.3$ & $37.7 \pm 10.9$ \\
\hline & $\mathrm{cp}_{\mathrm{m}}$ & [mm] & $5.5 \pm 23.9$ & $30.7 \pm 19.0$ & $53.0 \pm 30.0$ & $81.7 \pm 18.5$ \\
\hline & $\Delta \mathrm{cp}$ & [mm] & $-3.9 \pm 18.6$ & $7.7 \pm 19.0$ & $19.3 \pm 31.6$ & $44.0 \pm 13.7$ \\
\hline
\end{tabular}

\subsection{Discussion}

The present study used an adjustable simulator chair with parallelogram design to investigate the effects of sagittal backrest position on sitting balance and functional movement in SCI-subjects. Based on controlled bimanual forward reaching tasks, our main findings suggest that the position of the backrest influences balance control due to alterations in the base of support, spinal posture and pelvis alignment. Because the diversity in preserved muscle functioning can be remarkable between similar lesion levels, the distinction between subject groups was not based on injury height but on the AIS-ratings instead. Subjects with no motor-function below lesion level were graded with A or B and represented the AB-group. Subjects who still had some motor-function left below lesion level were graded with $\mathrm{C}$ and represented the C-group. We believe that the differences found in reaching behaviour between both subject groups justify this distinction.

A larger displacement of the hands and centre of pressure (cp) in chair configurations with forward backrest position were found for the AB-group. This suggests that anterior tilted pelvis postures benefits the ability to reach forward and is therefore associated with an improved balance control in sitting. For the C-group, the maximal reaching distance and the maximal cp position were not affected. The preserved trunk and pelvis muscle function appeared to be just enough to come loose from the backrest and to reach forward completely in all chair configurations. Because this group could maintain balance during movement with unsupported trunk, the ability to reach forward was not constrained by the position of the backrest.

However, differences in cp displacement were measured. It was found that the maximal displacement of $\mathrm{cp}$ was smaller in anterior tilted pelvis postures which could be associated with a decrease in sitting balance (Janssen-Potten et al., 2000). However, because the maximal $\mathrm{cp}$ position was reached in all configurations we do not ascribe these differences to an alteration in balance control. It merely gives an 


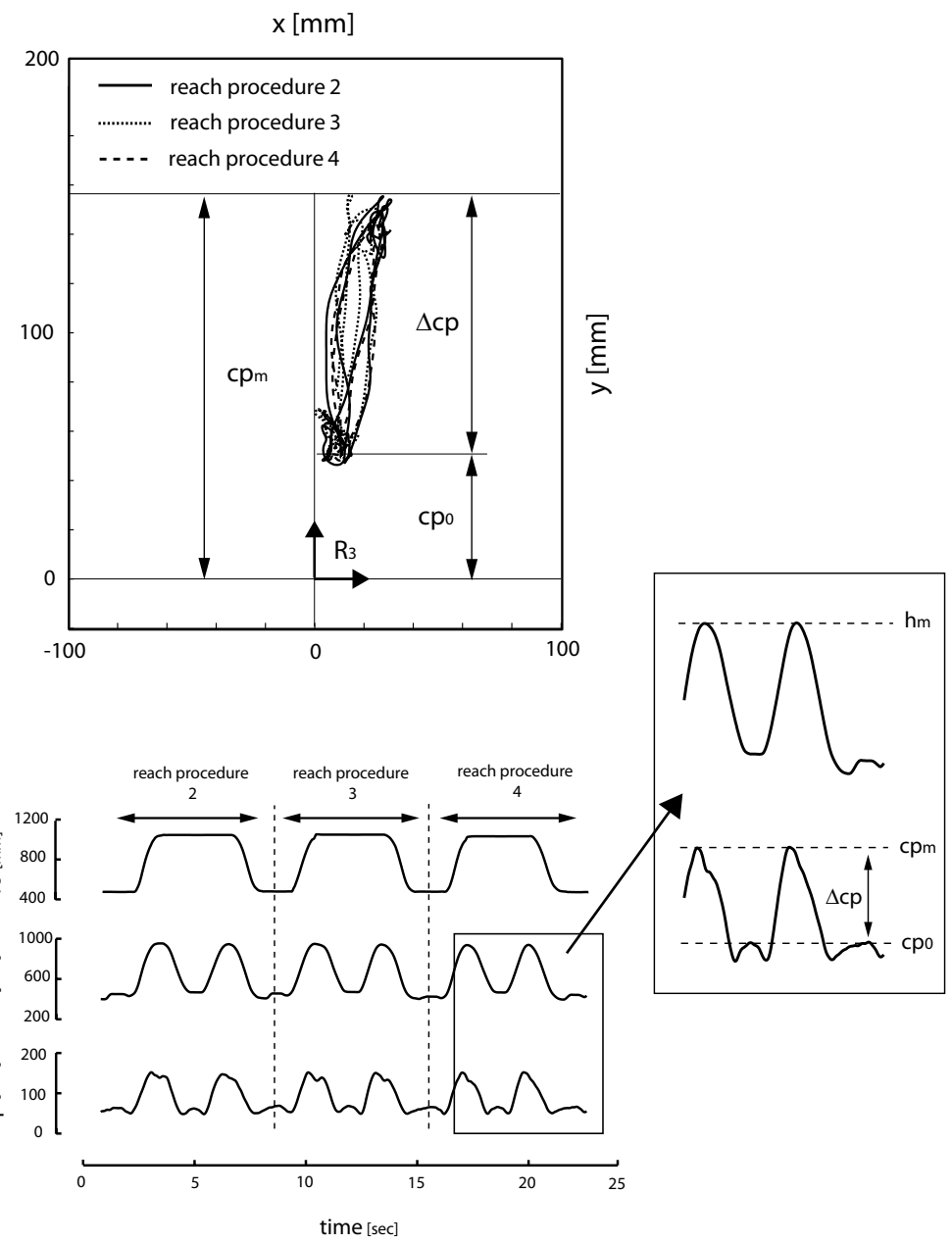

Figure 9.5: The lower figure shows a typical measurement of $\mathrm{cp}, \mathrm{h}$ and ro during three successive reach procedures. The maximal reaching distance $\left(\mathrm{h}_{\mathrm{m}}\right)$, initial $\mathrm{cp}$ position $\left(\mathrm{cp}_{0}\right)$, maximal $\mathrm{cp}$ position $\left(\mathrm{cp}_{\mathrm{m}}\right)$ and maximal $\mathrm{cp}$ displacement $(\Delta \mathrm{cp})$ were computed. The upper figure shows a scan of cp displacement along the $\mathrm{x}$ - and $\mathrm{y}$-axis of $\mathrm{R}_{3}$.

indication about the initial cp position. We found that the begin position of $\mathrm{cp}$ was larger in chair configurations with anterior backrest position. This in combination with unchanged maximal cp position during forward reach simply results that less cp displacement is possible. Despite this reduction in maximal cp displacement, we rather associate anterior tilted pelvis postures with an improved postural control since we observed that less effort was needed to move the upper body in forward direction.

Differences in balance control between the AB-group and the C-group could be ascribed to the extent of preserved pelvis and trunk muscle function but also to the position of the axis for trunk rotation during unsupported forward reach. Subjects in the C-group still had some pelvis muscles left to stabilise the lumbosacral spine 
C-group $[n=4]$
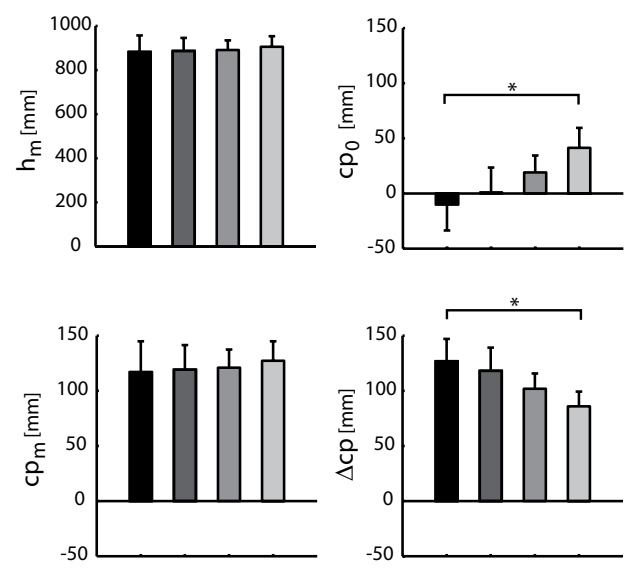

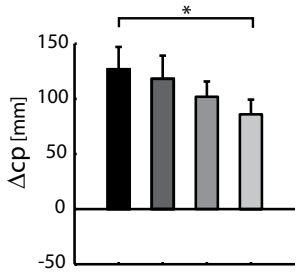

P120

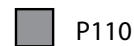

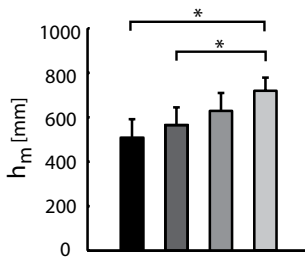
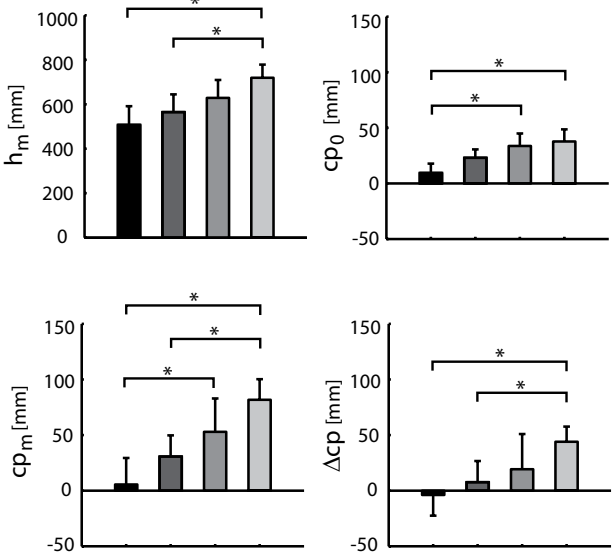

P130

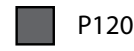

P100

Figure 9.6: Bar diagrams with average values $( \pm \mathrm{sd})$ of $\mathrm{hm}, \mathrm{cp}_{0}, \mathrm{cp}_{\mathrm{m}}$ and $\Delta \mathrm{cp}$ to compare the effects between P130, P120, P110 and P100 for the C-group and AB-group. * significantly different $(\mathrm{p}>0.05)$

region during upper body movement and allows them to rotate the trunk around an axis under the ischial tuberosities (ITs). The ability to reach forward is therefore directly related to the distance between the line of gravity of the upper body and the axis of rotation under the IT. Because the position of the IT does not change between chair configurations, no differences in maximal reaching distance were found. For SCI-subjects with no motor function below lesion level, the axis for trunk rotation shifts to the lumbar spine region due to the lack of preserved pelvis muscle function. They become postural instable much earlier which directly limits their ability to balance the trunk in forward direction.

When the pelvis rotates anteriorly due to forward adjustment of the backrest, the base of support decreases and allows less cp displacement before reaching the boundaries of postural instability. For the AB-group, however, the base of support does not change because the axis for trunk rotation, which lies within in the lumbar spine region, travels along with the upper body centre of mass. How come that the maximal reaching distance and $\mathrm{cp}$ displacement is larger in anterior tilted pelvis postures, even though the distance between the line of gravity and the axis of trunk rotation did not change? We ascribe this to the passive flexion-extension resistance of the lower spine which alters when its sagittal curvature changes. In posterior tilted pelvis postures the lower spine adopts a kyphotic curvature and causes a passive extension torque that forces the trunk against the backrest. Where the C-group have just enough muscle strength to overcome this passive resistance force, the AB-group lacks the strength that is needed to move the trunk in forward direction which results in a minimal reaching distance and cp displacement. Some subjects even showed small displacement of $\mathrm{cp}$ in backward direction because they forced the pelvis posteriorly and tried to gain little reaching distance from flexing the spinal 
curvature even more. When adjusting the backrest position in forward direction, the curvature of the lower spine changes to a lordotic posture with anterior tilted pelvis. Because the line of gravity does not change relative to the base of support, we ascribe the improvement in reaching distance and cp displacement to a decrease in passive resistance against spinal flexion when sitting in an anterior tilted lordotic spinal posture. It was even found that one subject could not maintain postural stability in chair configurations (P100 and P110) with forward backrest position. This indicates that the line of gravity falls outside the base of support and that there is not enough passive spinal resistance to prevent the trunk from falling. In posterior pelvis postures, however, the spine provides more resistance against trunk flexion which could prevent forward trunk instability even when the upper body centre of mass lies anterior of the axis of trunk rotation.

Janssen-Potten (Janssen-Potten et al., 2000) also evaluated the effects of sagittal chair modification on balance control in SCI-subjects. They found that in low thoracic SCI-subjects the ability to shift $\mathrm{cp}$ in forward direction during unsupported reach was larger when reclining the chair (tilt-in-space) or backrest. In high thoracic SCIsubjects however, no significant effect was found. This latter was explained by the fact that in all configurations high thoracic SCI-subjects did not have the strength to counteract the force of gravity that keeps the trunk against the backrest. Because the curvature of the spine does hardly change when reclining the chair or backrest, the passive spinal characteristics could not play a role in the ability to move the trunk functionally. In our study, however, changing pelvis alignment due to sagittal backrest adjustments does affect spinal posture and benefits the ability to reach forward when adopting a body posture with anterior tilted pelvis.

\subsection{Conclusions}

Although minimal effects were found in the maximal reaching distance and cp position for SCI-subjects who still had some motor function left below lesion level, it was observed that more effort is needed to reach forward in posterior tilted pelvis postures. For SCI-subjects with no motor function below lesion level, larger displacement of the hands and cp during unsupported bimanual forward reach suggest that anterior pelvis alignment benefits the ability for functional movement and is therefore associated with an improved balance control in sitting. Although the influence on reaching distance and cp displacement was different between subject groups, we conclude that decoupled pelvis alignment affects balance control in SCIindividuals and that anterior tilted pelvis postures potentially benefit the functional performance in daily wheelchair-use.

\subsection{Acknowledgements}

We would like to thank the engineering company Demcon (Oldenzaal, the Netherlands) for developing the experimental simulator chair. We also thank the nurses from the Roessingh Rehabilitation Centre for their effort during the experiments. 


\section{$\underline{\text { Part V }}$}

\section{General Discussion}




\section{Chapter 10}

\section{General Discussion and \\ Conclusions}




\subsection{Introduction}

Wheelchair-users with reduced postural stability often have problems with the performance of functional movement tasks during activities of daily life. A lack of controlled trunk muscle function makes that many individuals adopt a static body posture which unconditionally leads to all kinds of physical discomfort (e.g. pressure ulcers, posture deformation, joint stiffness, lumbar immobility, low back injury, etc.) in long-term sitting. It has widely been accepted that most physical problems occur from sustained mechanical body loading and that dynamic seating interventions are needed to enhance body movement when individuals do not have the strength to change posture themselves. For individuals who cannot reposition themselves physically, adequate postural variation can only be realised from automatic chair modification or by the help of others. Because the latter often involves a time consuming and labour intensive activity, we tried to enhance the ability for repositioning by adjusting the global configuration of the chair.

\subsubsection{Objectives}

The present thesis described the development of an experimental simulator chair and answered the following research questions:

Q1 How can sitting posture be controlled?

Q2 What interventions are effective to regulate body load associated with physical discomfort (i.e. pressure ulcers and low back pain)?

Q3 What interventions benefit the performance of functional movement in impaired sitting?

Because our research questions (Q1-Q3) cannot be seen separately, they will be discussed together in paragraph 10.2. Paragraph 10.3 evaluates how this translates to clinical practice, and paragraph 10.4 deals with the general limitations of our research. In addition, paragraph 10.5 and 10.6 glances into the future and discusses other fields of interest besides wheelchair technology. At the end of this chapter, paragraph 10.7 describes our final conclusions and answers the research questions (Q1-Q3) as stated above.

\subsection{Postural control and body load regulation}

To benefit the functional independence of the user, we discriminated between two forms of repositioning; i.e. physical repositioning and passive repositioning. The first refers to the users' physical ability to reposition body posture functionally. Chair configurations that involve a trade-off between posture stability and freedom of upper body movement might enhance the preserved ability to carry out some functional movement strategies without losing balance. Passive repositioning, on the other hand, refers to global chair modification that adjusts body posture in a passive manner. This means that the individual remains passive and undergoes postural variation as imposed by the chair. 


\subsubsection{Passive repositioning}

Able-bodied individuals do not suffer mobility problems since they shift sitting posture continuously. Because body posture is mainly determined by the alignment of the trunk, pelvis and thighs, proper postural control involves the ability to move all three body segments independently. Especially pelvis alignment plays a key role in the assessment of body posture and wheelchair configuration. Because the pelvis directly affects spinal curvature, postural stability and the forces that exert on the lower back and under the ischial buttock region, proper control of the pelvis is important. Until recently, no applications were known that could control the alignment of the pelvis separate from the trunk and thighs. Based on a parallelogram design that aligns the chair pivots with the anatomical axes for body segments rotation, we developed a technique that adjusts all three body segments independently. Together with simultaneous measurement of body segments orientation, this technique seemed applicable for proper control of body posture in sitting.

In previous chapters we demonstrated the effects of so-called body segments decoupling on postural alignment and body load in able-bodied and in individuals with a spinal cord injury (SCI). Based on experimental measurement of body segments rotation and angular chair modification, we developed a predictive model that relates chair configuration to body posture. Together with our findings about the effects on buttock load and low back load, knowledge has been obtained which could lead to an algorithm that controls body posture and regulates body load associated with wheelchair sitting discomfort. Because postural change was fully imposed from passive automatic chair modification, this type of repositioning is only preferred when the individual lacks the functionality to reposition him-/herself physically.

\subsubsection{Physical repositioning and functional movement}

Regarding the functional independence of wheelchair users, important criteria for new wheelchair design also involve interventions that provide postural stability without constraining functional movement. The ability to balance the trunk during unsupported body movement has therefore been analysed. In a search to find a trade-off between wheelchair configuration and freedom of upper body movement, we found that decoupled pelvis alignment significantly affects balance control in spinal cord injured individuals and that anterior tilted pelvis postures potentially benefit the functional performance in daily wheelchair use.

Functional balance during unsupported movement of the trunk and upper extremities relies on adequate neuromuscular proprioceptive responses of the central nervous system that keeps the body centre of gravity within specific stability limits. To increase the base of support and to bring the upper body centre of mass closer to the support surface, wheelchair-users who lack postural control often adopt a static kyphotic spinal posture with posterior tilted pelvis. Such postures allow large internal and external perturbation before reaching the boundaries of posture instability which makes that very little postural control is needed to maintain posturally stable. At the same time, very much effort is needed to change body posture for functional purpose and therefore a static sitting posture is often adopted.

In posterior tilted pelvis postures the lower spine adopts a kyphotic curvature 
and causes a passive extension torque that forces the trunk against the backrest. Where low SCI-individuals usually have enough muscle strength to counteract this passive resistance force, high SCI-individuals lack the strength that is needed to move the trunk in forward direction. When the pelvis rotates independently from the orientation of the trunk, the passive flexion-extension resistance of the lower spine changes together with its curvature. With this in mind, we ascribed the improvement of reaching distance in anterior tilted pelvis postures to a decrease in passive resistance against spinal flexion. However, when the pelvis rotates forward too far and the line of gravity comes close to the edges of the base of support, sitting balance is being jeopardised. Although very little effort is needed to change body posture functionally, the least external perturbation will cause postural instability.

To improve the functional movement range in individuals who lack the strength to counteract the force of gravity that keeps the trunk against the backrest, the stability area over which little effort is needed to change body posture functionally without jeopardising sitting balance must be enlarged. To maintain balance during perturbation, individuals with reduced or no trunk muscle function often use nonpostural shoulder muscles (e.g. Latisimus muscle, Trapezius muscle, Rhomboideus muscle, Serratus muscle etc.) to compensate for the stabilising loss of trunk muscles (Seelen and Vuurman, 1991, Seelen et al., 1998). Although the obtained stability is often limited, sometimes it can just be enough to carry out some functional movement without losing balance.

The armrest or a chair desk can provide an ideal base of support for the upper extremities to balance the trunk and enlarge the movement range in 'unstable' decoupled anterior tilted pelvis postures. This might also play an important role in wheelchair users who propel the wheels manually for their mobility. It has been reported in literature that postural alignment together with the preserved upper body functionality greatly influences the manual propulsion technique (Kulig et al., 2001, Mulroy et al., 2004, Minkel, 2000, van der Woude et al., 2001). To fully exploit the preserved shoulder muscle function and to propel the rim of the wheels more efficiently, sitting postures with a lordotic spinal curvature, anterior tilted stiff pelvis stabilisation and little resistance against forward trunk displacement might be beneficial. Chair configurations that involve anterior pelvis alignment due to forward backrest adjustment, and stiff pelvis stabilisation due to posterior seat inclination could therefore improve the efficiency of manual wheelchair propulsion which potentially decreases the overburden of shoulder muscles in daily wheelchair use.

\subsubsection{Additional local support surface manipulation}

Beside the potential benefit of both passive and physical repositioning on the regulation of body load associated with pressure ulcers and low back pain, additional manipulation of the support surface under the ischial buttock region seemed necessary to reduce the risk for pressure ulcers even more. This mainly counts for the buttock tissue under the ischial tuberosities. Because the contact forces in this region remain relatively high during so-called decoupled body segment adjustment, the tuberal support surface must be adjustable to periodically relieve the subtuberal buttock tissue and to prevent impairment of tissue perfusion in static sitting. Because it seemed that decoupled pelvis rotation and tuberal support adjustment 
are effective to alleviate the buttock tissue under the sacrum and tuberosities respectively, a combination of both techniques potentially benefits the viability of the internal buttock tissue within the entire ischial region.

\subsection{Clinical Application}

Throughout this thesis we demonstrated the effects of global chair modification and local seat support adaptation on postural alignment, body load and the performance of functional movement tasks in able-bodied and impaired subjects. To prevent all kinds of mobility problems, we strove to regulate body load from a combination of interventions. However, important questions arise about the sequence, frequency and extent each intervention must be applied for the best results per individual. Based on the principle of body segments decoupling and our findings with regard to the influence on body load and functional movement, the next section expounds our view on this challenging matter and discusses its value for clinical application.

\subsubsection{Adjustment to align body posture top-to-bottom}

For a good assessment of body posture and wheelchair configuration, the initial seating conditions must be adjusted with great care. It all starts with a good transfer to the wheelchair and proper alignment of body posture with the backrest, parallelogram and seat support. Our described system of body segments decoupling allows the possibility to align the head, trunk, pelvis and thighs one by one, based on the posture characteristics and functional ability of the user.

For an easy transfer to the chair, we must first lower the tuberal supports and tilt the whole seat mechanism forward. The individual is then seated with the ischial tuberosities (ITs) on top of the tuberal regions which are aligned with the axis for decoupled sagittal pelvis rotation. When the tuberal supports are lowered during transfer, it is easier to align body posture with chair configuration because the ITs sink in the seating surface and automatically positions the pelvis relative to the parallelogram mechanism. In our opinion, a 'correct' sitting posture must be imposed top-to-bottom, starting with a trunk angle which allows the head segment to be balanced with minimal effort required. Therefore, the backrest must be set in a way that the individual can look forward with minimal neck-muscle activation. According to Staarink this comes down to a backrest angle of approximately 115 degrees relative to the horizontal (Staarink, 2007).

Because the position of the footrests considerably affects the buttock interface pressure distribution, these must be adjusted with great care. When the footrests are set too low, the thighs function as a lever and decrease the pressure under the ITs. However, the contact load under the thighs at the edge of the seat support will increase significantly which could impair the function of superficial blood- and nerve vessels. On the other hand, when the footrests are set to high, the thighs are not supported by the seat and more weight exerts under the ischial buttock region. To distribute the interface pressure evenly on the seat support, the footrests must be set in a way that only the weight of the lower legs are supported and no joint forces act in the knees. This can be estimated from the gravitational force component on the seat if there are load-cells mounted under the support cushion. If not, some 
experience is needed to set the footrests by the use of visual inspection only.

When the back- and footrests are properly set, the next step is to align the pelvis and to adopt a 'neutral' curvature of the lower spine. This can be done by adjusting the configuration of the parallelogram. Because the alignment of the pelvis is decoupled from the seat support and backrest, pelvis angle can be set without affecting the orientation of the trunk and thighs. A desired lumbar spine curvature can therefore be imposed very precisely. It might even be possible to set the parallelogram configuration based on the forces that exert on the lower back which could be monitored when there are load-cells integrated under the seat support. Together with the predictive model from chapter 3 which relates angular chair configuration to sitting posture, the buttock support forces can be used to compute the net low back forces using static equations of equilibrium for the pelvis and thighs (chapters 7 and 8). This way, a 'correct' spinal posture with, for instance, minimal lumbar torque can be predicted and imposed due to proper adjustment of the parallelogram mechanism.

When the footrests, backrest and parallelogram configuration are carefully set, the next step is to adjust the angle of the seat support in a way that minimizes the shear force at the buttock-seat interface and reduces the risk to slide forward. Both criteria can be satisfied with minimal disturbance of the pelvis and trunk position. Because the seat pivots around an axis under the ITs, it is possible to control the orientation of the thighs, buttock shear and the risk to slide forward separate from the imposed spinal posture and load.

\subsubsection{Passive chair modification}

The configuration of the chair in which all body segments are individually aligned forms the basis from where chair modifications are needed for periodic relief of the load bearing tissue at the lower back and under the ischial buttock region. We must keep in mind that the frequency and extent of the imposed chair motion is highly dependent on the patient's physical ability. For individuals with reduced or no postural stability, the lack of functional body movement must be compensated by passive global chair modification and local support surface manipulation. The less upper body function is preserved, the more local surface manipulation is needed to regulate body load in a way that consistently prevents degeneration of body tissue. The same distinction can be made for the status of functional activity. When someone prefers to lean backwards and to adopt a relaxed body posture, more local support surface manipulation is needed than when a more active posture is preferred.

\subsubsection{1 'Active' and 'passive' pelvis postures}

In chapter 9 we demonstrated that postural stability could be influenced by adjusting the configuration of the parallelogram. It was found that configurations which involve a 'passive' posterior tilted pelvis posture allow large perturbation before reaching the boundaries of postural instability. Very little postural control is therefore needed to maintain stability, but at the same time, large effort is required to change body posture in a functional manner. In parallelogram configurations, which involve an 'active' anterior tilted pelvis posture, on the other hand, it is much easier to perform functional upper body movement. Unfortunately, the patient must be alert 
continuously since the smallest perturbation could already cause postural instability. Because it depends on the activity of that moment whether an active or passive pelvis posture is desired, it must be possible to adjust the parallelogram mechanism when demanded by the situation. Daily activities which are 'active' and require coordinated trunk and/or upper extremity movement (e.g. reaching tasks or manual wheelchair propulsion) benefit from an anterior tilted pelvis posture, and activities which are 'passive' and require no body movement (e.g. reading a book or watching television) benefit from a posterior tilted pelvis posture. For all activities in between, a pelvis posture which involves a 'neutral' spinal curvature might be most desirable.

\subsubsection{Periodic regulation of body load}

When someone adopts an active sitting posture and performs much functional movement, body load is regulated dynamically and less additional chair modification is needed to meet the criteria which consistently prevent tissue damage of load bearing structures. However, which type of modification is both beneficial and applicable in static wheelchair sitting? Chapters 4, 5 and 7 demonstrated in able-bodied individuals that sagittal and frontal decoupled pelvis rotations were effective to regulate the forces at the lower back and ischial buttock region. Because frontal pelvis rotations were imposed by changing the relative height between the left and right seat part, pressure relief at one side is always coupled to significant pressure increase at the opposite side. A technique that adjusts the ischial support surface rather than the orientation of the pelvis in frontal direction was therefore supposed to be better suited for periodic regulation of the subtuberal buttock tissue. As a consequence, we integrated two adjustable support elements under the ischial tuberosities to alleviate the subtuberal buttock tissue without affecting spinal posture and load. Based on simultaneous measurement of low back load, external buttock load and subtuberal blood supply, we suggest that a combination of sagittal decoupled pelvis rotation and tuberal support adjustment is most beneficial to regulate body load periodically which potentially prevent physical discomfort (e.g. low back pain and pressure ulcers) in prolonged static wheelchair sitting.

\subsubsection{Consequences for activities of daily life}

Another interesting advantage of these passive motion techniques is that the orientation of the trunk is not being disturbed. Body load can therefore be regulated with hardly any notice. However, some activities (e.g. reaching to or grabbing something of the shelf) require that the position of the trunk remains fixed relative to the surrounding. Although we expect that this would probably not be a big concern when body motion is induced in a very slow sinusoidal manner (e.g. $1 / 600 \mathrm{~Hz}$ ) and chair adaptation is undergone almost unconsciously, some activities (e.g. driving a car or sitting behind a table or office desk) demand that the trunk position does not change at all. For these situations, it might be better to move the seat support relative to a fixed backrest. However, it might be rather complex to design such an intervention especially when there are adjustable tuberal supports or even load-cells mounted in the seat. 


\subsubsection{Frequency and extent of chair modification}

Up to this point, we evaluated the influence of chair modification on body posture and body load, and we discussed what role each modification could play in the control of body posture and the prevention of pressure ulcers and low back pain. However, the unavoidable questions remain about the duration certain stress levels can be sustained and how often chair configuration must be adjusted to prevent tissue degeneration to take place.

The only thing we do know is that, in general, healthy individuals do not suffer from sitting related mobility problems since they shift body posture continuously. It might therefore be interesting to analyse 'healthy' sitting behaviour and learn about the frequency and extent able-bodied change their posture. Of course we must keep in mind that the physical status of i.e. the ischial buttock tissue in impaired persons is different than in able-bodied. Muscle atrophy and a poor peripheral perfusion of the load bearing tissue in this region make it questionable whether 'healthy' sitting could serve as a good basis for impaired persons as well. However, as a first indication, it might provide valuable information.

With the same purpose, 'healthy' sitting was recently evaluated by Linder-Ganz et al. (Linder-Ganz et al., 2007a). They reported that the frequency of postural change in healthy subjects was higher than recommended by the US Department of Health who advise wheelchair-users to shift body weight every 15 minutes (Agency for Health Care Policy and Research, 1994). Based on pressure changes under the ischial tuberosities, Linder-Ganz found that healthy subjects moved approximately every 6 minutes in sagittal direction and every 9 minutes in frontal direction. The extent of postural change which accompanied these pressure variations involved a moderate $15^{\circ}$ and $8^{\circ}$ for trunk and shoulder movements respectively. However, the evaluation of upper body movement alone might not provide a complete picture about the relation between postural change and the redistribution of buttock interface pressure. Because the pelvis supports the upper body and guides the gravitational forces component through the tuberosities to the seat, the position of the pelvis might be of even greater interest. When only focussing on upper body movements, significant pelvis motion might not be noticed and the observed effect of upper body posture on tuberal pressure could be overrated. Besides, it might even be possible that a large part of the observed upper body movement was performed to relieve stress in load bearing structures other than the ischial buttock tissue. For a better and more complete interpretation of sitting behaviour in healthy individuals, we believe that specific information about the position and orientation of the pelvis is essential. Nevertheless, it might still be rather difficult to generalize the frequency and extent of postural shifts in able-bodied because everyone changes body posture their own way. This is supported by the findings of Linder-Ganz et al. who reported a large variability between subjects.

Because postural shifts contribute to the regulation of stress levels in load bearing structures, additional measurement of body load might be of great interest. This way, relevant information can be obtained about the variation of body load which is needed to prevent that tissue degeneration takes place. This directly guides us to the most important limitations of our research. 


\subsection{Research Limitations}

To evaluate whether the simulator chair satisfied the functional design criteria and allows the regulation of body load associated with pressure ulcers and low back pain, experiments have been performed in which the external buttock load, subtuberal blood supply and the net low back forces were measured when subjected to alternating protocols of posture adjustment and local support surface manipulation. However, to quantify the risk for pressure ulcer onset, it has been recognized that the external buttock load provides only little information about the internal mechanical and physiological tissue conditions (Oomens et al., 2003). Therefore, we also measured the levels of buttock tissue oxygenation and perfusion which are considered in literature among the most important factors for quantitative assessment of peripheral perfusion and microcirculation in skin and subcutaneous tissue (Mathieu and Mani, 2007). Nonetheless, evidence from experimental research with muscle-cell cultures (Gawlitta et al., 2007, Gefen et al., 2008a, Gawlitta et al., 2008, Gefen et al., 2008b), finite element models (Linder-Ganz et al., 2007b, Brosh and Arcan, 2000, Ragan et al., 2002, Todd and Thacker, 1994, Lim et al., 2007, Makhsous et al., 2007), animals (Linder-Ganz et al., 2006, Stekelenburg et al., 2006) and humans (Ohura et al., 2007, Quintavalle et al., 2006) have demonstrated that the primary cause of deep tissue injury involves more than localised tissue ischemia only. This is supported by recent investigation of Stekelenburg at al. who showed that a complex interplay with prolonged excessive deformation potentially catalyses the irreversible process of tissue breakdown (Stekelenburg et al., 2008, Stekelenburg et al., 2007). Additional information about the internal deformation of ischial buttock tissue might therefore be necessary.

Linder-Ganz at al. developed a patient-specific real-time finite element model and reported that this new approach might be applicable to monitor stress/strain concentrations in deep muscle layers continuously (Linder-Ganz et al., 2005). Realtime finite element analysis with input from measurement of body posture and external buttock load might be of surplus value for the assessment of pressure ulcer risk and is therefore very interesting to evaluate in future experiments.

The same counts for the prediction of lumbar discomfort by just monitoring the net lumbosacral 'joint' forces. We are well aware that it is very hard to interpret and translate these findings directly to the onset of low back pain because we did not measure the mechanical and/or physiological spine conditions at inter-segmental level. Although we do think that our approach provided valuable information about the response of the lower spine during postural adjustment, finite element analysis are still needed for a better assessment of the internal spinal load associated with the feeling of discomfort at the lower back.

\subsection{Future Prospects}

The controllers of the simulator chair were designed to adjust body posture in a passive manner. As a consequence, no interaction was possible and postural change had to be undergone as imposed by the chair. To interact with the intention of the user and to benefit their functional independence, adaptive and compliant control behaviour is necessary. Well established methods to satisfy such behaviour can be 
obtained by impedance control or by springs that balance the trunk during upper body movement.

\subsubsection{Dynamic postural balance}

Based on the environmental dynamics and a relationship between the mass, stiffness and damping properties of the system, an impedance controller regulates the required interaction force to support a performed movement task as desired by the user. Such compliant control strategies are often used in exoskeletons that assist the functionality of the upper or lower extremities by reducing the muscle strength which is needed during movement.

Besides the support of arm or leg motion, impedance control might also be applicable to maintain postural stability during internal or external perturbation. With this purpose, Matjacic and Bajd designed a novel under-actuated control strategy for arm-free unsupported standing in paraplegic individuals (Matjacic and Bajd, 1998a, Matjacic and Bajd, 1998b). Based on theoretical and experimental evaluation, they demonstrated that paraplegics could use their preserved trunk muscles to balance the upper body in quite standing when the knees and hips were locked in extended positions and an artificially controlled stiffness of $8 \mathrm{Nm}$ /degree was implemented in the ankle joints. Such control strategy might also be applicable to control the orientation of the pelvis and to balance the upper body in paraplegic or even tetraplegic sitting. In combination with our introduced concept of decoupled trunk and pelvis rotation, impedance control of the parallelogram configuration (figure 10.1A) might allow paraplegics to use their preserved lumbothoracic muscles and physically adjust the alignment of the pelvis to change between spinal postures as shown in figure 10.1B. In complete tetraplegic individuals, on the other hand, no muscle activity is preserved in the lumbothoracic region. Therefore, the function of non-postural shoulder and arm muscles are required to control the orientation of the pelvis. Contrary to arm-free paraplegic sitting, one arm is always needed to maintain stability and to perform an upper body movement task as shown in figure 10.1C. A drawback of an under-actuated control strategy is that the individual must balance the upper body actively, which means that continuous coordinated muscle function is required. Even when sufficient sensory and motor abilities are preserved, functional activities which require an active posture for a longer period of time might be very fatiguing and for some paraplegics even impossible. Although this could be very suitable as a therapy and balance training in impaired sitting, control behaviour which adapts in a way that pelvis postures are always in static equilibrium might be better suited. In this way, there is no need to balance the upper body actively and all preserved motor function could be applied for the benefit of the performed functional trunk and upper extremity movement task.

\subsubsection{Static postural balance}

To minimize the effort to physically displace the upper body for functional purpose, the upper body centre of mass must be balanced perfectly. When the upper body is in static equilibrium throughout its whole range of movement, no external effort is theoretically needed to move the trunk and upper extremities quasi-statically. So- 

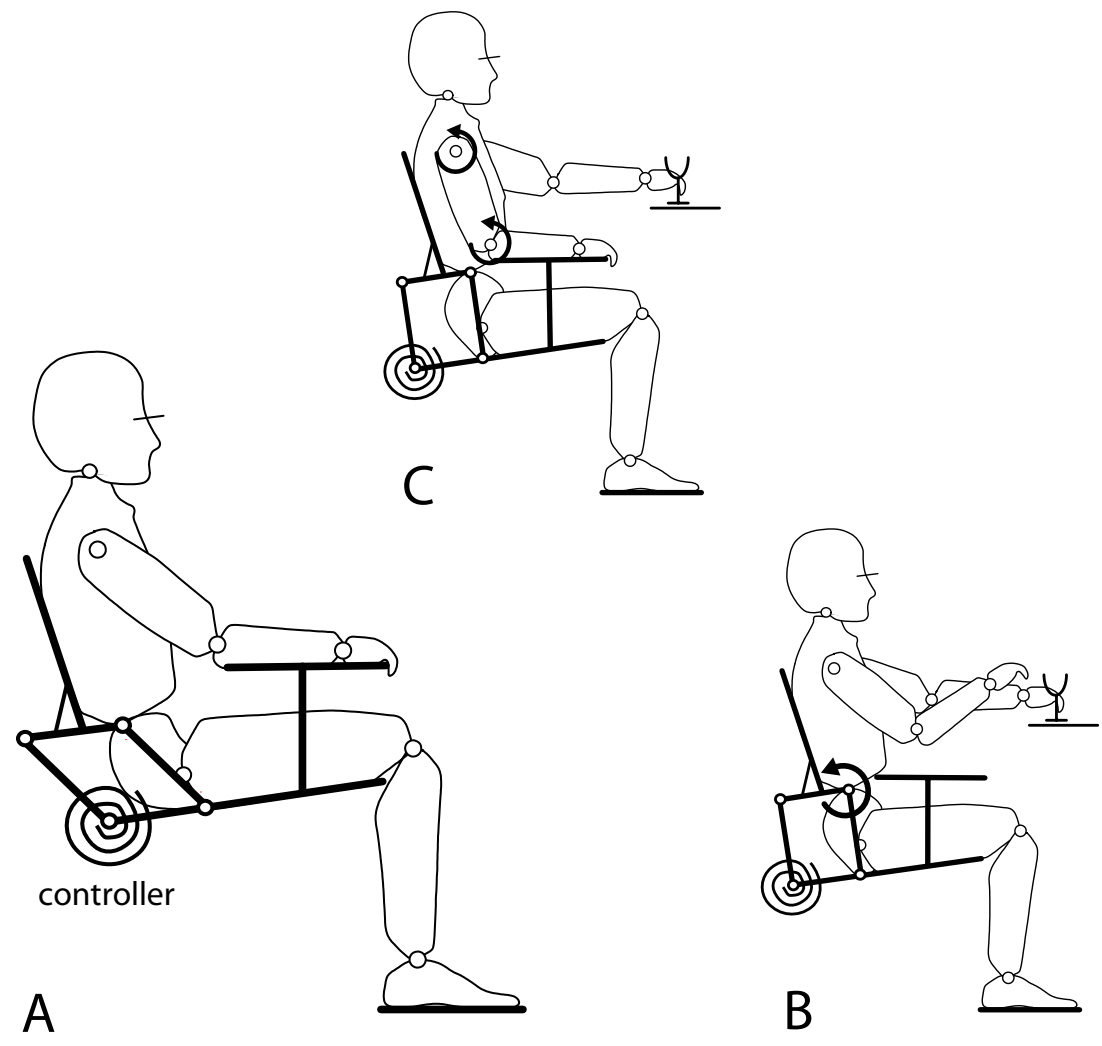

Figure 10.1: A: Dynamic seating system with parallelogram design and impedance or stiffness controller. B: Paraplegic sitting with lumbothoracic muscles to control the pelvis and balance the upper body during an arm-free movement task. C: Balanced tetraplegic sitting with the use of non-postural shoulder and arm muscles. One arm is always needed to maintain stability during upper body functional movement.

called energy free body movement requires constant potential energy and no internal friction what so ever. Because this means that the only demand for physical work is to initiate or to slow down the motion of the body, energy free systems that could balance the trunk during functional upper body movement might be of great interest for wheelchair users who lack the strength to maintain posturally stable during activities of daily life.

To create an energy free seating system, a counterweight might be used to compensate for the potential energy that that is needed to bring the upper body into balance. This technique was also applied to equilibrate the weight of the backrest in our simulator chair. Because the backrest was mounted on a sledge and a counterweight was used to compensate for its gravity potential, energy free vertical backrest movements were allowed. As a result, minimal friction occurs on the backrest interface because it moves along when the trunk tends to slide during sagittal postural adjustment. 
However, a major drawback of this technique is that an additional mass must be added to the system which is not desired in many clinical applications including (manual) wheelchair technology.

Energy free body motion can only be approximated since there is always some friction or viscosity which must be overcome. To reduce weight and to minimize friction during upper body movement, we rather use springs instead of a counter weight to create constant potential energy. Spring can compensates for the gravity potential of the upper body which is needed to change between posterior- and anterior tilted pelvis postures. When the upper body is statically balanced throughout the whole range of pelvis rotation, the only demand for physical effort is to begin or end a desired motion of the body. This allows wheelchair-users to control the pelvis in sagittal direction and helps them to change effortlessly between pelvis postures.

\subsection{Other fields of interest}

Besides the potential benefit for wheelchair-users who do not have the strength to reposition themselves physically, our described dynamic seating system might also be applicable in areas other than wheelchair technology. For instance, low back pain is also a big concern in able-bodied individuals who do sedentary work and sit in confined settings for hours. It has been reported that the passive flexion stiffness in moderate ranges of lumbar flexion already increases within two hours of static sitting (Beach et al., 2005). Some researchers (Beach et al., 2005, Parkinson et al., 2004) associated this with an increase in passive resistance of muscles at the lower back and suggested that these could be injured when full lumbar flexion tasks are performed after prolonged static sitting with flexed spinal posture. Therefore, the introduction of the backrest with parallelogram mechanism in sedentary occupations such as office-workers and car drivers might be applicable to induce sufficient lumbar motion and potentially reduce the risk for low back injury when sitting in confined settings for extended periods of time.

\subsection{Conclusions}

This paragraph looks back to our main research questions and answers them separately.

\section{Q1 How can sitting posture be controlled?}

Body posture is determined by the alignment of the trunk, pelvis and thighs and proper postural control involves the ability to move all three body segments independently. Especially pelvis alignment plays a key role in the assessment of body posture and wheelchair configuration. Based on a parallelogram design that aligns the chair pivots with the anatomical axes for body segments rotation, we developed a technique that adjusts all three body segments separately. Together with simultaneous measurement of body segments orientation, so-called body segments decoupling seemed applicable to control sitting posture when individuals lack the strength to do this themselves. 
Q2 What interventions are effective to regulate body load associated with physical discomfort (i.e. pressure ulcers and low back pain)?

Because the pelvis is supported by the seat and directly affects the curvature of the lumbar spine, the alignment of the pelvis plays an important role in the assessment of body load associated with pressure ulcers and lumbar discomfort. Our findings suggest that sagittal decoupled pelvis rotation is an effective technique that rotates the pelvis independent from the trunk and seat support, and alleviates the load bearing tissue under the sacrum and at the lower back. Because the pelvis roughly rotates around an axis under the ITs, the contact load in this region remains high. Adjustable support elements are needed for additional manipulation of the subtuberal buttock tissue. Therefore, a combination of both interventions seemed most suitable for simultaneous regulation of body load associated with physical discomfort in impaired sitting.

Q3 What interventions benefit the performance of functional movement in impaired sitting?

Because the pelvis forms the basis for trunk support and directly affects the curvature of the lumbar spine, it has been suggested in literature that seating interventions which adjust pelvis angle in sagittal direction might influence spinal posture and the performance of functional movement. We investigated this interesting manner in ten SCI-individuals by comparing the effects of parallelogram configuration on the ability to reach forward. Although the results between subjects were different, we concluded that decoupled pelvis alignment affects balance control in impaired sitting and that anterior tilted pelvis postures potentially benefit the functional performance in daily wheelchair-use. 


\section{References}


Ackery, A., Tator, C., Krassioukov, A. 2004. A global perspective on spinal cord injury epidemiology. Journal of Neurotrauma, 21 (10), 1355-70.

Agency for Health Care Policy and Research. 1994. Pressure ulcer treatment. Clinical Practice Guideline, no. 15, 1-25.

Aissaoui, R., Boucher, C., Bourbonnais, D., Lacoste, M., Dansereau, J. 2001a. Effect of seat cushion on dynamic stability in sitting during a reaching task in wheelchair users with paraplegia. Archives of Physical Medicine and Rehabilitation, 82 (2), 274-81.

Aissaoui, R., Lacoste, M., Dansereau, J. 2001b. Analysis of Sliding and Pressure Distribution During a Repositioning of Persons in a Simulator Chair. IEEE Transactions on Neural Systems and Rehabilitation Engineering, 9 (2), 215224.

Alm, M., Gutierrez, E., Hultling, C., Saraste, H. 2003. Clinical evaluation of seating in persons with complete thoracic spinal cord injury. Spinal Cord, 41 (10), 56371.

American Spinal Injury Association, 2000, International Standards for Neurologic Classification of SCI.

Aota, Y., Iizuka, H., Ishige, Y., Mochida, T., Yoshihisa, T., Uesugi, M., Saito, T. 2007. Effectiveness of a lumbar support continuous passive motion device in the prevention of low back pain during prolonged sitting. Spine, 32 (23), E674-7.

Argoubi, M., Shirazi-Adl, A. 1996. Poroelastic creep response analysis of a lumbar motion segment in compression. Journal of Biomechanics, 29 (10), 1331-9.

Bader, D. L. 1990. The recovery characteristics of soft tissues following repeated loading. Journal of Rehabilitation Research and Development, 27 141-50.

Beach, T. A., Mooney, S. K., Callaghan, J. P. 2003. The effects of a continuous passive motion device on myoelectric activity of the erector spinae during prolonged sitting at a computer workstation. Work, 20 (3), 237-44.

Beach, T. A., Parkinson, R. J., Stothart, J. P., Callaghan, J. P. 2005. Effects of prolonged sitting on the passive flexion stiffness of the in vivo lumbar spine. The Spine Journal, 5 (2), 145-54.

Beckert, S., Witte, M. B., Konigsrainer, A., Coerper, S. 2004. The impact of the MicroLightguide O2C for the quantification of tissue ischemia in diabetic foot ulcers. Diabetes Care, 27 (12), 2863-7.

Bell, A. L., Douglas, R. P., Brand, R. A. 1990. A Comparison Of The Accuracy Of Several Hip Center Location Prediction Methods. Journal of Biomechanics, 23 (6), 617 621.

Bidard, C., Rienstra, S., Veltink, P. H., Koopman, H. F. J. M., Grady, J., De Vries, J., Huttenhuis, L. 2000. Trunk stability while standing or sitting: a static analysis. 5 th Annual Conference of the International Functional Electrical Stimulation Society (IFESS), Aalborg.

Black, J., Baharestani, M., Cuddigan, J., Dorner, B., Edsberg, L., Langemo, D., Posthauer, M. E., Ratliff, C., Taler, G., Npuap. 2007. National Pressure Ulcer Advisory Panel's updated pressure ulcer staging system. Urologic Nursing, 27 (2), 144150.

Bouten, C. V., Oomens, C. W., Baaijens, F. P., Bader, D. L. 2003. The etiology of pressure ulcers: skin deep or muscle bound? Archives of Physical Medicine and Rehabilitation, 84 (4), 616-9. 
Brienza, D. M., Chung, K. C., Brubaker, C. E., Wang, J., Karg, T. E., Lin, C. T. 1996a. A system for the analysis of seat support surfaces using surface shape control and simultaneous measurement of applied pressures. IEEE Transactions on Rehabilitation Engineering, 4 (2), 103-13.

Brienza, D. M., Inigo, R. M., Chung, K. C., Brubaker, C. E. 1993. Seat support surface optimization using force feedback. IEEE Transactions on Biomedical Engineering, 40 (1), 95-104.

Brienza, D. M., Karg, P. E., Brubaker, C. E. 1996b. Seat cushion design for elderly wheelchair users based on minimization of soft tissue deformation using stiffness and pressure measurements. IEEE Transactions on Rehabilitation Engineering, 4 (4), 320-7.

Brosh, T., Arcan, M. 2000. Modeling the body/chair interaction - an integrative experimental-numerical approach. Clinical Biomechanics, 15 (3), 217-9.

Callaghan, J. P., Dunk, N. M. 2002. Examination of the flexion relaxation phenomenon in erector spinae muscles during short duration slumped sitting. Clinical Biomechanics, 17 353-360.

Campbell, F. M., Ashburn, A. M., Pickering, R. M., Burnett, M. 2001. Head and pelvic movements during a dynamic reaching task in sitting: implications for physical therapists. Archives of Physical Medicine and Rehabilitation, 82 (12), 165560.

Casey, V., Griffin, S., O'brien, S. B. 2001. An investigation of the hammocking effect associated with interface pressure measurements using pneumatic tourniquet cuffs. Medical Engineering and Physics, 23 (7), 511-7.

Chaffin, D., Woolley, C., Dickerson, C., Parkinson, M. 2004. Modeling of movement capability in the spinal cord injured population. International Journal of Industrial Ergonomics, 33 229-236.

Chen, C. L., Yeung, K. T., Bih, L. I., Wang, C. H., Chen, M. I., Chien, J. C. 2003. The relationship between sitting stability and functional performance in patients with paraplegia. Archives of Physical Medicine and Rehabilitation, 84 (9), 1276-81.

Coggrave, M. J., Rose, L. S. 2003. A specialist seating assessment clinic: changing pressure relief practice. Spinal Cord, 41 (12), 692-5.

Collins, F. 1999. Preventing pressure sores in the seated patient. Nursing Standard, 13 (42), 50-54.

Crane, B. A., Holm, M. B., Hobson, D., Cooper, R. A., Reed, M. P. 2007. A dynamic seating intervention for wheelchair seating discomfort. American Journal of Physical Medicine and Rehabilitation, 86 (12), 988-93.

Curtis, K. A., Kindlin, C. M., Reich, K. M., White, D. E. 1995. Functional reach in wheelchair users: the effects of trunk and lower extremity stabilization. Archives of Physical Medicine and Rehabilitation, 76 (4), 360-7.

Defloor, T., Schoonhoven, L. 2004. Inter-rater reliability of the EPUAP pressure ulcer classification system using photographs. Journal of Clinical Nursing, 13 (8), 952-9.

Defloor, T., Schoonhoven, L., Fletcher, J., Furtado, K., Heyman, H., Lubbers, M., Witherow, A., Bale, S., Bellingeri, A., Cherry, G., Clark, M., Colin, D., Dassen, T., Dealey, C., Gulacsi, L., Haalboom, J., Halfens, R., Hietanen, H., Lindholm, C., Moore, Z., Romanelli, M.,Soriano, J. V. 2005. Statement of the European 
Pressure Ulcer Advisory Panel--pressure ulcer classification: differentiation between pressure ulcers and moisture lesions. Journal of Wound, Ostomy and Continence Nursing, 32 (5), 302-6; discussion 306.

Delisle, A., Gagnon, M., Sicard, C. 1997. Effect of pelvic tilt on lumbar spine geometry. IEEE Transactions on Rehabilitation Engineering, 5 (4), 360-6.

Devivo, M. J. 1997. Causes and costs of spinal cord injury in the United States. Spinal Cord, 35 (12), 809-13.

Dickey, J. P., Gillespie, K. A. 2003. Representation of passive spinal element contributions to in vitro flexion-extension using a polynomial model: illustration using the porcine lumbar spine. Journal of Biomechanics, 36 (6), 883-8.

Dumas, R., Cheze, L., Verriest, J. P. 2007. Adjustments to McConville et al. and Young et al. body segment inertial parameters. Journal of Biomechanics, 40 (3), 54353.

Faiks, F. S., Reinecke, S. M., 1998. Investigation of spinal curvature while changing one's posture during sitting, Contemporary Ergonomics. pp: 113-117.

Ferguson, S. A., Marras, W. S. 1997. A literature review of low back disorder surveillance measures and risk factors. Clinical Biomechanics, 12 (4), 211-226.

Fujiwara, A., Lim, T. H., An, H. S., Tanaka, N., Jeon, C. H., Andersson, G. B., Haughton, V. M. 2000a. The effect of disc degeneration and facet joint osteoarthritis on the segmental flexibility of the lumbar spine. Spine, 25 (23), 3036-44.

Fujiwara, A., Tamai, K., An, H. S., Kurihashi, T., Lim, T. H., Yoshida, H., Saotome, K. 2000b. The relationship between disc degeneration, facet joint osteoarthritis, and stability of the degenerative lumbar spine. Journal of Spinal Disorders, 13 (5), 444-50.

Gawlitta, D., Boonen, K. J., Oomens, C. W., Baaijens, F. P., Bouten, C. V. 2008. The influence of serum-free culture conditions on skeletal muscle differentiation in a tissue-engineered model. Tissue Engineering Part A, 14 (1), 161-71.

Gawlitta, D., Oomens, C. W., Bader, D. L., Baaijens, F. P., Bouten, C. V. 2007. Temporal differences in the influence of ischemic factors and deformation on the metabolism of engineered skeletal muscle. Journal of Applied Physiology, 103 (2), 464-73.

Gefen, A., Cornelissen, L. H., Gawlitta, D., Bader, D. L., Oomens, C. W. 2008a. The free diffusion of macromolecules in tissue-engineered skeletal muscle subjected to large compression strains. Journal of Biomechanics, 41 (4), 845-53.

Gefen, A., Van Nierop, B., Bader, D. L., Oomens, C. W. 2008b. Strain-time cell-death threshold for skeletal muscle in a tissue-engineered model system for deep tissue injury. Journal of Biomechanics, 41 (9), 2003-12.

Gillespie, K. A., Dickey, J. P. 2004. Biomechanical role of lumbar spine ligaments in flexion and extension: determination using a parallel linkage robot and a porcine model. Spine, 29 (11), 1208-16.

Hastings, J. D., Fanucchi, E. R., Burns, S. P. 2003. Wheelchair configuration and postural alignment in persons with spinal cord injury. Archives of Physical Medicine and Rehabilitation, 84 (4), 528-34.

Hedman, T. P., Fernie, G. R. 1997. Mechanical response of the lumbar spine to seated postural loads. Spine, 22 (7), 734-43.

Henderson, J. L., Price, S. H., Brandstater, M. E., Mandac, B. R. 1994. Efficacy of three measures to relieve pressure in seated persons with spinal cord injury. Archives 
of Physical Medicine and Rehabilitation, 75 (5), 535-9.

Herder, J. L. 2001. Energy-Free Systems. Theory, conception and design of statically balanced spring mechanisms. Delft, The Netherlands, Delft University of Technology.

Hobson, D. A., Tooms, R. E. 1992. Seated lumbar/pelvic alignment. A comparison between spinal cord-injured and noninjured groups. Spine, 17 (3), 293-8.

Holm, S., Nachemson, A. 1983. Variations in the nutrition of the canine intervertebral disc induced by motion. Spine, 8 (8), 866-74.

Holzle, F., Loeffelbein, D. J., Nolte, D., Wolff, K. D. 2006. Free flap monitoring using simultaneous non-invasive laser Doppler flowmetry and tissue spectrophotometry. Journal of Cranio-Maxillofacial Surgery, 34 (1), 25-33.

Holzle, F., Swaid, S., Nolte, D., Wolff, K. D. 2003. Nutritive perfusion at donor site after microvascular fibula transfer. Microsurgery, 23 (4), 306-12.

Jackson, A. B., Dijkers, M., Devivo, M. J., Poczatek, R. B. 2004. A demographic profile of new traumatic spinal cord injuries: change and stability over 30 years. Archives of physical medicine and rehabilitation, 85 (11), 1740-8.

Janssen-Potten, Y. J., Seelen, H. A., Drukker, J., Huson, T., Drost, M. R. 2001. The effect of seat tilting on pelvic position, balance control, and compensatory postural muscle use in paraplegic subjects. Archives of Physical Medicine and Rehabilitation, 82 (10), 1393-402.

Janssen-Potten, Y. J., Seelen, H. A., Drukker, J., Reulen, J. P. 2000. Chair configuration and balance control in persons with spinal cord injury. Archives of Physical Medicine and Rehabilitation, 81 (4), 401-8.

Janssen-Potten, Y. J., Seelen, H. A., Drukker, J., Reulen, J. P., Drost, M. R. 1999. Postural muscle responses in the spinal cord injured persons during forward reaching. Ergonomics, 42 (9), 1200-15.

Janssen-Potten, Y. J., Seelen, H. A., Drukker, J., Spaans, F., Drost, M. R. 2002. The effect of footrests on sitting balance in paraplegic subjects. Archives of Physical Medicine and Rehabilitation, 83 (5), 642-8.

Keller, T. S., Spengler, D. M., Hansson, T. H. 1987. Mechanical behavior of the human lumbar spine. I. Creep analysis during static compressive loading. Journal of Orthopaedic Research, 5 (4), 467-78.

Kernozek, T. W., Lewin, J. E. 1998. Seat interface pressures of individuals with paraplegia: influence of dynamic wheelchair locomotion compared with static seated measurements. Archives of Physical Medicine and Rehabilitation, 79 (3), 313-6.

Kernozek, T. W., Wilder, P. A., Amundson, A., Hummer, J. 2002. The effects of body mass index on peak seat-interface pressure of institutionalized elderly. Archives of Physical Medicine and Rehabilitation, 83 (6), 868-71.

Koo, T. K., Mak, A. F., Lee, Y. L. 1996. Posture effect on seating interface biomechanics: comparison between two seating cushions. Archives of Physical Medicine and Rehabilitation, 77 (1), 40-7.

Kulig, K., Newsam, C. J., Mulroy, S. J., Rao, S., Gronley, J. K., Bontrager, E. L., Perry, J. 2001. The effect of level of spinal cord injury on shoulder joint kinetics during manual wheelchair propulsion. Clinical Biomechanics, 16 (9), 744-51.

Kwiatkowski, R. J., Inigo, R. M. 1993. A closed loop automated seating system. Journal of Rehabilitation Research and Development, 30 (4), 393-404. 
Lalonde, N. M., Dansereau, J., Aissaoui, R., Pauget, P., Cinquin, P. 2003. Differences between pelvic skin and bone landmark identification in different seated positions on spinal-cord injured subjects. IEEE Transactions on Biomedical Engineering, 50 (8), 958-66.

Lanzetta, D., Cattaneo, D., Pellegatta, D., Cardini, R. 2004. Trunk control in unstable sitting posture during functional activities in healthy subjects and patients with multiple sclerosis. Archives of Physical Medicine and Rehabilitation, 85 (2), 279-83.

Lazennec, J. Y., Charlot, N., Gorin, M., Roger, B., Arafati, N., Bissery, A., Saillant, G. 2004. Hip-spine relationship: a radio-anatomical study for optimization in acetabular cup positioning. Surgical and Radiologic Anatomy, 26 (2), 136-44.

Lengsfeld, M., Frank, A., Van Deursen, D. L., Griss, P. 2000a. Lumbar spine curvature during office chair sitting. Medical Engineering and Physics, 22 (9), 665-9.

Lengsfeld, M., Konig, I. R., Schmelter, J., Ziegler, A. 2007. Passive rotary dynamic sitting at the workplace by office-workers with lumbar pain: a randomized multicenter study. The Spine Journal, 7 (5), 531-40; discussion 540.

Lengsfeld, M., Van Deursen, D. L. Rohlmann, A., Van Deursen, L. L., Griss, P. 2000b. Spinal load changes during rotatory dynamic sitting. Clinical Biomechanics, 15 (4), 295-7.

Lim, D., Lin, F., Hendrix, R. W., Moran, B., Fasanati, C., Makhsous, M. 2007. Evaluation of a new sitting concept designed for prevention of pressure ulcer on the buttock using finite element analysis. Medical and Biological Engineering and Computing, 45 (11), 1079-84.

Lin, F., Parthasarathy, S., Taylor, S. J., Pucci, D., Hendrix, R. W., Makhsous, M. 2006. Effect of Different Sitting Postures on Lung Capacity, Expiratory Flow and Lumbar Lordosis. Archives of Physical Medicine and Rehabilitation, 87 504-9.

Linder-Ganz, E., Engelberg, S., Scheinowitz, M., Gefen, A. 2006. Pressure-time cell death threshold for albino rat skeletal muscles as related to pressure sore biomechanics. Journal of Biomechanics, 39 (14), 2725-32.

Linder-Ganz, E., Scheinowitz, M., Yizhar, Z., Margulies, S. S., Gefen, A. 2007a. How do normals move during prolonged wheelchair-sitting? Technology Health Care, 15 (3), 195-202.

Linder-Ganz, E., Shabshin, N., Itzchak, Y., Gefen, A. 2007b. Assessment of mechanical conditions in sub-dermal tissues during sitting: a combined experimental-MRI and finite element approach. Journal of Biomechanics, 40 (7), 1443-54.

Linder-Ganz, E., Shabshin, N., Itzchak, Y., Yizhar, Z., Siev-Ner, I., Gefen, A. 2008. Strains and stresses in sub-dermal tissues of the buttocks are greater in paraplegics than in healthy during sitting. Journal of Biomechanics, 41 (3), 567-80.

Linder-Ganz, E., Yarnitzky, G., Portnoy, S., Yizhar, Z., Gefen, A. 2005. Real-Time Finite Element Monitoring of Internal Stresses in the Buttock During Wheelchair Sitting to Prevent Pressure Sorces: Verification and Phantom Results. International Conference on Computational Bioengineering, Lisabon, Portugal.

Lis, A. M., Black, K. M., Korn, H., Nordin, M. 2007. Association between sitting and occupational LBP. European Spine Journal, 16 (2), 283-298.

Lucas, B., Asher, M., Mciff, T., Lark, R., Burton, D. 2005. Estimation of transverse plane pelvic rotation using a posterior-anterior radiograph. Spine, 30 (1), E20-7. 
Makhsous, M., Lim, D., Hendrix, R., Bankard, J., Rymer, W. Z., Lin, F. 2007a. Finite element analysis for evaluation of pressure ulcer on the buttock: development and validation. IEEE Transactions on Neural Systems and Rehabilitation Engineering, 15 (4), 517-25.

Makhsous, M., Lin, F., Hendrix, R. W., Hepler, M., Zhang, L. Q. 2003. Sitting with adjustable ischial and back supports: biomechanical changes. Spine, 28 (11), 1113-21; discussion 1121-2.

Makhsous, M., Priebe, M., Bankard, J., Rowles, D., Zeigler, M., Chen, D., Lin, F. 2007b. Measuring tissue perfusion during pressure relief maneuvers: insights into preventing pressure ulcers. The Journal of Spinal Cord Medicine, 30 (5), 497 507.

Makhsous, M., Rowles, D. M., Rymer, W. Z., Bankard, J., Nam, E. K., Chen, D., Lin, F. 2007c. Periodically relieving ischial sitting load to decrease the risk of pressure ulcers. Archives of Physical Medicine and Rehabilitation, 88 (7), 862-70.

Maltais, C., Dansereau, J., Aissaoui, R., Lacoste, M. 1999. Assessment of geometric and mechanical parameters in wheelchair seating: a variability study. IEEE Transactions on Rehabilitation Engineering, 7 (1), 91-8.

Mathieu, D., Mani, R. 2007. A review of the clinical significance of tissue hypoxia measurements in lower extremity wound management. The International Journal of Lower Extremity Wounds, 6 (4), 273-83.

Matjacic, Z., Bajd, T. 1998a. Arm-free paraplegic standing--Part I: Control model synthesis and simulation. IEEE Transactions on Rehabilitation Engineering, 6 (2), 125-38.

Matjacic, Z., Bajd, T. 1998b. Arm-free paraplegic standing--Part II: Experimental results. IEEE Transactions on Rehabilitation Engineering, 6 (2), 139-50.

Maurer, C. L., Sprigle, S. 2004. Effect of seat inclination on seated pressures of individuals with spinal cord injury. Physical Therapy, 84 (3), 255-61.

Mcgregor, A. H., Mccarthy, I. D., Dore, C. J., Hughes, S. P. 1997. Quantitative assessment of the motion of the lumbar spine in the low back pain population and the effect of different spinal pathologies of this motion. European Spine Journal, 6 (5), 308-15.

Meriam, J. L., Kraige, L. G. 1993 Engineering Mechanics: Statics, John Wiley \& Sons, Inc.

Minkel, J. L. 2000. Seating and mobility considerations for people with spinal cord injury. Physical Therapy, 80 (7), 701-9.

Moes, C. C. 1998. Measuring the tilt of the pelvis. Ergonomics, 41 (12), 1821-1831.

Mork, J. P., Westgaard, R. H. 2009. Back posture and low back muscle activity in female computer workers: A field study. Clinical Biomechanics, 24 (169-175),

Mulroy, S. J., Farrokhi, S., Newsam, C. J., Perry, J. 2004. Effects of spinal cord injury level on the activity of shoulder muscles during wheelchair propulsion: an electromyographic study. Archives of Physical Medicine and Rehabilitation, 85 (6), 925-34.

O’sullivan, P. B., Dankaerts, W., Burnett, A. F., Farrell, G. T., Jefford, E., Naylor, C. S., O'sullivan, K. J. 2006. Effect of different upright sitting postures on spinalpelvic curvature and trunk muscle activation in a pain-free population. Spine, 31 (19), E707-12.

Ohura, T., Ohura, N., Oka, H. 2007. Incidence and Clinical Symptoms of Hourglass 
and Sandwich-shaped Tissue Necrosis in Stage IV Pressure Ulcers. Wounds, 19 (11), 310-319.

Oomens, C. W., Bressers, O. F., Bosboom, E. M., Bouten, C. V., Blader, D. L. 2003. Can loaded interface characteristics influence strain distributions in muscle adjacent to bony prominences? Computer Methods in Biomechanics and Biomedical Engineering, 6 (3), 171-80.

Parkinson, R. J., Beach, T. A., Callaghan, J. P. 2004. The time-varying response of the in vivo lumbar spine to dynamic repetitive flexion. Clinical Biomechanics, 19 (4), 330-6.

Peirce, S. M., Skalak, T. C., Rodeheaver, G. T. 2000. Ischemia-reperfusion injury in chronic pressure ulcer formation: a skin model in the rat. Wound Repair and Regeneration, 8 68-76.

Peleg, S., Dar, G., Medlej, B., Steinberg, N., Masharawi, Y., Latimer, B., Jellema, L., Peled, N., Arensburg, B., Hershkovitz, I. 2007a. Orientation of the human sacrum: anthropological perspectives and methodological approaches. American Journal of Physical Anthropology, 133 (3), 967-77.

Peleg, S., Dar, G., Steinberg, N., Peled, N., Hershkovitz, I., Masharawi, Y. 2007b. Sacral orientation revisited. Spine, 32 (15), E397-404.

Post, M. W., De Witte, L. P., Van Asbeck, F. W., Van Dijk, A. J., Schrijvers, A. J. 1998. Predictors of health status and life satisfaction in spinal cord injury. Archives of physical medicine and rehabilitation, 79 (4), 395-401.

Powell, E. S., Pyburn, R. E., Hill, E., Smith, K. S., Ribbands, M. S., Mickelborough, J., Pomeroy, V. M. 2002. Measurement of sitting balance using the Manchester Active Position Seat (MAPS): a feasibility study. Clinical Rehabilitation, 16 (6), 661-8.

Pozzo, T., Levik, Y., Berthoz, A. 1995. Head and trunk movements in the frontal plane during complex dynamic equilibrium tasks in humans. Experimental Brain Research, 106 (2), 327-38.

Quintavalle, P. R., Lyder, C. H., Mertz, P. J., Phillips-Jones, C., Dyson, M. 2006. Use of high-resolution, high-frequency diagnostic ultrasound to investigate the pathogenesis of pressure ulcer development. Advances in Skin and Wound Care, 19 (9), 498-505.

Ragan, R., Kernozek, T. W., Bidar, M., Matheson, J. W. 2002. Seat-interface pressures on various thicknesses of foam wheelchair cushions: a finite modeling approach. Archives of Physical Medicine and Rehabilitation, 83 (6), 872-5.

Rajbhandari, S. M., Harris, N. D., Tesfaye, S., Ward, J. D. 1999. Early identification of diabetic foot ulcers that may require intervention using the micro lightguide spectrophotometer. Diabetes Care, 22 (8), 1292-5.

Reenalda, J., Van Geffen, P., Jannink, M., Nederhand, M., IJzerman, M., Rietman, H. 2009. Effects of actuated tuberal support on sitting load, tissue perfusion and tissue oxygenation. Submitted for publication.

Reinecke, S. M., Hazard, R. G., Coleman, K. 1994. Continuous passive motion in seating: a new strategy against low back pain. Journal of Spinal Disorders, 7 (1), 2935.

Reswick, J., Rogers, J., 1976. Experience at Rancho Los Amigos Hospital with devices and techniques to prevent pressure sores., in: Kennedy, R. M., Cowden, J. M. \& Scales, J. T. (Eds.) Bedsore Biomechanics. McMillan, London, pp. 301-10. 
Samuelsson, K., Larsson, H., Thyberg, M., Tropp, H. 1996. Back Pain and Spinal Deformity-Common Among Wheelchair Users with Spinal Cord Injuries. Scandinavian Journal of Occupational Therapy, 3 (1), 28-32.

Seelen, H. A., Janssen-Potten, Y. J., Adam, J. J. 2001. Motor preparation in postural control in seated spinal cord injured people. Ergonomics, 44 (4), 457-72.

Seelen, H. A., Potten, Y. J., Drukker, J., Reulen, J. P., Pons, C. 1998. Development of new muscle synergies in postural control in spinal cord injured subjects. Journal of Electromyography and Kinesiology, 8 (1), 23-34.

Seelen, H. A., Potten, Y. J., Huson, A., Spaans, F., Reulen, J. P. 1997. Impaired balance control in paraplegic subjects. Journal of Electromyography and Kinesiology, 7 (2), 149-160.

Seelen, H. A., Vuurman, E. F. 1991. Compensatory muscle activity for sitting posture during upper extremity task performance in paraplegic persons. Scandinavian Journal of Rehabilitation Medicine, 23 (2), 89-96.

Sprigle, S., Dunlop, W., Press, L. 2003a. Reliability of bench tests of interface pressure. Assistive Technology, 15 (1), 49-57.

Sprigle, S., Flinn, N., Wootten, M.,Mccorry, S. 2003b. Development and testing of a pelvic goniometer designed to measure pelvic tilt and hip flexion. Clinical Biomechanics, 18 (5), 462-5.

Sprigle, S., Maurer, C., Prysock, A. 2007. Load redistribution in standing, tilt-in-space, and reclining wheelchairs. In: International Seating Symposium.

Sprigle, S., Wootten, M., Bresler, M., Flinn, N. 2002. Development of a noninvasive measure of pelvic and hip angles in seated posture. Archives of Physical Medicine and Rehabilitation, 83 (11), 1597-602.

Sprigle, S., Wootten, M., Sawacha, Z., Theilman, G. 2003c. Relationships among cushion type, backrest height, seated posture, and reach of wheelchair users with spinal cord injury. Journal of Spinal Cord Medicine, 26 (3), 236-43.

Staarink, H. A. M. (1995) Sitting posture, comfort and pressure. Faculty of Industrial Design Engineering. Delft, Delft University of Technology.

Staarink, H. A. M. 2007 Zo zit het! Van Gorcum.

Stekelenburg, A., Gawlitta, D., Bader, D. L., Oomens, C. W. 2008. Deep tissue injury: how deep is our understanding? Archives of Physical Medicine and Rehabilitation, 89 (7), 1410-3.

Stekelenburg, A., Oomens, C. W., Strijkers, G. J., De Graaf, L., Bader, D. L., Nicolay, K. 2006. A new MR-compatible loading device to study in vivo muscle damage development in rats due to compressive loading. Medical Engineering and Physics, 28 (4), 331-8.

Stekelenburg, A., Strijkers, G. J., Parusel, H., Bader, D. L., Nicolay, K., Oomens, C. W. 2007. Role of ischemia and deformation in the onset of compression-induced deep tissue injury: MRI-based studies in a rat model. Journal of Applied Physiology, 102 (5), 2002-11.

Stinson, M., Porter, A., Eakin, P. 2002. Measuring interface pressure: a laboratory based investigation into the effects of repositioning and sitting. American Journal of Occupational Therapy, 56 (2), 185-190.

Stockton, L., Parker, D. 2002. Pressure relief behaviour and the prevention of pressure ulcers in wheelchair users in the community. Journal of Tissue Viability, 12 (3), 84, 88-90, 92 passim. 
Stockton, L., Rithalia, S. 2007. Is dynamic seating a modality worth considering in the prevention of pressure ulcers? Journal of Tissue Viability, 17 15-21.

Swain, I. D., Bader, D. L. 2002. The measurement of interface pressure and its role in soft tissue breakdown. Journal of Tissue Viability, 12 (4), 132-4, 136-7, 140-6.

Tam, E. W., Mak, A. F., Lam, W. N., Evans, J. H., Chow, Y. Y. 2003. Pelvic movement and interface pressure distribution during manual wheelchair propulsion. Archives of Physical Medicine and Rehabilitation, 84 (10), 1466-72.

Todd, A. I., Bennett, A. I., Christie, C. J. 2007. Physical implications of prolonged sitting in a confined posture - A literature review. Ergonomics SA, 19 (2), 7-21.

Todd, B. A., Thacker, J. G. 1994. Three-dimensional computer model of the human buttocks, in vivo. Journal of Rehabilitation Research and Development, 31 (2), 111-9.

Tsuji, S., Ichioka, S., Sekiya, N., Nakatsuka, T. 2005. Analysis of ischemia-reperfusion injury in a microcirculatory model of pressure ulcers. Wound Repair and Regeneration, 13 209-215.

Urban, J. P., Smith, S., Fairbank, J. C. 2004. Nutrition of the intervertebral disc. Spine, 29 (23), 2700-9.

Van der Woude, L. H., Veeger, H. E., Dallmeijer, A. J., Janssen, T. W., Rozendaal, L. A. 2001. Biomechanics and physiology in active manual wheelchair propulsion. Medical Engineering and Physics, 23 (10), 713-33.

Van Deursen, D. L., Lengsfeld, M., Snijders, C. J., Evers, J. J., Goossens, R. H. 2000. Mechanical effects of continuous passive motion on the lumbar spine in seating. Journal of Biomechanics, 33 (6), 695-9.

Van Deursen, L. L., Patijn, J., Durinck, J. R., Brouwer, R., Van Erven-Sommers, J. R., Vortman, B. J. 1999. Sitting and low back pain: the positive effect of rotary dynamic stimuli during prolonged sitting. European Spine Journal, 8 (3), 187 93.

Van Dieen, J. H., De Looze, M. P., Hermans, V. 2001. Effects of dynamic office chairs on trunk kinematics, trunk extensor EMG and spinal shrinkage. Ergonomics, 44 (7), 739-50.

Van Geffen, P., Molier, B. I., Reenalda, J., Veltink, P. H., Koopman, H. F. J. M. 2008a. Body segments decoupling in sitting: Control of body posture from automatic chair adjustments. Journal of Biomechanics, 41 (16), 3419-3425.

Van Geffen, P., Reenalda, J. 2008. DYNASIT: An Assistive Seating System that Controls Sitting Posture and Regulates Body Load Associated with Sitting Related Mobility Problems. In: RESNA 2008, Washington, USA.

Van Geffen, P., Reenalda, J., Veltink, P. H., Koopman, H. F. J. M. 2008b. Effects of sagittal postural adjustments on seat reaction load. Journal of Biomechanics, 41 (10), 2237-45.

Van Geffen, P., Reenalda, J., Veltink, P. H., Koopman, H. F. J. M. 2009. Decoupled pelvis rotation in sitting: A passive motion technique that regulates buttock load associated with pressure ulcer development. Journal of Biomechanics (in press).

Verver, M. M., Van Hoof, J., Oomens, C. W., Van De Wouw, N., Wismans, J. S. 2003. Estimation of spinal loading in vertical vibrations by numerical simulation. Clinical Biomechanics, 18 (9), 800-11. 
Viswanathana, M., Jorgensena, M. J., Kittusamy, N. K. 2006. Field evaluation of a continuous passive lumbar motion system among operators of earthmoving equipment. International Journal of Industrial Ergonomics, 36, 651-659.

Whittemore, R. 1998. Pressure-reduction support surfaces: a review of the literature. Journal of Wound, Ostomy and Continence Nursing, 25 (1), 6-25.

Williams, M. M., Hawley, J. A., Mckenzie, R. A., Van Wijmen, P. M. 1991. A comparison of the effects of two sitting postures on back and referred pain. Spine, 16 (10), 1185-91.

Wyndaele, M., Wyndaele, J. J. 2006. Incidence, prevalence and epidemiology of spinal cord injury: what learns a worldwide literature survey? Spinal Cord, 44 (9), 523-9. 


\section{Appendix A}

\section{Design Process}

Based on: Paul van Geffen \& Jasper Reenalda, Award nominated design paper in RESNA 2008, Washington DC, USA, June 26-30 


\section{Introduction}

This appendix gives an impression about the design process of the simulator chair and provides a short description about the most important features. For an extensive and detailed evaluation regarding the function of the prototype, we refer to the chapters of this thesis.

\section{Functional requirements}

\section{Decoupled body segments control}

Able-bodied individuals do not suffer mobility problems since they continuously shift body posture. Because body posture is mainly determined by the alignment of the trunk, pelvis and thighs, proper posture control involves the ability to move all three body segments independently. However, for individuals who cannot functionally reposition themselves, no applications are known that allows decoupled trunk, pelvis and thighs adjustments.

A 'classical' chair with one pivot between the seat and backrest makes it impossible to adjust the trunk, pelvis and thighs independently and introduces sliding when changing its configuration (Fig A.1A). A concept with the axes of rotation aligned within the lumbar region and under the ischial tuberosities allows independent body segments adjustment in the sagittal plane (Fig. A.1B). Based on a parallelogram from which the backrest is actuated, a mechanical concept for decoupled body segments adjustment is shown in figure A.1C. Actuating the configuration of the parallelogram rotates the pelvis in the sagittal plane. The whole mechanism rotates around a pivot under the tuberosities and is externally actuated. In the frontal plane, actuating the relative height between the left and right seat part rotates the pelvis sideways around an axis between the ischial tuberosities. For lateral trunk rotation, the backrest is actuated around a pivot within the lumbothoracic region.

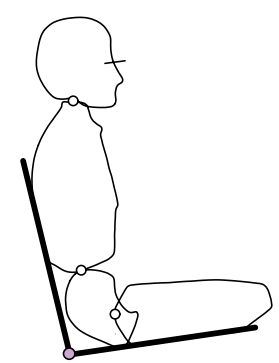

A

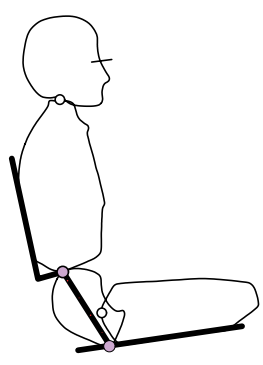

B

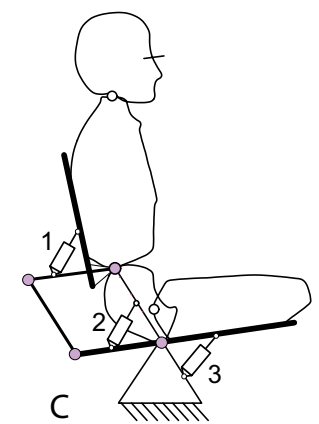

C

Figure A.1: Principle for sagittal postural adjustments. A: Classical chairs with one pivot between the seat and backrest do not allow independent trunk, pelvis and thighs adjustments. B: Concept with two axes of rotation aligned within the lumbar region and under the ischial tuberosities enables independent body segments control in the sagittal plane. C: Based on a parallelogram, a mechanical concept for decoupled body segments adjustment is shown. The backrest is adjusted from the parallelogram (1), the parallelogram from the back seat (2) and the seat from the under frame (3). 


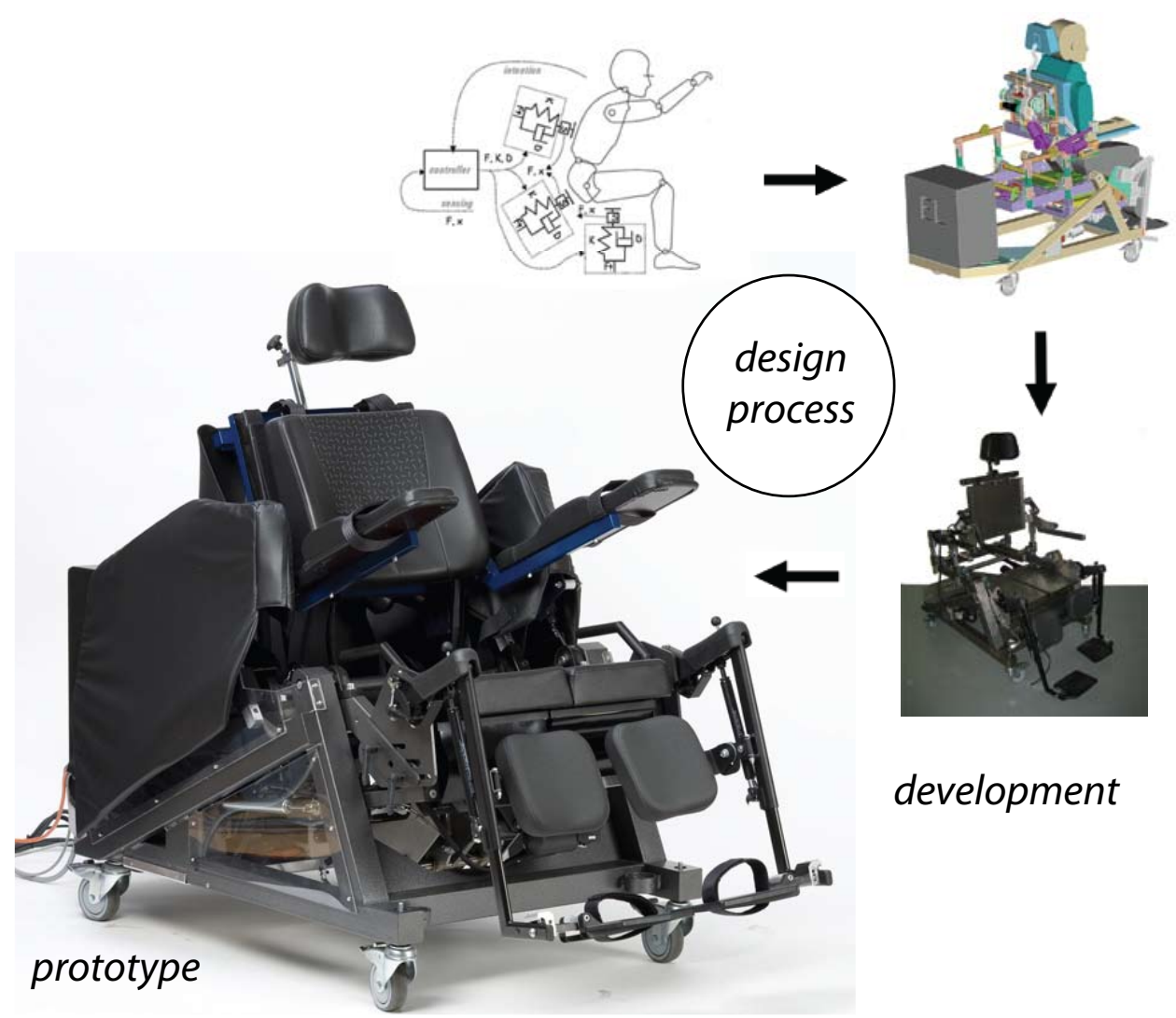

Figure A.2: Design process of the simulator chair. In close collaboration with our group, the simulator chair was designed and built by the engineering company Demcon BV (Oldenzaal, the Netherlands).

\section{Ischial support surface manipulation}

The soft tissues underneath the ischial tuberosities are most vulnerable for sustained tissue deformation when sitting in a static posture. Besides the positive influence of decoupled body segments control on the redistribution of buttock load, two adjustable tuberal supports must be integrated in the seating surface for additional manipulation of the ischial buttock tissue.

\section{Prototype}

Based on these requirements, a prototype of the simulator chair was developed as shown in figure A.2. 
The fully adjustable simulator chair is computer-aided (Matlab/Simulink ${ }^{\circledR}$ environment) and instrumented with the following features:

1. A concept that decouples the alignment of the trunk, pelvis and thighs is integrated to control body posture when individuals lack the strength to do this themselves (Fig. A.3).

2. Built-in measurement for real-time feedback of chair configuration and body posture.

3. A sledge mounted backrest to minimize friction at the trunk/backrest interface. Frictionless vertical trunk translations are allowed to align the axes for body segments rotation exactly with the axes for chair adjustment.

4. Two adjustable force sensing support elements under the ischial tuberosities. Aligned with the seat pivot and spaced according to an average inter-tuberal distance of $120 \mathrm{~mm}$, two adjustable force sensing support elements are integrated under the seat support. Both support elements are independently adjustable and designed to relieve pressure under the tuberosities only (Fig A.4).

5. A force sensing seating plane. For accurate 3D measurements of seat reaction load, six uni-axial load cells are integrated in the seating surface.

Furthermore, all mechanical and electrical mechanisms are carefully covered and protected to ensure full safety for the subject and bystanders. 


\section{sagittal}

\section{frontal}

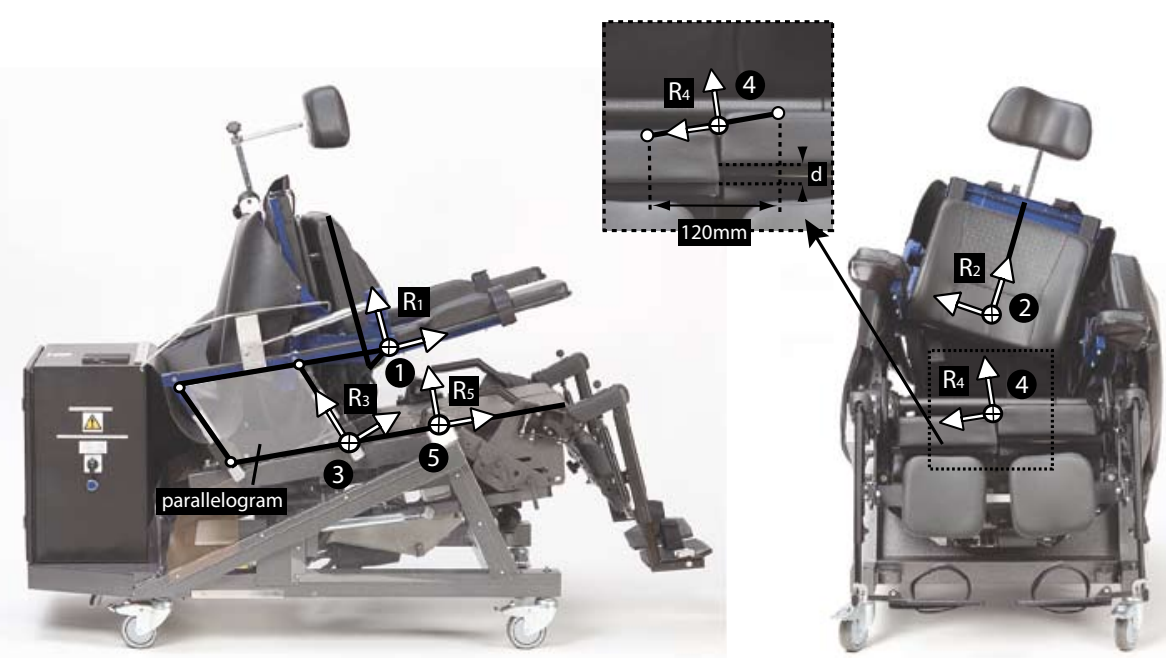

Figure A.3: Sagittal and frontal view of the computer-aided adjustable simulator chair instrumented with the concepts for decoupled body segments adjustment. The axes of rotation (1-5) together with the local reference frames $\left(R_{1}-R_{5}\right)$ for all chair segments adjustments are shown. $R_{1}, R_{3}$ and $R_{5}$ define the frames for the backrest, parallelogram and seat in the sagittal plane. $R_{2}$ and $R_{4}$ define the frames for the backrest and seat in the frontal plane. $R_{4}$ is constructed from the height $\mathrm{d}[\mathrm{mm}]$ between the left and right seat part assuming an average ischial tuberal width of $120 \mathrm{~mm}$.
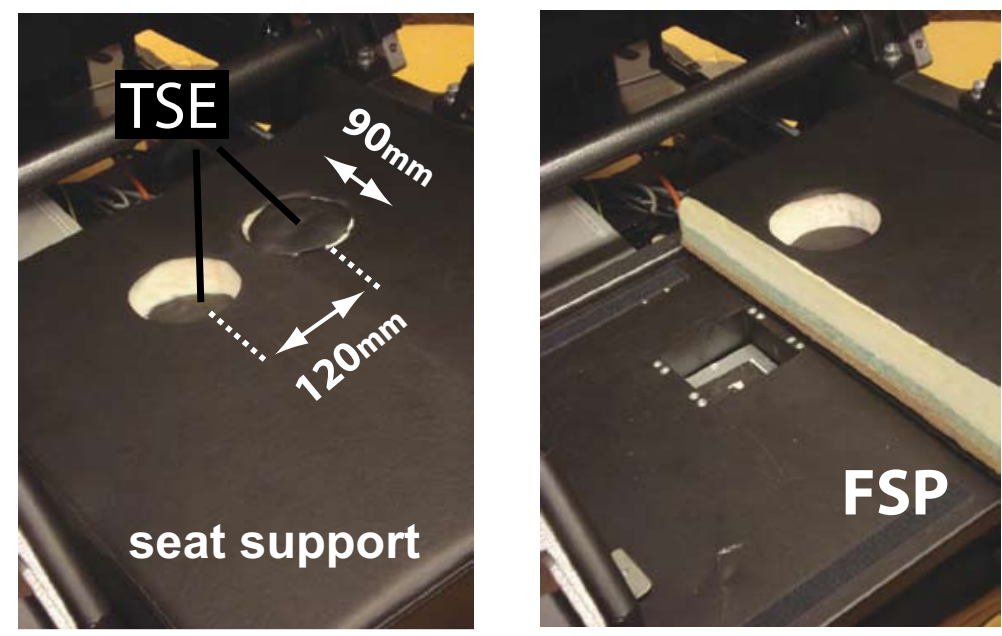

Figure A.4: A seat support with force-sensing under plate (FSP) and two round (Ø 90mm) adjustable force-sensing tuberal support elements (TSE) spaced according to an inter-tuberal distance of $120 \mathrm{~mm}$. 


\section{Appendix B}

\section{Research Consortium}




\section{Introdution}

This appendix introduces the partners of the research consortium and gives a short description about their role in the project.

\section{Partners}

Funded by the Dutch Ministry of Economical Affairs (SenterNovem, Grant TSIT3043), the DYNSIT-consortium was established to develop an 'intelligent' chair for individuals with reduced postural stability to enhance functional movement and to prevent physical discomfort in prolonged static sitting. The following partners participated in this consortium:

- Institute of Biomedical Technology (BMTI), University of Twente.

- $\quad$ Roessingh Research and Development (RRD)

- Demcon BV

- Welzorg Nederland BV

- PRsella BV

From the Institute of Biomedical Technology, the research was performed by Paul van Geffen and supervised by Peter Veltink (PhD) and Bart Koopman (PhD). From the Roessingh Research and Development, the research was performed by Jasper Reenalda and supervised by Michiel Jannink (PhD), Marc Nederhand (MD, PhD), Hans Rietman (MD, PhD) and Maarten IJzerman (PhD). In close collaboration with Paul van Geffen from BMTi and Jasper Reenalda from RRD, the engineering company Demcon BV designed and built the experimental simulator chair. Bert Faber from Welzorg Nederland BV, and Louis Huttenhuis from PRsella BV participated in this consortium for their expertise in seating ergonomics.

\section{Institute of Biomedical Technology (BMTI)}

BMTI is an interdepartmental institute of the University of Twente, focussing on research and education in biomedical technology. It is recognised world-wide as a leading institute in this multi-disciplinary field of science where engineering concepts and methods are employed to solve and understand medical and biological problems. BMTI is a strong determinant for the profile of the university and its strategic partners. Depending on the problem, BMTI's research may focus on various issues from (early) diagnostics to medical intervention, and more rehabilitation related activities. Dissemination of results is an integral part of the research and training activities of the institute. To this end, BMTI is actively involved in (the formation of) various business-oriented networks to support scouting and coaching of potential spinout companies and stimulate new business development (source: www.bmti. utwente.nl). 


\section{Roessingh Research and Development (RRD)}

Roessingh Research and Development (RRD), the largest Dutch scientific research centre in rehabilitation technology, is an independent R\&D organisation linked to The Roessingh rehabilitation centre in Enschede. Its mission is to generate innovations in rehabilitation care through top class academic research. RRD is internationally recognised as a centre of excellence in the fields of restoration of human function, non-invasive neuromuscular assessment and pain rehabilitation. Since 2004, RRD and care experts from The Roessingh have headed the National Innovation Centre for Pain Rehabilitation and Rehabilitation Technology, which is certified by the Dutch Ministry of Health, Welfare and Sport. RRD has a strong cooperation with the Twente University where 2 scientific chairs are filled in by RRD/Roessingh researchers: Neuromuscular Control and Rehabilitation Medicine and Technology (source: www. rrd.nl).

\section{DEMCON}

DEMCON is an engineering company from Oldenzaal, the Netherlands, and realizes high-tech mechatronic systems and products through research, development and production. With its mechatronic approach to design, DEMCON generates high-end solutions for complex issues in various markets, ranging from semicon and defence to medical and life sciences. DEMCON has all the required disciplines at its disposal to create complete mechatronic solutions - from mechanics, electronics and software to industrial design, prototyping and production. By integrating all these disciplines and focusing on manageability, DEMCON knows how to bring complex projects to a successful conclusion (source: www.demcon.nl).

\section{Welzorg Nederland}

Welzorg Nederland BV is a nation wide producer of home care products and ergonomical appliances. The company is a leader in its market and provides mobility aids for elderly and handicapped throughout the Netherlands. Their costumers include municipalities, healthcare institutions, health insurance companies, and endusers. It sells its products through a network of outlets (source: www.welzorg.nl).

\section{PRsella}

In the conviction that sitting in a wheelchair could greatly be improved, PRsella has been established by Louis Huttenhuis. PRsella is a company in Oldenzaal, the Netherlands, that works in close collaboration with researchers, healthcare organizations (doctors and therapists), end-users, technicians and software developers. PRsella has been involved for many years in research and the development of products that make an optimal seating quality possible (source: www.pr-sella.nl). 
Appendix C

List of Abbreviations 


\section{Abbreviations}

In this appendix, a list of frequently used abbreviations is included for the convenience of the reader. We expect that this facilitates the reading process and improves the interpretation of our findings.

\section{Abbreviation}

(L,R)ASIS

(L,R)HJC

$(\mathrm{L}, \mathrm{R}) \mathrm{KJC}$

$(\mathrm{L}, \mathrm{R}) \mathrm{LFE}$

(L,R)MFE

(L,R)PSIS

$(\mathrm{L}, \mathrm{R}) \mathrm{SIS}$

3D

AIS

ANOVA

ASIA

BMI

BS

C7

CJC

DI

DPR

FS

FSP

ITs

LB

LBP

LJC

$\mathrm{O} 2 \mathrm{C}$

$\mathrm{PM}$

PMD

PU

PX

SCI

TA

TSA

TSE

UB

US

\section{Definition}

(Left, Right) Anterior Superior Iliac Spine

(Left, Right) Hip Joint Centre

(Left, Right) Knee Joint Centre

(Left, Right) Lateral Femoral Epicondyles

(Left, Right) Medial Femoral Epicondyles

(Left, Right) Posterior Superior Iliac Spine

(Left, Right) midpoint between (L,R)PSIS and (L,R)ASIS

Three Dimensional

ASIA Impairment Scale

ANalysis Of Variance

American Spinal Cord Injury Association

Body Mass Index

Back Seat

$7^{\text {th }}$ Cervical vertebra

Cervical Joint Centre

Dispersion Index

Decoupled Pelvis Rotation

Front Seat

Force-sensing Seating Plane

Ischial Tuberosities

Lower Back Support

Low Back Pain

Lumbar Joint Centre

Oxygen-2-See ${ }^{\circ}$

Pelvis Mold

Pressure Mapping Device

Pressure Ulcers

Processus Xypoid

Spinal Cord Injury

Tuberal Area

Tuberal Support Adjustment

Tuberal Support Elements

Upper Back Support

Upper Sternum 


\section{Summary}


Wheelchair-users with reduced postural stability have often problems with the performance of functional movement tasks during activities of daily life. Functional balance during unsupported movement of the trunk and upper extremities relies on adequate neuromuscular proprioceptive responses of the central nervous system that keeps the body centre of mass within specific stability limits. To increase the base of support and to bring the body centre of mass closer to the support surface, a static kyphotic spinal posture with posterior tilted pelvis is often adopted.

Prolonged static sitting unconditionally leads to all kinds of physical discomfort including the formation of pressure ulcers, low back injury, lumbar immobility and joint stiffness. It has been recognised that most mobility problems occur from sustained mechanical tissue loading and that dynamic seating interventions are needed when individuals do not have the strength to reposition themselves physically.

The main target group of this research involved all wheelchair-users who suffer from mobility problems due to prolonged static sitting. Because physical discomfort is also a concern in able-bodied persons who do sedentary work (i.e. office-works, vehicle driving) and sit still in confined setting for hours, this group also received attention. However, we mainly focussed on individuals with a spinal cord injury (SCI) because of the devastating impact on the patient's functional, medical, financial and psychosocial well-being and its significant costs to society.

In this thesis we developed an experimental simulator chair and we evaluated several seating interventions that are designed to enhance functional movement and to prevent physical discomfort due to prolonged static sitting. The most important functional requirements involved the possibility to control body posture and to regulate body load associated with pressure ulcers and low back pain. Theoretical and experimental evaluations were performed in able-bodied subjects and in subjects with a spinal cord injury (SCI) and the following research questions have been answered:

Q1 How can sitting posture be controlled?

Q2 What interventions are effective to regulate body load associated with physical discomfort (i.e. pressure ulcers and low back pain)?

Q3 What interventions benefit the performance of functional movement in impaired sitting?

\section{Postural control}

Literature revealed that the alignment of the pelvis plays a key role in the assessment of body posture and wheelchair configuration in relation to wheelchair seating discomfort. Because pelvis angle directly affects spinal curvature, postural stability and the forces that exert on the lower back and under ischial buttock region, control of the pelvis is of great importance.

To monitor pelvis angle for proper pelvis control, chapter 2 explored whether it is feasible to predict pelvis angle from seat support forces based on a theoretical analysis and measurements for experimental validation. Under the experimented seating conditions, no body postures were found in which pelvis angle could directly be estimated from the forces on the seat. This indicated that pelvis angle could not 
be controlled and that an alternative method had to be found.

We therefore instrumented the simulator chair with an intervention that decouples the trunk, pelvis and thighs by aligning the chair pivots with the anatomical axes for body segments rotation. Based on experimental measurement of body posture and chair segments modification in healthy individuals, in chapter 3 we developed a predictive model that seemed applicable to control sitting posture from automatic adjustment of the angular configuration of chair.

\section{Regulation of Body Load}

Now we demonstrated the influence of so-called decoupled body segments adjustment on sitting posture, the next step was to evaluate whether the simulator chair is also applicable to regulate body load associated with pressure ulcer development and low back discomfort.

Chapters 4, 5 and 7 demonstrated in able-bodied individuals that a combination of seat inclination and decoupled pelvis rotations were effective to regulate the forces at the lower back and ischial buttock region simultaneously.

Because frontal pelvis rotations were imposed by changing the relative height between the left and right seat part, pressure relief at one side was always coupled to significant pressure increase at the opposite side. Two adjustable support elements were therefore integrated under the ischial tuberosities. Positive effects of this dynamic tuberal support on the redistribution of ischial buttock load and pattern of blood supply have been demonstrated in chapter 6 .

\section{Clinical Evaluation}

For clinical practice, the effects of local and global chair adjustment on body load were also evaluated in individuals who cannot functionally reposition themselves. Impairments to the neuromuscular function influence postural response (e.g. muscle spasms) from chair modification and made clinical investigation necessary. Chapter 8 therefore investigated the beneficial effects the dynamic tuberal support in combination with decoupled pelvis rotation in ten male SCI-subjects. Based on simultaneous measurement of low back load, external buttock load and subtuberal blood supply, it was concluded that a combination of both interventions is most beneficial to regulate body load periodically and potentially prevent physical discomfort (e.g. low back pain and pressure ulcers) in prolonged static wheelchair sitting.

Besides the potential benefits for body load, important criteria for new wheelchair design also involve interventions that provide posture stability without constraining functional movement. The ability to balance the trunk during unsupported body movement has therefore been analysed in chapter 9. It was found that decoupled pelvis alignment affected balance control in impaired sitting and that anterior tilted pelvis postures potentially benefit the functional performance in daily wheelchairuse.

\section{Discussion and Conclusions}

At the end of this thesis, chapter 10 provided a general discussion about our main findings and their clinical implications. It looked back to our objectives and evaluated 
future prospects that are necessary to bring our obtained knowledge into clinical practice.

In conclusion, this thesis gave answer to the initial research questions as summarised below.

\section{Q1 How can sitting posture be controlled?}

Body posture is determined by the alignment of the trunk, pelvis and thighs and proper postural control involves the ability to move all three body segments independently. Especially pelvis alignment plays a key role in the assessment of body posture and wheelchair configuration. Based on a parallelogram design that aligns the chair pivots with the anatomical axes for body segments rotation, we developed a technique that adjusts all three body segments separately. Together with simultaneous measurement of body segments orientation, so-called body segments decoupling seemed applicable to control sitting posture when individuals lack the strength to do this themselves.

Q2 What interventions are effective to regulate body load associated with physical discomfort (i.e. pressure ulcers and low back pain)?

Because the pelvis is supported by the seat and directly affects the curvature of the lumbar spine, the alignment of the pelvis plays an important role in the assessment of body load associated with pressure ulcers and lumbar discomfort. Our findings suggest that sagittal decoupled pelvis rotation is an effective technique that rotates the pelvis independent from the trunk and seat support, and alleviates the load bearing tissue under the sacrum and at the lower back. Because the pelvis roughly rotates around an axis under the ITs, the contact load in this region remains high. Adjustable support elements are needed for additional manipulation of the subtuberal buttock tissue. Therefore, a combination of both interventions seemed most suitable for simultaneous regulation of body load associated with physical discomfort in impaired sitting.

\section{Q3 What interventions benefit the performance of functional movement in impaired sitting?}

Because the pelvis forms the basis for trunk support and directly affects the curvature of the lumbar spine, it has been suggested in literature that seating interventions which adjust pelvis angle in sagittal direction might influence spinal posture and the performance of functional movement. We investigated this interesting manner in ten SCI-individuals by comparing the effects of parallelogram configuration on the ability to reach forward. Although the results between subjects were different, we concluded that decoupled pelvis alignment affects balance control in impaired sitting and that anterior tilted pelvis postures potentially benefit the functional performance in daily wheelchair-use. 


\section{Samenvatting}


Rolstoelgebruikers met een beperkte rompstabiliteit hebben vaak problemen met het uitvoeren van dagelijkse bewegingstaken. Het handhaven van de stabiliteit bij bewegingen waarbij het lichaam niet wordt ondersteund, is veelal afhankelijk van een goede neuromusculaire proprioceptieve feedback van het centrale zenuwstelsel. Deze zorgt er namelijk voor dat het lichaamszwaartepunt binnen bepaalde stabiliteitsgrenzen blijft, en dat een bewegingstaak zonder veel problemen kan worden uitgevoerd. Om toch stabiel te kunnen blijven wordt dan ook vaak een kyfotische zithouding met een achterovergekanteld bekken aangenomen. Een dergelijke houding is erg stabiel maar leidt tevens tot zeer statisch zitgedrag. Langdurig zitten in deze houding leidt ontegenzeggelijk tot fysieke problemen zoals decubitus, lage rugklachten, immobiliteit van de wervelkolom en gewrichtsstijfheid.

Veelal wordt aangenomen dat de meeste van deze problemen ontstaan door aanhoudende belasting van het weefsel. Dynamische stoelinterventies zijn daarom noodzakelijk om periodiek het weefsel te ontlasten als de gebruiker dit niet zelfstandig kan.

Hoewel dit onderzoek zich voornamelijk richtte op rolstoelgebruikers die niet zelfstandig kunnen verzitten, keken we tevens naar de fysieke ongemakken die optreden bij 'gezonde' personen die zittend werk doen en waarbij de arbeidsomstandigheden het niet toelaten om regelmatig van houding te veranderen. Desalniettemin verdienden dwarslaesiepatiënten het meeste van onze aandacht vanwege de enorme functionele, medische, financiële en psychologische impact en bijkomstige kosten voor de maatschappij.

Binnen het Dynasit project is een experimentele zitopstelling ontwikkeld waarmee verschillende stoelinterventies zijn geëvalueerd met als doel om de functionele bewegingsvrijheid te vergroten en om fysieke ongemakken tegen te gaan. De belangrijkste eigenschappen waaraan de opstelling moest voldoen betroffen de mogelijkheid om de zithouding te controleren en gelijkertijd de lichaamsbelasting te reguleren. Hierdoor kan mogelijk het risico op decubitus en lage rugklachten worden verminderd.

Theoretische en experimentele evaluaties zijn uitgevoerd met 'gezonde' personen en met dwarslaesie patiënten. Aan de hand hiervan zijn de volgende onderzoeksvragen beantwoord:

Q1 Hoe kan de zithouding worden gecontroleerd?

Q2 Welke interventies zijn effectief om de lichaamsbelasting te reguleren zodat er geen fysieke ongemakken (bv. decubitus en lage rugklachten) ontstaan?

Q3 Welke interventies verbeteren de functionele bewegingsmogelijkheid bij rolstoelgebruikers met een beperkte rompstabiliteit?

\section{Houdingscontrole}

Uit de literatuur is gebleken dat de stand van het bekken een belangrijke rol speelt bij het beoordelen van de zithouding en stoelconfiguratie in relatie tot het optreden van fysieke ongemakken. Omdat het bekken direct is gekoppeld aan de curvatuur van de wervelkolom, de houdingsstabiliteit, en de belasting in de rug en onder het zitvlak, is het enorm belangrijk om de stand hiervan op de juiste manier te controleren. 
Op basis van simulaties en experimentele metingen hebben we in hoofdstuk 2 onderzocht of het mogelijk is om de stand van het bekken te voorspellen aan de hand van de krachten op de zitting. Onder de experimentele zitcondities zijn echter geen zithoudingen gevonden waarbij de stand van het bekken direct kan worden afgeleid van de krachten op de zitting. Hieruit bleek dat het niet mogelijk is om op deze manier het bekken te controleren en dat naar een alternatieve methode moest worden gezocht.

De experimentele zitopstelling is vervolgens uitgerust met een interventie die de stand van de romp, het bekken en de bovenbenen ontkoppelt door de draaipunten van de stoel gelijk te leggen met de anatomische draaipunten van het lichaam. In hoofdstuk 3 zijn experimenten uitgevoerd waarbij de zithouding en stoelconfiguratie simultaan zijn gemeten bij gezonde personen. Op basis hiervan is een voorspellend model ontwikkeld waarmee de zithouding kan worden gecontroleerd door de stoelconfiguratie automatisch te veranderen.

\section{Reguleren van lichaamsbelasting}

$\mathrm{Nu}$ duidelijk was wat de effecten zijn van deze techniek op het veranderen van de zithouding, richtten we ons vervolgens op de mogelijkheid om de zitbelasting zodanig te reguleren dat decubitus en lage rugklachten niet op zullen treden.

De hoofdstukken 4, 5 en 7 tonen aan dat bij gezonde personen een combinatie van zittingkanteling en ontkoppelde bekkenrotatie effectief is om gelijktijdig de belasting in de onderrug en onder het ischiale deel van het zitvlak te reguleren. Omdat frontale bekkenrotaties werden opgelegd door de relatieve hoogte tussen het linker en rechter deel van de zitting te variëren, was een afname van contactdruk aan de ene kant altijd gekoppeld aan een toename van druk aan de andere kant.

Om dit te voorkomen waren twee verstelbare ondersteuningselementen geïntegreerd in de zitting onder de zitbeenderen (tubera). Beide elementen konden onafhankelijk van elkaar worden aangestuurd. De effecten op de zitbelasting en doorbloeding zijn vervolgens onderzocht in hoofdstuk 6. Positieve effecten zijn gevonden van deze dynamische tubera-ondersteuning op de verdeling van de ischiale zitdruk en het patroon van de doorbloeding in huid en onderliggend (spier)weefsel.

\section{Klinische evaluatie}

Ten behoeve van de klinische toepasbaarheid hebben we tevens onderzocht wat de effecten van locale en globale stoelaanpassingen zijn bij rolstoelgebruikers die niet zelfstandig kunnen verzitten. Een verstoorde neuromusculaire functie beïnvloedt namelijk de manier waarop het lichaam reageert op dergelijke interventies. Door de mogelijke invloed van bijvoorbeeld spierspasmen is het dan ook belangrijk om klinische experimenten uit te voeren.

In hoofdstuk 8 onderzochten we de voordelige effecten van de dynamische tuberaondersteuning en ontkoppelde bekkenrotatie bij 10 mannelijke dwarslaesiepatiënten. Op basis van experimenten waarbij de belasting in de onderrug, de belasting onder het zitvlak en de sub-tuberale doorbloeding simultaan zijn gemeten bleek een combinatie van interventies het meest geschikt om de lichaamsbelasting te reguleren en mogelijk fysieke problemen zoals decubitus en rugklachten te voorkomen.

Bij het ontwerpen van rolstoelen is het tevens erg belangrijk om een goede 
zitstabiliteit te garanderen zonder hiermee de bewegingsvrijheid te beperken. De mate waarin iemand met een verminderde rompstabiliteit kan balanceren tijdens het uitvoeren van een reikbeweging zonder dat het lichaam wordt ondersteund, is geanalyseerd in hoofdstuk 9. Hieruit concludeerden we dat de zogenoemde ontkoppelde bekkenkanteling de zitbalans beïnvloedt en dat een voorwaarts gekantelde bekkenstand mogelijk de functionele bewegingsvrijheid vergroot tijdens het uitvoeren van dagelijkse handelingen.

\section{Algemene discussie en conclusies}

In de algemene discussie bespreken we onze bevindingen en hun implicaties in klinische zin. Hoofdstuk 10 blikt terug op onze vraagstellingen en evalueert hoe de ontwikkelde kennis kan worden ingezet voor klinische doeleinden.

Concluderend kunnen we stellen dat dit proefschrift antwoord geeft op de onderzoeksvragen die hieronder nogmaals kort worden samengevat.

\section{Q1 Hoe kan de zithouding worden gecontroleerd?}

De zithouding wordt bepaald door de stand van de romp, het bekken en de bovenbenen. Een goede houdingscontrole betreft dan ook de mogelijkheid om alle drie de lichaamssegmenten onafhankelijk te bewegen. Vooral de stand van het bekken speelt hierbij een belangrijke rol. Op basis van een parallellogramconcept dat de draaipunten van de stoel gelijk legt aan de anatomische draaipunten van het lichaam is een techniek ontwikkeld waarmee de standen van de romp, bekken en bovenbenen onafhankelijk kunnen worden versteld. Door gelijktijdig de stoelconfiguratie te monitoren kan deze nieuw ontwikkelde techniek mogelijk worden ingezet om de zithouding te controleren bij rolstoelgebruikers die dit niet zelfstandig kunnen.

Welke interventies zijn effectief om de lichaamsbelasting te reguleren zodat er geen fysieke ongemakken (bv. decubitus en lage rugklachten) ontstaan?

Het bekken wordt ondersteund door de zitting en heeft een grote invloed op de curvatuur van de lumbale wervelkolom. De stand van het bekken speelt dan ook een belangrijke rol bij het beoordelen van de lichaamsbelasting in relatie tot decubitus en lage rugklachten. Uit ons onderzoek is gebleken dat de ontkoppelde bekkenrotatie in het sagittale vlak een effectieve methode is om het weefsel onder het sacrum en in de onderrug periodiek te ontlasten. Omdat het bekken grotendeels draait om een as onder de tubera, blijft de belasting in deze regio relatief hoog. Verstelbare ondersteuningselementen onder de tubera zijn dan ook noodzakelijk voor additionele manipulatie van het sub-tuberale weefsel. Een combinatie van interventies bleek het meest geschikt voor het reguleren van de zitbelasting en het voorkomen van fysiek ongemak bij rolstoelgebruikers.

Q3 Welke interventies verbeteren de functionele bewegingsmogelijkheid bij rolstoelgebruikers met een beperkte rompstabiliteit?

Het bekken is de basis voor rompondersteuning en heeft tevens invloed op de curvatuur van de lumbale wervelkolom. Uit de literatuur bleek dan ook dat stoelinterventies die 
het bekken in sagittale richting roteren mogelijk de functionele bewegingsvrijheid van rolstoelgebruikers met een beperkte rompstabiliteit kunnen beïnvloeden. We hebben dit onderzocht bij 10 mannelijke dwarslaesiepatiënten door de effecten van verschillende parallellogramconfiguraties tijdens een voorwaartse reikbeweging te vergelijken. Ondanks grote inter-individuele verschillen concludeerden we dat de zogenoemde ontkoppelde bekkenkanteling invloed heeft op de zitbalans en mogelijk de functionele bewegingsvrijheid tijdens dagelijkse activiteiten ten goede komt. 
Dankwoord 


\section{LUCTOR .........}

Hoewel je het uiteindelijk toch helemaal zelf moet doen, zijn er velen die ik enorm dankbaar ben voor hun steun in morele, begeleidende, financiële, opbeurende, collegiale en vriendschappelijke zin van het woord. Ik heb veel aan jullie te danken, dus mag het best een lang verhaal worden.

Ik kan het me nog goed herinneren: Barcelona, december 2004. Twee hoog op een balkonnetje in de Spaanse zon. Een telefonisch groepsgesprek met ene dr.ir. H.F.J.M Koopman en ene prof.dr.ir. P.H. Veltink was de eerste stap naar datgene waar ik vier jaar lang hard aan heb moeten werken, namelijk: één lettertje minder.

Bart, als promotor en dagelijks begeleider stond je aan de basis van het proefschrift dat je nu in handen hebt. Onze werkbesprekingen waren voor mij als kersverse bewegingswetenschapper in het begin niet altijd even makkelijk. Met speels gemak rolde de rotatiematrices en overdrachtsfuncties uit je pen die zich vervolgens bij mij een weg naar boven worstelden. Eenmaal terug op mijn kamer moest ik alles vaak nog eens goed op een rijtje zetten en voor mezelf herhalen wat je zojuist aan me duidelijk probeerde te maken. Ik kan me geen moment herinneren dat je niet de tijd hebt genomen om mijn soms vicieuze gedachtegang te doorbreken. Vaak wachtte je geduldig af totdat je wist waar ik nou eigenlijk mee worstelde. Aan een half woord had je soms genoeg om me weer op het juiste spoor te zeten. Door jouw manier van begeleiden leerde ik zelfstandig te werken, vroegtijdig valkuilen te omzeilen en lastige vraagstellingen op een logische manier te beredeneren. Bart, ik heb de afgelopen vier jaar veel van je geleerd en ben je hier erg dankbaar voor.

Peter, als medepromotor ben ook jij erg belangrijk geweest tijdens mijn promotietraject. Eerst in de Hogekamp en daarna om de hoek in de Zuidhorst. Jij hebt mij op een ongekend gedreven en enthousiaste manier de kneepjes van het vak geleerd. Hoe druk je ook was, je hebt elke letter van mij gelezen. Het leidde tot een mooie wisselwerking: hoe meer ik produceerde, hoe kritischer jouw commentaar. Dan was het tijd om passen op de plaats te maken en terug te keren naar de essentie van mijn verhaal. Wanneer ik je zocht, kon ik je altijd makkelijk traceren. Met hoogfrequente pas(s) snelde je door de gang van afspraak naar afspraak. Met de juiste filter had ik je dan vaak snel te pakken. Peter, ik kijk terug op een leuke en leerzame samenwerking die ik niet snel zal vergeten.

Jasper, wat hebben we ontzettend intensief samengewerkt. Als klankbord, lotgenoot, sparringpartner, rechterhand, collega-onderzoeker, criticus, co-autheur, typical example, healthy subject, filmacteur, fotomodel, Reenalda et. al., personal trainer en geducht koffiepartner hebben we de afgelopen vier jaar vele uren in het Dynasit project gestoken. In tegenstelling tot mijzelf, wist je al snel wat je te doen stond. Jij keek dan ook vaak met argusogen naar de zoveelste bachelor- of masteropdracht waarmee ik op de proppen kwam. Velen hiervan stierven dan ook een vroege dood. Tja, je bedenkt soms de vreemdste dingen als je 4 uur per dag reist. Jasper, ik wens je heel veel succes met de laatste loodjes en ik hoop je in de toekomst zowel persoonlijk als professioneel nog vaak tegen te komen. Bedankt dat je mijn paranimf wilt zijn!

Ik kijk met enorm veel plezier terug op de afgelopen vier jaar bij de vakgroep. Martin en Jan S, hierbij reken ik jullie natuurlijk ook! Zaalvoetballen, hardlopen, squashen, 
congressen, mountainbiken en biertjes drinken op het terras of in de kroeg heb ik voor geen goud willen missen. Jammer dat die laatste trein altijd al om half elf richting Amersfoort vertrok. Of toch half twaalf? Herman, het was maar goed dat Arno zijn mobiel op tijd opnam, anders zou het een duur nachtje zijn geworden. Het LOPES team en Arno, vooral bij jullie kwam ik in de beginfase regelmatig voor wat hulp. Ralf, als doorgewinterde matlab-goeroe was je nooit te beroerd om me duidelijk te maken hoe het wel moest. Ik hoop niet dat je eigen project hier te veel onder heeft geleden :-p. Jan, als nuchtere Fries heb je een onuitwisbare indruk op me gemaakt. Het was vaak gezellig tot in de late uurtjes. Ons uitje in Amersfoort zal ik dan ook niet snel vergeten. Of toch wel?! Edwin, als collega-bewegingswetenschapper heb je de lat erg hoog gelegd bij de vakgroep. Nog bedankt daarvoor ;-). Zonder jouw dondermail weet ik niet of alles op tijd af zou zijn. We zullen het ook nooit weten. Itskes, dezelfde naam, maar o-zo verschillend. Jullie zijn een aanwinst voor de vakgroep en ik vind het jammer dat jullie pas zo laat de UT hebben gevonden. Jasper, Dorien, Edsko, Jeroen, Bart V-en, Alfred, Nico, Ramazan and Frans, als vaste en minder vaste collega's was het altijd erg gezellig in de wandelgangen en tijdens de koffiepauzes. Sorry dat ik hier soms maar kort aan deel nam. Dorien, voor een gastcollege ergonomie sta ik natuurlijk nog altijd open. Gert Jan, Geert en Wouter, jullie goede ondersteuning maakt(e) elk project tot een succes. Nikolai, ze zeggen wel eens dat een goede netwerk- en systeembeheerder niet opvalt. In positieve zin geldt dat zeker niet voor jou. Jij was nooit te beroerd om mij te ondersteunen in het VR-lab en mij te helpen met mijn veelal zelf gecreëerde computerproblemen. Gerdine en Martijn W, een goede buur is beter dan helemaal geen buur, vooral als ze van die lekkere taarten bakken en op zaalvoetbalschoenen je het snot voor de ogen lopen. En dan de drijvende krachten achter de vakgroep(en). Anjenet, Liane en Wies. Mijn complimenten dat jullie zo goed uit de voeten kunnen met al die (on)ervaren, gedreven en eigenwijze wetenschappers. Sorry voor alle keren dat mijn verlofkaarten, declaraties en publicatiegegevens met enige vertraging bij jullie binnen kwamen. Eerst met Martijn K, en daarna met B Koning heb ik een super leuke tijd gehad in W205. Een goede afwisseling van hard werken en ontspanning heeft me elke dag met veel plezier naar de UT gebracht. Martijn, hopelijk lukt het me om binnenkort jullie en de kleine eindelijk eens op te zoeken in Zwolle. Bart, in een relatief korte periode heb ik je aardig leren kennen. Ik wens je veel succes met je promotie en ik hoop dat we regelmatig contact zullen houden. Van alle bachelor- en masterstudenten wil ik vooral Birgit, Karel, Mireille en Marlies bedanken voor hun inzet tijdens het afstuderen. Ik hoop dat ik jullie wat heb kunnen leren. Andersom geldt dat in ieder geval wel.

Via deze weg wil ik graag de dwarslaesieverpleegkundigen van het Roessingh bedanken voor hun inzet tijdens onze klinische experimenten. Jullie deskundigheid heeft ervoor gezorgd dat alles prima is verlopen. Ook bedank ik alle patiënten die hebben meegedaan aan ons onderzoek. Jullie deelname is van enorm belang geweest. Eén voor één hebben jullie bijgedragen aan een mooi stukje wetenschap. Hierdoor zijn we weer wat verder in onze voortdurende zoektocht naar het voorkomen van decubitus en andere fysieke ongemakken die ontstaan door langdurig rolstoelgebruik.

Zonder het gehele Dynasit consortium was dit alles niet mogelijk geweest. Elke partner heeft op zijn manier een goede bijdrage geleverd aan het project. Louis en Bert, jullie 
inbreng en expertise heb ik altijd enorm gewaardeerd. Jullie praktische kennis van zaken is onmisbaar gebleken. Naast de UT was ik ook erg vaak te vinden bij RRD. Als gast voelde ik me hier altijd erg op mijn gemak. Iedereen was nooit te beroerd om te helpen tijdens experimenten of om op te komen draven als healthy subject. Hans, Michiel, Maarten, Marc en Govert, jullie goede en veelal kritische feedback (hoe confronterend dat soms ook was) tijdens onze besprekingen en mailwisselingen heb ik enorm gewaardeerd. Leendert, als labbeheerder heb je toch wel het meest met me te stellen gehad. Steeds weer het VR-lab vrijmaken en keer op keer met de VICON camera's slepen. Jouw voorspellende gave heeft ervoor gezorgd dat we na de watersnood het onderzoek meteen konden hervatten. Door op de vooravond de target-PC van de sta-in-de-weg hoog en droog te zetten is ons veel ellende bespaard gebleven. De opstelling staat inmiddels weer bij Demcon in Oldenzaal, weet je zeker dat je hem kunt missen?

De snelcursus bij Demcon was ook zeker niet overbodig. Op allerlei gebieden heb ik erg veel van jullie geleerd. Als projectcoördinator hebben jullie altijd een strakke planning gehandhaafd. Eerst door Hans en Rini, en daarna door Bianca. Ondanks onze vele vergaderingen heeft jullie effectieve manier van werken bijgedragen tot een succesvol project. De professionele wijze waarop jullie werken verdient absoluut een compliment. Ook wil ik hierbij Peter Pieper bedanken voor zijn tomeloze inzet. Je hebt me in sneltreinvaart de mechatronische beginstelen aangeleerd die ik nodig had om de opstelling naar behoren aan te sturen, data te verzamelen en uit te lezen. Als helpdesk op afstand was je, waar dan ook, altijd bij machte om mijn problemen op te lossen. Bedankt voor je enthousiasme en de manier waarop je me in deze periode technisch hebt ondersteund.

Stephen, Maureen and Sharon, thanks for inviting me over en showing me around at Georgia Tech. You guys are an inspiration for this field. Sharon, it is very nice knowing you and thank you for accompanying me during the RESNA 2008. I had a great time! Good luck defending your dissertation and enjoy your future academic career. Keep in touch!

Not last and not least, wil ik hierbij mijn vrienden uit Waarland, Den Haag, Amsterdam en omstreken graag laten weten dat ze me enorm hebben geïnspireerd en ertoe hebben bijgedragen dat ik me steeds opnieuw heb kunnen opladen. Door me uit mijn wetenschappelijke wereldje te trekken en keer op keer te confronteren met zaken buiten mijn onderzoek heb ik alles goed kunnen relativeren. Ik hoop dat onze vriendschap nog lang zal voortduren.

Remi, Evelien en Pauline, met jullie als schoonfamilie heb ik het enorm getroffen. Ze zeggen wel eens, je schoonfamilie krijg je erbij. Dat is inderdaad waar, en daarom ben ik extra blij dat jullie het zijn!

Als onmisbare factor en rode draad door mijn gehele persoonlijke en academische ontwikkeling staat mijn familie nog altijd met stip op een gedeelde eerste plaats. Pap, Mam, Marian, Jos, Harry en Daisy, je familie kies je niet, die heb je gewoon. Bedankt voor jullie steun en toeverlaat tijdens mijn promotie en alles daarnaast. Bewust en onbewust hebben jullie op alle fronten bijgedragen aan dit avontuur. Onmisbaar! Jos, ik vind het erg leuk dat je mijn paranimf wilt zijn. Bedankt hiervoor! 
Lieve, lieve Ilse. Wat heb ik ontzettend veel aan jou te danken. Je onvoorwaardelijke liefde en steun tijdens deze periode is maar moeilijk te verwoorden. Als het soms tegenzat was je er altijd om me op te beuren, en gaf je me de kracht om keer op keer weer verder te gaan. Onze leuke uitjes en vakanties zijn er het laatste jaar een beetje bij in geschoten, maar dat maak ik allemaal goed. Dat beloof ik je. Een nieuwe periode breekt aan. Ons nieuwe huis, Amsterdam, en alles wat nog komen gaat. Ik heb er zin in! 
Curriculum Vitae 


\section{About the author}

Paul van Geffen was born in 1978 in Waarland, the Netherlands. After receiving his high school degree (VWO diploma) at the GSG in Schagen, he studied Human Movement Technology at The Hague University (BSc 2001). During his bachelor degree, he completed a four months research project under the supervision of Stephen H. Sprigle, PhD, PT, at the Research and Development department of Helen Hayes Hospital in New York City, USA. This project was about the design and clinical validation of a digital goniometer that measures hip angle in impaired sitting of spinal cord injured individuals. Back to the Netherlands, he started his bachelor research internship under the supervision of S. de Groot, PhD, and L.H. van der Woude, $\mathrm{PhD}$, at the VU University of Amsterdam where he studied the mechanical efficiency in manual wheelchair propulsion. For his bachelor thesis, he developed and evaluated a novel ankle-foot orthosis that prevents ankle injury in soccer. Rising interests in rehabilitation and sports medicine took him to the VU University of Amsterdam were he started a master program in Human Movement Science (MSc 2004) and specialised himself further in biomechanics and exercise physiology. For his master thesis, he investigated under the supervision of J.J. de Koning (PhD) and F.J. Hettinga (PhD) the effects of pacing strategy on the occurrence of fatigue in cycling. Since January 2005, he has been working as a PhD student in the field of Biomechanical Engineering at the University of Twente (Enschede, The Netherlands). Supervised by H.F.J.M. Koopman, PhD, and P.H. Veltink, PhD, from the Institute of Biomedical Technology (BMTI, University of Twente), he has been involved in the 'Dynasit' project. In this project a dynamic seating system was developed for wheelchair-users with reduced postural stability. Several seating interventions were investigated that control body posture, enhance functional movement and prevent physical discomfort in prolonged static sitting. His PhD dissertation entitled 'Dynamic Sitting' is the result of his contribution to this project.

\section{International Journal Papers}

P. van Geffen, J. Reenalda, P.H. Veltink and H.F.J.M. Koopman (2009). Decoupled pelvis rotation in sitting: A passive motion technique that regulates buttock load associated with pressure ulcer development. Journal of Biomechanics, accepted for publication

J. Reenalda, P. van Geffen, M. Jannink, M.J. IJzerman and H. Rietman (2009). Healthy sitting behavior: Interface pressure and subcutaneous tissue oxygenation. Journal of rehabilitation research and development, accepted for publication.

P. van Geffen, P.H. Veltink and H.F.J.M. Koopman (2009). Can pelvis angle be monitored from seat support forces in healthy subjects? Journal of Biomechanical Engineering, 131(3), 034502, 5 pages 
P. van Geffen, B.I. Molier, J. Reenalda, P.H. Veltink and H.F.J.M. Koopman (2008). Body segments decoupling in sitting: Control of body posture from automatic chair adjustments, Journal of Biomechanics, 41(16), 3419-3425

P. van Geffen, J. Reenalda, P.H. Veltink and H.F.J.M. Koopman (2008). Effects of sagittal postural adjustments on seat reaction load. Journal of Biomechanics, 41(10), 2237 2245

F.J. Hettinga, J.J. de Koning, F.T. Broersen, P. van Geffen, C. Foster C (2006), Pacing strategy and the occurrence of fatigue in 4000-m cycling time trials. Medicine and Science in Sports and Exercise, 38(8), 1484-91

\section{International Conference Participations}

P. van Geffen, J. Reenalda, P.H. Veltink and H.F.J.M. Koopman (2009). The effects of decoupled pelvis rotation on balance control in sitting. Platform presentation in: $2^{\text {nd }}$ Dutch BME Conference 2009 (Dutch Bio-Medical Engineering Society), Egmond aan Zee, the Netherlands.

P. van Geffen, B.I. Molier, J. Reenalda, P.H. Veltink and H.F.J.M. Koopman (2008). A system that adjusts chair configuration for desired postural change. Poster presentation in: RESNA 2008 (Rehabilitation Engineering Society of North America), Washington DC, USA.

P. van Geffen and J. Reenalda (2008). Dynasit: An assistive seating system that controls sitting posture and regulates body load associated with sitting related mobility problems. Platform presentation in: RESNA 2008 (Rehabilitation Engineering Society of North America), Washington DC, USA (paper-award nomination).

P. van Geffen, J. Reenalda, P.H. Veltink and H.F.J.M. Koopman (2007). Effects of actuated pelvis control on shear and pressure distribution. Platform presentation in: ISB 2007 (International Society of Biomechanics), Taipei, Taiwan.

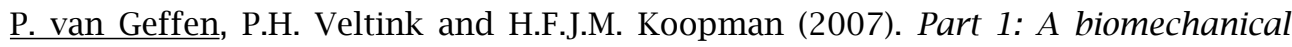
analysis to derive pelvic tilt from seating forces. Platform presentation in: ISS 2007 (International Seating Symposium), Orlando, Florida, USA.

P. van Geffen, P.H. Veltink and H.F.J.M. Koopman (2007). Part 2: An experimental analysis to derive pelvic tilt from seating forces. Platform presentation in: ISS 2007 (International Seating Symposium), Orlando, Florida, USA.

P. van Geffen, H.F.J.M. Koopman and P.H. Veltink (2007). Seating forces to analyse sitting posture and to derive pelvic tilt: Biomechanical and Experimental Analysis Platform presentation in: $1^{\text {st }}$ Dutch BME Conference 2007 (Dutch Bio-Medical Engineering Society), Egmond aan Zee, the Netherlands. 
Publication of this thesis was financially supported by:
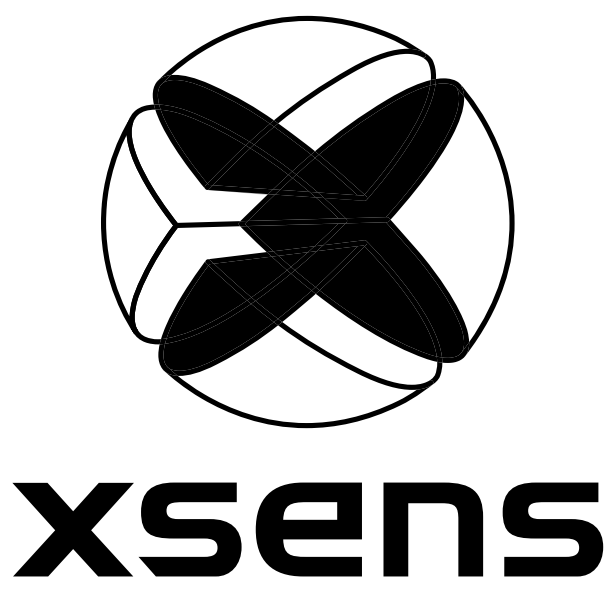

www.xsens.com

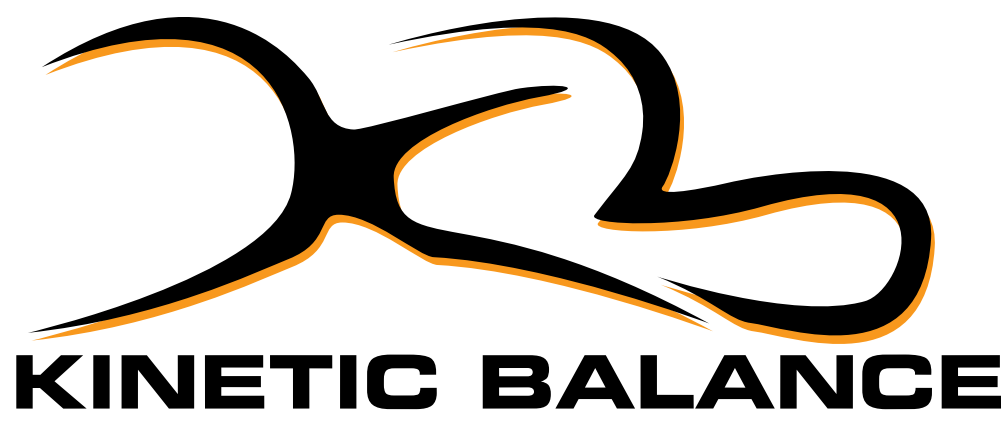

www.positioning-products.nl 
